\title{
Ruthenium-Catalyzed Reductive Cleavage of Unstrained Aryl-Aryl Bonds: Reaction Development and Mechanistic Study
}

\author{
Jun $\mathrm{Zhu}^{1} \dagger$, Peng-hao $\mathrm{Chen}^{2} \dagger$, Gang $\mathrm{Lu}^{3,4 *}$, Peng $\mathrm{Liu}^{3 *}$ and Guangbin Dong ${ }^{1,2 *}$ \\ ${ }^{1}$ Department of Chemistry, University of Chicago, Chicago, Illinois 60637, USA \\ gbdong@uchicago.edu \\ ${ }^{2}$ Department of Chemistry, University of Texas at Austin, Austin, Texas 78712, USA \\ ${ }^{3}$ Department of Chemistry, University of Pittsburgh, Pittsburgh, Pennsylvania 15260, USA \\ pengliu@pitt.edu \\ ${ }^{4}$ School of Chemistry and Chemical Engineering, Shandong University, 250100, Jinan, \\ China
} ganglu@sdu.edu.cn

†These authors contributed equally to this work

\section{Supporting Information}

\section{Contents}

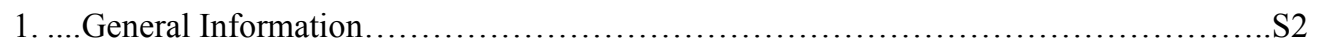

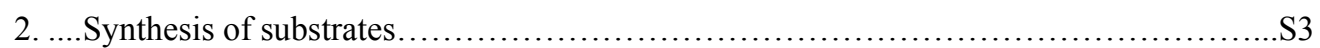

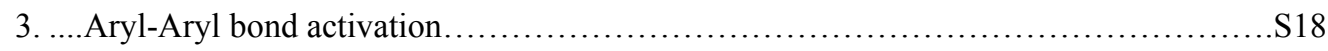

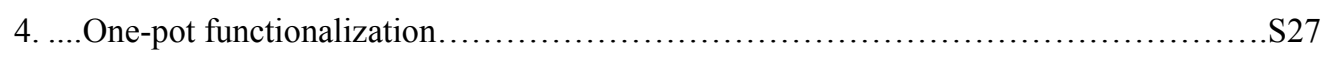

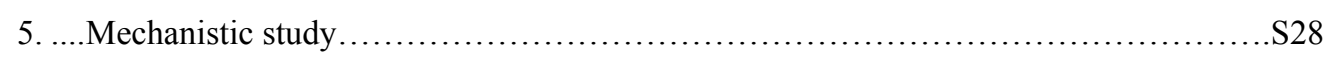

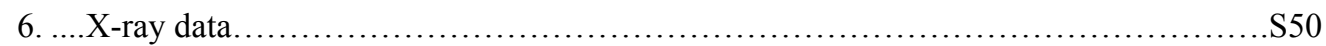

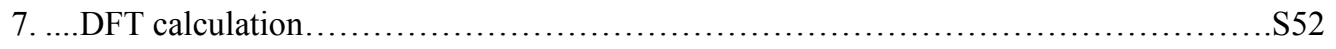

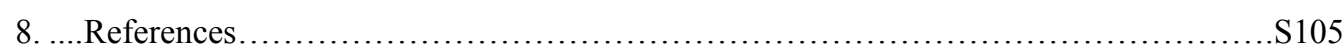

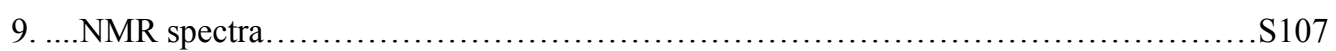




\section{General Information}

All solvents and reagents were obtained from commercial sources and purified according to standard procedures. All glassware was oven dried before use. ${ }^{1}$ H NMR spectra were recorded on a Bruker 500 $\mathrm{MHz}$ or Bruker $400 \mathrm{MHz}$ spectrometer in chloroform- $d$ or benzene- $d_{6}$ or dichloromethane- $d_{2}$ or dimethylsulphoxide- $d_{6}$. All signals are reported in ppm with the internal TMS signal at $0.0 \mathrm{ppm}$ or chloroform signal at $7.26 \mathrm{ppm}$ or dichloromethane at 5.30 or benzene at 7.16 or dimethylsulphoxide at 2.5 as a standard. The data are reported as $(\mathrm{s}=$ singlet, $\mathrm{d}=$ doublet, $\mathrm{t}=$ triplet, $\mathrm{q}=$ quadruplet, $\mathrm{m}=$ multiplet, coupling constant(s) in $\mathrm{Hz}$, integration). ${ }^{13} \mathrm{C}$ NMR spectra were recorded on a Bruker $125 \mathrm{MHz}$ or Bruker $100 \mathrm{MHz}$ spectrometer in chloroform- $d$ or benzene- $d_{6}$ or dichloromethane- $d_{2}$ or or dimethylsulphoxide- $d_{6}$. All signals are reported in ppm with chloroform signal at $77.00 \mathrm{ppm}$ or dichloromethane at 53.84 or benzene at 128.06 or or dimethylsulphoxide at 39.52 as a standard. ${ }^{31} \mathrm{P}$ NMR spectra were recorded on a Bruker $200 \mathrm{MHz}$ spectrometer in chloroform- $d$ or or benzene- $d_{6}$ or dichloromethane- $d_{2}$. IR spectra were recorded on a Nicolet 380 FTIR spectrometer; frequencies are given in reciprocal centimeters $\left(\mathrm{cm}^{-1}\right)$ and only selected absorbance is reported. High-resolution mass spectra (HRMS) was obtained on an Agilent 6224 TOF LC/MS mass spectrometer and are reported as m/z. All pressures are read from the gauge of the Q-tube. 


\section{Synthesis of substrates}

General procedure A: Palladium-catalyzed oxidative dimerization of 2-arylpyridines ${ }^{1}$.

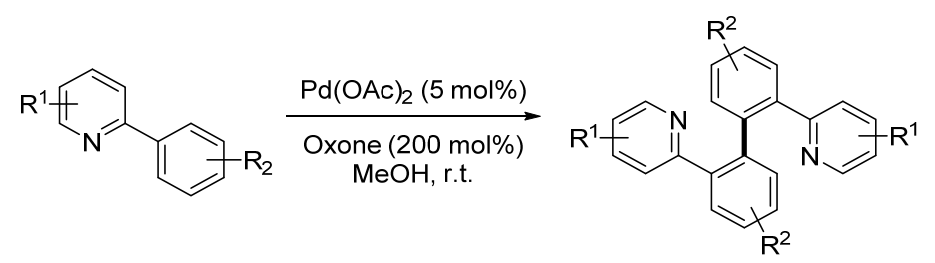

To a flame-dried flask charged with a stir bar, $\mathrm{Pd}(\mathrm{OAc})_{2}(5 \mathrm{~mol} \%)$, Oxone (200 mol\%) and $\mathrm{CH}_{3} \mathrm{OH}(5$ $\mathrm{mL} / 1 \mathrm{mmol}$ ) were added followed by addition of 2-arylpyridines (1.0 equiv.). The reaction mixture was stirred at room temperature under air and was monitored by TLC. Upon completion of the reaction, the reaction mixture was diluted by ethyl acetate and washed with saturated $\mathrm{Na}_{2} \mathrm{SO}_{3}$. The aqueous phase was extracted with ethyl acetate for 3 times. The combined organic layer was washed by brine and dried over $\mathrm{Na}_{2} \mathrm{SO}_{4}$. It was then concentrated under reduced pressure and purified by column chromatography on silica gel to afford the substrates.

General procedure B: Ruthenium-catalyzed oxidative dimerization of 2-arylpyridines ${ }^{2}$.

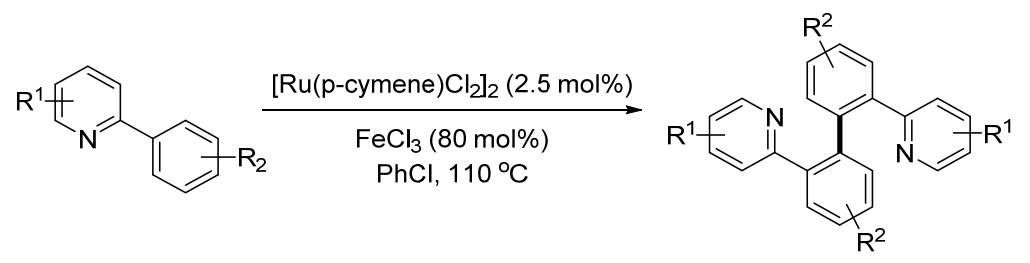

To flame-dried flask charged with a stir bar, $\left[\mathrm{Ru}(\mathrm{p} \text {-cymene }) \mathrm{Cl}_{2}\right]_{2}(2.5 \mathrm{~mol} \%), 2$-arylpyridines (1.0 equiv.). $\mathrm{FeCl}_{3}(80 \mathrm{~mol} \%)$ were added in glovebox. The vail was transferred out of the glovebox, chlorobenzene $(1.6 \mathrm{~mL} / \mathrm{mmol})$ was added in air. The vial was then sealed and the resulting mixture was stirred at $110{ }^{\circ} \mathrm{C}$ for $24 \mathrm{~h}$. After cooling to room temperature, the reaction mixture was poured into $50 \mathrm{~mL} 10 \% \mathrm{NaOH}$ aqueous solution and extracted with ethyl acetate for three times. The combined organic layer was dried over $\mathrm{Na}_{2} \mathrm{SO}_{4}$, concentrated under reduced pressure and purified by column chromatography on silica gel to afford the substrates.

General procedure C: Nickle-mediated dimerization of arylhalide derivatives ${ }^{3}$.

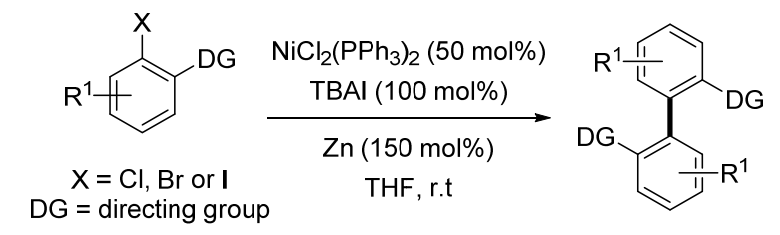

To a flame-dried flask was charged with $\mathrm{NiCl}_{2}\left(\mathrm{PPh}_{3}\right)_{2}(50 \mathrm{~mol} \%)$, TBAI (100 mol\%) and $\mathrm{Zn}(150 \mathrm{~mol} \%)$ and sealed with rubber adaptor. The flask was put under vacuum and backfilled with N2 for three times followed by addition of freshly distilled THF $(1.5 \mathrm{~mL} / \mathrm{mmol})$. The green mixture was stirred at room temperature until a deep red system was afforded. Arylhalide (1.0 equiv.) in freshly distilled THF (0.5 
$\mathrm{ml} / \mathrm{mmol}$ ) was then added to the red solution dropwise at room temperature. After addition, the reaction mixture was stirred at room temperature overnight. The reaction mixture was filtrated through Celite and washed with ethyl acetate. The combined ethyl acetate was washed with water and brine, dried over $\mathrm{Na}_{2} \mathrm{SO}_{4}$, concentrated under reduced pressure and purified by column chromatography on silica gel to afford the dimer.

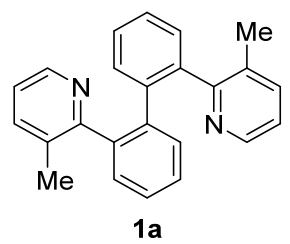

3-methyl-2-phenylpyridine $(337.4 \mathrm{mg}, 2.0 \mathrm{mmol})$ was subjected to general procedure $\mathbf{A}$ to afford 236.1 mg 1a with $69 \%$ yield as a white solid. m.p. $180-182{ }^{\circ} \mathrm{C} .{ }^{1} \mathbf{H}$ NMR $\left(500 \mathrm{MHz}, \mathrm{CDCl}_{3}, 328 \mathrm{~K}\right) \delta 8.27-8.16$ (m, 2H), $7.40-7.16(\mathrm{~m}, 10 \mathrm{H}), 7.03(\mathrm{dd}, J=7.6,4.7 \mathrm{~Hz}, 2 \mathrm{H}), 1.90(\mathrm{~s}, 6 \mathrm{H}) .{ }^{13} \mathbf{C} \mathbf{~ N M R}\left(126 \mathrm{MHz}, \mathrm{CDCl}_{3}\right.$, 328K) $\delta 158.9,146.2,140.2,139.8,137.6,131.9,131.7,130.3,127.3,126.6,121.6,19.4$. HRMS calcd. for $\mathrm{C}_{24} \mathrm{H}_{21} \mathrm{~N}_{2}{ }^{+}[\mathrm{M}+\mathrm{H}]^{+}: 337.1699$, found: 337.1707. IR $\left(\mathrm{cm}^{-1}\right)$ 3054, 1583, 1567, 1435, 1418, 1383, 1119 , 1022.

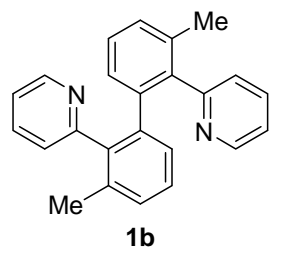

2-(o-tolyl)pyridine (3.6 g, $21.3 \mathrm{mmol}$ ) was subjected to general procedure $\mathbf{A}$ to afford $1.6 \mathrm{~g} \mathbf{1 b}$ with $44 \%$ yield as a white solid. m.p. $157-158{ }^{\circ} \mathrm{C} .{ }^{1} \mathbf{H}$ NMR $\left(400 \mathrm{MHz}, \mathrm{CDCl}_{3}\right) \delta 8.56(\mathrm{~d}, J=4.6 \mathrm{~Hz}, 2 \mathrm{H}), 7.53(\mathrm{t}, J$ $=7.7 \mathrm{~Hz}, 2 \mathrm{H}), 7.31(\mathrm{~d}, J=7.4 \mathrm{~Hz}, 2 \mathrm{H}), 7.10-7.00(\mathrm{~m}, 4 \mathrm{H}), 6.96-6.88(\mathrm{~m}, 2 \mathrm{H}), 6.77(\mathrm{~d}, J=7.4 \mathrm{~Hz}, 2 \mathrm{H})$, $2.10(\mathrm{~s}, 6 \mathrm{H}) .{ }^{13} \mathrm{C}$ NMR $\left(100 \mathrm{MHz}, \mathrm{CDCl}_{3}\right) \delta 159.5,148.7,140.2,139.8,135.9,135.4,128.8,128.5,126.6$, 125.6, 121.1, 20.5. Analytic data match the literature ${ }^{4}$.

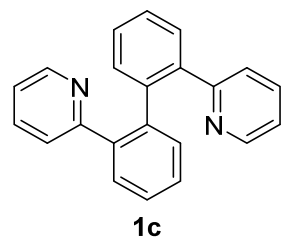

2-phenylpyridine $(500 \mathrm{mg}, 3.2 \mathrm{mmol})$ was subjected to general procedure $\mathbf{A}$ to afford $183.8 \mathrm{mg} \mathbf{1 c}$ with $37 \%$ yield as a white solid. m.p. $134-135{ }^{\circ} \mathrm{C} .{ }^{1} \mathbf{H}$ NMR $\left(500 \mathrm{MHz}, \mathrm{CDCl}_{3}\right) \delta 8.33-8.32(\mathrm{~m}, 2 \mathrm{H}), 7.55-$ $7.53(\mathrm{~m}, 2 \mathrm{H}), 7.44-7.36(\mathrm{~m}, 6 \mathrm{H}), 7.34-7.28(\mathrm{~m}, 2 \mathrm{H}), 7.02-6.99(\mathrm{~m}, 2 \mathrm{H}), 6.76(\mathrm{dd}, J=8.0,1.0 \mathrm{~Hz}, 2 \mathrm{H})$. ${ }^{13}$ C NMR $\left(101 \mathrm{MHz}, \mathrm{CDCl}_{3}\right) \delta 157.9,148.9,139.80,139.76,135.1,131.2,129.9,128.5,127.7,124.3$, 121.1. HRMS calcd. for $\mathrm{C}_{22} \mathrm{H}_{17} \mathrm{~N}_{2}^{+}[\mathrm{M}+\mathrm{H}]^{+}: 309.1386$, found: 309.1394. IR $\left(\mathrm{cm}^{-1}\right) 3054,2360,2341,1585$, $1461,1422,1150,1023$. 


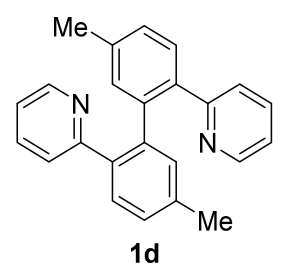

2-(p-tolyl)pyridine $(84.6 \mathrm{mg}, 0.5 \mathrm{mmol})$ was subjected to general procedure $\mathbf{A}$ to afford $35.2 \mathrm{mg} \mathbf{1 d}$ with $42 \%$ yield as a white solid. m.p. $175-177{ }^{\circ} \mathrm{C} .{ }^{1} \mathbf{H}$ NMR $\left(500 \mathrm{MHz}, \mathrm{CDCl}_{3}\right) \delta 8.28-8.27(\mathrm{~m}, 2 \mathrm{H}), 7.41(\mathrm{~d}$, $J=8.0 \mathrm{~Hz}, 2 \mathrm{H}), 7.31-7.26(\mathrm{~m}, 4 \mathrm{H}), 7.21(\mathrm{dd}, J=8.0,1.5 \mathrm{~Hz}, 3 \mathrm{H}), 6.98-6.95(\mathrm{~m}, 2 \mathrm{H}), 6.68(\mathrm{~d}, J=8.0$ $\mathrm{Hz}, 2 \mathrm{H}), 2.43$ (s, 6H). ${ }^{13} \mathbf{C}$ NMR $\left(126 \mathrm{MHz}, \mathrm{CDCl}_{3}\right) \delta 158.0,148.8,139.8,138.3,137.2,135.0,131.9$, 129.9, 128.4, 124.3, 120.8, 21.2. HRMS (m/z): $[\mathrm{M}+\mathrm{H}]^{+}$calcd for $\mathrm{C}_{24} \mathrm{H}_{21} \mathrm{~N}_{2}{ }^{+}, 337.1699$; found, 337.1706. IR $\left(\mathrm{cm}^{-1}\right) 3047,2920,1608,1586,1570,1463,1429,829$.

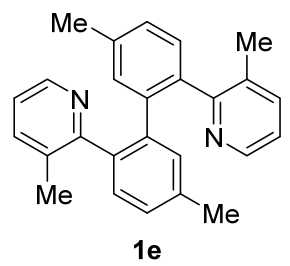

3-methyl-2-(p-tolyl)pyridine $(500 \mathrm{mg}, 2.7 \mathrm{mmol})$ was subjected to general procedure A to afford 356.9 mg 1e with $72 \%$ yield as a white solid. m.p. $147-148{ }^{\circ} \mathrm{C} .{ }^{1} \mathbf{H}$ NMR $\left(500 \mathrm{MHz}, \mathrm{CDCl}_{3}, 328 \mathrm{~K}\right) \delta 8.17$ (d, $J$ $=4.0 \mathrm{~Hz}, 2 \mathrm{H}), 7.31(\mathrm{~d}, J=7.0 \mathrm{~Hz}, 2 \mathrm{H}), 7.13(\mathrm{~s}, 2 \mathrm{H}), 7.08(\mathrm{~s}, 4 \mathrm{H}), 6.98(\mathrm{dd}, J=7.5,4.5 \mathrm{~Hz}, 2 \mathrm{H}), 2.30(\mathrm{~s}$, 6H), 1.83 (s, 6H). ${ }^{13} \mathrm{C}$ NMR (126 MHz, $\left.\mathrm{CDCl}_{3}, 328 \mathrm{~K}\right) \delta 159.0,146.1,140.2,137.4,136.9,136.9,132.6$, 131.7, 130.1, 127.3, 121.4, 21.0, 19.4. HRMS (m/z): $[\mathrm{M}+\mathrm{H}]^{+}$calcd for $\mathrm{C}_{26} \mathrm{H}_{25} \mathrm{~N}_{2}{ }^{+}, 365.2012$; found, 365.2021. IR ( $\left.\mathrm{cm}^{-1}\right)$ 3045, 2952, 2921, 2863, 1608, 1583, 1572, 1443, 1424, 1382, 1117, 1068, 1022.

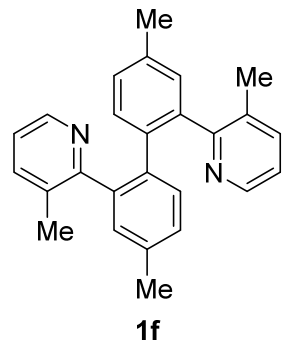

3-methyl-2-(m-tolyl)pyridine $(4.0 \mathrm{~g}, 21.8 \mathrm{mmol})$ was subjected to general procedure B to afford $510.0 \mathrm{mg}$ 1f with $13 \%$ yield as a white solid. m.p. $220-221{ }^{\circ} \mathrm{C} .{ }^{1} \mathbf{H}$ NMR $\left(500 \mathrm{MHz}, \mathrm{CDCl}_{2} \mathrm{CDCl}_{2}, 343 \mathrm{~K}\right) \delta 8.27-$ $8.26(\mathrm{~m}, 2 \mathrm{H}), 7.39-7.37(\mathrm{~m}, 2 \mathrm{H}), 7.10$ (d, J=7.0 Hz, 2H), $7.05-7.00(\mathrm{~m}, 6 \mathrm{H}), 2.34(\mathrm{~s}, 6 \mathrm{H}), 1.99(\mathrm{~s}, 6 \mathrm{H})$. ${ }^{13} \mathbf{C}$ NMR $\left(126 \mathrm{MHz}, \mathrm{CDCl}_{2} \mathrm{CDCl}_{2}, 343 \mathrm{~K}\right) \delta$ 159.5, 146.0, 139.7, 137.4, 137.1, 135.6, 131.9, 130.7, 127.9, 121.5, 21.0, 19.6. HRMS APCI (m/z): $[\mathrm{M}+\mathrm{H}]^{+}$calcd for $\mathrm{C}_{26} \mathrm{H}_{25} \mathrm{~N}_{2}{ }^{+}, 365.2012$; found, 365.2024. IR $\left(\mathrm{cm}^{-1}\right)$ 3046, 3017, 2977, 2952, 2921, 2865, 1584, 1568, 1478, 1458, 1443, 1382, 1266, 1179, 1116. 


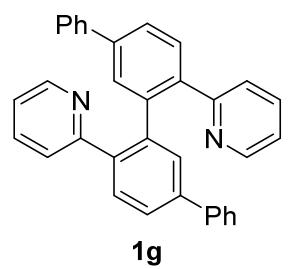

2-([1,1'-biphenyl]-4-yl)pyridine $(0.6 \mathrm{~g}, 2.6 \mathrm{mmol})$ was subjected to general procedure $\mathbf{A}$ to afford 388.9 mg 1 g with 65\% yield as a white solid. m.p. $212-213{ }^{\circ} \mathrm{C} .{ }^{1} \mathbf{H}$ NMR $\left(400 \mathrm{MHz}, \mathrm{CDCl}_{3}\right) \delta 8.38-8.36(\mathrm{~m}$, 2H), $7.75-7.74(\mathrm{~m}, 2 \mathrm{H}), 7.71-7.65(\mathrm{~m}, 8 \mathrm{H}), 7.45-7.44(\mathrm{~m}, 4 \mathrm{H}), 7.39-7.35(\mathrm{~m}, 4 \mathrm{H}), 7.06-7.03$ (m, 2H), $6.90-6.87(\mathrm{~m}, 2 \mathrm{H}) .{ }^{13} \mathrm{C}$ NMR $\left(101 \mathrm{MHz}, \mathrm{CDCl}_{3}\right) \delta 157.6,149.1,141.2,140.2,138.9,135.3,130.7$, 129.9, 128.8, 127.6, 127.1, 126.4, 124.5, 121.3. HRMS ESI (m/z): $[\mathrm{M}+\mathrm{H}]^{+}$calcd for $\mathrm{C}_{34} \mathrm{H}_{25} \mathrm{~N}_{2}{ }^{+}, 461.2012$; found, 461.2021. IR $\left(\mathrm{cm}^{-1}\right)$ 1634, 1584, 1567, 1461, 1431, 1384, 1157.

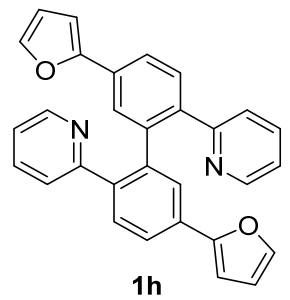

2-(4-(furan-2-yl)phenyl)pyridine $(1.5 \mathrm{~g}, 6.8 \mathrm{mmol})$ was subjected to general procedure A to afford 377.0 mg $\mathbf{1 h}$ with $25 \%$ yield as a pale yellow solid. m.p. $222-223{ }^{\circ} \mathrm{C} .{ }^{\mathbf{1}} \mathbf{H} \mathbf{N M R}\left(500 \mathrm{MHz}, \mathrm{CDCl}_{3}\right) \delta 8.30-8.08$ (m, 2H), $7.88(\mathrm{~d}, J=2.0 \mathrm{~Hz}, 2 \mathrm{H}), 7.77(\mathrm{dd}, J=8.5,2.0 \mathrm{~Hz}, 2 \mathrm{H}), 7.60(\mathrm{~d}, J=8.0 \mathrm{~Hz}, 2 \mathrm{H}), 7.52-7.50(\mathrm{~m}$, 2H), $7.33-7.30(\mathrm{~m}, 2 \mathrm{H}), 7.01-7.00(\mathrm{~m}, 2 \mathrm{H}), 6.77-6.75(\mathrm{~m}, 4 \mathrm{H}), 6.55(\mathrm{dd}, J=8.5,2.4 \mathrm{~Hz}, 2 \mathrm{H}) .{ }^{13} \mathbf{C}$ NMR $\left(126 \mathrm{MHz}, \mathrm{CDCl}_{3}\right) \delta 157.3,153.4,148.9,142.4,140.0,138.8,135.2,131.1,130.5,126.3,124.3$, 123.2, 121.2, 111.8, 105.9. HRMS APCI $(\mathrm{m} / \mathrm{z})$ : $[\mathrm{M}+\mathrm{H}]^{+}$calcd for $\mathrm{C}_{30} \mathrm{H}_{20} \mathrm{~N}_{2} \mathrm{O}_{2}^{+}, 441.1598$; found, 441.1604. IR ( $\left.\mathrm{cm}^{-1}\right) 3121,3101,1709,1607,1585,1567,1474,1425,1394,1369,1287,1214,1158,1151$, 1094, 1065, 1024.

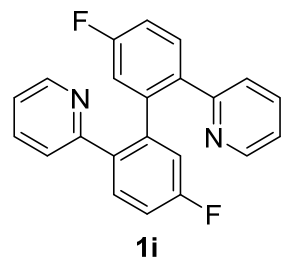

2-(4-fluorophenyl)pyridine $(173.2 \mathrm{mg}, 1.0 \mathrm{mmol})$ was subjected to general procedure B to afford $94.6 \mathrm{mg}$ 1i with 55\% yield as a white solid. m.p. $118-119{ }^{\circ} \mathrm{C} .{ }^{1} \mathbf{H}$ NMR $\left(400 \mathrm{MHz}, \mathrm{CDCl}_{3}\right) \delta 8.32-8.30(\mathrm{~m}, 2 \mathrm{H})$, $7.52-7.49(\mathrm{~m}, 2 \mathrm{H}), 7.38-7.33(\mathrm{~m}, 2 \mathrm{H}), 7.13-7.07(\mathrm{~m}, 4 \mathrm{H}), 7.05-7.00(\mathrm{~m}, 2 \mathrm{H}), 6.78-6.75(\mathrm{~m}, 2 \mathrm{H})$. ${ }^{13}$ C NMR $\left(126 \mathrm{MHz} \mathrm{CDCl}_{3}\right) \delta 162.6(\mathrm{~d}, J=249.5 \mathrm{~Hz}), 156.8,149.0,140.8(\mathrm{~d}, J=7.7 \mathrm{~Hz}), 136.0(\mathrm{~d}, J=$ $3.1 \mathrm{~Hz}), 135.4,132.0(\mathrm{~d}, J=8.6 \mathrm{~Hz}), 124.1,121.4,117.6(\mathrm{~d}, J=22.1 \mathrm{~Hz}), 115.0(\mathrm{~d}, J=20.9 \mathrm{~Hz}) .{ }^{19} \mathbf{F}$ NMR (470 MHz, $\left.\mathrm{CDCl}_{3}\right) \delta$-113.3. HRMS calcd. for $\mathrm{C}_{22} \mathrm{H}_{15} \mathrm{~F}_{2} \mathrm{~N}_{2}{ }^{+}[\mathrm{M}+\mathrm{H}]^{+}:$345.1198, found: 345.1207. IR $\left(\mathrm{cm}^{-1}\right)$ 3050, 1607, 1585, 1494, 1463, 1427, 1252, 1200, 1166. 


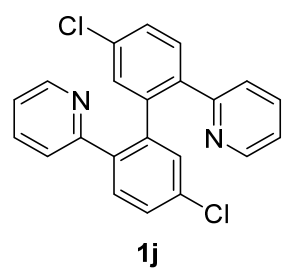

2-(4-chlorophenyl)pyridine $(3.5 \mathrm{~g}, 20.7 \mathrm{mmol})$ was subjected to general procedure B to afford $817.2 \mathrm{mg}$ 1j with $26 \%$ yield as a white solid. ${ }^{1} \mathbf{H}$ NMR $\left(500 \mathrm{MHz}, \mathrm{CDCl}_{3}\right) \delta 8.28(\mathrm{~d}, \mathrm{~J}=4.5 \mathrm{~Hz}, 2 \mathrm{H}), 7.45-7.44(\mathrm{~m}$, 4H), $7.40-7.34(\mathrm{~m}, 4 \mathrm{H}), 7.05-7.02(\mathrm{~m}, 2 \mathrm{H}), 6.71(\mathrm{~d}, J=8.0 \mathrm{~Hz}, 2 \mathrm{H}) .{ }^{13} \mathbf{C} \mathbf{N M R}\left(126 \mathrm{MHz}, \mathrm{CDCl}_{3}\right) \delta$ $156.5,149.1,140.2,138.3,135.5,134.5,131.5,130.7,128.3,124.1,121.5$. Analytic data match the literature ${ }^{5}$.

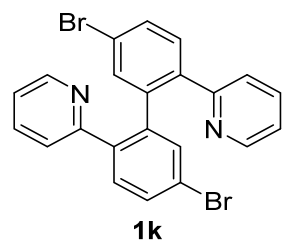

2-(4-bromophenyl)pyridine $(3.5 \mathrm{~g}, 20.7 \mathrm{mmol})$ was subjected to general procedure B to afford $1.5 \mathrm{~g} \mathbf{1 k}$ with $43 \%$ yield as a white solid. m.p. $183-184{ }^{\circ} \mathrm{C} .{ }^{1} \mathbf{H}$ NMR $\left(500 \mathrm{MHz}, \mathrm{CDCl}_{3}\right) \delta 8.28-8.27$ (m, 2H), $7.59(\mathrm{~d}, J=2.0 \mathrm{~Hz}, 2 \mathrm{H}), 7.54(\mathrm{dd}, J=8.5,2.0 \mathrm{~Hz}, 2 \mathrm{H}), 7.37-7.33(\mathrm{~m}, 4 \mathrm{H}), 7.04-7.01(\mathrm{~m}, 2 \mathrm{H}), 6.69(\mathrm{~d}$, $J=8.0 \mathrm{~Hz}, 2 \mathrm{H}) .{ }^{13} \mathbf{C}$ NMR $\left(126 \mathrm{MHz}, \mathrm{CDCl}_{3}\right) \delta 156.4,149.1,140.3,138.7,135.4,133.6,131.6,131.2$, 124.0, 122.7, 121.5. HRMS APCI (m/z): $[\mathrm{M}+\mathrm{H}]^{+}$calcd for $\mathrm{C}_{22} \mathrm{H}_{15} \mathrm{Br}_{2} \mathrm{~N}_{2}^{+}$, 464.9597; found, 464.9604. IR $\left(\mathrm{cm}^{-1}\right)$ 3128, 3043, 3009, 1586, 1565, 1457, 1425, 1402, 1093, 1084, 1016.

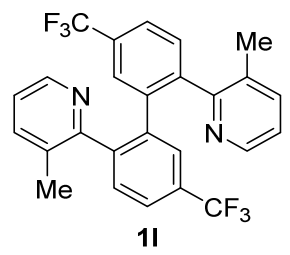

3-methyl-2-(4-(trifluoromethyl)phenyl)pyridine (4.2 g, $17.7 \mathrm{mmol})$ was subjected to general procedure A to afford $909.7 \mathrm{mg} \mathbf{1 l}$ with $22 \%$ yield as a white solid. m.p. $183-185{ }^{\circ} \mathrm{C} .{ }^{1} \mathbf{H}$ NMR (500 MHz, $\left.\mathrm{CDCl}_{2} \mathrm{CDCl}_{2}, 343 \mathrm{~K}\right) \delta 8.36-8.27(\mathrm{~m}, 2 \mathrm{H}), 7.55(\mathrm{dd}, J=8.5,1.5 \mathrm{~Hz}, 2 \mathrm{H}), 7.51(\mathrm{~s}, 2 \mathrm{H}), 7.48(\mathrm{dd}, J=7.0$, $0.5 \mathrm{~Hz}, 2 \mathrm{H}), 7.41(\mathrm{~d}, J=8.0 \mathrm{~Hz}, 2 \mathrm{H}), 7.11(\mathrm{dd}, J=7.5,4.5 \mathrm{~Hz}, 2 \mathrm{H}), 2.08(\mathrm{~s}, 6 \mathrm{H}) .{ }^{13} \mathbf{C} \mathbf{N M R}(126 \mathrm{MHz}$, $\left.\mathrm{CDCl}_{2} \mathrm{CDCl}_{2}, 343 \mathrm{~K}\right) \delta 157.2,146.3,143.5,139.2,137.9,132.0,130.4,129.2$ (q, $\left.J=32.5 \mathrm{~Hz}\right), 128.5(\mathrm{~d}, J$ $=3.4 \mathrm{~Hz}), 124.0(\mathrm{q}, J=265.3 \mathrm{~Hz}), 123.6(\mathrm{~d}, J=3.4 \mathrm{~Hz}), 122.5,19.2 .{ }^{19} \mathbf{F} \mathbf{~ N M R}\left(470 \mathrm{MHz}, \mathrm{CDCl}_{2} \mathrm{CDCl}_{2}\right.$, 343K) $\delta$-61.1. HRMS APCI (m/z): $[\mathrm{M}+\mathrm{H}]^{+}$calcd for $\mathrm{C}_{26} \mathrm{H}_{19} \mathrm{~F}_{6} \mathrm{~N}_{2}{ }^{+}, 473.1447$; found, 473.1458. IR $\left(\mathrm{cm}^{-1}\right)$ $3055,3025,2975,2935,1615,1581,1569,1491,1463,1448,1437,1426,1388,1327,1301,1278,1249$, $1228,1172,1130$. 


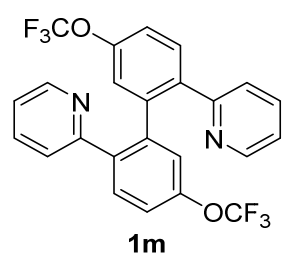

2-(4-(trifluoromethoxy)phenyl)pyridine $(4.0 \mathrm{~g}, 16.7 \mathrm{mmol})$ was subjected to general procedure A to afford $1.2 \mathrm{~g} \mathbf{1 m}$ with $30 \%$ yield as a pale yellow oil. ${ }^{1} \mathbf{H} \mathbf{N M R}\left(500 \mathrm{MHz}, \mathrm{CDCl}_{3}\right) \delta 8.41(\mathrm{~d}, J=5.0 \mathrm{~Hz}$, 2H), $7.62(\mathrm{~d}, J=8.5 \mathrm{~Hz}, 2 \mathrm{H}), 7.46-7.42(\mathrm{~m}, 2 \mathrm{H}), 7.29-7.27(\mathrm{~m}, 2 \mathrm{H}), 7.13-7.09(\mathrm{~m}, 4 \mathrm{H}), 6.95(\mathrm{~d}, J=$ $8.0 \mathrm{~Hz}, 2 \mathrm{H}) .{ }^{13} \mathrm{C}$ NMR $\left(101 \mathrm{MHz}, \mathrm{CDCl}_{3}\right) \delta$ 156.7, 149.3, 148.9 (q, $\left.J=1.9 \mathrm{~Hz}\right), 140.4,138.6,135.6$, $131.7,124.2,123.3(\mathrm{~d}, J=0.8 \mathrm{~Hz}), 121.7,120.35(\mathrm{~d}, J=0.7 \mathrm{~Hz}), 120.31(\mathrm{q}, J=258.8 \mathrm{~Hz}){ }^{\mathbf{1 9}} \mathbf{F} \mathbf{N M R}(470$ MHz, $\left.\mathrm{CDCl}_{3}\right) \delta$-57.9. HRMS-ESI (m/z): $[\mathrm{M}+\mathrm{H}]^{+}$calcd for $\mathrm{C}_{24} \mathrm{H}_{15} \mathrm{~F}_{6} \mathrm{~N}_{2} \mathrm{O}_{2}{ }^{+}, 477.1032$; found, 477.1036 . IR $\left(\mathrm{cm}^{-1}\right) 3051,3010,1606,1587,1574,1566,1490,1464,1429,1393,1256,1217,1167,1094,1067$, $1057,1023$.

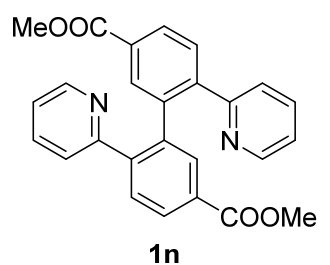

Methyl 4-(pyridin-2-yl)benzoate $(3.5 \mathrm{~g}, 20.7 \mathrm{mmol})$ was subjected to general procedure B to afford 817.2 mg 1n with $26 \%$ yield as a white solid. ${ }^{1} \mathbf{H}$ NMR $\left(500 \mathrm{MHz}, \mathrm{CDCl}_{3}\right) \delta 8.29-8.28(\mathrm{~m}, 2 \mathrm{H}), 8.19$ (d, $J=$ $1.5 \mathrm{~Hz}, 2 \mathrm{H}), 8.08(\mathrm{dd}, J=8.0,2.0 \mathrm{~Hz}, 2 \mathrm{H}), 7.58(\mathrm{t}, J=8.0 \mathrm{~Hz}, 2 \mathrm{H}), 7.34-7.31(\mathrm{~m}, 2 \mathrm{H}), 7.05-7.02(\mathrm{~m}$, 2H), $6.69(\mathrm{~d}, J=8.0 \mathrm{~Hz}, 2 \mathrm{H}), 3.95(\mathrm{~s}, 6 \mathrm{H}) .{ }^{13} \mathbf{C}$ NMR $\left(126 \mathrm{MHz}, \mathrm{CDCl}_{3}\right) \delta 166.6,156.6,149.2,144.0$, 139.2, 135.4, 132.4, 130.4, 130.3, 129.1, 124.3, 121.8, 52.2. Analytic data match the literature ${ }^{6}$.

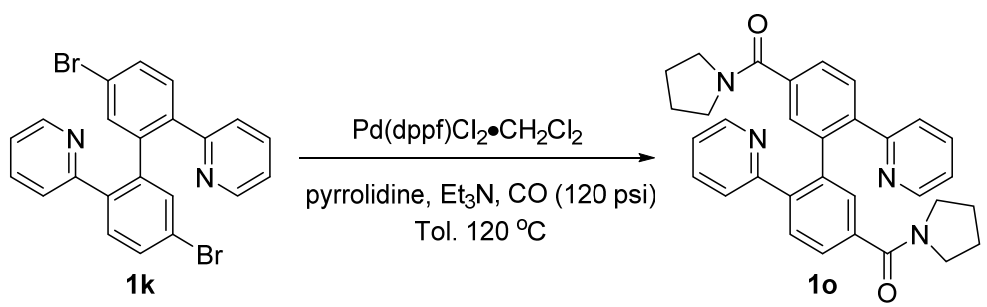

To a Q-tube charged with a stir bar, $1 \mathbf{k}(300 \mathrm{mg}, 0.66 \mathrm{mmol}), \mathrm{Pd}(\mathrm{dppf}) \mathrm{Cl}_{2} \cdot \mathrm{CH}_{2} \mathrm{Cl}_{2}(26.8 \mathrm{mg}, 0.033 \mathrm{~mol})$, pyrrolidine (188 mg, $2.64 \mathrm{mmol}), \mathrm{Et}_{3} \mathrm{~N}(267.1 \mathrm{mg}, 2.64 \mathrm{mmol})$ and toluene $(3.0 \mathrm{~mL})$ were added. The Q-tube was then flushed with CO for three times and charged with 120 psi CO followed heating to $120{ }^{\circ} \mathrm{C}$ for $18 \mathrm{~h}$. Upon completion of the reaction, the reaction mixture was cooled to room temperature, the $\mathrm{CO}$ pressure was released. The solvent was removed under reduced pressure to give a dark oil which was further purified by chromatography on silica gel $\left(\mathrm{CH}_{2} \mathrm{Cl}_{2} / \mathrm{CH}_{3} \mathrm{OH}=4 / 1\right)$ to afford 10 as a brown foam (238.0 mg, 72\%). ${ }^{1} \mathbf{H}$ NMR (500 MHz, $\left.\mathrm{CDCl}_{3}\right) \delta 8.40-8.38(\mathrm{~m}, 2 \mathrm{H}), 7.62(\mathrm{~d}, J=8.0 \mathrm{~Hz}, 2 \mathrm{H}), 7.55$ (dd, $J$ $=7.5,1.5 \mathrm{~Hz}, 2 \mathrm{H}), 7.44-7.40(\mathrm{~m}, 2 \mathrm{H}), 7.35(\mathrm{~d}, J=1.5 \mathrm{~Hz}, 2 \mathrm{H}), 7.08(\mathrm{~d}, J=8.0 \mathrm{~Hz}, 2 \mathrm{H}), 7.06-7.04(\mathrm{~m}$, 
2H), $3.60-3.56(\mathrm{~m}, 4 \mathrm{H}), 3.13-3.07(\mathrm{~m}, 4 \mathrm{H}), 1.93-1.85(\mathrm{~m}, 2 \mathrm{H}), 1.81-1.76(\mathrm{~m}, 4 \mathrm{H}) .{ }^{13} \mathbf{C} \mathbf{N M R}(101$ $\left.\mathrm{MHz}, \mathrm{CDCl}_{3}\right) \delta 168.8,157.6,149.2,141.0,139.3,136.9,135.6,130.3,130.0,126.7,124.6,121.5,49.3$, 46.1, 26.2, 24.3. HRMS APCI (m/z): $[\mathrm{M}+\mathrm{H}]^{+}$calcd for $\mathrm{C}_{32} \mathrm{H}_{31} \mathrm{~N}_{2} \mathrm{O}_{4}{ }^{+}, 503.2442$; found, 503.2457. IR $\left(\mathrm{cm}^{-1}\right)$ 3465, 3048, 2973, 2877, 1621, 1586, 1568, 1550, 1493, 1462, 1425, 1343, 1295, 1266, 1203.

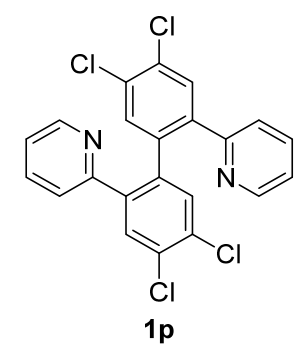

2-(3,4-dichlorophenyl)pyridine $(4.2 \mathrm{~g}, 18.7 \mathrm{mmol})$ was subjected to general procedure $\mathbf{A}$ to afford 383.8 mg 1p with 9\% yield as a pale yellow solid. ${ }^{1} \mathbf{H}$ NMR $\left(500 \mathrm{MHz}, \mathrm{CDCl}_{3}\right) \delta 8.31-8.30(\mathrm{~m}, 2 \mathrm{H}), 7.61(\mathrm{~s}$, 2H), $7.50(\mathrm{~s}, 2 \mathrm{H}), 7.42(\mathrm{td}, J=7.5,1.2 \mathrm{~Hz}, 2 \mathrm{H}), 7.09(\mathrm{dd}, J=7.5,5.0 \mathrm{~Hz}, 2 \mathrm{H}), 6.74(\mathrm{~d}, J=8.0 \mathrm{~Hz}, 2 \mathrm{H})$.

${ }^{13}$ C NMR (101 MHz, $\left.\mathrm{CDCl}_{3}\right) \delta 155.3,149.3,139.5,137.5,135.9,132.8,132.7,132.5,131.9,124.0,122.1$. Analytic data match the literature ${ }^{1}$.

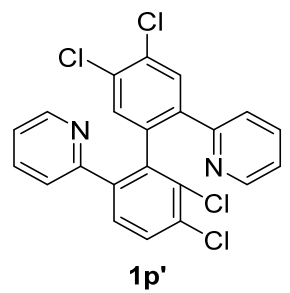

2-(3,4-dichlorophenyl)pyridine $(4.2 \mathrm{~g}, 18.7 \mathrm{mmol})$ was subjected to general procedure A to afford 470.8 mg 1p' with $11 \%$ yield as a pale yellow foam. ${ }^{1} \mathbf{H}$ NMR $\left(500 \mathrm{MHz}, \mathrm{CDCl}_{3}\right) \delta 8.40-8.37(\mathrm{~m}, 2 \mathrm{H}), 7.72(\mathrm{~s}$, 1H), $7.53(\mathrm{~d}, J=8.5,1 \mathrm{H}), 7.49-7.41(\mathrm{~m}, 2 \mathrm{H}), 7.40(\mathrm{~d}, J=8.5 \mathrm{~Hz}, 1 \mathrm{H}), 7.36(\mathrm{~d}, J=6.5 \mathrm{~Hz}, 1 \mathrm{H}), 7.11-$ $7.09(\mathrm{~m}, 2 \mathrm{H}), 7.00(\mathrm{~d}, J=8.0 \mathrm{~Hz}, 1 \mathrm{H}), 6.91(\mathrm{~d}, J=8.0 \mathrm{~Hz}, 1 \mathrm{H}) .{ }^{13} \mathbf{C} \mathbf{N M R}\left(101 \mathrm{MHz}, \mathrm{CDCl}_{3}\right) \delta 156.5$, $155.5,149.2,149.1,140.5,140.0,138.5,136.0,135.9,135.8,133.5,133.3,132.6,132.3,132.2,131.5$, $130.0,129.1,124.2,123.4,122.3,122.1$. Analytic data match the literature ${ }^{1}$.

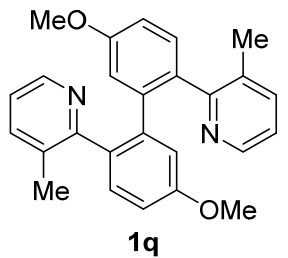

2-(4-methoxyphenyl)-3-methylpyridine $(3.0 \mathrm{~g}, 15.1 \mathrm{mmol})$ was subjected to general procedure $\mathbf{A}$ to afford $636.3 \mathrm{mg} 1 \mathbf{1 q}$ with $22 \%$ yield as a white solid. m.p. $144-146{ }^{\circ} \mathrm{C} .{ }^{1} \mathbf{H} \mathbf{N M R}\left(500 \mathrm{MHz}, \mathrm{CDCl}_{2} \mathrm{CDCl}_{2}, 343 \mathrm{~K}\right)$ $\delta 8.30(\mathrm{dd}, J=4.0,1.0 \mathrm{~Hz}, 2 \mathrm{H}), 7.42$ (dd, $J=7.5 \mathrm{~Hz}, 0.5,2 \mathrm{H}), 7.14$ (d, $J=8.5 \mathrm{~Hz}, 2 \mathrm{H}), 7.05$ (dd, $J=8.0$, $5.0 \mathrm{~Hz}, 2 \mathrm{H}), 6.82(\mathrm{dd}, J=8.5,3.0 \mathrm{~Hz}, 2 \mathrm{H}), 6.75(\mathrm{~d}, J=2.5 \mathrm{~Hz}, 2 \mathrm{H}), 3.64(\mathrm{~s}, 6 \mathrm{H}), 2.07(\mathrm{~s}, 6 \mathrm{H}) .{ }^{13} \mathrm{C}$ NMR $\left(126 \mathrm{MHz}, \mathrm{CDCl}_{2} \mathrm{CDCl}_{2}, 343 \mathrm{~K}\right) \delta 159.3,158.4,146.0,141.1,137.5,132.7,132.3,131.1,121.5,116.5$, 
113.6, 55.4, 19.6. HRMS APCI (m/z): $[\mathrm{M}+\mathrm{H}]^{+}$calcd for $\mathrm{C}_{26} \mathrm{H}_{25} \mathrm{~N}_{2} \mathrm{O}_{2}{ }^{+}, 379.1911$; found, 379.1924. IR $\left(\mathrm{cm}^{-1}\right)$ 3048, 2999, 2967, 2934, 2911, 2834, 1604, 1582, 1564, 1483, 1468, 1451, 1419, 1391, 1300, 1286, $1268,1259,1248,1219,1205,1182,1139$.

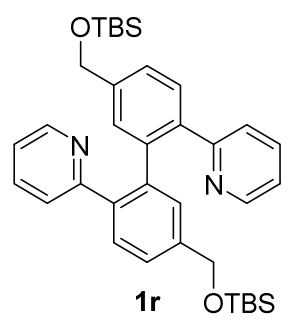

2-(4-(((tert-butyldimethylsilyl)oxy)methyl)phenyl)pyridine $(1.7 \mathrm{~g}, 5.7 \mathrm{mmol})$ was subjected to general procedure A to afford $650.0 \mathrm{mg} \mathbf{1 r}$ with $38 \%$ yield as a colorless oil. ${ }^{1} \mathbf{H}$ NMR $\left(500 \mathrm{MHz}, \mathrm{CDCl}_{3}\right) \delta 8.30$ - $8.28(\mathrm{~m}, 2 \mathrm{H}), 7.50$ (dd, $J=7.0,0.8 \mathrm{~Hz}, 2 \mathrm{H}), 7.41-7.36(\mathrm{~m}, 4 \mathrm{H}), 7.31-7.27(\mathrm{~m}, 2 \mathrm{H}), 7.00-6.97$ (m, $2 \mathrm{H}), 6.72-6.71(\mathrm{~m}, 2 \mathrm{H}), 4.81(\mathrm{ABd}, J=8.0 \mathrm{~Hz}, 4 \mathrm{H}), 0.94(\mathrm{~s}, 18 \mathrm{H}), 0.11(\mathrm{~s}, 12 \mathrm{H}) .{ }^{13} \mathrm{C}$ NMR $(126 \mathrm{MHz}$, $\left.\mathrm{CDCl}_{3}\right) \delta 157.9,148.9,141.9,139.8,138.6,135.1,130.0,128.9,125.6,124.4,121.0,64.8,26.0,18.4,-5.1$. HRMS APCI (m/z): $[\mathrm{M}+\mathrm{H}]^{+}$calcd for $\mathrm{C}_{36} \mathrm{H}_{49} \mathrm{~N}_{2} \mathrm{O}_{2} \mathrm{Si}_{2}{ }^{+}, 597.3327$; found, 597.3340. IR $\left(\mathrm{cm}^{-1}\right) 3049,3006$, 2953, 2927, 2884, 2855, 2803, 2772, 1704, 1608, 1587, 1573, 1470, 1463, 1434, 1402, 1390, 1361, 1294 , 1255, 1203, 1173.

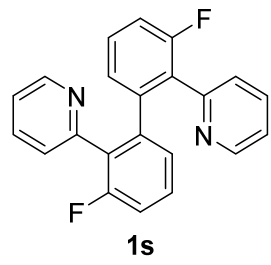

2-(2-fluorophenyl)pyridine $(173.2 \mathrm{mg}, 1.0 \mathrm{mmol})$ was subjected to general procedure $\mathbf{A}$ to afford 128.2 mg 1s with $74 \%$ yield as a white solid. m.p. $125-127{ }^{\circ} \mathrm{C} .{ }^{1} \mathbf{H}$ NMR $\left(500 \mathrm{MHz}, \mathrm{CDCl}_{3}\right) \delta 8.47-8.46(\mathrm{~m}$, 2H), $7.52-7.49(\mathrm{~m}, 2 \mathrm{H}), 7.22(\mathrm{dd}, J=8.0,1.0 \mathrm{~Hz}, 1 \mathrm{H}), 7.18-7.13(\mathrm{~m}, 2 \mathrm{H}), 7.11-7.08(\mathrm{~m}, 2 \mathrm{H}), 7.04-$ $7.01(\mathrm{~m}, 2 \mathrm{H}), 6.88(\mathrm{~d}, J=8.0 \mathrm{~Hz}, 2 \mathrm{H}) .{ }^{13} \mathbf{C} \mathbf{N M R}\left(126 \mathrm{MHz}, \mathrm{CDCl}_{3}\right) \delta 160.0(\mathrm{~d}, J=248.1 \mathrm{~Hz}), 153.4$, 148.9, 141.7, 135.5, 128.8 (d, $J=9.0 \mathrm{~Hz}), 128.0(\mathrm{~d}, J=14.9 \mathrm{~Hz}), 126.9$ (d, $J=3.3 \mathrm{~Hz}), 126.0,121.9$, $114.6(\mathrm{~d}, J=22.4 \mathrm{~Hz}) .{ }^{19} \mathbf{F}$ NMR $\left(470 \mathrm{MHz}, \mathrm{CDCl}_{3}\right) \delta$-116.7. HRMS $(\mathrm{m} / \mathrm{z}):[\mathrm{M}+\mathrm{H}]^{+}$calcd for $\mathrm{C}_{22} \mathrm{H}_{15} \mathrm{~F}_{2} \mathrm{~N}_{2}{ }^{+}$, 345.1198; found, 345.1203. IR ( $\left.\mathrm{cm}^{-1}\right)$ 3048, 1609, 1587, 1571, 1497, 1452, 1424, 1238, 1151, 1126, 1026.

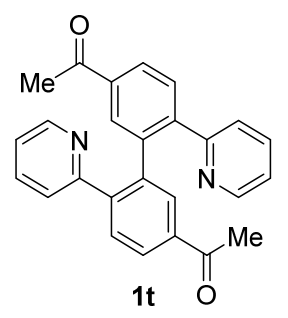

1-(4-(pyridin-2-yl)phenyl)ethan-1-one $(59.2 \mathrm{mg}, 0.3 \mathrm{mmol})$ was subjected to general procedure A to 
afford $28.6 \mathrm{mg} \mathbf{1 t}$ with $49 \%$ yield as a white solid. m.p. $105-107{ }^{\circ} \mathrm{C} .{ }^{1} \mathbf{H}$ NMR $\left(500 \mathrm{MHz}, \mathrm{CDCl}_{3}\right) \delta 8.31-$ $8.29(\mathrm{~m}, 2 \mathrm{H}), 8.00-7.95(\mathrm{~m}, 4 \mathrm{H}), 7.61-7.58(\mathrm{~m}, 2 \mathrm{H}), 7.36-7.32(\mathrm{~m}, 2 \mathrm{H}), 7.05-7.00(\mathrm{~m}, 2 \mathrm{H}), 6.79(\mathrm{~d}$, $J=8.0 \mathrm{~Hz}, 2 \mathrm{H}), 2.57$ (s, 6H). ${ }^{13} \mathbf{C}$ NMR $\left(101 \mathrm{MHz}, \mathrm{CDCl}_{3}\right) \delta 197.4,156.7,149.1,144.0,139.4,136.8$, 135.5, 131.2, 130.4, 127.6, 124.2, 121.8, 26.7. HRMS calcd. for $\mathrm{C}_{26} \mathrm{H}_{21} \mathrm{~N}_{2} \mathrm{O}_{2}{ }^{+}[\mathrm{M}+\mathrm{H}]^{+}: 393.1598$, found: 393.1605. IR $\left(\mathrm{cm}^{-1}\right)$ 1683, 1585, 1464, 1431, 1385, 1357, 1293, 1268, 1021.

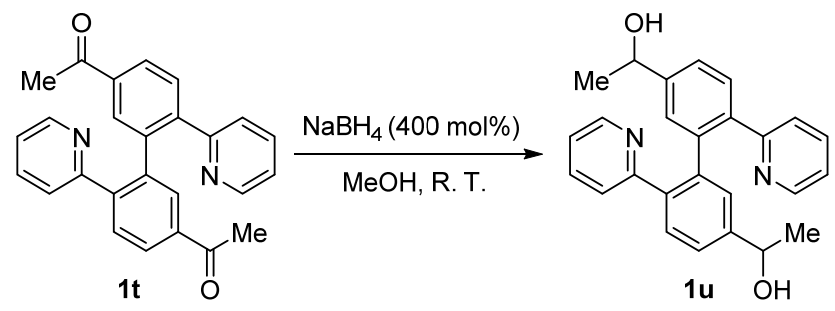

To a round bottom flask with a stir bar was added $\mathbf{1 t}$ (700 mg, $1.78 \mathrm{mmol}, 1$ equiv.) and $\mathrm{NaBH}_{4}(270$ $\mathrm{mg}, 7.12 \mathrm{mmol}, 4$ equiv.) followed by the addition of $\mathrm{MeOH}(15 \mathrm{~mL})$. The reaction mixture was allowed to stir at R. T. and monitored by TLC. Upon reaction completion, saturated ammonium chloride was added to quench the reaction, followed by the addition of $1 \mathrm{M} \mathrm{HCl}$ solution. The reaction mixture was allowed to stir at R. T. for 20 min before trimethylamine was added. The reaction mixture was extracted with ethyl acetate $(2 \times 20 \mathrm{~mL})$, and the combined organic extract was washed with brine, dried over $\mathrm{Na}_{2} \mathrm{SO}_{4}$. After concentrated under reduced pressure, the residue was purified via column chromatography to afford $\mathbf{1 u}$ in full conversion. Right after column, compound 1u was obtained as a mixture of inseparable diastereomers. m. p. $99-100{ }^{\circ} \mathrm{C} .{ }^{1} \mathbf{H}$ NMR $\left(400 \mathrm{MHz}, \mathrm{CDCl}_{3}+\mathrm{D}_{2} \mathrm{O}\right) \delta 8.63-8.44(\mathrm{~m}, 2 \mathrm{H}), 7.54-7.37(\mathrm{~m}, 3.45 \mathrm{H})$, $7.36-7.24(\mathrm{~m}, 2.22 \mathrm{H}), 7.15-7.02(\mathrm{~m}, 2.64 \mathrm{H}), 6.75-6.59(\mathrm{~m}, 2.02 \mathrm{H}), 5.82(\mathrm{~s}, 0.51 \mathrm{H}), 5.05(\mathrm{~s}, 0.50 \mathrm{H})$, $4.92(\mathrm{~s}, 0.85 \mathrm{H}), 4.87-4.76(\mathrm{~m}, 0.61 \mathrm{H}), 4.05-3.91(\mathrm{~m}, 1.35 \mathrm{H}), 0.98-0.79(\mathrm{~m}, 6 \mathrm{H}) .{ }^{13} \mathrm{C}$ NMR $(101 \mathrm{MHz}$, $\left.\mathrm{CDCl}_{3}+\mathrm{D}_{2} \mathrm{O}\right) \delta 159.6,159.0,158.2,148.8,148.5,147.8,147.7,147.6,138.7,138.2,137.5,137.4,137.2$, $135.6,135.3,130.8,130.6,130.3,130.1,129.9,127.3,125.6,125.3,124.8,123.7,123.7,121.5,121.4$, 69.2, 69.1, 68.3, 25.7, 25.6, 25.4. HRMS calcd. for $\mathrm{C}_{26} \mathrm{H}_{25} \mathrm{~N}_{2} \mathrm{O}_{2}{ }^{+}[\mathrm{M}+\mathrm{H}]^{+}$: 397.1911, found: 397.1919. IR $\left(\mathrm{cm}^{-1}\right) 3283,2970,1591,1568,1490,1464,1433,1385,1367,1295,1101,1074,1021,955,905,841,792$, $751,711$.

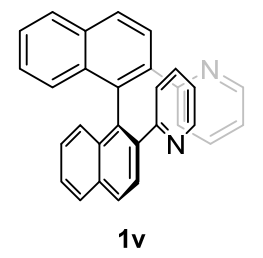

2-(1-chloronaphthalen-2-yl)pyridine (1 g, $4.17 \mathrm{mmol}$ ) was subjected to general procedure $\mathbf{C}$ to afford 612.3 mg $1 \mathbf{v}$ with $72 \%$ yield as a white solid. ${ }^{1} \mathbf{H}$ NMR $\left(400 \mathrm{MHz}, \mathrm{C}_{6} \mathrm{D}_{6}\right) \delta 8.34-8.26(\mathrm{~m}, 2 \mathrm{H}), 8.19-8.09(\mathrm{~m}$, 2H), $7.80(\mathrm{~d}, J=8.5 \mathrm{~Hz}, 2 \mathrm{H}), 7.67(\mathrm{~d}, J=8.1 \mathrm{~Hz}, 2 \mathrm{H}), 7.59$ (dd, $J=8.5,0.5 \mathrm{~Hz}, 2 \mathrm{H}), 7.20-7.09$ (m, 2H), 6.99 (ddd, $J=8.2,6.8,1.2 \mathrm{~Hz}, 2 \mathrm{H}), 6.95$ (dt, $J=8.0,1.0 \mathrm{~Hz}, 2 \mathrm{H}), 6.61$ (td, $J=7.7,1.9 \mathrm{~Hz}, 2 \mathrm{H}), 6.35$ (ddd, $J$ 
$=7.5,4.8,1.1 \mathrm{~Hz}, 2 \mathrm{H}) .{ }^{13} \mathbf{C}$ NMR 159.0, 149.2, 139.6, 135.2, 135.0, 134.0, 133.8, 128.9, 128.5, 128.4, 126.9, 126.4, 124.1, 121.2. The ${ }^{1} \mathrm{H}$ NMR match the literature ${ }^{7}$.

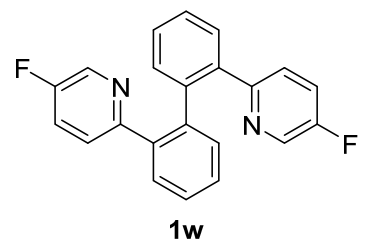

5-fluoro-2-phenylpyridine $(3.0 \mathrm{~g}, 17.3 \mathrm{mmol})$ was subjected to general procedure A to afford $377.0 \mathrm{mg}$ 1w with $13 \%$ yield as a white solid. m.p. $172-173{ }^{\circ} \mathrm{C} .{ }^{1} \mathbf{H}$ NMR $\left(500 \mathrm{MHz}, \mathrm{CDCl}_{3}\right) \delta 8.20(\mathrm{~d}, J=3.0 \mathrm{~Hz}$, 2H), $7.54-7.46(\mathrm{~m}, 2 \mathrm{H}), 7.46-7.39(\mathrm{~m}, 4 \mathrm{H}), 7.38-7.32(\mathrm{~m}, 2 \mathrm{H}), 7.10-6.99(\mathrm{~m}, 2 \mathrm{H}), 6.79(\mathrm{dd}, J=8.5$, $4.0 \mathrm{~Hz}, 1 \mathrm{H}) .{ }^{13} \mathbf{C}$ NMR $\left(101 \mathrm{MHz}, \mathrm{CDCl}_{3}\right) \delta 158.0(\mathrm{~d}, J=257.2 \mathrm{~Hz}), 154.2(\mathrm{~d}, J=4.2 \mathrm{~Hz}), 139.6,138.8$, $137.0(\mathrm{~d}, J=23.1 \mathrm{~Hz}), 131.2,130.0,128.7,127.8,125.0(\mathrm{~d}, J=4.3 \mathrm{~Hz}), 122.2(\mathrm{~d}, J=18.5 \mathrm{~Hz}) .{ }^{19}$ F NMR $\left(470 \mathrm{MHz}, \mathrm{CDCl}_{3}\right.$,) $\delta$-129.8. HRMS APCI $(\mathrm{m} / \mathrm{z})$ : $[\mathrm{M}+\mathrm{H}]^{+}$calcd for $\mathrm{C}_{22} \mathrm{H}_{15} \mathrm{~F}_{2} \mathrm{~N}_{2}{ }^{+}, 345.1198$; found, 345.1212. IR ( $\left.\mathrm{cm}^{-1}\right)$ 3036, 3014, 1588, 1579, 1491, 1473, 1467, 1438, 1414, 1399, 1380, 1274, 1267, 1231, 1222, 1127, 1119.

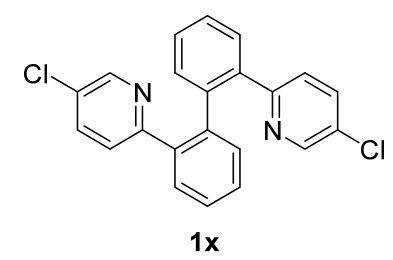

5-chloro-2-phenylpyridine $(2.5 \mathrm{~g}, 13.2 \mathrm{mmol})$ was subjected to general procedure A to afford $516.7 \mathrm{mg}$ $\mathbf{1 x}$ with $21 \%$ yield as a white solid. m.p. $182-183{ }^{\circ} \mathrm{C} .{ }^{1} \mathbf{H}$ NMR $\left(500 \mathrm{MHz}, \mathrm{CDCl}_{3}\right) \delta 8.31(\mathrm{dd}, J=4.0,0.5$ $\mathrm{Hz}, 2 \mathrm{H}), 7.53-7.51(\mathrm{~m}, 2 \mathrm{H}), 7.43-7.40(\mathrm{~m}, 4 \mathrm{H}), 7.36-7.31(\mathrm{~m}, 4 \mathrm{H}), 6.77(\mathrm{dd}, J=8.5,0.5 \mathrm{~Hz}, 2 \mathrm{H}) .{ }^{13} \mathbf{C}$ NMR (101 MHz, $\left.\mathrm{CDCl}_{3}\right) \delta$ 377.0613. 156.2, 147.9, 139.5, 138.6, 135.1, 131.3, 130.0, 129.9, 128.9, 127.9, 124.9. HRMS APCI (m/z): $[\mathrm{M}+\mathrm{H}]^{+}$calcd for $\mathrm{C}_{22} \mathrm{H}_{15} \mathrm{Cl}_{2} \mathrm{~N}_{2}^{+}, 377.0607$; found, 377.0613. IR $\left(\mathrm{cm}^{-1}\right) 3073$, $3031,3001,1573,1552,1455,1435,1405,1372,1300,1222,1139,1114,1069,1009$.

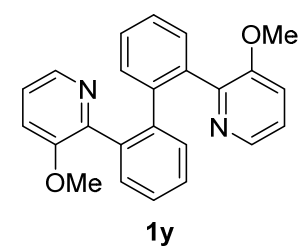

2-methoxy-6-phenylpyridine $(4.0 \mathrm{~g}, 21.6 \mathrm{mmol})$ was subjected to general procedure A to afford $1.85 \mathrm{~g} 1 \mathbf{y}$ with $47 \%$ yield as a white solid. m.p. $204-206{ }^{\circ} \mathrm{C} .{ }^{1} \mathbf{H} \mathbf{N M R}\left(500 \mathrm{MHz}, \mathrm{CDCl}_{3}\right) \delta 7.87(\mathrm{dd}, J=4.5,1.0 \mathrm{~Hz}$, 2H), $7.54-7.49$ (m, 4H), $7.35-7.32(\mathrm{~m}, 4 \mathrm{H}), 7.07$ (dd, $J=8.0,4.5 \mathrm{~Hz}, 2 \mathrm{H}), 6.89$ (dd, $J=8.2,1.0 \mathrm{~Hz}$, 2H), 3.15 (s, 6H). ${ }^{13} \mathbf{C}$ NMR (126 MHz, $\left.\mathrm{CDCl}_{3}\right) \delta 152.3,148.6,142.1,141.2,131.3,130.3,128.0,126.9$, 122.6, 117.3, 54.2. HRMS APCI (m/z): $[\mathrm{M}+\mathrm{H}]^{+}$calcd for $\mathrm{C}_{24} \mathrm{H}_{21} \mathrm{~N}_{2} \mathrm{O}_{2}{ }^{+}, 369.1598$; found, 369.1618. IR $\left(\mathrm{cm}^{-1}\right)$ 3062, 3049, 3027, 2977, 2945, 1580, 1562, 1485, 1465, 1448, 1421, 1279, 1241, 1192, 1130, 1114 , $1072,1052$. 


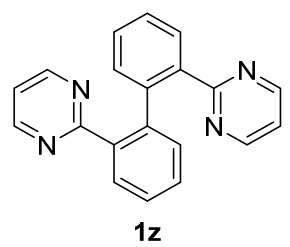

2-(2-chlorophenyl)pyrimidine (330 $\mathrm{mg}, 1.73 \mathrm{mmol}$ ) was subjected to general procedure $\mathbf{C}$ to afford 163.4 mg $1 z$ with $61 \%$ yield as a white solid. m.p. $152-153{ }^{\circ} \mathrm{C} .{ }^{1} \mathbf{H}$ NMR $\left(400 \mathrm{MHz}, \mathrm{CDCl}_{3}\right) \delta 8.42(\mathrm{~d}, J=4.8$ Hz, 4H), $7.64-7.62(\mathrm{~m}, 2 \mathrm{H}), 7.56-7.54(\mathrm{~m}, 4 \mathrm{H}), 7.43-7.39(\mathrm{~m}, 2 \mathrm{H}), 6.99(\mathrm{t}, J=4.8 \mathrm{~Hz}, 2 \mathrm{H}) .{ }^{13} \mathbf{C} \mathbf{N M R}$ $\left(101 \mathrm{MHz}, \mathrm{CDCl}_{3}\right) \delta 166.5,156.5,141.3,137.9,131.2,129.82,129.80,127.2,118.2$. HRMS calcd. for $\mathrm{C}_{20} \mathrm{H}_{15} \mathrm{~N}_{4}^{+}[\mathrm{M}+\mathrm{H}]^{+}:$311.1291, found: 311.1299. IR $\left(\mathrm{cm}^{-1}\right)$ 1567, 1553, 1448, 1412, 820, 754, 731, 644, 636.

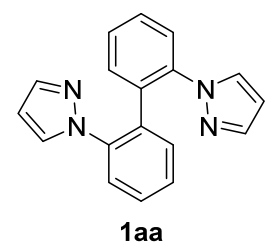

1-(2-bromophenyl)-1H-pyrazole $(5.0 \mathrm{~g}, 22.41 \mathrm{mmol})$ was subjected to general procedure $\mathbf{C}$ to afford 1.31 g 1 aa with $41 \%$ yield as a white solid. m.p. $131-132{ }^{\circ} \mathrm{C} .{ }^{1} \mathbf{H}$ NMR $\left(400 \mathrm{MHz}, \mathrm{CDCl}_{3}\right) \delta 7.51(\mathrm{dd}, J=8.0$, $1.1 \mathrm{~Hz}, 2 \mathrm{H}), 7.49-7.47(\mathrm{~m}, 2 \mathrm{H}), 7.42(\mathrm{td}, J=7.7,1.5 \mathrm{~Hz}, 2 \mathrm{H}), 7.32(\mathrm{td}, J=7.5,1.3 \mathrm{~Hz}, 2 \mathrm{H}), 7.23(\mathrm{~d}, J=$ $2.4 \mathrm{~Hz}, 2 \mathrm{H}), 7.21(\mathrm{dd}, J=7.7,1.4 \mathrm{~Hz}, 2 \mathrm{H}), 6.21-6.15(\mathrm{~m}, 2 \mathrm{H}) .{ }^{13} \mathbf{C}$ NMR $\left(101 \mathrm{MHz}, \mathrm{CDCl}_{3}\right) \delta 140.3$, $138.8,133.3,130.9,130.2,128.8,127.7,125.5,106.4$. Analytic data match the literature ${ }^{8}$.

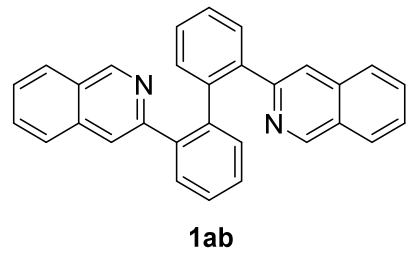

3-phenylisoquinoline $(581.4 \mathrm{mg}, 5.7 \mathrm{mmol})$ was subjected to general procedure A to afford $104.4 \mathrm{mg} \mathbf{1 a b}$ with $18 \%$ yield as a pale yellow solid. m.p. $139-140{ }^{\circ} \mathrm{C} .{ }^{1} \mathbf{H}$ NMR $\left(400 \mathrm{MHz}, \mathrm{CDCl}_{3}\right) \delta 8.79$ (s, 2H), 7.87 - $7.80(\mathrm{~m}, 2 \mathrm{H}), 7.59-7.47(\mathrm{~m}, 8 \mathrm{H}), 7.45-7.36(\mathrm{~m}, 4 \mathrm{H}), 7.33-7.28(\mathrm{~m}, 2 \mathrm{H}), 6.94(\mathrm{~s}, 2 \mathrm{H}) .{ }^{13} \mathbf{C}$ NMR $\left(101 \mathrm{MHz}, \mathrm{CDCl}_{3}\right) \delta 152.1,151.2,140.6,139.7,135.8,131.6,130.1,129.9,128.2,127.4,127.2,126.8$, 126.7, 126.6, 120.5. HRMS calcd. for $\mathrm{C}_{30} \mathrm{H}_{21} \mathrm{~N}_{2}^{+}[\mathrm{M}+\mathrm{H}]^{+}:$409.1699, found: 409.1708. IR (cm $\left.{ }^{-1}\right) 1651$, $1580,1488,1435,1384,1139,1093$. 


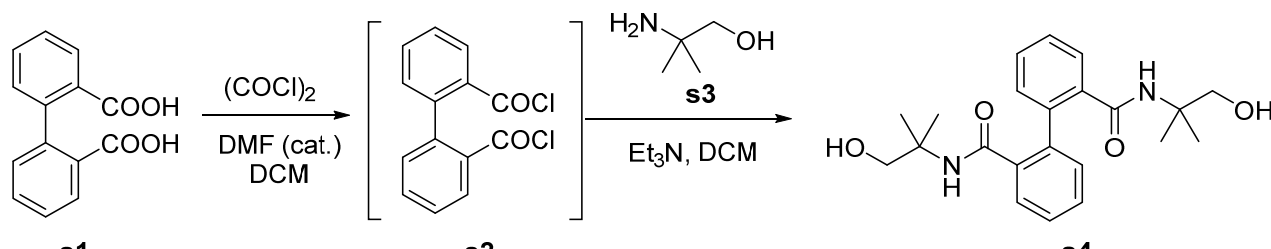

s1

s2

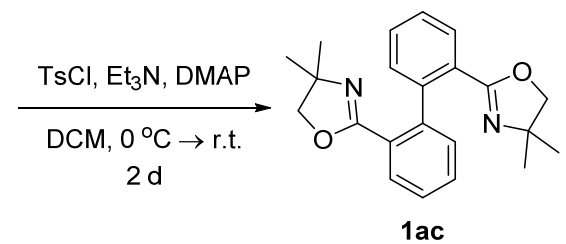

To a suspension of [1,1'-biphenyl]-2,2'-dicarboxylic acid $\mathbf{s 1}(7.3 \mathrm{~g}, 30 \mathrm{mmol})$ in $100 \mathrm{~mL}$ dry dichloromethane in a $200 \mathrm{~mL}$ schlenk flask, 5 drops of dry DMF was added. Oxalyl chloride (15.2 g, 120 mmol) was subsequently added dropwise to the above suspension. The resulting reaction mixture was stirred at room temperature for $6 \mathrm{~h}$. Upon completion of the reaction, the solvent was removed under reduced pressure to afford the acyl chloride as a light yellow solid. The crude acyl chloride was then dissolved in dichloromethane $(100 \mathrm{~mL})$ and slowly added to a stirred solution of 2-amino-2-methylpropan-1-ol $(5.8 \mathrm{~g}, 65 \mathrm{mmol})$ and $\mathrm{Et}_{3} \mathrm{~N}(10.0 \mathrm{~mL})$ in dichloromethane $(100 \mathrm{~mL})$ at $0{ }^{\circ} \mathrm{C}$ over $30 \mathrm{~min}$. The reaction mixture was allowed to warm to room temperature slowly and stirred overnight under nitrogen. Upon completion of the reaction, the reaction mixture was washed by water $(50 \mathrm{~mL} * 3)$ and brine $(50 \mathrm{ml} * 1)$. The organic layer was dried over $\mathrm{Na}_{2} \mathrm{SO}_{4}$, filtered and concentrated under reduced pressure to afford the di-amide $\mathbf{s} 4(11.11 \mathrm{~g})$ as a colorless oil which was used directly in the next step without any purification. The di-amide $\mathbf{s} 4$ was then dissolved in $150 \mathrm{~mL}$ of dry dichloromethane, before DMAP (353 mg, $2.89 \mathrm{mmol})$ and $\mathrm{Et}_{3} \mathrm{~N}(12.9 \mathrm{~g}, 127 \mathrm{mmol})$ were added. The resulting solution was cooled to $0{ }^{\circ} \mathrm{C}$. A solution of 4-toluenesulfonyl chloride $(11.0 \mathrm{~g}, 57.8 \mathrm{mmol})$ in $50 \mathrm{~mL}$ dry dichloromethane was added dropwise to the above solution at $0{ }^{\circ} \mathrm{C}$ in 40 minutes. The reaction mixture was allowed to warm to room temperature slowly and stirred for 2 days under nitrogen. Upon completion of the reaction, the reaction mixture was washed by water $(50 \mathrm{~mL} * 3)$ and brine $(50 \mathrm{ml} * 1)$. The organic layer was dried over $\mathrm{Na}_{2} \mathrm{SO}_{4}$, filtered and concentrated under reduced pressure to afford the crude mixture which was further purified by column chromatography on silica gel to afford 1ac (3.0 g, 29\% for 3 steps) as a white solid. m. p.: $155-156{ }^{\circ} \mathrm{C} .{ }^{1} \mathbf{H}$ NMR $\left(400 \mathrm{MHz}, \mathrm{CDCl}_{3}\right) \delta 7.79(\mathrm{~d}, J=7.7 \mathrm{~Hz}, 2 \mathrm{H}), 7.46-7.40(\mathrm{~m}, 2 \mathrm{H}), 7.34(\mathrm{ddd}, J$ $=7.6,1.3,0.7 \mathrm{~Hz}, 2 \mathrm{H}), 7.28(\mathrm{dd}, J=7.6,1.3 \mathrm{~Hz}, 2 \mathrm{H}), 3.75-3.67(\mathrm{~m}, 4 \mathrm{H}), 1.25(\mathrm{~s}, 6 \mathrm{H}), 1.17(\mathrm{~s}, 6 \mathrm{H}) .{ }^{13} \mathrm{C}$ NMR $\left(101 \mathrm{MHz}, \mathrm{CDCl}_{3}\right) \delta 163.2,141.4,129.9,129.5,127.7,127.0,79.4,67.1,28.0,27.9$. HRMS calcd. for $\mathrm{C}_{22} \mathrm{H}_{25} \mathrm{~N}_{2} \mathrm{O}_{2}{ }^{+}[\mathrm{M}+\mathrm{H}]^{+}:$349.1911, found: 349.1918. IR $\left(\mathrm{cm}^{-1}\right)$ 3061, 2967, 2928, 2888, 1657, 1463, 1445 , $1351,1313,1213,1190,1055,1036$. 


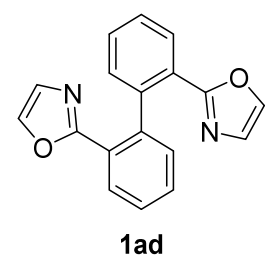

2-(2-iodophenyl)oxazole (4.0 g, $14.8 \mathrm{mmol})$ was subjected to general procedure $\mathbf{C}$ to afford $865.0 \mathrm{mg} \mathbf{1 a d}$ with $41 \%$ yield as a white solid. m. p. $137-139{ }^{\circ} \mathrm{C} .{ }^{1} \mathbf{H}$ NMR $\left(400 \mathrm{MHz}, \mathrm{CDCl}_{3}\right) \delta 8.04-7.97(\mathrm{~m}, 2 \mathrm{H})$, $7.50-7.43(\mathrm{~m}, 4 \mathrm{H}), 7.36(\mathrm{~s}, 2 \mathrm{H}), 7.32-7.24(\mathrm{~m}, 2 \mathrm{H}), 7.00(\mathrm{~s}, 2 \mathrm{H}) .{ }^{13} \mathbf{C} \mathbf{N M R}\left(101 \mathrm{MHz}, \mathrm{CDCl}_{3}\right) \delta 161.7$, $140.3,138.5,130.9,129.7,128.9,127.9,127.6,126.7$. HRMS calcd. for $\mathrm{C}_{18} \mathrm{H}_{13} \mathrm{~N}_{2} \mathrm{O}_{2}^{+}[\mathrm{M}+\mathrm{H}]^{+}:$289.0972, found: 289.0994. IR $\left(\mathrm{cm}^{-1}\right) 3160,3126,3060,3025,1601,1579,1557,1516,1478,1463,1444,1359$, 1245, 1139, 1106, 1076, 1005.

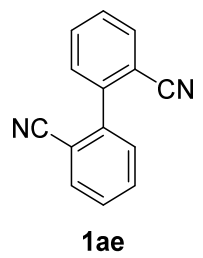

Substrate 1ae was synthesized by following the literature reported method ${ }^{9}{ }^{1} \mathbf{H}$ NMR $\left(400 \mathrm{MHz}, \mathrm{CDCl}_{3}\right) \delta$ $7.86-7.81(\mathrm{~m}, 2 \mathrm{H}), 7.77-7.69(\mathrm{~m}, 2 \mathrm{H}), 7.61-7.54(\mathrm{~m}, 4 \mathrm{H}) .{ }^{13} \mathbf{C} \mathbf{N M R}\left(101 \mathrm{MHz}, \mathrm{CDCl}_{3}\right) \delta 141.5$, $133.5,132.8,130.5,129.2,117.5,112.3$. Analytic data match the literature ${ }^{10}$.

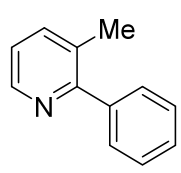

2a

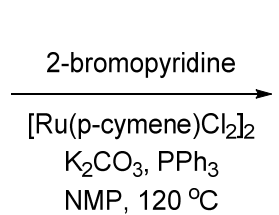
NMP, $120^{\circ} \mathrm{C}$

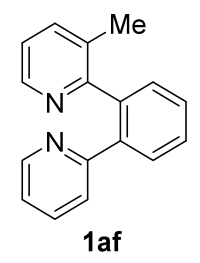

To a $40 \mathrm{~mL}$ vial charged with a stir bar, 3-methyl-2-phenylpyridine (1.69 g, $10.0 \mathrm{mmol})$, 2-bromopyridine (1.58 g, $10.0 \mathrm{mmol}), \mathrm{K}_{2} \mathrm{CO}_{3}(2.76 \mathrm{~g}, 20 \mathrm{mmol}), \mathrm{PPh}_{3}(262 \mathrm{mg}, 1.0 \mathrm{mmol})$ and $\left[\mathrm{Ru}(p \text {-cymene }) \mathrm{Cl}_{2}\right]_{2}(153.0 \mathrm{mg}, 0.25 \mathrm{mmol})$ were added. The vial was then transferred into the glovebox. Dry NMP $(20 \mathrm{~mL})$ was then added to the vial. The vial was then sealed and transferred out from the glovebox. The reaction mixture was then heated to $120{ }^{\circ} \mathrm{C}$ overnight. Upon completion of the reaction, the reaction mixture was diluted with $200 \mathrm{~mL}$ ethyl acetate and washed by water $(100 \mathrm{~mL} * 5)$ and brine $(50$ $\mathrm{mL} * 1$ ). The organic layer was dried over $\mathrm{Na}_{2} \mathrm{SO}_{4}$, filtered and concentrated under reduced pressure to afford the crude mixture, which was further purified by column chromatography on silica gel to afford 1af (441.9 mg, $18 \%)$ as a colorless oil. ${ }^{1} \mathbf{H}$ NMR $\left(400 \mathrm{MHz}, \mathrm{CDCl}_{3}\right) \delta 8.60-8.53(\mathrm{~m}, 1 \mathrm{H}), 8.49(\mathrm{dd}, J=4.8$, $1.0 \mathrm{~Hz}, 1 \mathrm{H}), 7.81-7.73(\mathrm{~m}, 1 \mathrm{H}), 7.57-7.46(\mathrm{~m}, 2 \mathrm{H}), 7.43-7.37$ (m, 1H), $7.36-7.28(\mathrm{~m}, 2 \mathrm{H}), 7.10(\mathrm{dd}$, $J=7.7,4.8 \mathrm{~Hz}, 1 \mathrm{H}), 7.05(\mathrm{ddd}, J=7.5,4.9,1.1 \mathrm{~Hz}, 1 \mathrm{H}), 6.85(\mathrm{dd}, J=7.9,0.9 \mathrm{~Hz}, 1 \mathrm{H}), 1.75(\mathrm{~s}, 3 \mathrm{H}) .{ }^{13} \mathbf{C}$ NMR $\left(101 \mathrm{MHz}, \mathrm{CDCl}_{3}\right) \delta 159.3,158.4,149.5,146.6,139.5,139.3,137.5,135.2,131.9,129.7,129.6$, 
128.6, 128.4, 124.3, 122.2, 121.4, 18.8. HRMS calcd. for $\mathrm{C}_{17} \mathrm{H}_{15} \mathrm{~N}_{2}^{+}[\mathrm{M}+\mathrm{H}]^{+}:$247.1230, found: 247.1251 . IR $\left(\mathrm{cm}^{-1}\right) 3054,3006,2925,2866,1585,1566,1493,1463,1419,1381,1300,1289,1269,1234,1184$, 1151, 1094.

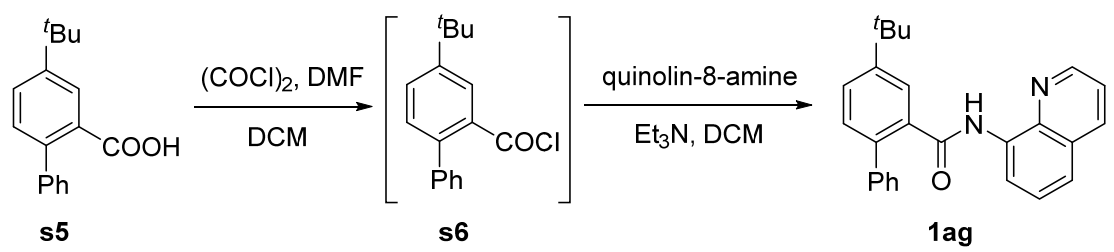

To a solution of 4-(tert-butyl)-[1,1'-biphenyl]-2-carboxylic acid s5 (2.85 g, $11.21 \mathrm{mmol})$ in $50 \mathrm{~mL}$ dry dichloromethane in a $100 \mathrm{~mL}$ schlenk flask, 5 drops of dry DMF was added. Oxalyl chloride (2.85 g, 22.45 mmol) was subsequently added dropwise to the above suspension. The resulting reaction mixture was stirred at room temperature for $6 \mathrm{~h}$. Upon completion of the reaction, the solvent was removed under reduced pressure to afford the acyl chloride s6 which was used directly for the next step. The crude acyl chloride s6 was then dissolved in dichloromethane $(30 \mathrm{~mL})$ and slowly added to a stirred solution of quinoline-8-amine $(1.6 \mathrm{~g}, 11.21 \mathrm{mmol})$ and $\mathrm{Et}_{3} \mathrm{~N}(3.1 \mathrm{~mL})$ in dichloromethane $(30 \mathrm{~mL})$ at $0{ }^{\circ} \mathrm{C}$ over 30 min. The reaction mixture was allowed to warm to room temperature slowly and stirred overnight under nitrogen. Upon completion of the reaction, the reaction mixture was washed by water $(50 \mathrm{~mL} * 3)$ and brine $(50 \mathrm{ml} * 1)$. The organic layer was dried over $\mathrm{Na}_{2} \mathrm{SO}_{4}$, filtered and concentrated under reduced pressure to afford the crude mixture which was further purified by column chromatography on silica gel to afford 1ag (2.48 g, 58\%) as a light yellow solid. m.p. $141-142{ }^{\circ} \mathrm{C} .{ }^{1} \mathbf{H}$ NMR $\left(400 \mathrm{MHz}, \mathrm{CDCl}_{3}\right) \delta 9.74(\mathrm{~s}$, $1 \mathrm{H}), 8.83(\mathrm{dd}, J=7.6,1.2 \mathrm{~Hz}, 1 \mathrm{H}), 8.48(\mathrm{dd}, J=4.2,1.7 \mathrm{~Hz}, 1 \mathrm{H}), 8.04(\mathrm{dd}, J=8.3,1.7 \mathrm{~Hz}, 1 \mathrm{H}), 7.94(\mathrm{~d}, J$ $=2.1 \mathrm{~Hz}, 1 \mathrm{H}), 7.59(\mathrm{dd}, J=8.1,2.1 \mathrm{~Hz}, 1 \mathrm{H}), 7.55-7.46(\mathrm{~m}, 3 \mathrm{H}), 7.46-7.39(\mathrm{~m}, 2 \mathrm{H}), 7.31(\mathrm{dd}, J=8.3$, $4.2 \mathrm{~Hz}, 1 \mathrm{H}), 7.28-7.21(\mathrm{~m}, 2 \mathrm{H}), 7.16-7.08(\mathrm{~m}, 1 \mathrm{H}), 1.41(\mathrm{~s}, 9 \mathrm{H}) .{ }^{13} \mathbf{C} \mathbf{N M R}\left(101 \mathrm{MHz}, \mathrm{CDCl}_{3}\right) \delta 168.4$, $150.7,147.6,139.9,138.4,137.3,135.9,135.7,134.6,130.4,129.0,128.3,127.7,127.6,127.4,127.2$, 126.2, 121.4, 121.3, 116.1, 34.7, 31.3. HRMS calcd. for $\mathrm{C}_{26} \mathrm{H}_{25} \mathrm{~N}_{2} \mathrm{O}^{+}[\mathrm{M}+\mathrm{H}]^{+}: 381.1961$, found: 381.1979 . IR $\left(\mathrm{cm}^{-1}\right)$ 3324, 3053, 2962, 2903, 2867, 1667, 1596, 1578, 1525, 1484, 1444, 1424, 1385, 1327, 1228.

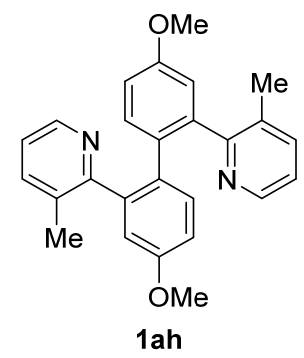

2-(3-methoxyphenyl)-3-methylpyridine $(6.55 \mathrm{~g}, 32.87 \mathrm{mmol})$ was subjected to general procedure $\mathbf{A}$ to afford $1.62 \mathrm{~g}$ 1ah with $25 \%$ yield as a white solid. m.p. $123-125{ }^{\circ} \mathrm{C} .{ }^{1} \mathrm{H}$ NMR $\left(400 \mathrm{MHz}, \mathrm{DMSO}-\mathrm{d}_{6}\right) \delta$ $8.21(\mathrm{~s}, 2 \mathrm{H}), 7.51$ (d, $J=7.3 \mathrm{~Hz}, 2 \mathrm{H}), 7.13$ (dd, $J=7.6,4.8 \mathrm{~Hz}, 2 \mathrm{H}), 6.91$ (brs, 1H), $6.78-6.59$ (m, 4H), 3.67 (s, 6H), 1.98 (brs, 6H). ${ }^{13}$ C NMR (125 MHz, DMSO-d 6,343 K) $\delta$ 158.3, 156.9, 145.3, 140.4, 137.1, 
132.3, 131.3, 131.1, 121.4, 114.6, 112.3, 54.7, 18.7. HRMS calcd. for $\mathrm{C}_{26} \mathrm{H}_{25} \mathrm{~N}_{2} \mathrm{O}_{2}{ }^{+}[\mathrm{M}+\mathrm{H}]^{+}: 397.1911$, found: 397.1931. IR $\left(\mathrm{cm}^{-1}\right)$ 3048, 3000, 2956, 2835, 1605, 1583, 1559, 1480, 1464, 1428, 1317, 1296, $1266,1216,1175,1115$.

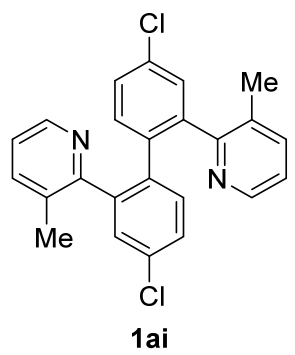

2-(3-chlorophenyl)-3-methylpyridine (3.33 g, $16.35 \mathrm{mmol})$ was subjected to general procedure A to afford $839.2 \mathrm{mg}$ 1aj with $25 \%$ yield as a white solid. m.p. $222-123{ }^{\circ} \mathrm{C} .{ }^{1} \mathbf{H}$ NMR $(400 \mathrm{MHz}$, DMSO-d 6 ) $\delta$ $8.18(\mathrm{~s}, 2 \mathrm{H}), 7.54(\mathrm{~d}, J=7.5 \mathrm{~Hz}, 2 \mathrm{H}), 7.31(\mathrm{~d}, J=8.0 \mathrm{~Hz}, 2 \mathrm{H}), 7.23(\mathrm{~d}, J=2.0 \mathrm{~Hz}, 2 \mathrm{H}), 7.20-7.05(\mathrm{~m}$, 4H), 1.92 (brs, 6H). ${ }^{13}$ C NMR (125 MHz, DMSO-d 6 , 343 K) $\delta 160.1,156.5,145.4,141.2,137.4,134.7$, 133.2, 131.2, 121.9, 115.7, 113.2, 18.4. HRMS calcd. for $\mathrm{C}_{24} \mathrm{H}_{19} \mathrm{Cl}_{2} \mathrm{~N}_{2}{ }^{+}[\mathrm{M}+\mathrm{H}]^{+}$: 405.0920, found: 405.0933. IR $\left(\mathrm{cm}^{-1}\right)$ 3049, 2955, 2927, 2356, 1583, 1570, 1470, 1462, 1444, 1426, 1381, 1289, 1265, 1183 , 1116. 


\section{Aryl-Aryl bond activation}

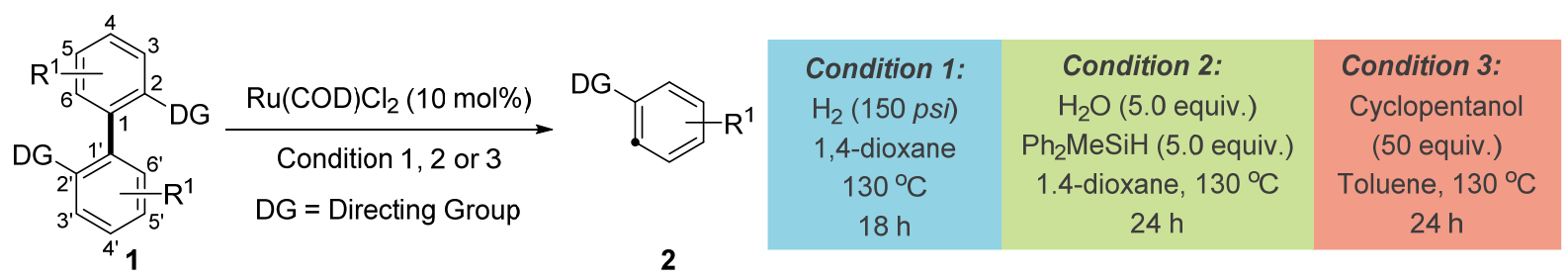

Condition 1: To a Q-tube charged with a stir bar, substrates (1.0 equiv. 0.1 - $0.2 \mathrm{mmol})$ and $\mathrm{Ru}(\mathrm{COD}) \mathrm{Cl}_{2}(10$ mol\%) were added. The Q-tube was then transferred into the glovebox. Distilled 1,4-dioxane (0.075 M) was added. The Q-tube was reassembled and transferred out of the glovebox. The reaction mixture was in situ freeze-pump-thawed using standard technique for three times. The Q-tube was then charged with 150 psi hydrogen gas followed by heating at $130{ }^{\circ} \mathrm{C}$ in a pre-heated oil bath for $18 \mathrm{~h}$. Upon completion of the reaction, the reaction mixture was transferred in to a flask, concentrated under reduced pressure and directly purified using column chromatography on silica gel to afford the desired product.

Condition 2: To a flame dried $4 \mathrm{~mL}$ vial charged with a stir bar, substrates (1.0 equiv. 0.1 - $0.2 \mathrm{mmol})$ and $\mathrm{Ru}(\mathrm{COD}) \mathrm{Cl}_{2}(10 \mathrm{~mol} \%)$ were added. The vial was transferred to the glovebox. $\mathrm{Ph}_{2} \mathrm{MeSiH}_{(5.0}$ equiv.), $\mathrm{H}_{2} \mathrm{O}$ (5.0 equiv.) and distilled 1,4-dioxane $(1.0 \mathrm{~mL} / 0.1 \mathrm{mmol} \mathrm{1)}$ were added. The vial was sealed and stirred on a pre-heated heating block at $130{ }^{\circ} \mathrm{C}$ for $24 \mathrm{~h}$. Upon completion of the reaction, the reaction mixture was transferred in to a flask, concentrated under reduced pressure and directly purified using column chromatography on silica gel to afford the desired product. (For product $\mathbf{2 k}$ obtained by this condition, it was not completely pure after column chromatography on silica gel and PTLC. The yield was determined by ${ }^{1} \mathrm{H}$ NMR using 1,1,2,2-tetrachloroethane as the internal standard after column chromatography on silica gel.)

Condition 3: To a flame dried $4 \mathrm{~mL}$ vial charged with a stir bar, substrates (1.0 equiv. $0.1-0.2 \mathrm{mmol})$ and $\mathrm{Ru}(\mathrm{COD}) \mathrm{Cl}_{2}$ (10 mol\%) were added. The vial was transferred to the glovebox. Cyclopentanol (50 equiv.) was added followed by the addition of distilled toluene $(1.0 \mathrm{~mL} / 0.1 \mathrm{mmol} \mathrm{1})$. The vial was sealed and stirred at a pre-heated heating block at $130{ }^{\circ} \mathrm{C}$ for $24 \mathrm{~h}$. Upon completion of the reaction, the reaction mixture was transferred in to a flask, concentrated under reduced pressure and directly purified using column chromatography on silica gel to afford the desired product.

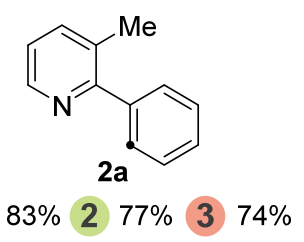


All three conditions were run with $0.1 \mathrm{mmol}$ 1a. 2 a: light yellow oil. ${ }^{\mathbf{1}} \mathbf{H} \mathbf{N M R}\left(400 \mathrm{MHz}, \mathrm{CDCl}_{3}\right) \delta 8.51(\mathrm{dd}, J$ $=4.7,1.1 \mathrm{~Hz}, 1 \mathrm{H}), 7.55(\mathrm{dd}, J=7.7,0.8 \mathrm{~Hz}, 1 \mathrm{H}), 7.43(\mathrm{~d}, J=8.1 \mathrm{~Hz}, 2 \mathrm{H}), 7.25(\mathrm{~d}, J=7.8 \mathrm{~Hz}, 2 \mathrm{H}), 7.14(\mathrm{dd}, J$ $=7.7,4.8 \mathrm{~Hz}, 1 \mathrm{H}), 2.40(\mathrm{~s}, 3 \mathrm{H}), 2.35$ (s, 3H). ${ }^{13} \mathbf{C} \mathbf{N M R}\left(100 \mathrm{MHz}, \mathrm{CDCl}_{3}\right) \delta 158.6,146.9,140.5,138.4,130.7$, 128.8, 128.0, 127.8, 122.0, 20.0. HRMS calcd. for $\mathrm{C}_{13} \mathrm{H}_{14} \mathrm{~N}^{+}[\mathrm{M}+\mathrm{H}]^{+}:$184.1121, found: 184.1124. IR $\left(\mathrm{cm}^{-1}\right)$ 3049, 2921, 1584, 1515, 1446, 1424, 1182, 1119, 1110, 1037, 1017.

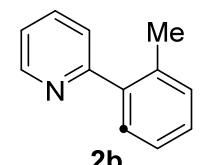

$35 \% \quad 2 \quad 23 \% \quad 3 \quad 39 \%$

All three conditions were run with $0.1 \mathrm{mmol}$ 1b. 2b: Colorless oil. ${ }^{1} \mathrm{H}$ NMR $\left(400 \mathrm{MHz}, \mathrm{CDCl}_{3}\right) \delta 8.76-8.67$ (m, 1H), $7.75(\mathrm{td}, J=7.7,1.8 \mathrm{~Hz}, 1 \mathrm{H}), 7.45-7.39(\mathrm{~m}, 2 \mathrm{H}), 7.36-7.21(\mathrm{~m}, 4 \mathrm{H}), 2.39(\mathrm{~s}, 3 \mathrm{H}) .{ }^{13} \mathbf{C}$ NMR $(100$ $\left.\mathrm{MHz}, \mathrm{CDCl}_{3}\right) \delta 160.0,149.2,140.4,136.0,135.7,130.7,129.6,128.2,125.8,124.0,121.6,20.2$. Analytic data match the literature ${ }^{11}$.

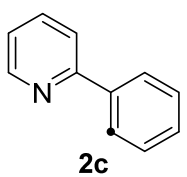

(1) $79 \%$ 2 $77 \%$ (3) $56 \%$

Condition 1: 0.2 mmol 1c; condition 2: $0.1 \mathrm{mmol} \mathrm{1c;} \mathrm{condition} \mathrm{3:} 0.13 \mathrm{mmol}$ 1c. $2 \mathrm{c}$ : Colorless oil. ${ }^{\mathbf{1}} \mathbf{H}$ NMR $\left(400 \mathrm{MHz} \mathrm{CDCl}_{3}\right) \delta 8.72-8.69(\mathrm{~m}, 1 \mathrm{H}), 8.01-7.97(\mathrm{~m}, 2 \mathrm{H}), 7.78-7.71(\mathrm{~m}, 2 \mathrm{H}), 7.51-7.46(\mathrm{~m}, 2 \mathrm{H}), 7.44$ $-7.39(\mathrm{~m}, 1 \mathrm{H}), 7.25-7.21(\mathrm{~m}, 1 \mathrm{H}) .{ }^{13} \mathbf{C} \mathbf{N M R}\left(101 \mathrm{MHz}, \mathrm{CDCl}_{3}\right) \delta 157.4,149.6,139.4,136.7,128.9,128.7$, 126.9, 122.1, 120.6. HRMS calcd. for $\mathrm{C}_{11} \mathrm{H}_{10} \mathrm{~N}^{+}[\mathrm{M}+\mathrm{H}]^{+}: 156.0808$, found: 156.0811. IR ( $\left.\mathrm{cm}^{-1}\right) 3062,1587$, $1565,1468,1449,1425,1152,1074,1020$.

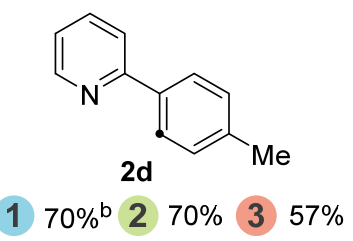

Condition 1: $0.2 \mathrm{mmol} \mathrm{1d}$; condition 2: $0.1 \mathrm{mmol} \mathrm{1d}$; condition 3: $0.2 \mathrm{mmol} \mathrm{1d.} \mathrm{2d:} \mathrm{Colorless} \mathrm{oil.}{ }^{\mathbf{1}} \mathbf{H}$ NMR $\left(400 \mathrm{MHz}, \mathrm{CDCl}_{3}\right) \delta 8.69-8.66(\mathrm{~m}, 1 \mathrm{H}), 7.92-7.87(\mathrm{~m}, 2 \mathrm{H}), 7.76-7.68(\mathrm{~m}, 2 \mathrm{H}), 7.30-7.26(\mathrm{~m}, 2 \mathrm{H}), 7.20$ $(\mathrm{ddd}, J=6.7,4.8,2.0 \mathrm{~Hz}, 1 \mathrm{H}), 2.41(\mathrm{~s}, 3 \mathrm{H}) .{ }^{13} \mathbf{C} \mathbf{N M R}\left(101 \mathrm{MHz}, \mathrm{CDCl}_{3}\right) \delta 157.4,149.6,138.9,136.7,136.6$, 129.5, 126.8, 121.8, 120.3, 21.3. HRMS calcd. for $\mathrm{C}_{12} \mathrm{H}_{12} \mathrm{~N}^{+}[\mathrm{M}+\mathrm{H}]^{+}: 170.0964$, found: 170.0969 . IR $\left(\mathrm{cm}^{-1}\right)$ 1615, 1588, 1563, 1467, 1433, 1185, 1153, 1094. 


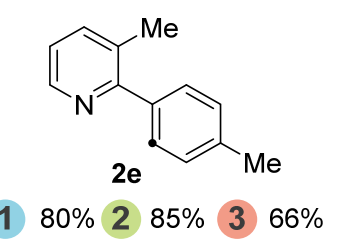

Condition 1: $0.2 \mathrm{mmol} \mathrm{1e}$; condition 2: $0.1 \mathrm{mmol} \mathrm{1e}$; condition 3: $0.2 \mathrm{mmol} \mathrm{1e.} \mathrm{2e:} \mathrm{Colorless} \mathrm{oil.}{ }^{1} \mathbf{H}$ NMR (400 $\left.\mathrm{MHz}, \mathrm{CDCl}_{3}\right) \delta 8.51(\mathrm{dd}, J=4.7,1.1 \mathrm{~Hz}, 1 \mathrm{H}), 7.55(\mathrm{dd}, J=7.7,0.8 \mathrm{~Hz}, 1 \mathrm{H}), 7.43(\mathrm{~d}, J=8.1 \mathrm{~Hz}, 2 \mathrm{H}), 7.25(\mathrm{~d}, J$ $=7.8 \mathrm{~Hz}, 2 \mathrm{H}), 7.14(\mathrm{dd}, J=7.7,4.8 \mathrm{~Hz}, 1 \mathrm{H}), 2.40(\mathrm{~s}, 3 \mathrm{H}), 2.35(\mathrm{~s}, 3 \mathrm{H}) .{ }^{13} \mathbf{C} \mathbf{N M R}\left(101 \mathrm{MHz}, \mathrm{CDCl}_{3}\right) \delta 158.6$, $146.8,138.3,137.7,137.5,130.6,128.7,128.7,121.7,21.2,20.1$. HRMS calcd. for $\mathrm{C}_{13} \mathrm{H}_{14} \mathrm{~N}^{+}[\mathrm{M}+\mathrm{H}]^{+}$: 184.1121, found: 184.1124. IR $\left(\mathrm{cm}^{-1}\right)$ 3049, 2921, 1584, 1515, 1446, 1424, 1182, 1119, 1110, 1037, 1017.

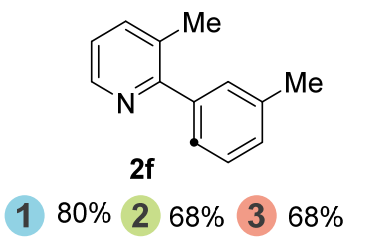

Condition 1: $0.1 \mathrm{mmol}$ 1f; condition 2: $0.1 \mathrm{mmol} \mathrm{1f;}$ condition 3: $0.2 \mathrm{mmol} \mathrm{1f.} \mathrm{2f:} \mathrm{Colorless} \mathrm{oil.}{ }^{1} \mathbf{H}$ NMR (500 $\left.\mathrm{MHz}, \mathrm{CDCl}_{3}\right) \delta 8.52(\mathrm{~d}, J=4.0 \mathrm{~Hz}, 1 \mathrm{H}), 7.55(\mathrm{~d}, J=8.0 \mathrm{~Hz}, 1 \mathrm{H}), 7.35-7.29(\mathrm{~m}, 3 \mathrm{H}), 7.21-7.17(\mathrm{~m}, 1 \mathrm{H})$, $7.16-7.15(\mathrm{~m}, 1 \mathrm{H}), 2.41(\mathrm{~s}, 3 \mathrm{H}), 2.35(\mathrm{~s}, 3 \mathrm{H}) .{ }^{13} \mathbf{C}$ NMR $\left(125 \mathrm{MHz}, \mathrm{CDCl}_{3}\right) \delta$ 158.8, 146.8, 140.5, 138.4, 137.8, 130.7, 129.6, 128.6, 127.8, 125.9, 121.9, 21.4, 20.0. HRMS calcd. for $\mathrm{C}_{13} \mathrm{H}_{14} \mathrm{~N}^{+}[\mathrm{M}+\mathrm{H}]^{+}:$184.1121, found: 184.1127. IR $\left(\mathrm{cm}^{-1}\right) 3045,2955,2921,2863,1608,1583,1566,1445,1382,1200,1178,1119,1093$, $1065,1039$.

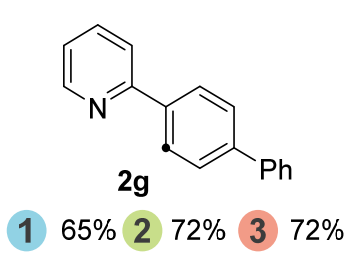

Condition 1: $0.1 \mathrm{mmol} \mathrm{1g}$; condition 2: $0.1 \mathrm{mmol} \mathrm{1g}$; condition 3: $0.1 \mathrm{mmol} 1 \mathrm{~g}$. 2g: White solid. m.p. 143-144 ${ }^{\circ} \mathrm{C} .{ }^{1} \mathbf{H}$ NMR $\left(400 \mathrm{MHz}, \mathrm{CDCl}_{3}\right) \delta 8.70(\mathrm{dt}, J=4.8,1.5 \mathrm{~Hz}, 1 \mathrm{H}), 8.10-8.04(\mathrm{~m}, 2 \mathrm{H}), 7.78-7.74(\mathrm{~m}, 2 \mathrm{H}), 7.73$ - $7.68(\mathrm{~m}, 2 \mathrm{H}), 7.65(\mathrm{dt}, J=3.1,1.9 \mathrm{~Hz}, 2 \mathrm{H}), 7.48-7.42(\mathrm{~m}, 2 \mathrm{H}), 7.39-7.33(\mathrm{~m}, 1 \mathrm{H}), 7.25-7.21(\mathrm{~m}, 1 \mathrm{H})$. ${ }^{13}$ C NMR (101 MHz, $\left.\mathrm{CDCl}_{3}\right) \delta 157.0,149.7,141.7,140.5,138.2,136.8,128.8,127.5,127.4,127.3,127.1$, 122.1, 120.4. HRMS calcd. for $\mathrm{C}_{17} \mathrm{H}_{14} \mathrm{~N}^{+}[\mathrm{M}+\mathrm{H}]^{+}:$232.1121, found: 232.1128. IR $\left(\mathrm{cm}^{-1}\right)$ 1582, 1571, 1465, $1449,1430,1403,909$.

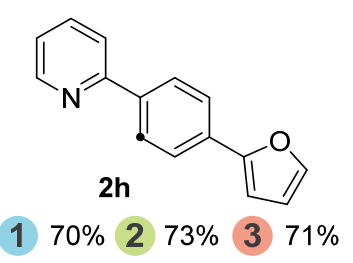


All three conditions were run with $0.1 \mathrm{mmol} \mathbf{1 h} . \mathbf{2 h}$ : Pale yellow solid. m.p. $135-136{ }^{\circ} \mathrm{C} .{ }^{\mathbf{1}} \mathbf{H}$ NMR $(500 \mathrm{MHz}$, $\left.\mathrm{CDCl}_{3}\right) \delta 8.70-8.69(\mathrm{~m}, 1 \mathrm{H}), 8.05-8.03(\mathrm{~m}, 2 \mathrm{H}), 7.79-7.77(\mathrm{~m}, 2 \mathrm{H}), 7.75-7.73(\mathrm{~m}, 2 \mathrm{H}), 7.51-7.50(\mathrm{~m}$, 1H), $7.23-7.21(\mathrm{~m}, 1 \mathrm{H}), 6.74-6.73(\mathrm{~m}, 1 \mathrm{H}), 6.51-6.50(\mathrm{~m}, 1 \mathrm{H}) .{ }^{13} \mathbf{C} \mathbf{~ N M R}\left(101 \mathrm{MHz}, \mathrm{CDCl}_{3}\right) \delta 156.8$, 153.6, 149.7, 142.3, 138.0, 136.7, 131.3, 127.1, 124.0, 122.1, 120.3, 111.8, 105.6. HRMS calcd. for $\mathrm{C}_{15} \mathrm{H}_{12} \mathrm{NO}^{+}[\mathrm{M}+\mathrm{H}]^{+}:$222.0913, found: 222.0919. IR $\left(\mathrm{cm}^{-1}\right) 3120,3103,3056,1607,1585,1514,1481,1463$, 1435, 1409, 1374, 1281, 1264, 1218, 1186, 1155, 1114, 1075, 1046, 1006.

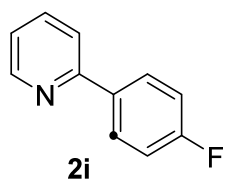

(1) $71 \% 270 \% \quad 3 \quad 50 \%$

Condition 1: $0.15 \mathrm{mmol} \mathrm{1i}$; condition 2: $0.2 \mathrm{mmol} \mathrm{1i}$; condition 3: $0.2 \mathrm{mmol} \mathbf{1 i}$. $2 \mathbf{i}$ : White solid. ${ }^{1} \mathbf{H}$ NMR (400 $\left.\mathrm{MHz}, \mathrm{CDCl}_{3}\right) \delta 8.68-8.63(\mathrm{~m}, 1 \mathrm{H}), 8.00-7.92(\mathrm{~m}, 2 \mathrm{H}), 7.73(\mathrm{td}, J=7.9,1.7 \mathrm{~Hz}, 1 \mathrm{H}), 7.66(\mathrm{~d}, J=8.0 \mathrm{~Hz}$, 1H), $7.23-7.19(\mathrm{~m}, 1 \mathrm{H}), 7.18-7.10(\mathrm{~m}, 2 \mathrm{H}) .{ }^{13} \mathbf{C} \mathbf{~ N M R}\left(101 \mathrm{MHz}, \mathrm{CDCl}_{3}\right) \delta 163.6(\mathrm{~d}, J=250 \mathrm{~Hz}), 156.4$, 149.6, 136.8, 135.5 (d, $J=3.2 \mathrm{~Hz}), 128.7$ (d, $J=8.3 \mathrm{~Hz}), 122.0,120.2,115.6$ (d, $J=21.6 \mathrm{~Hz})$. HRMS calcd. for $\mathrm{C}_{11} \mathrm{H}_{9} \mathrm{FN}^{+}[\mathrm{M}+\mathrm{H}]^{+}: 174.0714$, found: 174.0716. IR $\left(\mathrm{cm}^{-1}\right) 3054,1600,1585,1568,1511,1467,1436,1408$, $1299,1225,1152,1165,1100$. Analytic data match literature ${ }^{12}$.

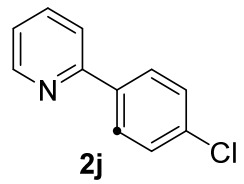

(1) $70 \% \quad 2 \quad 54 \% \quad 3 \quad 80 \%$

All three conditions were run with $0.1 \mathrm{mmol} \mathbf{1 j}$. $\mathbf{2 j}$ : White solid. ${ }^{1} \mathbf{H}$ NMR $\left(400 \mathrm{MHz}, \mathrm{CDCl}_{3}\right) \delta 8.70-8.68(\mathrm{~m}$, 1H), $7.96-7.93(\mathrm{~m}, 2 \mathrm{H}), 7.78-7.68(\mathrm{~m}, 2 \mathrm{H}), 7.47-7.43(\mathrm{~m}, 2 \mathrm{H}), 7.27-7.23(\mathrm{~m}, 1 \mathrm{H}) .{ }^{13} \mathbf{C}$ NMR $(125 \mathrm{MHz}$, $\left.\mathrm{CDCl}_{3}\right) \delta 156.1,149.7,137.7,136.8,135.0,128.9,128.1,122.3,120.3$. Analytic data match literature ${ }^{12}$.

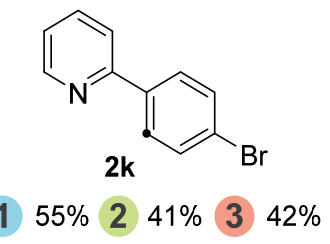

Condition 1: $0.1 \mathrm{mmol} \mathrm{1k}$; condition 2: $0.2 \mathrm{mmol} \mathbf{1 k}$; condition 3: $0.2 \mathrm{mmol} \mathbf{1 k}$. 2k: White solid. ${ }^{1} \mathbf{H}$ NMR $\left(500 \mathrm{MHz}, \mathrm{CDCl}_{3}\right) \delta 8.69-8.67(\mathrm{~m}, 1 \mathrm{H}), 7.88-7.86(\mathrm{~m}, 2 \mathrm{H}), 7.76-7.73(\mathrm{~m}, 1 \mathrm{H}), 7.69-7.68(\mathrm{~m}, 1 \mathrm{H}), 7.59$ - 7.58 (m, 2H), 7.27 - 7.23 (m, 1H). ${ }^{13} \mathbf{C}$ NMR $\left(125 \mathrm{MHz}, \mathrm{CDCl}_{3}\right) \delta 156.2,149.7,138.2,136.8,131.8,128.4$, 123.4, 122.4, 120.3. Analytic data match literature ${ }^{13}$. 


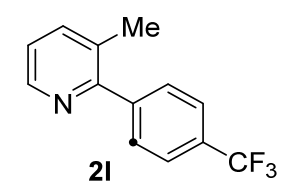

(1) $62 \%^{\mathrm{c}} 273 \%$ (3) $83 \%$

Condition 1: $0.1 \mathrm{mmol} \mathrm{11,} \mathrm{3h;} \mathrm{condition} \mathrm{2:} 0.2 \mathrm{mmol} \mathrm{1l;} \mathrm{condition} \mathrm{3:} 0.2 \mathrm{mmol} \mathrm{11.} \mathrm{21:} \mathrm{Colorless} \mathrm{oil.}{ }^{1} \mathbf{H}$ NMR $\left(500 \mathrm{MHz}, \mathrm{CDCl}_{3}\right){ }^{1} \mathbf{H} \mathbf{N M R}\left(500 \mathrm{MHz}, \mathrm{CDCl}_{3}\right) \delta 8.53(\mathrm{~d}, J=3.9 \mathrm{~Hz}, 1 \mathrm{H}), 7.70(\mathrm{~d}, J=8.2 \mathrm{~Hz}, 2 \mathrm{H}), 7.64(\mathrm{~d}, J$ $=8.1 \mathrm{~Hz}, 2 \mathrm{H}), 7.61-7.57(\mathrm{~m}, 1 \mathrm{H}), 7.21(\mathrm{dd}, J=7.7,4.8 \mathrm{~Hz}, 1 \mathrm{H}), 2.34(\mathrm{~s}, 3 \mathrm{H}) \cdot{ }^{13} \mathbf{C} \mathbf{~ N M R}\left(125 \mathrm{MHz}, \mathrm{CDCl}_{3}\right)$ $\delta$ 157.1, 147.1, 144.1, 138.7, 130.9, $129.9(\mathrm{q}, J=40.3 \mathrm{~Hz}), 129.3,125.0(\mathrm{q}, J=338.0 \mathrm{~Hz}), 124.1(\mathrm{q}, J=4.3$ $\mathrm{Hz}), 122.7,19.8 .{ }^{19} \mathbf{F}$ NMR $\left(376 \mathrm{MHz}, \mathrm{CDCl}_{3}\right) \delta-62.5$. HRMS calcd. for $\mathrm{C}_{13} \mathrm{H}_{10} \mathrm{~F}_{3} \mathrm{~N}^{+}[\mathrm{M}+\mathrm{H}]^{+}: 238.0838$, found: 238.0847 . IR $\left(\mathrm{cm}^{-1}\right)$ 3053, 2961, 2933, 2868, 1618, 1580, 1566, 1521, 1451, 1425, 1405, 1383, 1325, $1221,1164,1125,1071,1034$.

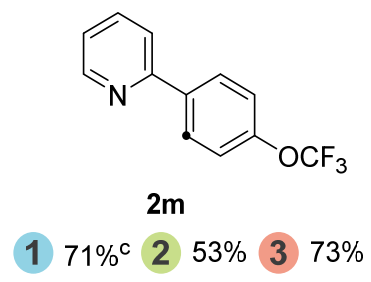

White solid. m.p. $56-58{ }^{\circ} \mathrm{C} .{ }^{1} \mathbf{H}$ NMR $\left(500 \mathrm{MHz}, \mathrm{CDCl}_{3}\right) \delta 8.72-8.68(\mathrm{~m}, 1 \mathrm{H}), 8.03-8.00(\mathrm{~m}, 2 \mathrm{H}), 7.76-$ $7.28(\mathrm{~m}, 1 \mathrm{H}), 7.69(\mathrm{~d}, J=8.0 \mathrm{~Hz}, 1 \mathrm{H}), 7.31(\mathrm{~d}, J=8.0 \mathrm{~Hz}, 2 \mathrm{H}), 7.25-7.23(\mathrm{~m}, 1 \mathrm{H}) .{ }^{13} \mathbf{C}$ NMR $(125 \mathrm{MHz}$, $\left.\mathrm{CDCl}_{3}\right) \delta 160.0,149.8,149.7,138.0,136.8,128.3,122.4,121.0,120.5(\mathrm{q}, \mathrm{J}=256 \mathrm{~Hz}), 120.4 .{ }^{19} \mathbf{F}$ NMR $(470$ $\left.\mathrm{MHz}, \mathrm{CDCl}_{3}\right) \delta$-57.7. HRMS calcd. for $\mathrm{C}_{12} \mathrm{H}_{9} \mathrm{~F}_{3} \mathrm{NO}^{+}[\mathrm{M}+\mathrm{H}]^{+}: 240.0631$, found: 240.0637. IR $\left(\mathrm{cm}^{-1}\right) 3063$, 3016, 2924, 1594, 1568, 1510, 1468, 1439, 1406, 1305, 1209, 1151m 1104, 1059, 1030, 1014.

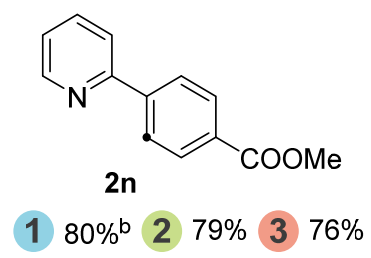

All three conditions were run with $0.1 \mathrm{mmol} \mathbf{1 n}$. The reaction time for condition 1 was $6 \mathrm{~h}$. $\mathbf{2 n}$ : White solid. ${ }^{1} \mathbf{H}$ NMR $\left(400 \mathrm{MHz}, \mathrm{CDCl}_{3}\right) \delta 8.73-8.70(\mathrm{~m}, 1 \mathrm{H}), 8.15-8.12(\mathrm{~m}, 2 \mathrm{H}), 8.08-8.05(\mathrm{~m}, 2 \mathrm{H}), 7.78-7.77(\mathrm{~m}, 2 \mathrm{H})$, $7.29-7.26(\mathrm{~m}, 1 \mathrm{H}), 3.94(\mathrm{~s}, 3 \mathrm{H}){ }^{13} \mathbf{C}$ NMR $\left(125 \mathrm{MHz}, \mathrm{CDCl}_{3}\right) \delta 166.9,156.1,149.8,143.5,136.9,130.3$, $130.0,126.8,122.8,121.0,52.1$. Analytic data match literature ${ }^{14}$.

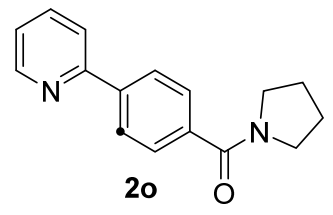

(1) $77 \% \quad 272 \% \quad 360 \%$ 
All three conditions were run with 0.1 mmol 1n. 2n: Colorless oil. ${ }^{1} \mathbf{H}$ NMR $\left(500 \mathrm{MHz}, \mathrm{CDCl}_{3}\right) \delta 8.66-8.65$ $(\mathrm{m}, 1 \mathrm{H}), 8.00-7.98(\mathrm{~m}, 2 \mathrm{H}), 7.74-7.69(\mathrm{~m}, 2 \mathrm{H}), 7.61-7.58(\mathrm{~m}, 2 \mathrm{H}), 7.22-7.20(\mathrm{~m}, 1 \mathrm{H}), 3.62(\mathrm{t}, J=7.0 \mathrm{~Hz}$, 2H), $3.41(\mathrm{t}, J=7.0 \mathrm{~Hz}, 2 \mathrm{H}), 1.95-1.89(\mathrm{~m}, 2 \mathrm{H}), 1.86-1.81(\mathrm{~m}, 2 \mathrm{H}) .{ }^{13} \mathbf{C} \mathbf{N M R}\left(101 \mathrm{MHz}, \mathrm{CDCl}_{3}\right) \delta 169.2$, $156.4,149.6,140.5,137.4,136.7,127.5,126.6,122.4,120.6,49.4,46.1,26.3,24.3$. Analytic data match literature $^{15}$.
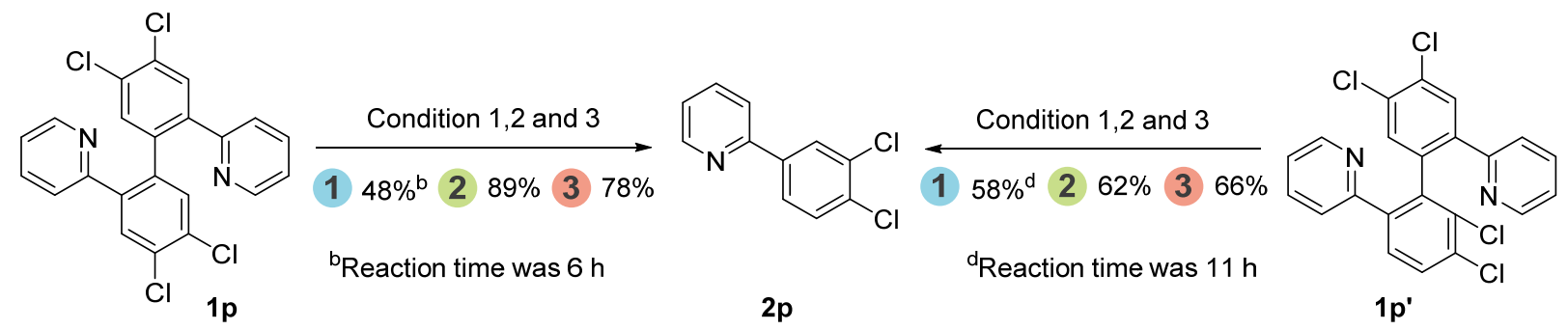

All three conditions were run with $0.1 \mathrm{mmol}$ 1p. The reaction for condition 1 with $1 \mathbf{p}$ was $6 \mathrm{~h}$.

All three conditions were run with 0.1 mmol 1p'. The reaction for condition 1 with $1 \mathbf{p}$ ' was $11 \mathrm{~h}$.

2p: White solid. ${ }^{1} \mathbf{H}$ NMR (500 MHz, $\left.\mathrm{CDCl}_{3}\right) \delta 8.69-8.67(\mathrm{~m}, 1 \mathrm{H}), 8.12(\mathrm{~d}, J=2.0 \mathrm{~Hz}, 1 \mathrm{H}), 7.81(\mathrm{dd}, J=8.5$, $2.0 \mathrm{~Hz}, 1 \mathrm{H}), 7.77-7.74(\mathrm{~m}, 1 \mathrm{H}), 7.69-7.67(\mathrm{~m}, 1 \mathrm{H}), 7.52(\mathrm{~d}, J=8.5 \mathrm{~Hz}, 1 \mathrm{H}), 7.28-7.25(\mathrm{~m}, 1 \mathrm{H}) .{ }^{13} \mathbf{C ~ N M R}$ $\left(101 \mathrm{MHz} \mathrm{CDCl}_{3}\right) \delta 154.8,149.8,139.2,137.0,133.1,133.0,130.6,128.8,125.9,122.8,120.3$. Analytic data match literature ${ }^{16}$.

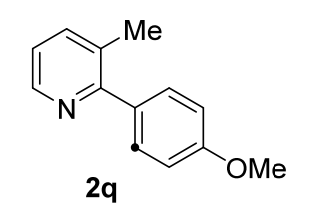

(1) $80 \% \quad 281 \% \quad 371 \%$

All three conditions were run with $0.1 \mathrm{mmol} \mathbf{1 q}$. 2q: Colorless oil. ${ }^{1} \mathbf{H} \mathbf{N M R}\left(500 \mathrm{MHz}, \mathrm{CDCl}_{3}\right) \delta 8.50(\mathrm{~d}, J=$ $4.0 \mathrm{~Hz}, 1 \mathrm{H}), 7.54(\mathrm{~d}, J=7.5 \mathrm{~Hz}, 1 \mathrm{H}), 7.48(\mathrm{~d}, J=9.0 \mathrm{~Hz}, 2 \mathrm{H}), 7.13(\mathrm{dd}, J=8.0,5.0 \mathrm{~Hz}, 1 \mathrm{H}), 6.97(\mathrm{~d}, J=9.0$ $\mathrm{Hz}, 2 \mathrm{H}), 3.85$ (s, 3H), 2.36 (s, 3H). ${ }^{13} \mathbf{C} \mathbf{N M R}\left(125 \mathrm{MHz}, \mathrm{CDCl}_{3}\right) \delta$ 159.3, 158.2, 146.8, 138.4, 133.1, 130.6, 130.2, 121.6, 113.4, 55.2, 20.2. Analytic data match literature ${ }^{17}$.

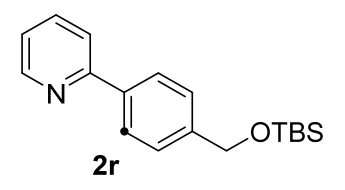

(1) $50 \% \quad 270 \% \quad 3 \quad 34 \%$

All three conditions were run with 0.1 mmol 1r. 2 r: White solid. ${ }^{1} \mathbf{H}$ NMR $\left(500 \mathrm{MHz}, \mathrm{CDCl}_{3}\right) \delta 8.69-8.68(\mathrm{~m}$, 1H), $7.98-7.96(\mathrm{~m}, 2 \mathrm{H}), 7.75-7.71(\mathrm{~m}, 2 \mathrm{H}), 7.44(\mathrm{~d}, J=8.5 \mathrm{~Hz}, 2 \mathrm{H}), 7.22-7.20(\mathrm{~m}, 1 \mathrm{H}), 4.81(\mathrm{~s}, 2 \mathrm{H}), 0.96$ $(\mathrm{s}, 9 \mathrm{H}), 0.12(\mathrm{~s}, 6 \mathrm{H}) .{ }^{13} \mathbf{C}$ NMR $\left(101 \mathrm{MHz}, \mathrm{CDCl}_{3}\right) \delta 157.4,149.6,142.3,138.0,136.7,126.7,126.4,121.9$, $120.4,64.7,25.9,18.4,-5.2$. Analytic data match literature ${ }^{18}$. 

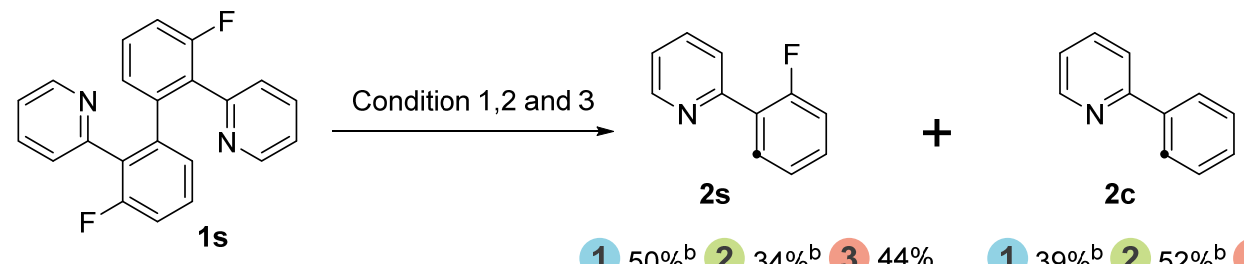

$150 \%^{\mathrm{b}} 234 \%^{\mathrm{b}} 344 \% \quad 139 \%^{\mathrm{b}} 252 \%^{\mathrm{b}} 314 \%$

All three conditions were run with $0.2 \mathrm{mmol} \mathbf{1 s .} \mathbf{2 s}$ and $\mathbf{2 c}$ are inseparable, mixture is obtained as faint yellow oil. Mixture of 2s and 2c (50/39). ${ }^{13} \mathbf{C}$ NMR $\left(101 \mathrm{MHz}, \mathrm{CDCl}_{3}\right) \delta 160.0(\mathrm{~d}, J=250.7 \mathrm{~Hz}), 153.3(\mathrm{~d}, J=1.9 \mathrm{~Hz})$, 149.7, 136.2, 130.9 (d, $J=3.1 \mathrm{~Hz}), 130.3$ (d, $J=8.6 \mathrm{~Hz}), 127.4(\mathrm{~d}, J=11.6 \mathrm{~Hz}), 124.5(\mathrm{~d}, J=3.4 \mathrm{~Hz}), 124.4(\mathrm{~d}$, $J=1.1 \mathrm{~Hz}), 122.3,116.0(\mathrm{~d}, J=22.8 \mathrm{~Hz}) .{ }^{\mathbf{1 9}} \mathbf{F} \mathbf{N M R}\left(470 \mathrm{MHz}, \mathrm{CDCl}_{3}\right) \delta-117.4 .{ }^{\mathbf{1 3}} \mathbf{C} \mathbf{N M R}$ and ${ }^{\mathbf{1 9}} \mathbf{F}$ NMR data match the literature ${ }^{19}$.
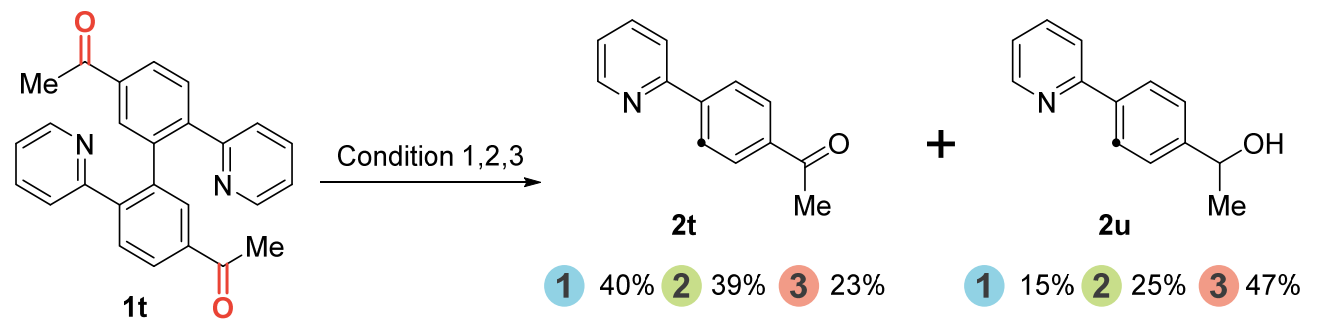

All three conditions were run with $0.2 \mathrm{mmol} \mathbf{1 t}$. Both $\mathbf{2 t}$ and $\mathbf{2 u}$ was observed after reaction. $2 \mathbf{t}$ : White solid. $\mathbf{m}$. p. $117-118{ }^{\circ} \mathrm{C}:{ }^{1} \mathbf{H}$ NMR $\left(400 \mathrm{MHz}, \mathrm{CDCl}_{3}\right) \delta 8.73(\mathrm{dd}, J=4.8,1.2 \mathrm{~Hz}, 1 \mathrm{H}), 8.08(\mathrm{q}, J=8.3 \mathrm{~Hz}, 4 \mathrm{H}), 7.82-$ $7.77(\mathrm{~m}, 2 \mathrm{H}), 7.33-7.27(\mathrm{~m}, 1 \mathrm{H}), 2.65(\mathrm{dd}, J=3.3,0.8 \mathrm{~Hz}, 3 \mathrm{H}) .{ }^{13} \mathbf{C} \mathbf{N M R}\left(101 \mathrm{MHz}, \mathrm{CDCl}_{3}\right) \delta 197.8,156.1$, 149.9, 143.5, 137.1, 137.0, 128.8, 127.0, 122.9, 121.0, 26.8. HRMS calcd. for $\mathrm{C}_{13} \mathrm{H}_{12} \mathrm{NO}^{+}[\mathrm{M}+\mathrm{H}]^{+}: 198.0913$, found: 198.0918. IR $\left(\mathrm{cm}^{-1}\right)$ 1680, 1399, 1356, 849, 783, 723, 592.
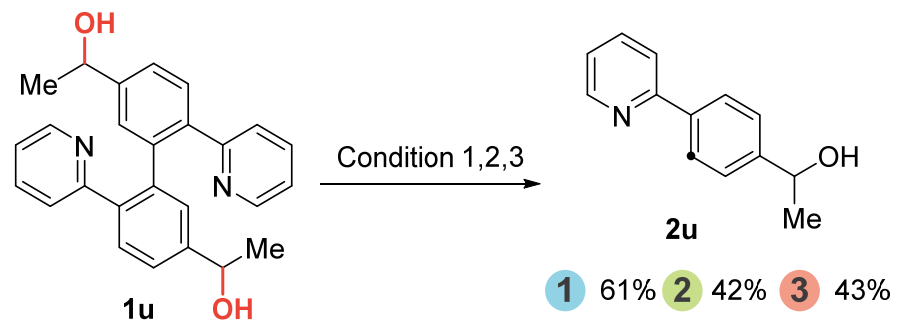

All three conditions were run with $0.2 \mathrm{mmol} 1 \mathbf{1 u} .{ }^{1} \mathbf{H}$ NMR $\left(400 \mathrm{MHz}, \mathrm{CDCl}_{3}\right) \delta 8.69-8.66(\mathrm{~m}, 1 \mathrm{H}), 7.98-$ $7.91(\mathrm{~m}, 2 \mathrm{H}), 7.78-7.68(\mathrm{~m}, 2 \mathrm{H}), 7.49-7.43(\mathrm{~m}, 2 \mathrm{H}), 7.25-7.19(\mathrm{~m}, 1 \mathrm{H}), 4.95(\mathrm{q}, J=6.1 \mathrm{~Hz}, 1 \mathrm{H}), 2.30(\mathrm{~s}$, 1H), $1.52(\mathrm{dd}, J=6.5,1.1 \mathrm{~Hz}, 3 \mathrm{H}) .{ }^{13} \mathbf{C}$ NMR (101 MHz, $\left.\mathrm{CDCl}_{3}\right) \delta 157.1,149.6,146.7,138.4,136.8,127.1$, 125.8, 122.1, 120.5, 70.1, 25.2. HRMS calcd. for $\mathrm{C}_{13} \mathrm{H}_{14} \mathrm{NO}^{+}[\mathrm{M}+\mathrm{H}]^{+}:$200.1070, found: 200.1074. IR (cm $\left.{ }^{-1}\right)$ 3266, 2971, 2866, 1591, 1473, 1436, 1403, 1288, 1120, 1061, 1014.

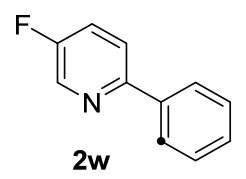

(1) $67 \% \quad 2 \quad 71 \% \quad 3 \quad 63 \%$ 
Condition 1: $0.1 \mathrm{mmol} \mathrm{1w}$, 3h; condition 2: $0.1 \mathrm{mmol} \mathrm{1w}$; condition 3: $0.2 \mathrm{mmol} \mathrm{1w.} \mathrm{2w}$ : Pale yellow solid. ${ }^{1} \mathrm{H}$ $\operatorname{NMR}\left(400 \mathrm{MHz}, \mathrm{CDCl}_{3}\right) \delta 8.55(\mathrm{~d}, J=2.8 \mathrm{~Hz}, 1 \mathrm{H}), 7.96-7.93(\mathrm{~m}, 2 \mathrm{H}), 7.73-7.69(\mathrm{~m}, 1 \mathrm{H}), 7.50-7.39(\mathrm{~m}$, 4H). ${ }^{13}$ C NMR (125 MHz, $\left.\mathrm{CDCl}_{3}\right) \delta 158.8(\mathrm{~d}, J=318.4 \mathrm{~Hz}), 153.7(\mathrm{~d}, J=4.8 \mathrm{~Hz}), 138.4,137.6(\mathrm{~d}, J=29.2$ $\mathrm{Hz}), 129.8,128.8,126.7,123.4(\mathrm{~d}, \mathrm{~J}=22.9 \mathrm{~Hz}), 121.2(\mathrm{~d}, J=5.4 \mathrm{~Hz}) .{ }^{19} \mathbf{F} \mathbf{N M R}\left(470 \mathrm{MHz}, \mathrm{CDCl}_{3}\right) \delta-129.8$. HRMS calcd. for $\mathrm{C}_{11} \mathrm{H}_{9} \mathrm{FN}^{+}[\mathrm{M}+\mathrm{H}]^{+}:$174.0714, found: 174.0717. IR $\left(\mathrm{cm}^{-1}\right) 3094,3058,3026,1589,1574$, $1501,1473,1447,1378,1297,1238,1225,1123,1072,1012$.

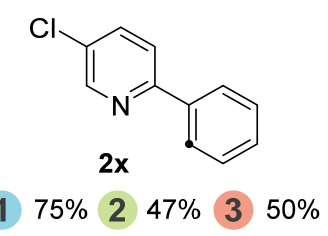

Condition 1: $0.1 \mathrm{mmol} \mathrm{1x}$, 3h; condition 2: $0.2 \mathrm{mmol} \mathrm{1x}$; condition 3: $0.2 \mathrm{mmol} \mathrm{1x}$. 2x: White solid. ${ }^{\mathbf{1}} \mathbf{H}$ NMR $\left(500 \mathrm{MHz}, \mathrm{CDCl}_{3}\right) \delta 8.64(\mathrm{~d}, J=1.5 \mathrm{~Hz}, 1 \mathrm{H}), 7.97-7.95(\mathrm{~m}, 2 \mathrm{H}), 7.71(\mathrm{dd}, J=8.0,2.5 \mathrm{~Hz}, 1 \mathrm{H}), 7.67(\mathrm{dd}, J=$ 8.5, 0.5 Hz, 1H), $7.49-7.44(\mathrm{~m}, 2 \mathrm{H}), 7.44-7.42(\mathrm{~m}, 1 \mathrm{H}) .{ }^{13} \mathbf{C} \mathbf{N M R}\left(125 \mathrm{MHz}, \mathrm{CDCl}_{3}\right) \delta 155.5,148.4,138.1$, $136.4,130.5,129.2,128.8,126.7,121.1$. Analytic data match literature ${ }^{20}$.

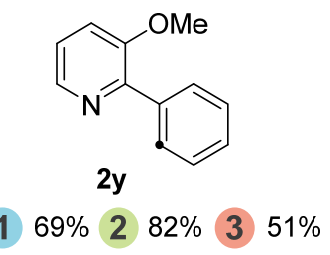

Condition 1: $0.1 \mathrm{mmol} \mathrm{1y}, 3 \mathrm{~h}$; condition 2: $0.2 \mathrm{mmol} \mathrm{1y}$; condition 3: $0.2 \mathrm{mmol} \mathrm{1y}$. $2 \mathbf{y}$ : Colorless oil. ${ }^{\mathbf{1}} \mathbf{H}$ NMR $\left(500 \mathrm{MHz}, \mathrm{CDCl}_{3}\right) \delta 8.35(\mathrm{dd}, J=4.5,1.5 \mathrm{~Hz}, 1 \mathrm{H}), 7.92-7.89(\mathrm{~m}, 2 \mathrm{H}), 7.46-7.43(\mathrm{~m}, 2 \mathrm{H}), 7.40-7.37(\mathrm{~m}$, 1H), $7.28(\mathrm{dd}, J=8.0,1.0 \mathrm{~Hz}, 1 \mathrm{H}), 7.24-7.22(\mathrm{~m}, 1 \mathrm{H}), 3.85(\mathrm{~s}, 3 \mathrm{H}) .{ }^{13} \mathbf{C} \mathbf{N M R}\left(125 \mathrm{MHz}, \mathrm{CDCl}_{3}\right) \delta 153.5$, $148.1,141.3,137.6,129.3,128.2,127.9,122.8,118.4,55.4$. Analytic data match literature ${ }^{21}$.

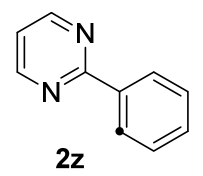

(1) $22 \% \quad 2 \quad 34 \% \quad 3 \quad 40 \%$

Pale yellow solid. ${ }^{1} \mathbf{H}$ NMR $\left(400 \mathrm{MHz}, \mathrm{CDCl}_{3}\right) \delta 8.80(\mathrm{dd}, J=4.8,1.8 \mathrm{~Hz}, 2 \mathrm{H}), 8.45-8.39(\mathrm{~m}, 2 \mathrm{H}), 7.50-$ $7.46(\mathrm{~m}, 3 \mathrm{H}), 7.18(\mathrm{td}, J=4.8,2.7 \mathrm{~Hz}, 1 \mathrm{H}) .{ }^{13} \mathrm{C} \mathbf{N M R}\left(101 \mathrm{MHz}, \mathrm{CDCl}_{3}\right) \delta 164.7,157.2,137.5,130.7,128.6$, 128.1, 119.0. HRMS calcd. for $\mathrm{C}_{10} \mathrm{H}_{9} \mathrm{~N}_{2}^{+}[\mathrm{M}+\mathrm{H}]^{+}:$157.0760, found: 157.0760. IR $\left(\mathrm{cm}^{-1}\right)$ 1567, 1555, 1418, $1093,1026,746,693,643$. Analytic data match the literature ${ }^{22}$. 


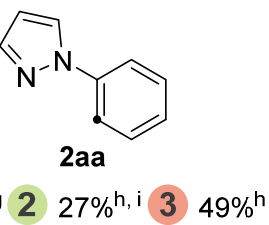

Condition 1: $0.2 \mathrm{mmol} \mathrm{1aa,} 20 \mathrm{~mol} \% \mathrm{Ru}(\mathrm{COD}) \mathrm{Cl}_{2}, 160{ }^{\circ} \mathrm{C}$; condition 2: $0.1 \mathrm{mmol} \mathbf{1 a a}, 20 \mathrm{~mol} \% \mathrm{Ru}(\mathrm{COD}) \mathrm{Cl}_{2}$, $150{ }^{\circ} \mathrm{C}$; condition 3: $0.1 \mathrm{mmol}$ 1aa, $20 \mathrm{~mol} \% \mathrm{Ru}(\mathrm{COD}) \mathrm{Cl}_{2}, 150{ }^{\circ} \mathrm{C}$. 2aa: Colorless oil. ${ }^{1} \mathbf{H}$ NMR $(400 \mathrm{MHz}$, $\left.\mathrm{CDCl}_{3}\right) \delta 7.93(\mathrm{dd}, J=2.4,0.4 \mathrm{~Hz}, 1 \mathrm{H}), 7.76-7.66(\mathrm{~m}, 3 \mathrm{H}), 7.50-7.41(\mathrm{~m}, 2 \mathrm{H}), 7.32-7.26(\mathrm{~m}, 1 \mathrm{H}), 6.47(\mathrm{dt}$, $J=8.1,4.0 \mathrm{~Hz}, 1 \mathrm{H}) .{ }^{13} \mathbf{C}$ NMR $\left(101 \mathrm{MHz}, \mathrm{CDCl}_{3}\right) \delta 141.0,140.2,129.4,126.7,126.4,119.2,107.6$. Analytic data match the literature ${ }^{23}$.

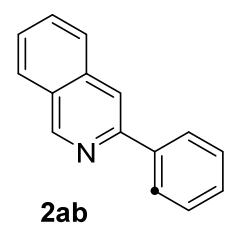

(1) $49 \% \quad 2 \quad 84 \% \quad 373 \%$

All three conditions were run with 0.2 mmol 1ab. 2ab: White solid. m. p.188-189 ${ }^{\circ} \mathrm{C} .{ }^{1} \mathbf{H}$ NMR $(400 \mathrm{MHz}$, $\left.\mathrm{CDCl}_{3}\right) \delta 9.35(\mathrm{~s}, 1 \mathrm{H}), 8.16-8.11(\mathrm{~m}, 2 \mathrm{H}), 8.07(\mathrm{~s}, 1 \mathrm{H}), 7.99(\mathrm{dd}, J=8.2,0.9 \mathrm{~Hz}, 1 \mathrm{H}), 7.87(\mathrm{dd}, J=8.2,0.7$ Hz, 1H), 7.69 (ddd, $J=8.2,6.9,1.3 \mathrm{~Hz}, 1 \mathrm{H}), 7.58$ (ddd, $J=8.1,6.9,1.2 \mathrm{~Hz}, 1 \mathrm{H}), 7.55-7.49$ (m, 2H), $7.45-$ $7.40(\mathrm{~m}, 1 \mathrm{H}) .{ }^{13} \mathrm{C}$ NMR $\left(101 \mathrm{MHz}, \mathrm{CDCl}_{3}\right) \delta 152.4,151.2,139.6,136.6,130.5,128.8,128.5,127.7,127.5$, 127.0, 127.0, 126.9, 116.5. HRMS calcd. for $\mathrm{C}_{15} \mathrm{H}_{12} \mathrm{~N}^{+}[\mathrm{M}+\mathrm{H}]^{+}: 206.0964$, found: 206.0971. IR $\left(\mathrm{cm}^{-1}\right)$ 2923, 1624, 1453, 1331, 1281, 1221, 962, 884, 786, 762, 688, 682. 


\section{One-pot functionalization}

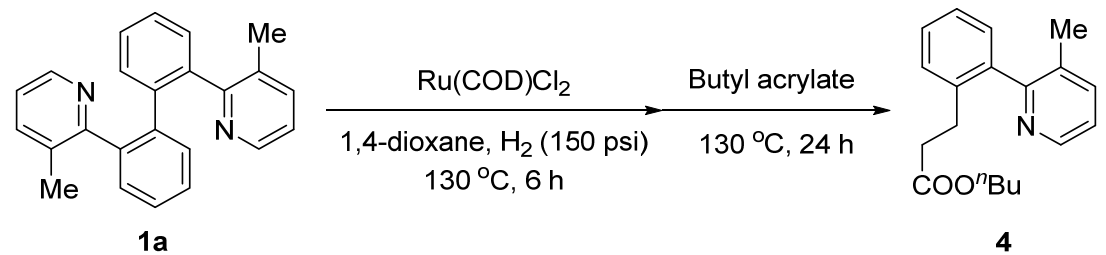

To a Q-tube charged with a stir bar, substrate (1a, $67.3 \mathrm{mg}, 0.2 \mathrm{mmol})$ and $\mathrm{Ru}(\mathrm{COD}) \mathrm{Cl}_{2}(5.6 \mathrm{mg}, 0.02$ mmol) were added. The Q-tube was then transferred into the glovebox. Distilled 1,4-dioxane (2.6 mL) was added. The Q-tube was reassembled and transferred out of the glovebox. The reaction mixture was in situ freeze-pump-thawed using standard technique for three times. The Q-tube was then charged with 150 psi hydrogen gas followed by heating at $130{ }^{\circ} \mathrm{C}$ in a pre-heated oil bath for $6 \mathrm{~h}$. Upon completion of the reaction, the reaction mixture was transferred into the glovebox, the pressure of $\mathrm{H}_{2}$ was released, butyl acrylate (128.2 mg, $1.0 \mathrm{mmol}$ ) was added to the reaction mixture. The Q-tube was reassembled and transferred out of the glovebox followed by heating to $130{ }^{\circ} \mathrm{C}$ for $24 \mathrm{~h}$. Upon completion of the reaction, the reaction mixture was concentrated under reduced pressure and directly purified using column chromatography on silica gel to afford the desired product as a pale yellow oil (96.2 $\mathrm{mg}, 81 \%$ yield). ${ }^{1} \mathbf{H}$ NMR $\left(500 \mathrm{MHz}, \mathrm{CDCl}_{3}\right) \delta 8.51(\mathrm{~d}, J=4.0$ $\mathrm{Hz}, 1 \mathrm{H}), 7.60(\mathrm{~d}, J=7.6 \mathrm{~Hz}, 1 \mathrm{H}), 7.37-7.25(\mathrm{~m}, 3 \mathrm{H}), 7.21(\mathrm{dd}, J=7.7,4.8 \mathrm{~Hz}, 1 \mathrm{H}), 7.17(\mathrm{~d}, J=7.4 \mathrm{~Hz}, 1 \mathrm{H})$, $4.00(\mathrm{t}, J=6.7 \mathrm{~Hz}, 2 \mathrm{H}), 2.85-2.68(\mathrm{~m}, 2 \mathrm{H}), 2.45(\mathrm{t}, J=8.0 \mathrm{~Hz}, 2 \mathrm{H}), 2.13(\mathrm{~s}, 3 \mathrm{H}), 1.59-1.48(\mathrm{~m}, 2 \mathrm{H}), 1.37-$ $1.24(\mathrm{~m}, 2 \mathrm{H}), 0.90(\mathrm{t}, J=7.4 \mathrm{~Hz}, 3 \mathrm{H}) .{ }^{13} \mathbf{C} \mathbf{N M R}\left(126 \mathrm{MHz}, \mathrm{CDCl}_{3}\right) \delta$ 173.0, 159.0, 146.6, 140.0, 138.2, 137.9, $131.5,129.2,128.9,128.1,126.2,122.3,64.1,35.1,30.5,28.3,19.2,19.0,13.6$. HRMS APCI (m/z): [M+H] ${ }^{+}$ calcd for $\mathrm{C}_{19} \mathrm{H}_{24} \mathrm{NO}_{2}{ }^{+}, 298.1802$; found, 298.1820. IR (cm $\left.{ }^{-1}\right)$ 3058, 2959, 2873, 1732, 1584, 1567, 1492, 1447, $1423,1383,1360,1290,1180,1120,1064,1025$. 


\section{Mechanistic study}

\subsection{The fate of 1,5-cyclooctadiene (COD)}

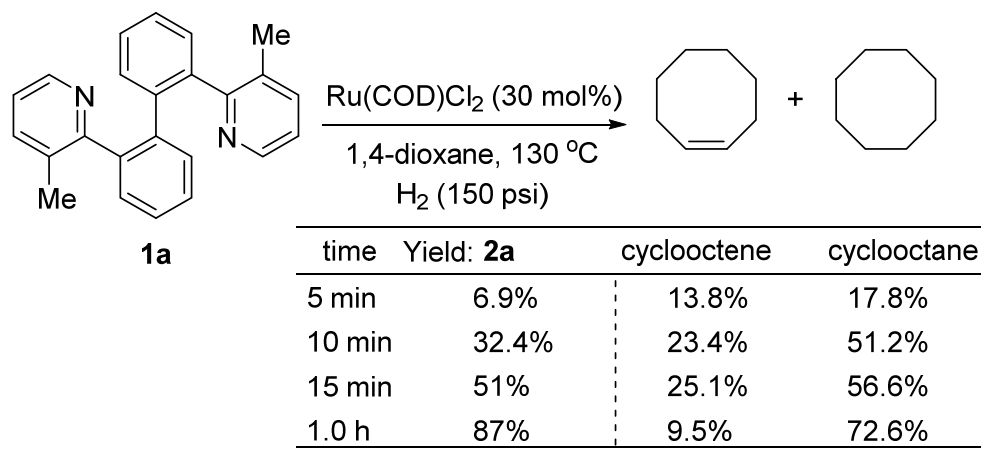

To a Q-tube charged with a stir bar, standard substrate 1a $(67.3 \mathrm{mg}, 0.2 \mathrm{mmol})$ and $\mathrm{Ru}(\mathrm{COD}) \mathrm{Cl}_{2}(16.8 \mathrm{mg}$, $0.06 \mathrm{mmol})$ were added. The Q-tube was then transferred into the glovebox. Distilled 1,4-dioxane $(2.66 \mathrm{~mL})$ was added. The Q-tube was reassembled, transferred out of the glovebox and flushed with hydrogen gas for 10 times then charged with 150 psi hydrogen gas followed by heating at $130{ }^{\circ} \mathrm{C}$ in a pre-heated oil bath. The reaction was stopped at indicated time points by releasing the hydrogen pressure and chilling the tube in an acetone-dry ice bath. The yield of cyclooctene and cyclooctane was determined by GC-MS using dodecane as internal standard. The yield of 2a was determined by ${ }^{1} \mathrm{H}-\mathrm{NMR}$ using 1,1,2,2-tetrachloroethane as internal standard. This experiment indicated that the COD was hydrogenated at the beginning of the reaction.

\subsection{Kinetic study}

\subsubsection{Initial rates vs $\left[\mathrm{Ru}(\mathrm{COD}) \mathrm{Cl}_{2}\right]$}

To a Q-tube charged with a stir bar, standard substrate 1a $(67.3 \mathrm{mg}, 0.2 \mathrm{mmol})$ and $\mathrm{Ru}(\mathrm{COD}) \mathrm{Cl}_{2}(1.2 \mathrm{mg}$ (2.0 mol\%), $2.0 \mathrm{mg}(3.5 \mathrm{~mol} \%), 2.8 \mathrm{mg}(5.0 \mathrm{~mol} \%)$ and $3.6 \mathrm{mg}(6.5 \mathrm{~mol} \%))$ were added respectively. The Q-tube was then transferred into the glovebox. Distilled 1,4-dioxane $(2.66 \mathrm{~mL})$ was added. The Q-tube was reassembled, transferred out of the glovebox and flushed with hydrogen gas for 10 times then charged with 150 psi hydrogen gas followed by heating at $130{ }^{\circ} \mathrm{C}$ in a pre-heated oil bath. The reaction was stopped at indicated time points by releasing the hydrogen pressure and chilling the tube in an acetone-dry ice bath. The yield of the product was determined by GC-FID using tetradecane as internal standard. First order on $\left[\mathrm{Ru}(\mathrm{COD}) \mathrm{Cl}_{2}\right]$ was observed.

Figure S5.2.1.1 initial rates vs $\left[\mathrm{Ru}(\mathrm{COD}) \mathrm{Cl}_{2}\right](1.2 \mathrm{mg}, 2.0 \mathrm{~mol} \%)$

\begin{tabular}{|c|c|c|c|}
\hline $\mathrm{Ru}(\mathrm{COD}) \mathrm{Cl}_{2}(\mathrm{M})$ & time $(\mathrm{min})$ & $\mathbf{2 a}(\mathrm{M})$ & Initial rate $(\mathrm{M} / \mathrm{min})$ \\
\hline \multirow{3}{*}{0.00161} & 50 & 0.011274 & \multirow{3}{*}{0.000156} \\
\cline { 2 - 3 } & 70 & 0.015069 & \\
\cline { 2 - 3 } & 90 & 0.017376 & \\
\cline { 2 - 3 } & 110 & 0.020921 & \\
\hline
\end{tabular}




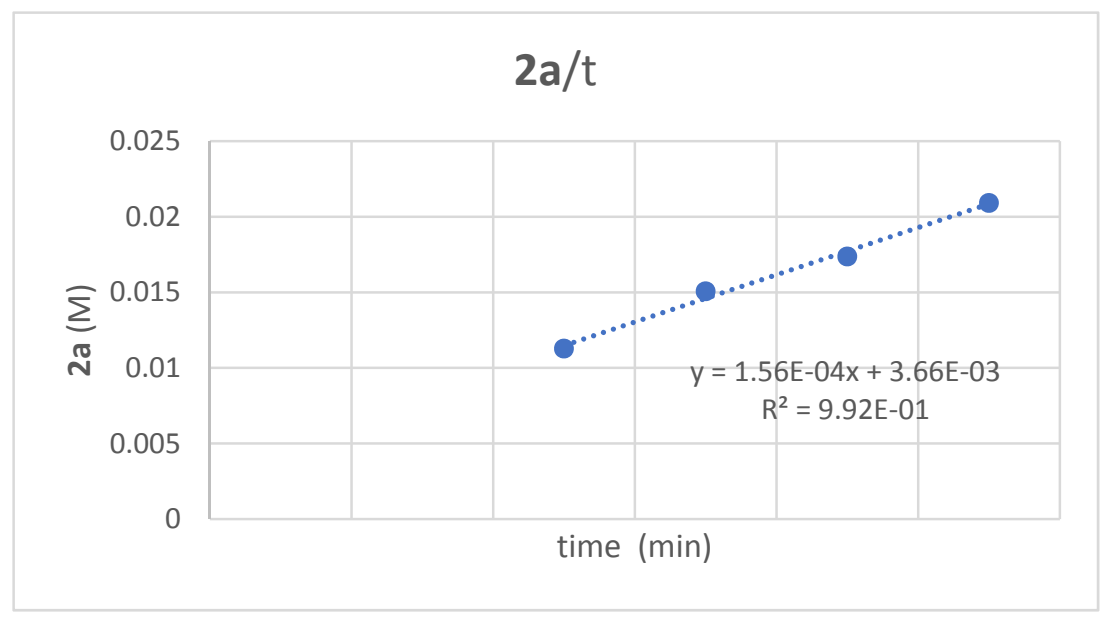

Figure S5.2.1.2 initial rates vs $\left[\mathrm{Ru}(\mathrm{COD}) \mathrm{Cl}_{2}\right](2.0 \mathrm{mg}, 3.5 \mathrm{~mol} \%)$

\begin{tabular}{|c|c|c|c|}
\hline $\mathrm{Ru}(\mathrm{COD}) \mathrm{Cl}_{2}(\mathrm{M})$ & time $(\mathrm{min})$ & $\mathbf{2 a}(\mathrm{M})$ & Initial rate $(\mathrm{M} / \mathrm{min})$ \\
\hline \multirow{3}{*}{0.002684} & 20 & 0.011513 & \multirow{2}{*}{0.000263} \\
\cline { 2 - 3 } & 30 & 0.015453 & \multirow{2}{*}{0.0017961} \\
\cline { 2 - 3 } & 40 & 0.0179 & \\
\cline { 2 - 3 } & 50 & 0.019082 & \\
\cline { 2 - 3 } & 60 & 0.022852 & \\
\hline
\end{tabular}

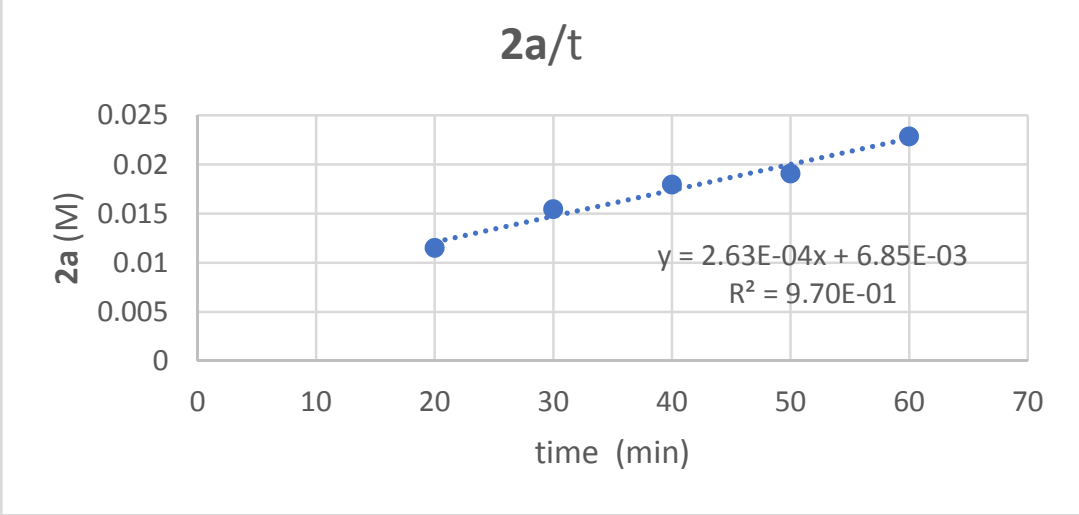

Figure S5.2.1.3 initial rates vs $\left[\mathrm{Ru}(\mathrm{COD}) \mathrm{Cl}_{2}\right](2.8 \mathrm{mg}, 5.0 \mathrm{~mol} \%)$

\begin{tabular}{|c|c|c|c|}
\hline $\mathrm{Ru}(\mathrm{COD}) \mathrm{Cl}_{2}(\mathrm{M})$ & time $(\mathrm{min})$ & $\mathbf{2 a}(\mathrm{M})$ & Initial rate $(\mathrm{M} / \mathrm{min})$ \\
\hline \multirow{4}{*}{0.003757} & 10 & 0.010713 & \\
\cline { 2 - 3 } & 20 & 0.013683 & \multirow{2}{*}{0.000432} \\
\cline { 2 - 3 } & 30 & 0.01671 & \\
\cline { 2 - 3 } & 40 & 0.023323 & \\
\cline { 2 - 3 } & 50 & 0.027507 & \\
\hline
\end{tabular}




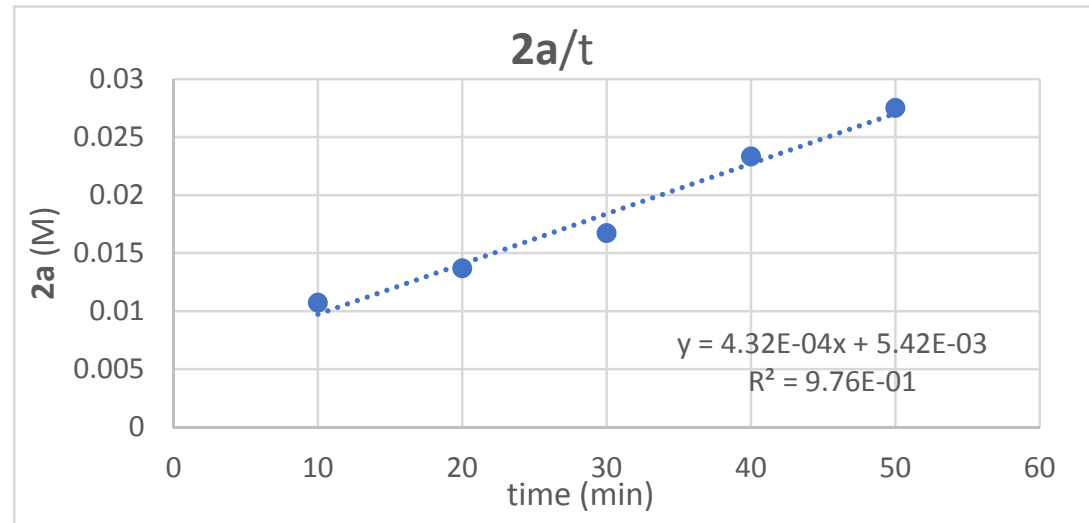

Figure S5.2.1.4 initial rates vs $\left[\mathrm{Ru}(\mathrm{COD}) \mathrm{Cl}_{2}\right](3.6 \mathrm{mg}, 6.5 \mathrm{~mol} \%)$

\begin{tabular}{|c|c|c|c|}
\hline $\mathrm{Ru}(\mathrm{COD}) \mathrm{Cl}_{2}(\mathrm{M})$ & time $(\mathrm{min})$ & $\mathbf{2 a}(\mathrm{M})$ & Initial rate $(\mathrm{M} / \mathrm{min})$ \\
\hline \multirow{3}{*}{0.004831} & 15 & 0.016514 & \\
\cline { 2 - 3 } & 25 & 0.022104 & \multirow{2}{*}{0.000554} \\
\cline { 2 - 3 } & 40 & 0.030715 & \\
\cline { 2 - 3 } & 30 & 0.025262 & \\
\cline { 2 - 3 } & 35 & 0.026891 & \\
\hline
\end{tabular}

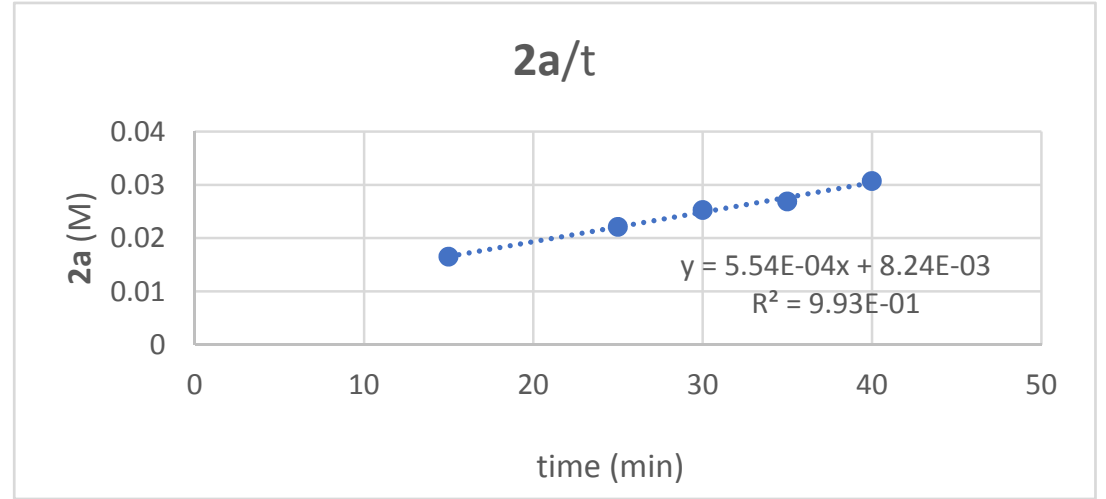

Table S5.2.1. Order for $\left[\mathrm{Ru}(\operatorname{cod}) \mathrm{Cl}_{2}\right]$

\begin{tabular}{|c|c|}
\hline $\mathrm{C}_{\mathrm{Ru}(\mathrm{COD}) \mathrm{Cl} 2}(\mathrm{M})$ & Initial rate $(\mathrm{M} / \mathrm{min})$ \\
\hline 0.001610 & 0.000156 \\
\hline 0.002684 & 0.000263 \\
\hline 0.003757 & 0.000432 \\
\hline 0.004831 & 0.000554 \\
\hline
\end{tabular}




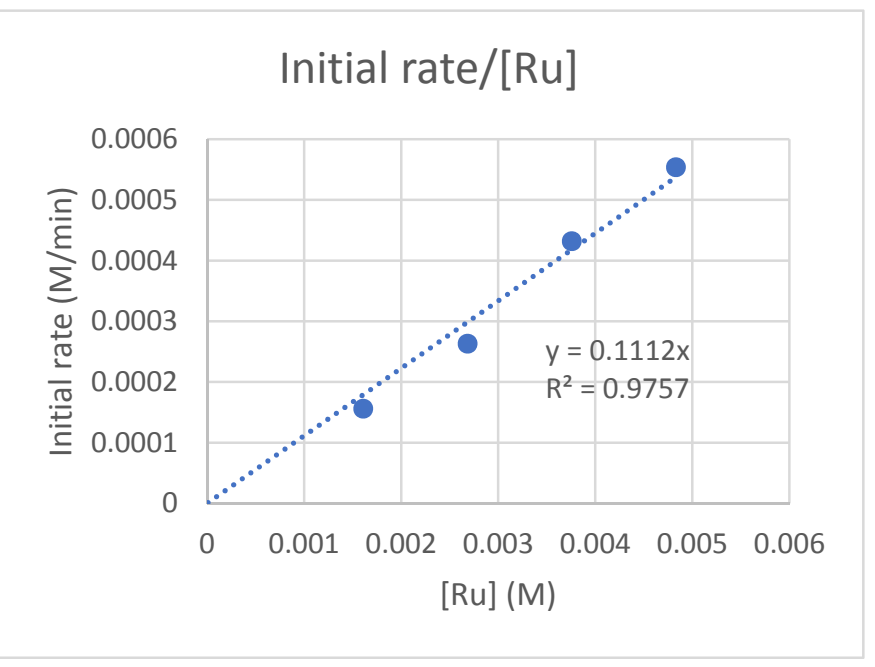

\subsubsection{Initial rates vs [COD]}

To a Q-tube charged with a stir bar, standard substrate $1 \mathrm{a}(67.3 \mathrm{mg}, 0.2 \mathrm{mmol})$ and $\mathrm{Ru}(\mathrm{COD}) \mathrm{Cl}_{2}(2.8 \mathrm{mg})$ were added, The Q-tube was then transferred into the glovebox. Distilled 1,4-dioxane and stork solution of COD (1,5-cyclooctadiene) (433 mg in $10.0 \mathrm{~mL} \mathrm{1,4-dioxane)} \mathrm{were} \mathrm{added} \mathrm{as} \mathrm{the} \mathrm{following} \mathrm{table} \mathrm{indicated.} \mathrm{The}$ Q-tube was reassembled, transferred out of the glovebox and flushed with hydrogen gas for 10 times then charged with $150 \mathrm{psi}$ of hydrogen gas followed by heating at $130{ }^{\circ} \mathrm{C}$ in a pre-heated oil bath. The reaction was stopped at indicated time points by releasing the hydrogen pressure and chilling in an acetone-dry ice bath. The reaction mixture was passed through a Celite pad and flush with DCM, the combined filtration was concentrated in vacuum to afford the crude mixture. To the crude mixture, internal standard (1,1,2,2-tetrachloroethane, $\sim 31 \mathrm{mg})$ was added and the yield of $\mathbf{2 a}$ was determined by ${ }^{1} \mathrm{H}-\mathrm{NMR}$. Zero order on [COD] was observed.

\begin{tabular}{ccc|ccc}
\hline Entry & 1,4-dioxane $(\mathrm{mL})$ & COD solution $(\mathrm{mL})$ & Entry & 1,4-dioxane $(\mathrm{mL})$ & COD solution $(\mathrm{mL})$ \\
\hline 1 & 2.66 & 0 & 3 & 2.46 & 0.2 \\
2 & 2.56 & 0.1 & 4 & 2.36 & 0.3 \\
\hline
\end{tabular}

Figure S5.2.2.1. Initial rates vs [COD] $(0.003759 \mathrm{M})$

\begin{tabular}{|c|c|c|c|}
\hline COD $(\mathrm{M})$ & time $(\mathrm{min})$ & $\mathbf{2 a}(\mathrm{M})$ & Initial rate $(\mathrm{M} / \mathrm{min})$ \\
\hline \multirow{4}{*}{0.003759} & 10 & 0.010713 & \\
\cline { 2 - 3 } & 20 & 0.013683 & \multirow{2}{*}{0.000432} \\
\cline { 2 - 3 } & 30 & 0.01671 & \multirow{2}{*}{0.023323} \\
\cline { 2 - 3 } & 40 & 0.027507 & \\
\cline { 2 - 3 } & 50 & & \\
\hline
\end{tabular}




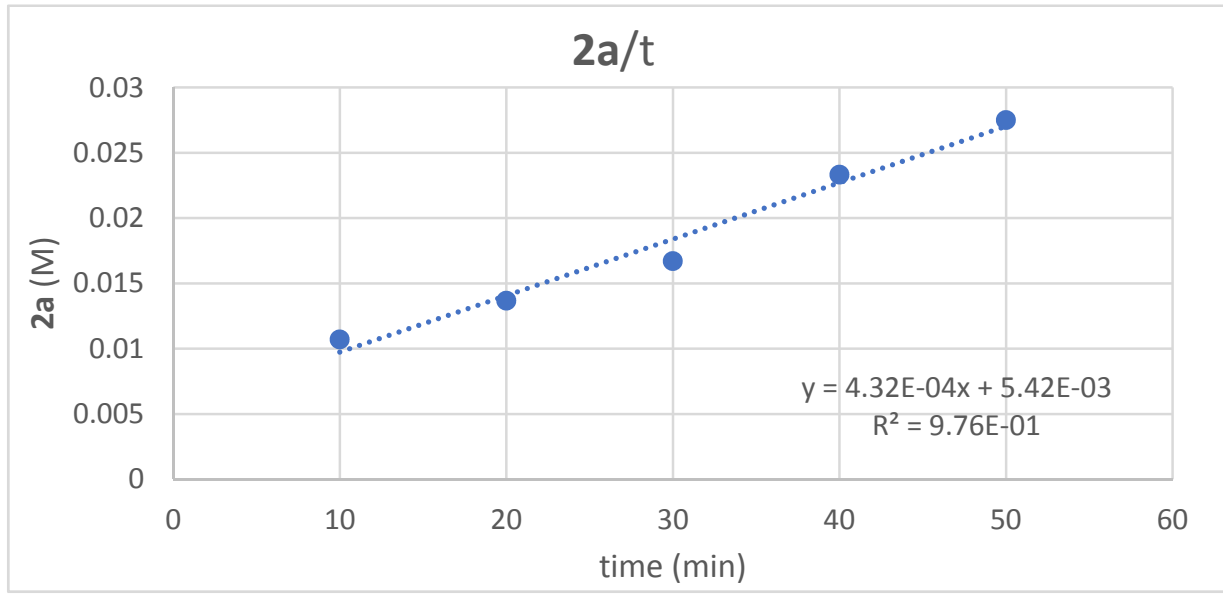

Figure S5.2.2.2 Initial rates vs [COD] $(0.0188 \mathrm{M})$

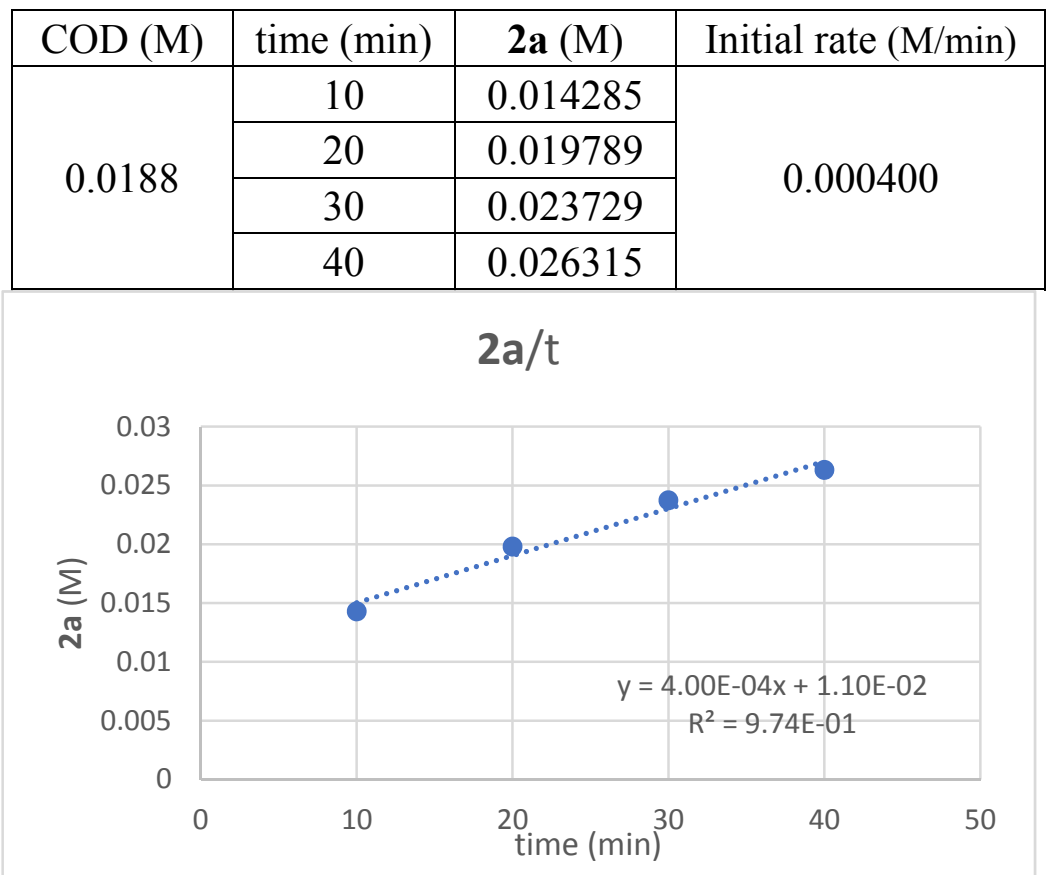

Figure S5.2.2.3 Initial rates vs $[\mathrm{COD}](0.03383 \mathrm{M})$

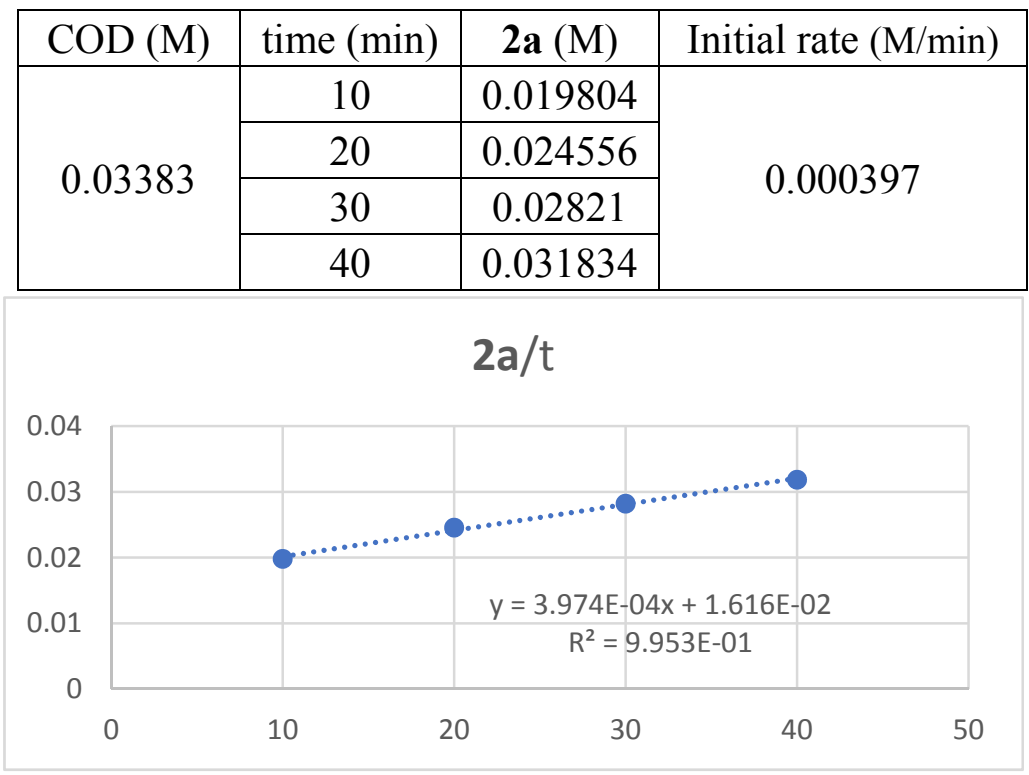

Figure S5.2.2.4 Initial rates vs [COD] $(0.04887 \mathrm{M})$ 


\begin{tabular}{|c|c|c|c|}
\hline COD $(\mathrm{M})$ & time $(\mathrm{min})$ & $\mathbf{2 a}(\mathrm{M})$ & Initial rate $(\mathrm{M} / \mathrm{min})$ \\
\hline \multirow{3}{*}{0.04887} & 10 & 0.018526 & \multirow{2}{*}{0.0004737} \\
\cline { 2 - 3 } & 20 & 0.023217 & \\
\cline { 2 - 3 } & 30 & 0.027954 & \\
\cline { 2 - 3 } & 40 & 0.032736 & \\
\hline
\end{tabular}

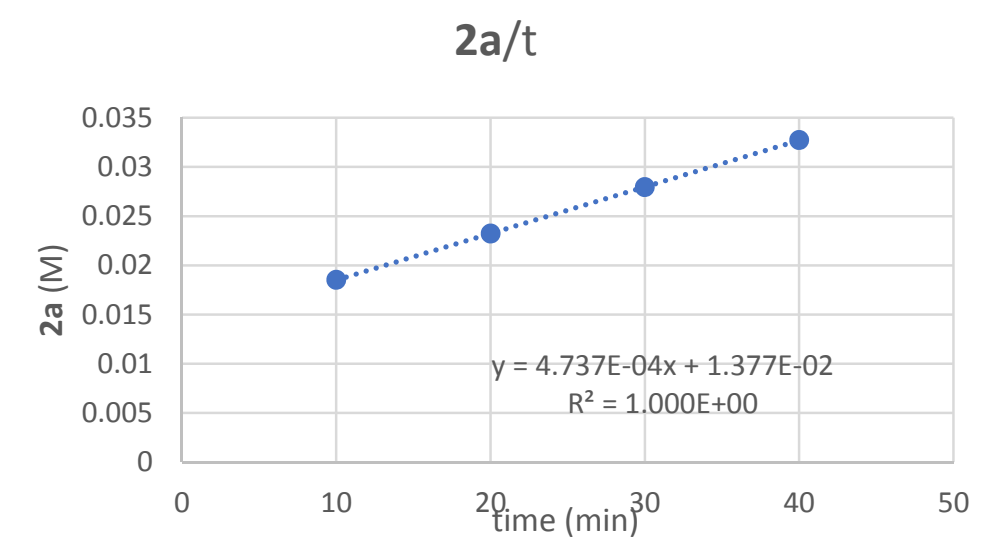

Table S5.2.2 Order for [COD]

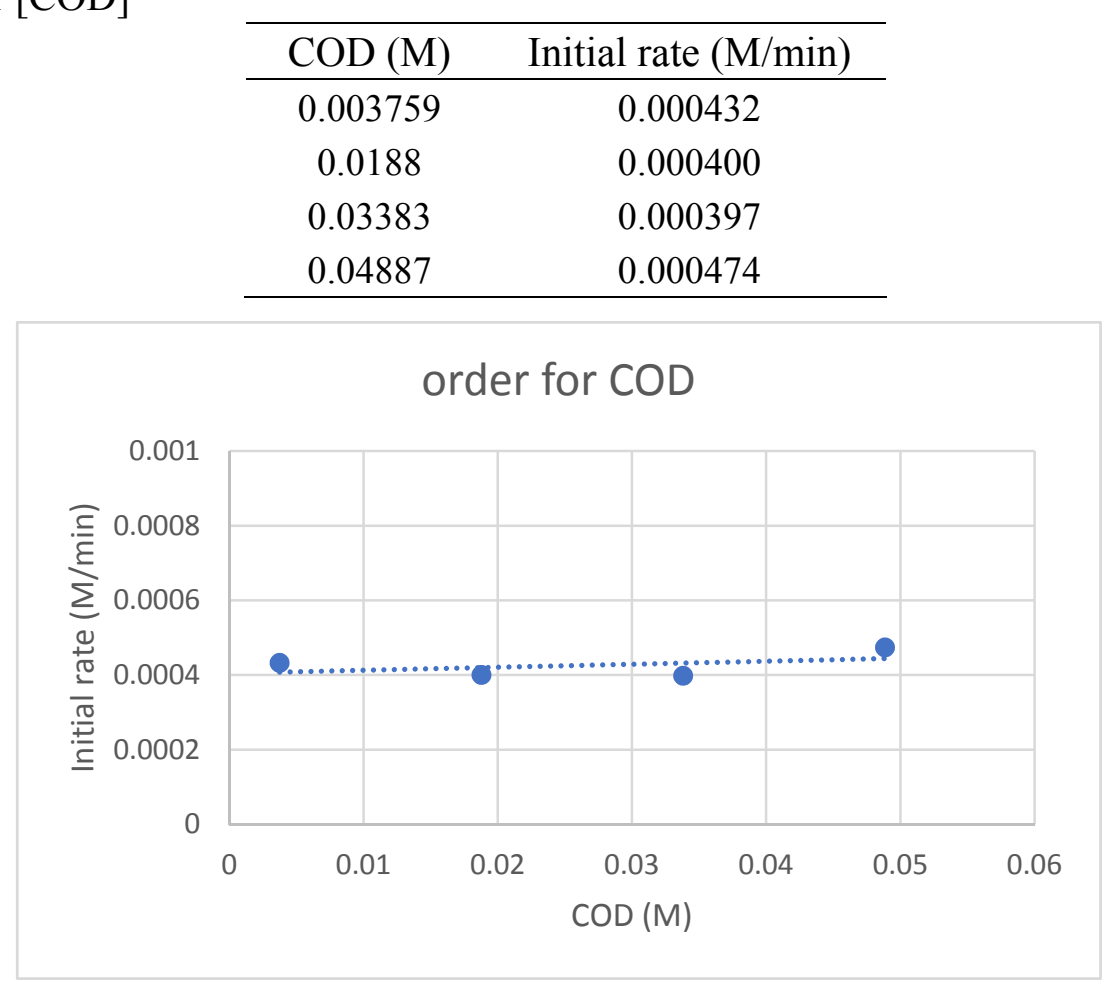

\subsubsection{Initial rates $v s$ the pressure of $\mathrm{H}_{2}$}

To a Q-tube charged with a stir bar, standard substrate $1 \mathbf{a}(67.3 \mathrm{mg}, 0.2 \mathrm{mmol})$ and $\mathrm{Ru}(\mathrm{COD}) \mathrm{Cl}_{2}(2.8 \mathrm{mg})$ were added, The Q-tube was then transferred into the glovebox. Distilled 1,4-dioxane (2.66 mL) was added. The Q-tube was reassembled, transferred out of the glovebox and flushed with hydrogen gas for 10 times then charged with different pressure of hydrogen gas (150 psi, 120 psi, 90 psi, 60 psi, 45 psi, 30 psi, 25 psi, 20 psi) followed by heating at $130{ }^{\circ} \mathrm{C}$ in a pre-heated oil bath. The reaction was stopped at indicated time points by releasing the hydrogen pressure and chilling in an acetone-dry ice bath. The reaction mixture was pass through 
a Celite pad and flush with DCM, the combined filtration was concentrated in vacuum to afford the crude mixture. To the crude mixture, internal standard (1,1,2,2-tetrachloroethane, $\sim 31 \mathrm{mg}$ ) was added and the yield of 1a was determined by ${ }^{1} \mathrm{H}-\mathrm{NMR}$ or GC-FID using tetradecane as internal standard. Zero order dependence on the pressure of hydrogen gas was observed at high pressure ( $>30 \mathrm{psi})$, but some dependence on the pressure of hydrogen gas was observed at low pressure $(20-30$ psi). Due to the technical constraint of Q-tube, the reaction could not be set up below 20 psi of hydrogen gas.

Figure 5.2.3.1. Initial rates vs $\mathrm{H}_{2}(150 \mathrm{psi})$

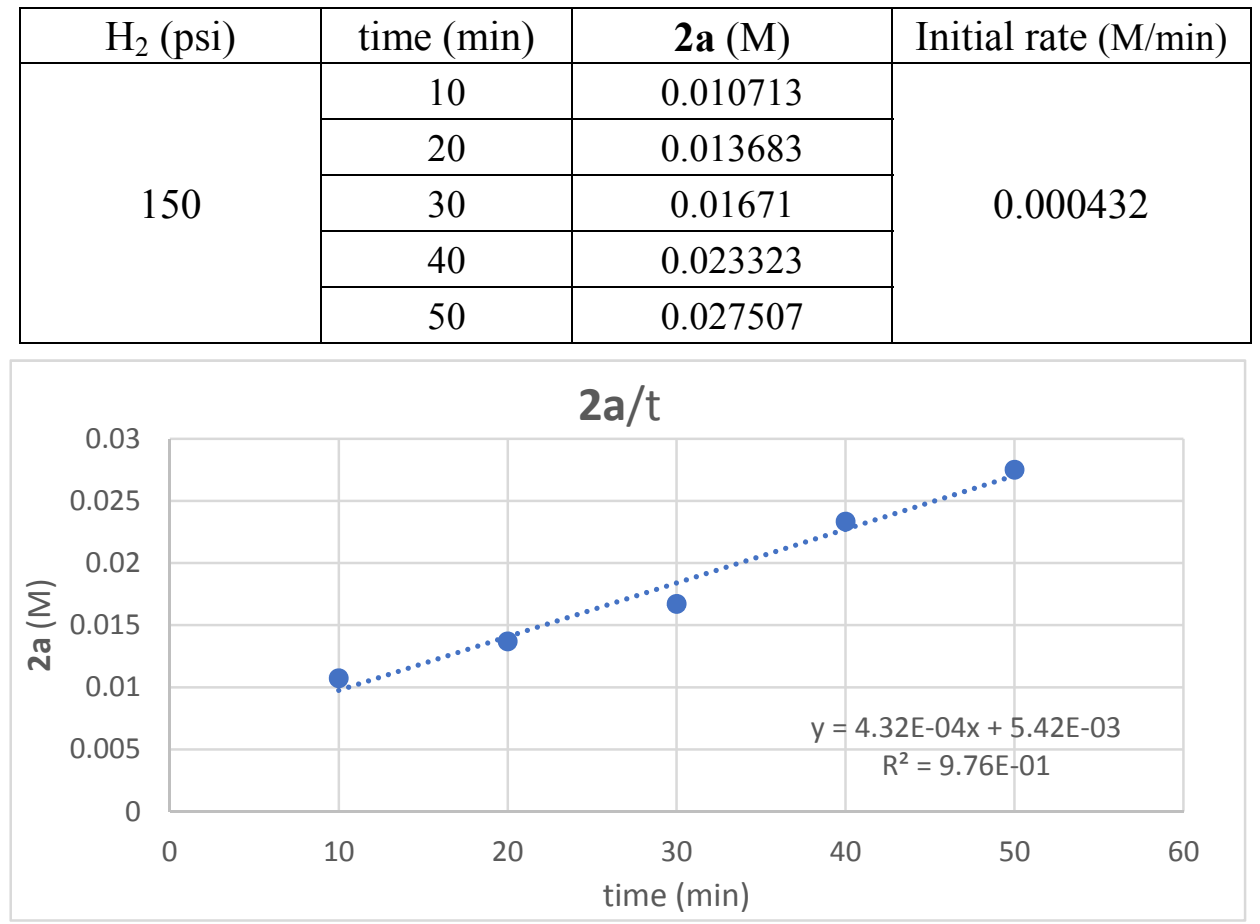

Figure $\mathrm{S}$ 5.2.3.2 Initial rates vs $\mathrm{H}_{2}$ pressure (120 psi)

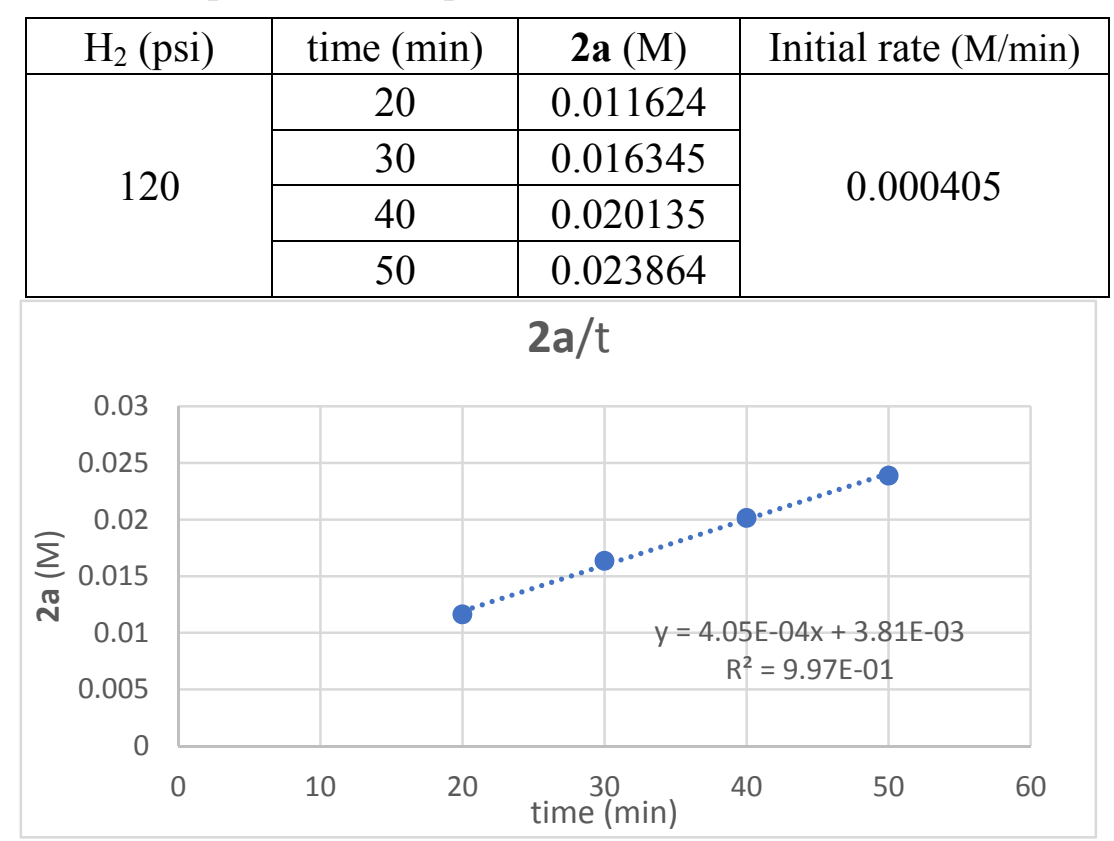

Figure S5.2.3.3 Initial rates vs $\mathrm{H}_{2}$ pressure (90 psi) 


\begin{tabular}{|c|c|c|c|}
\hline $\mathrm{H}_{2}(\mathrm{psi})$ & time $(\mathrm{min})$ & $\mathbf{2 a}(\mathrm{M})$ & Initial rate $(\mathrm{M} / \mathrm{min})$ \\
\hline \multirow{3}{*}{90} & 20 & 0.011594 & \\
\cline { 2 - 3 } & 30 & 0.01624 & \multirow{2}{*}{0.000380} \\
\cline { 2 - 3 } & 40 & 0.020345 & \\
\cline { 2 - 3 } & 50 & 0.022902 & \\
\hline
\end{tabular}

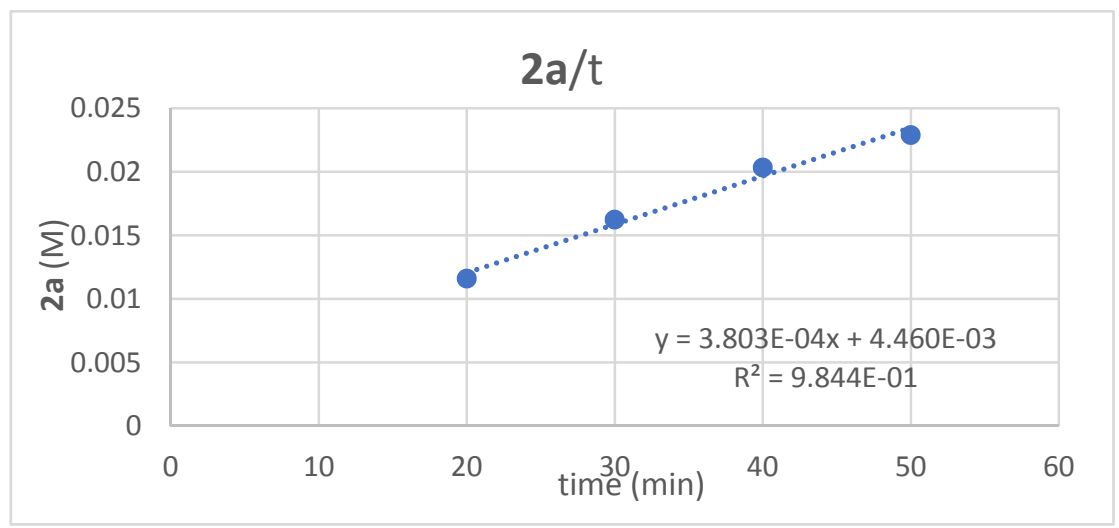

Figure S5.2.3.4 Initial rates vs $\mathrm{H}_{2}$ pressure (60 psi)

\begin{tabular}{|c|c|c|c|}
\hline $\mathrm{H}_{2}(\mathrm{psi})$ & time $(\mathrm{min})$ & $\mathbf{2 a}(\mathrm{M})$ & Initial rate $(\mathrm{M} / \mathrm{min})$ \\
\hline \multirow{3}{*}{60} & 20 & 0.008661 & \multirow{2}{*}{0.000407} \\
\cline { 2 - 3 } & 30 & 0.012917 & \multirow{2}{*}{0.017338} \\
\cline { 2 - 3 } & 40 & 0.020751 & \\
\cline { 2 - 3 } & 50 & 0.04 \\
\hline
\end{tabular}

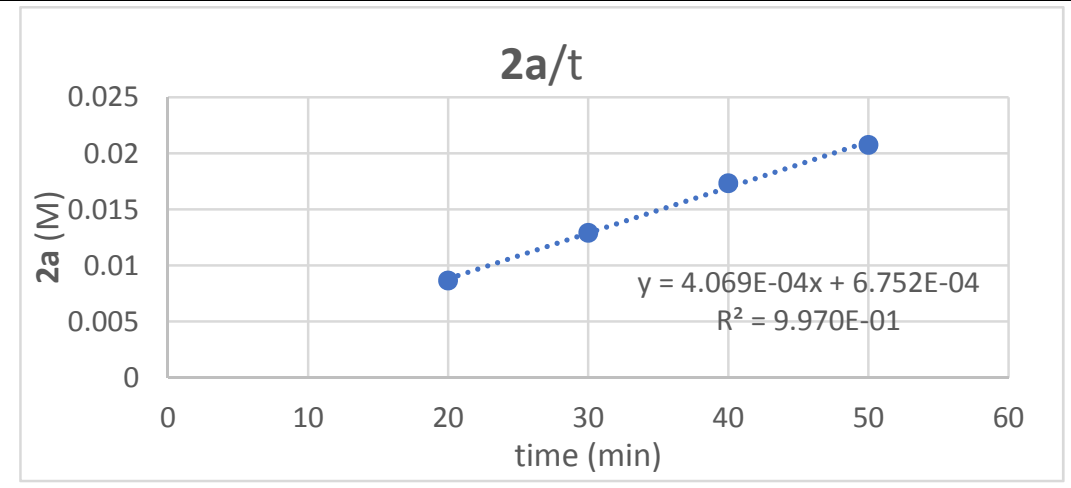

Figure S5.2.3.5 Initial rates vs $\mathrm{H}_{2}$ pressure (45 psi)

\begin{tabular}{|c|c|c|c|}
\hline $\mathrm{H}_{2}(\mathrm{psi})$ & time $(\mathrm{min})$ & $\mathbf{2 a}(\mathrm{M})$ & Initial rate $(\mathrm{M} / \mathrm{min})$ \\
\hline \multirow{4}{*}{45} & 30 & 0.013026 & \multirow{2}{*}{0.000363} \\
\cline { 2 - 3 } & 40 & 0.016307 & \multirow{2}{*}{0.019829} \\
\cline { 2 - 3 } & 50 & 0.023956 & \\
\cline { 2 - 3 } & 60 & 0.036 \\
\hline
\end{tabular}




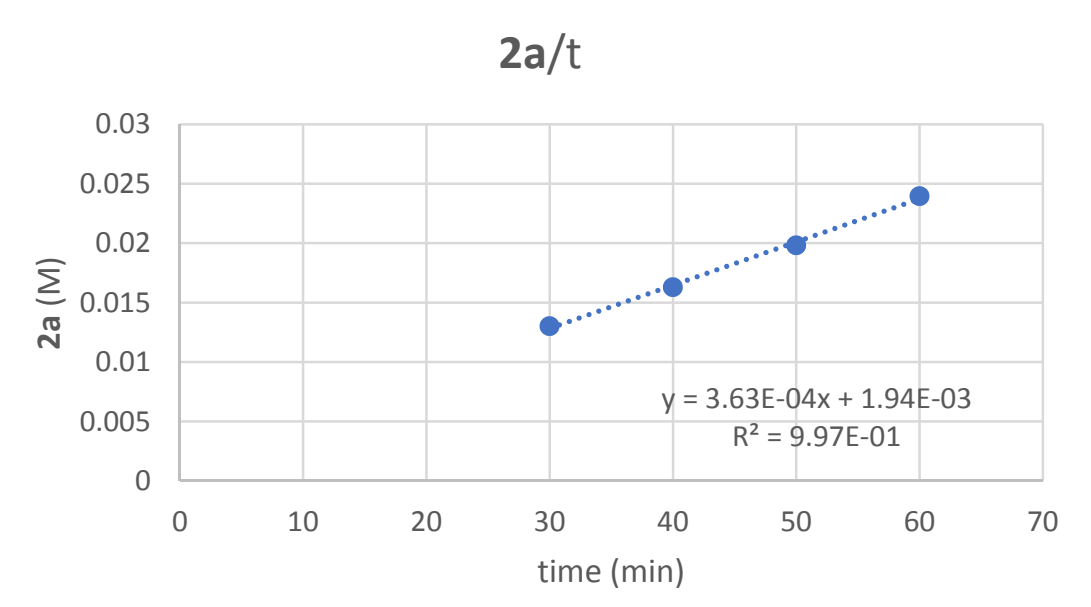

Figure S5.2.3.6 Initial rates vs $\mathrm{H}_{2}$ pressure (30 psi)

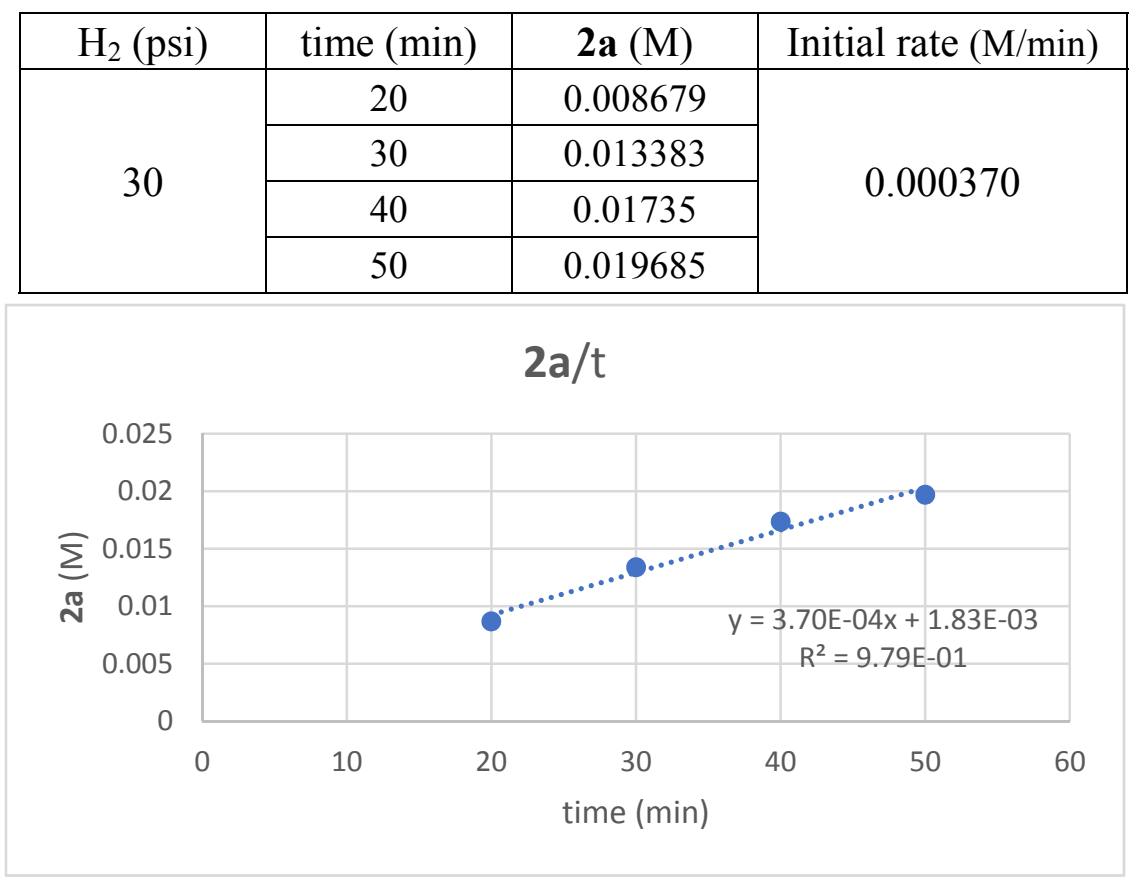

Figure S5.2.3.7 Initial rates vs $\mathrm{H}_{2}$ pressure (25 psi)

\begin{tabular}{|c|c|c|c|}
\hline $\mathrm{H}_{2}(\mathrm{psi})$ & time $(\mathrm{min})$ & $\mathbf{2 a}(\mathrm{M})$ & Initial rate $(\mathrm{M} / \mathrm{min})$ \\
\hline \multirow{4}{*}{25} & 15 & 0.009989 & \\
\cline { 2 - 3 } & 20 & 0.013144 & \\
\cline { 2 - 3 } & 30 & 0.016256 & \multirow{2}{*}{0.000317} \\
\cline { 2 - 3 } & 40 & 0.019405 & \\
\cline { 2 - 3 } & 60 & 0.024779 & \\
\hline
\end{tabular}




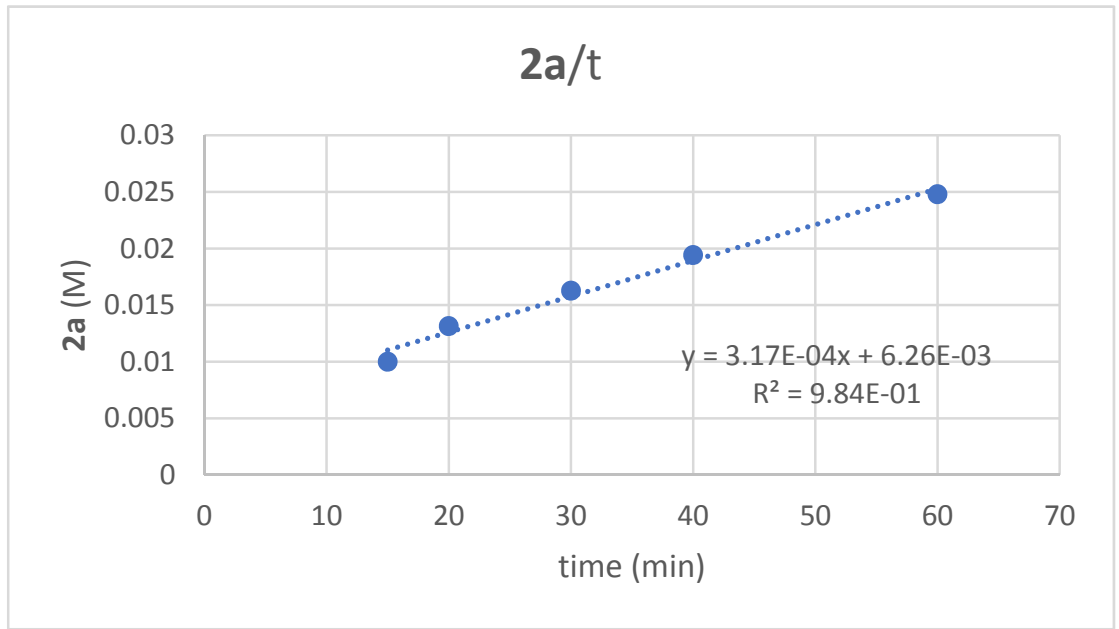

Figure S5.2.3.8 Initial rates vs $\mathrm{H}_{2}$ pressure (20 psi)

\begin{tabular}{|c|c|c|c|}
\hline $\mathrm{H}_{2}(\mathrm{psi})$ & time $(\mathrm{min})$ & $\mathbf{2 a}(\mathrm{M})$ & Initial rate $(\mathrm{M} / \mathrm{min})$ \\
\hline \multirow{4}{*}{20} & 30 & 0.015312 & \\
\cline { 2 - 3 } & 40 & 0.018517 & \multirow{2}{*}{0.000262} \\
\cline { 2 - 3 } & 50 & 0.021578 & \multirow{2}{*}{0.0263} \\
\cline { 2 - 3 } & 60 & 0.02263 & \\
\cline { 2 - 3 } & 70 & 0.026348 & \\
\hline
\end{tabular}

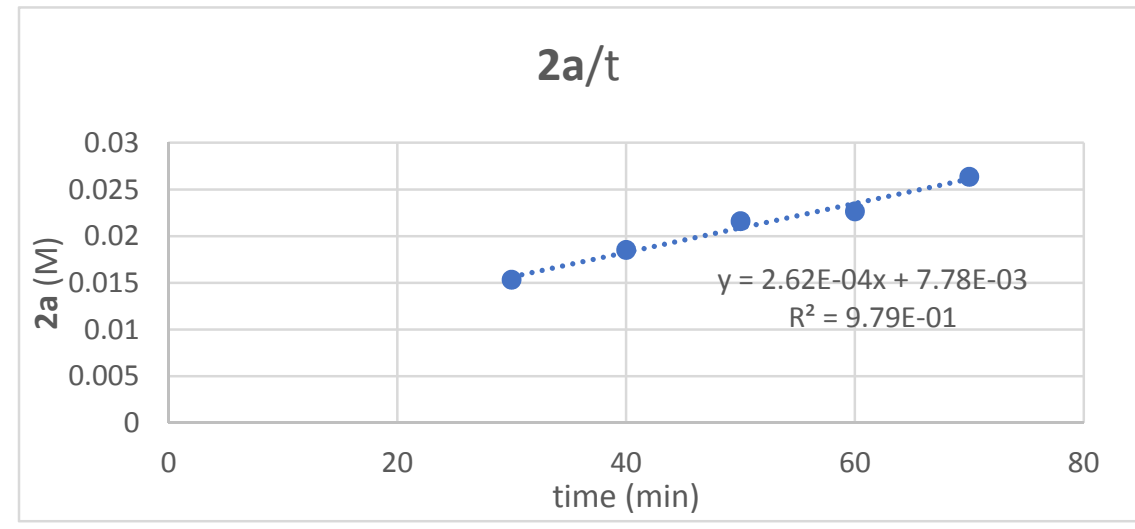

Table S5.2.3 Order for hydrogen gas pressure

\begin{tabular}{ccc}
\hline Entry & $\mathrm{H}_{2}(\mathrm{psi})$ & Rate $(\mathrm{M} / \mathrm{min})$ \\
\hline 1 & 150 & 0.0004320 \\
2 & 120 & 0.0004050 \\
3 & 90 & 0.0003800 \\
4 & 60 & 0.0004069 \\
5 & 45 & 0.0003630 \\
6 & 30 & 0.0003700 \\
7 & 25 & 0.0003170 \\
8 & 20 & 0.0002620 \\
\hline
\end{tabular}




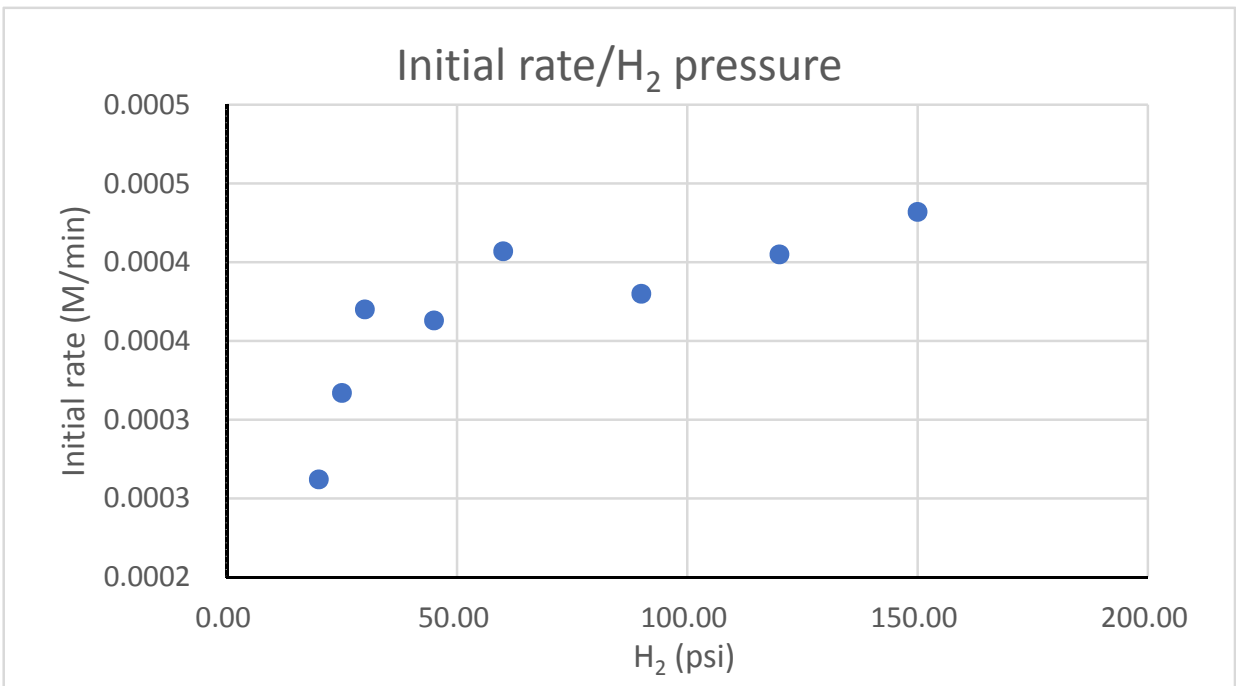

\subsubsection{Initial rates vs [substrate 1a]}

To a Q-tube charged with a stir bar, standard substrate 1a $(33.6 \mathrm{mg}, 0.1 \mathrm{mmol} ; 50.4 \mathrm{mg}, 0.15 \mathrm{mmol} ; 67.3$ $\mathrm{mg}, 0.2 \mathrm{mmol} ; 84.1 \mathrm{mg}, 0.25 \mathrm{mmol})$ and $\mathrm{Ru}(\mathrm{COD}) \mathrm{Cl}_{2}(2.8 \mathrm{mg})$ were added, The Q-tube was then transferred into the glovebox. Distilled 1,4-dioxane $(2.66 \mathrm{~mL})$ was added. The Q-tube was reassembled and transferred out of the glovebox and flushed with hydrogen gas for 10 times then charged with 150 psi hydrogen gas followed by heating at $130{ }^{\circ} \mathrm{C}$ in a pre-heated oil bath. The reaction was stopped at indicated time points by releasing the hydrogen pressure and chilling in an acetone-dry ice bath. The reaction mixture was pass through a Celite pad and flush with DCM, the combined filtration was concentrated in vacuum to afford the crude mixture. To the crude mixture, internal standard (1,1,2,2-tetrachloroethane) was added and the yield of the 1a was determined by ${ }^{1} \mathrm{H}-\mathrm{NMR}$ or GC-FID using tetradecane as internal standard. Zero order dependence on [1a] was observed. Figure S5.2.4.1. Initial rates vs $[\mathbf{1 a}](0.1 \mathrm{mmol})$

\begin{tabular}{|c|c|c|c|}
\hline 1a (M) & time (min) & 2a $(\mathrm{M})$ & Initial rate (M/min) \\
\hline \multirow{4}{*}{0.03759} & 10 & 0.00579 & \\
\cline { 2 - 3 } & 15 & 0.007753 & \multirow{3}{*}{0.000431} \\
\cline { 2 - 3 } & 20 & 0.010355 & \\
\cline { 2 - 3 } & 25 & 0.012859 & \\
\cline { 2 - 3 } & 30 & 0.014017 & \\
\hline
\end{tabular}




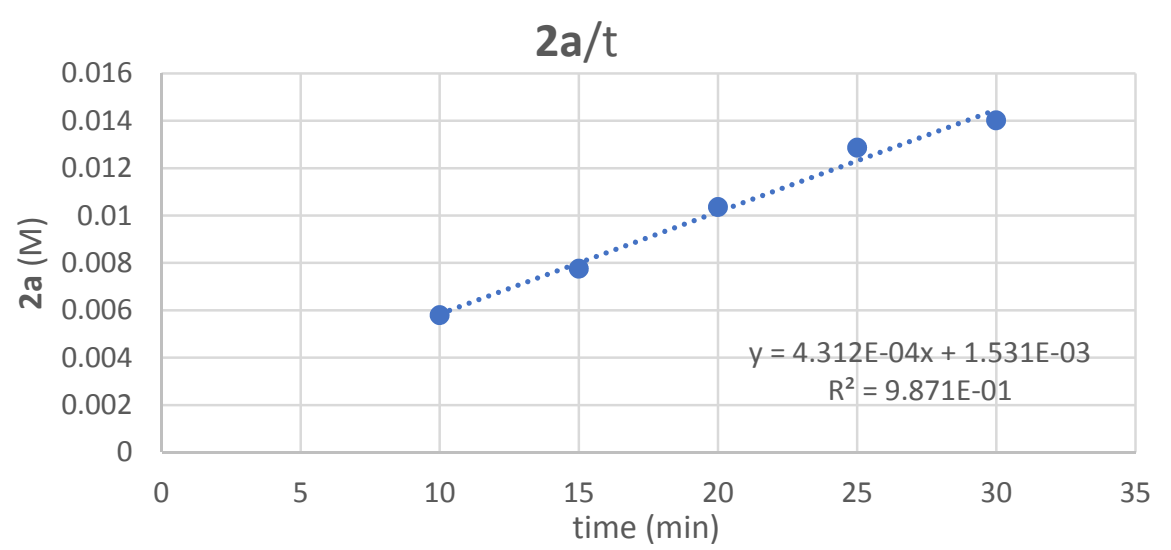

Figure S5.2.4.2. Initial rates vs [1a] $(0.15 \mathrm{mmol})$

\begin{tabular}{|c|c|c|c|}
\hline 1a $(\mathrm{M})$ & time $(\mathrm{min})$ & $\mathbf{2 a}(\mathrm{M})$ & Initial rate $(\mathrm{M} / \mathrm{min})$ \\
\hline \multirow{4}{*}{0.05639} & 10 & 0.007839 & \\
\cline { 2 - 3 } & 15 & 0.010297 & \multirow{3}{*}{0.0005057} \\
\cline { 2 - 3 } & 20 & 0.014132 & \\
\cline { 2 - 3 } & 25 & 0.016636 & \\
\cline { 2 - 3 } & 30 & 0.017312 & \\
\hline
\end{tabular}

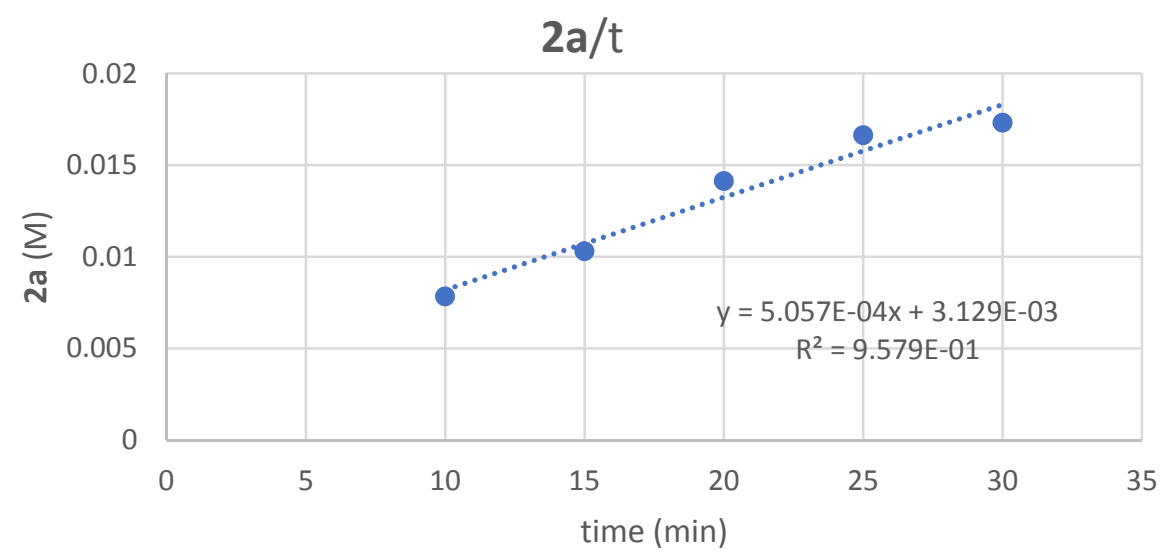

Figure S5.2.4.3. Initial rates vs [1a] $(0.20 \mathrm{mmol})$

\begin{tabular}{|c|c|c|c|}
\hline 1a $(\mathrm{M})$ & time $(\mathrm{min})$ & $\mathbf{2 a}(\mathrm{M})$ & Initial rate $(\mathrm{M} / \mathrm{min})$ \\
\hline \multirow{4}{*}{0.07519} & 10 & 0.010713 & \\
\cline { 2 - 3 } & 20 & 0.013683 & \multirow{3}{*}{0.000432} \\
\cline { 2 - 3 } & 30 & 0.01671 & \\
\cline { 2 - 3 } & 40 & 0.023323 & \\
\cline { 2 - 3 } & 50 & 0.027507 & \\
\hline
\end{tabular}




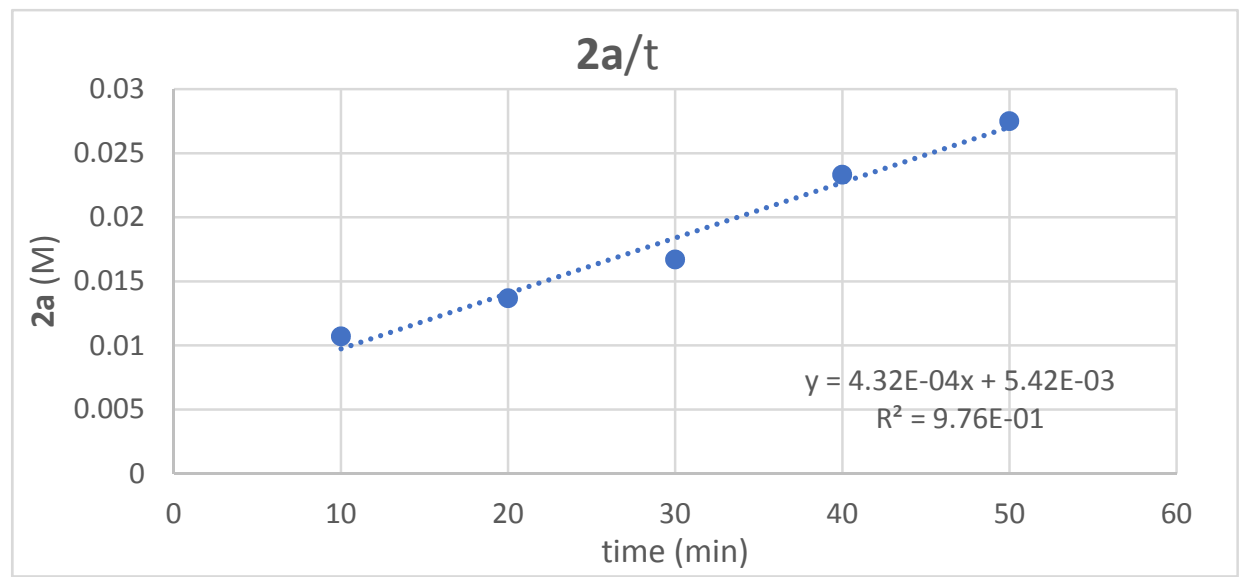

Figure S5.2.4.4. Initial rates vs [1a] $(0.25 \mathrm{mmol})$

\begin{tabular}{|c|c|c|c|}
\hline 1a $(\mathrm{M})$ & time $(\mathrm{min})$ & $\mathbf{2 a}(\mathrm{M})$ & Initial rate $(\mathrm{M} / \mathrm{min})$ \\
\hline \multirow{3}{*}{0.09397} & 10 & 0.008295 & \\
\cline { 2 - 3 } & 20 & 0.014386 & \multirow{2}{*}{0.0004906} \\
\cline { 2 - 3 } & 30 & 0.019449 & \\
\cline { 2 - 3 } & 40 & 0.022961 & \\
\hline
\end{tabular}

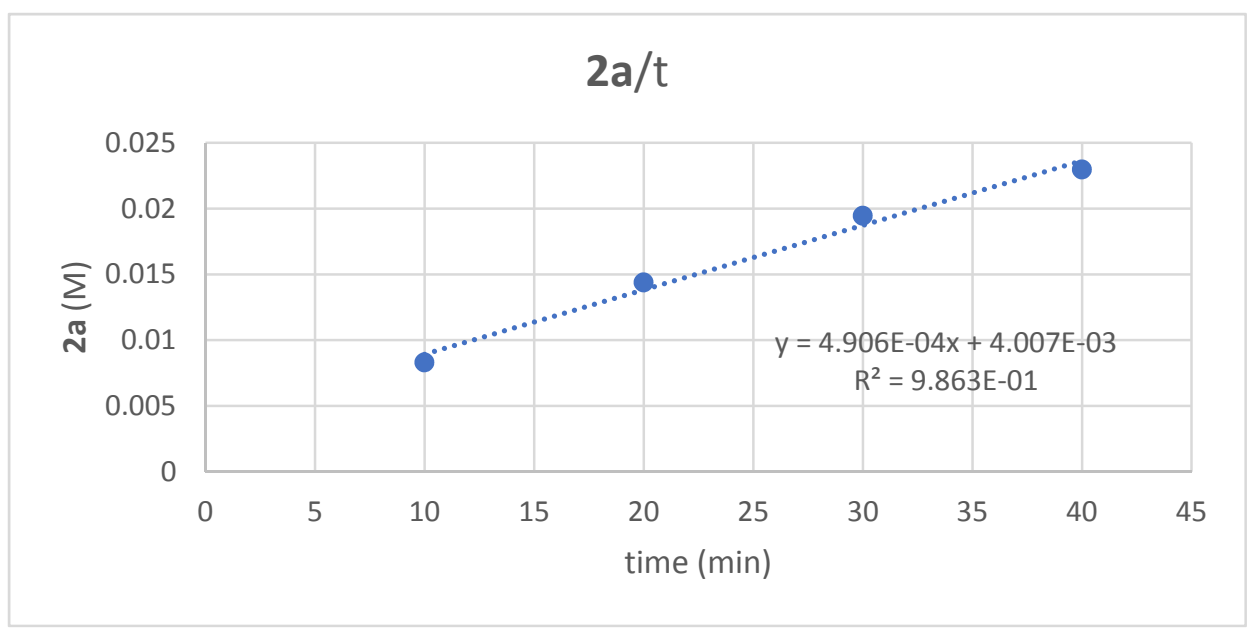

Figure S5.2.4 Order for [1a]

\begin{tabular}{cc}
\hline 1a $(\mathrm{M})$ & rate $(\mathrm{M} / \mathrm{min})$ \\
\hline 0.03759 & 0.000431 \\
0.05639 & 0.000506 \\
0.07519 & 0.000432 \\
0.09397 & 0.000491 \\
\hline
\end{tabular}




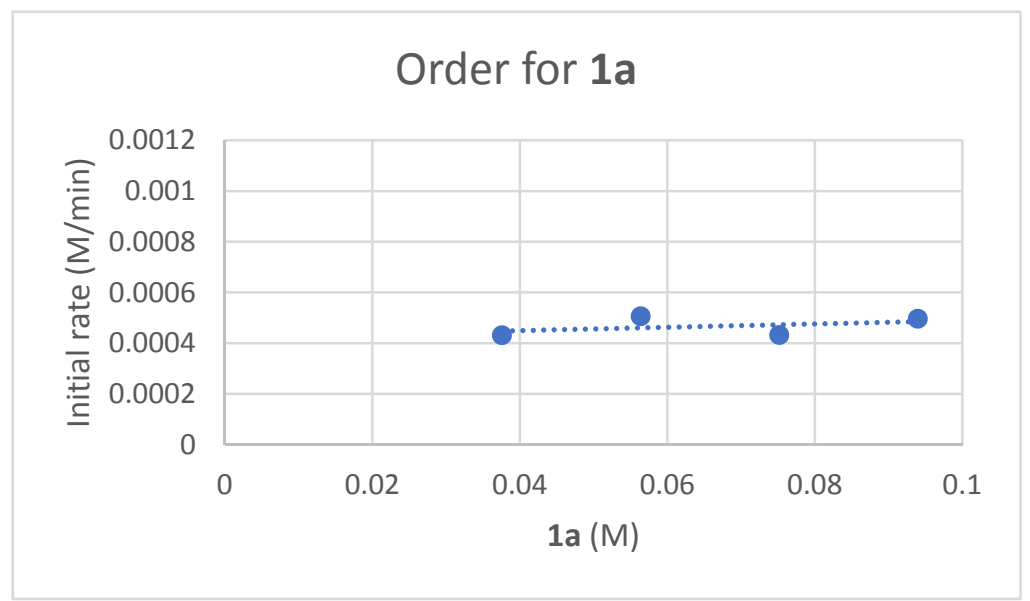

\subsection{Hammett plot analysis}

The Hammett plot analysis was performed by comparing the relative initial rates of product formation of $p$-X or $m$-X substituted substrates to that of the $p-\mathrm{H}$ substituted substrate. The relative initial rates were analyzed by ${ }^{1} \mathrm{H}-\mathrm{NMR}$ using 1,1,2,2-tetrachloroethane as internal standard. A plot of $\log \left(\mathrm{k}_{x} / \mathrm{k}_{H}\right)$ vs. the corresponding $\sigma$ values $^{24}$ resulted in the Hammett plot. Detailed experimental procedures and results are provided as below.

To a Q-tube charged with a stir bar, the indicated substrate $(0.2 \mathrm{mmol})$ and $\mathrm{Ru}(\mathrm{COD}) \mathrm{Cl}_{2}(2.8 \mathrm{mg}, 5 \mathrm{~mol} \%)$ were added, The Q-tube was then transferred into the glovebox. Distilled 1,4-dioxane (2.66 mL) was added. The Q-tube was reassembled, transferred out of the glovebox and flushed with hydrogen gas for 10 times then charged with 150 psi hydrogen gas followed by heating at $130{ }^{\circ} \mathrm{C}$ in a pre-heated oil bath. The reaction was stopped at indicated time points by releasing the hydrogen pressure and chilling in an acetone-dry ice bath. The reaction mixture was pass through a Celite pad and flush with DCM, the combined filtration was concentrated under reduced pressure to afford the crude mixture. To the crude mixture, internal standard (1,1,2,2-tetrachloroethane, $\sim 31 \mathrm{mg}$ ) was added and the yield of the product was determined by ${ }^{1} \mathrm{H}-\mathrm{NMR}$ or GC-FID using tetradecane as internal standard.

5.3.1 Initial rates of substrate 1ah $(p-\mathrm{OMe})$

\begin{tabular}{|c|c|c|c|}
\hline & time (min) & 2ah (M) & Initial rate $(\mathrm{M} / \mathrm{min})$ \\
\hline \multirow{5}{*}{$\begin{array}{l}\mathrm{OMe} \\
1 \text { ah }\end{array}$} & 30 & 0.010722 & \multirow{5}{*}{0.0003024} \\
\hline & 40 & 0.013233 & \\
\hline & 50 & 0.015744 & \\
\hline & 60 & 0.019805 & \\
\hline & 70 & 0.022556 & \\
\hline
\end{tabular}




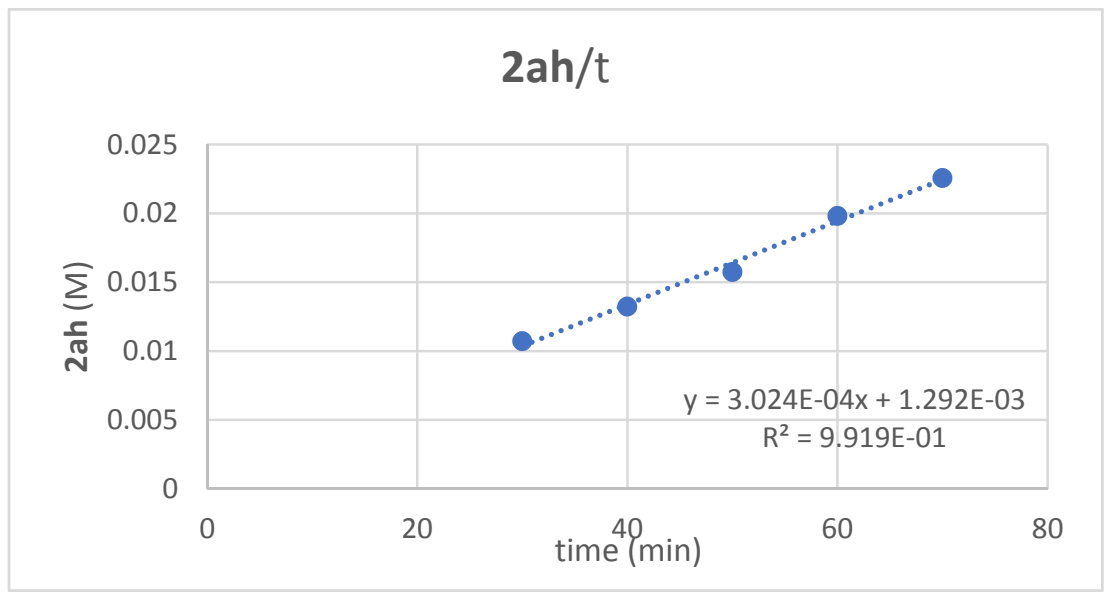

5.3.2 Initial rates of substrate $\mathbf{1 f}(p-\mathrm{Me})$

\begin{tabular}{|c|c|c|c|}
\hline & time (min) & $2 f(M)$ & Initial rate $(\mathrm{M} / \mathrm{min})$ \\
\hline \multirow{5}{*}{$\begin{array}{l}\text { Me } \\
\text { if }\end{array}$} & 20 & 0.008722 & \multirow{5}{*}{0.0003953} \\
\hline & 30 & 0.01385 & \\
\hline & 40 & 0.018647 & \\
\hline & 50 & 0.020331 & \\
\hline & 60 & 0.025248 & \\
\hline
\end{tabular}

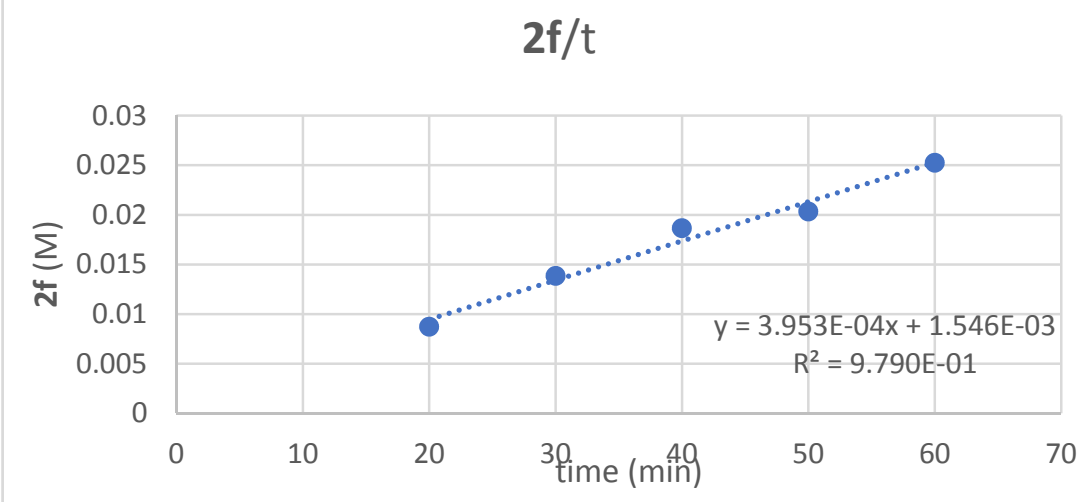

5.3.3 Initial rates of substrate $1 \mathbf{a}(p-\mathrm{H})$

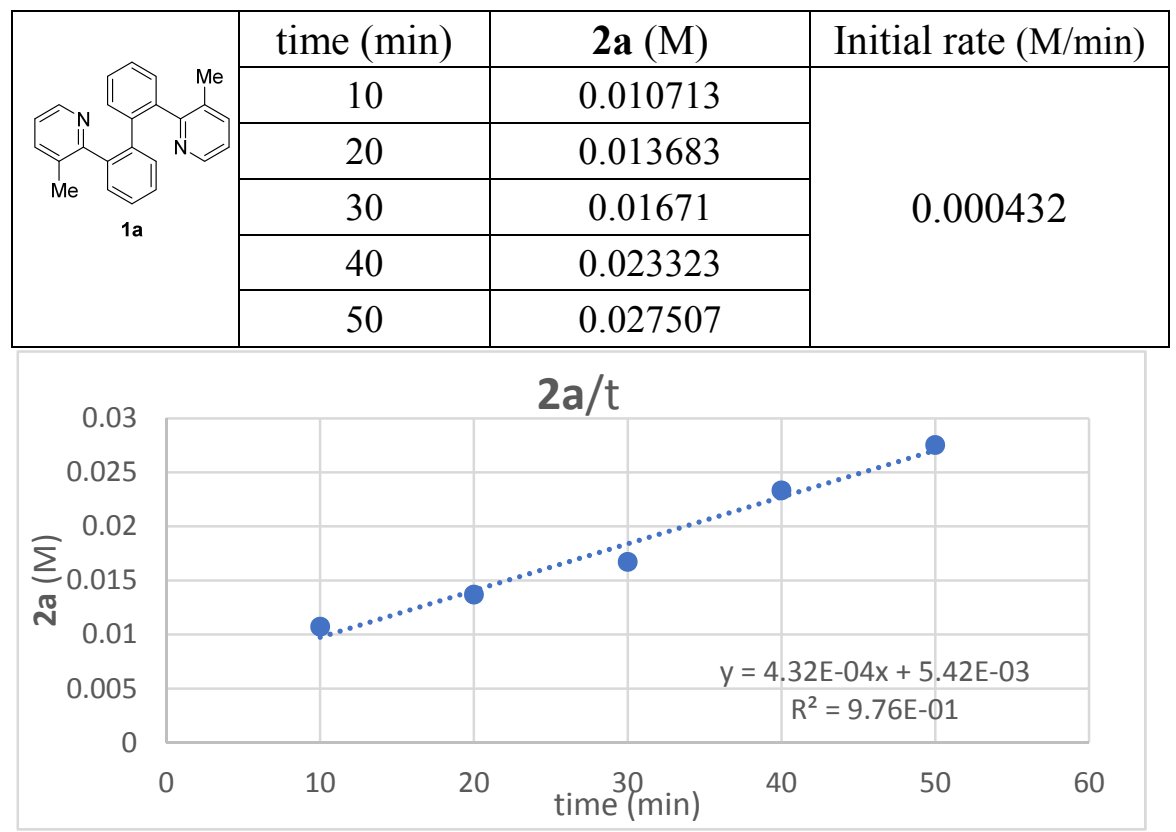


5.3.4 Initial rates of substrate 1ai $(p-\mathrm{Cl})$

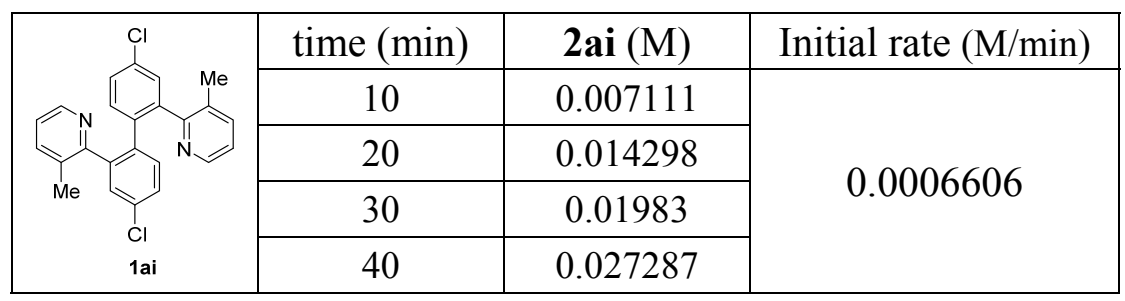

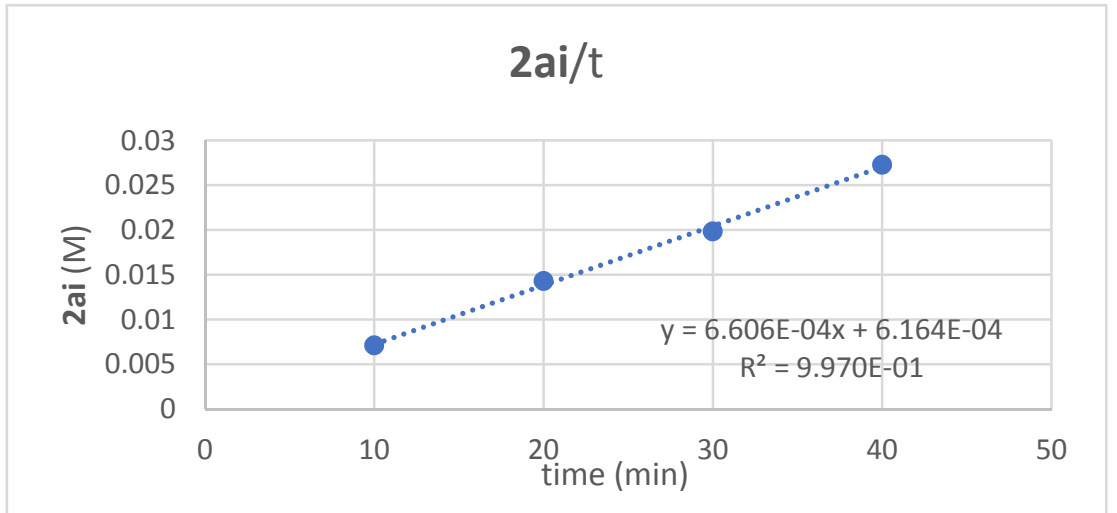

5.3.5 Initial rates of substrate $2 \mathbf{l}\left(m-\mathrm{CF}_{3}\right)$

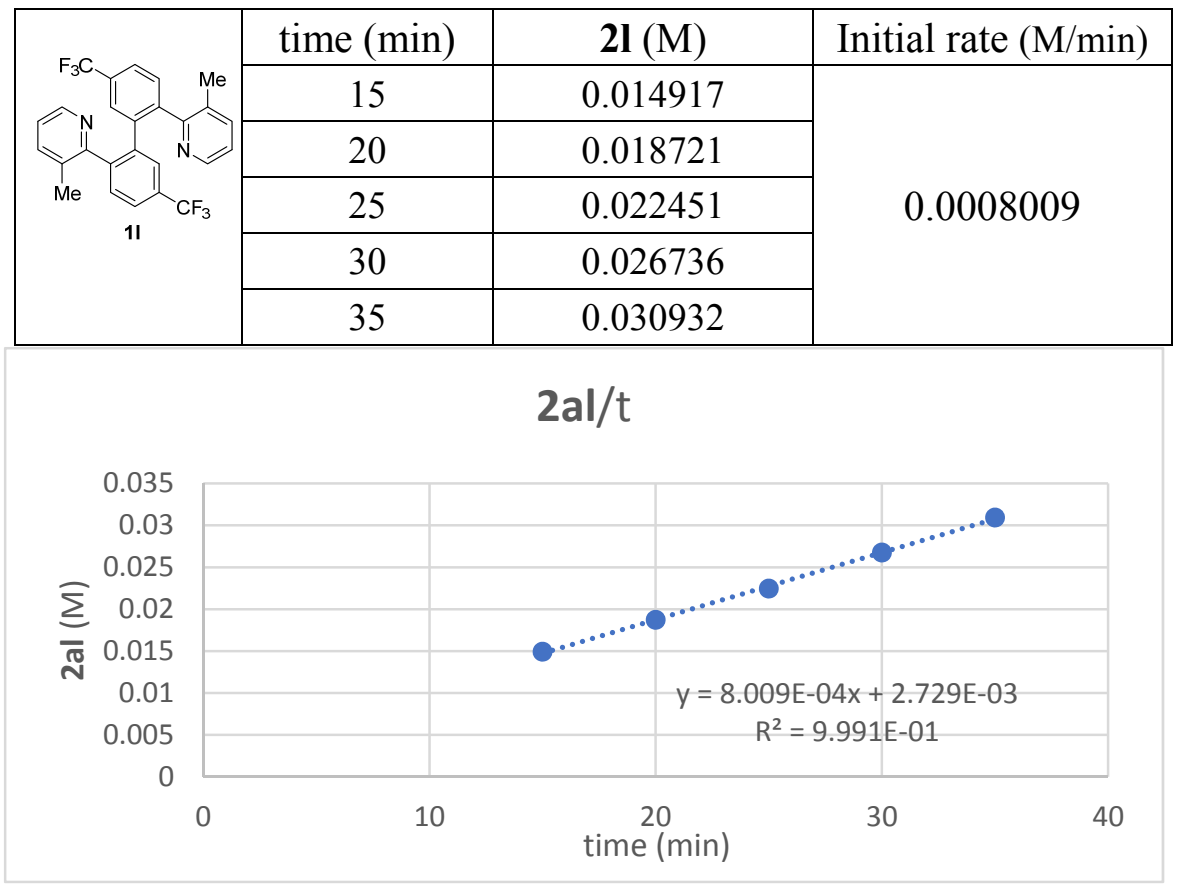

\section{Figure S5.3 Hammett plot}

\begin{tabular}{|c|c|c|c|c|}
\hline & $\sigma$ & $\log \left(\mathrm{K}_{\mathrm{x}} / \mathrm{K}_{\mathrm{H}}\right)$ & $\mathrm{K}$ & $\mathrm{K}_{\mathrm{x}} / \mathrm{K}_{\mathrm{H}}$ \\
\hline$p-\mathrm{OMe}$ & -0.27 & -0.15490196 & 0.0003024 & 0.7 \\
\hline$p-\mathrm{Me}$ & -0.17 & -0.03855693 & 0.0003953 & 0.915046296 \\
\hline $\mathrm{H}$ & 0 & 0 & 0.000432 & 1 \\
\hline$p-\mathrm{Cl}$ & 0.23 & 0.184454823 & 0.0006606 & 1.529166667 \\
\hline$m-\mathrm{CF}_{3}$ & 0.43 & 0.268094547 & 0.0008009 & 1.853935185 \\
\hline
\end{tabular}




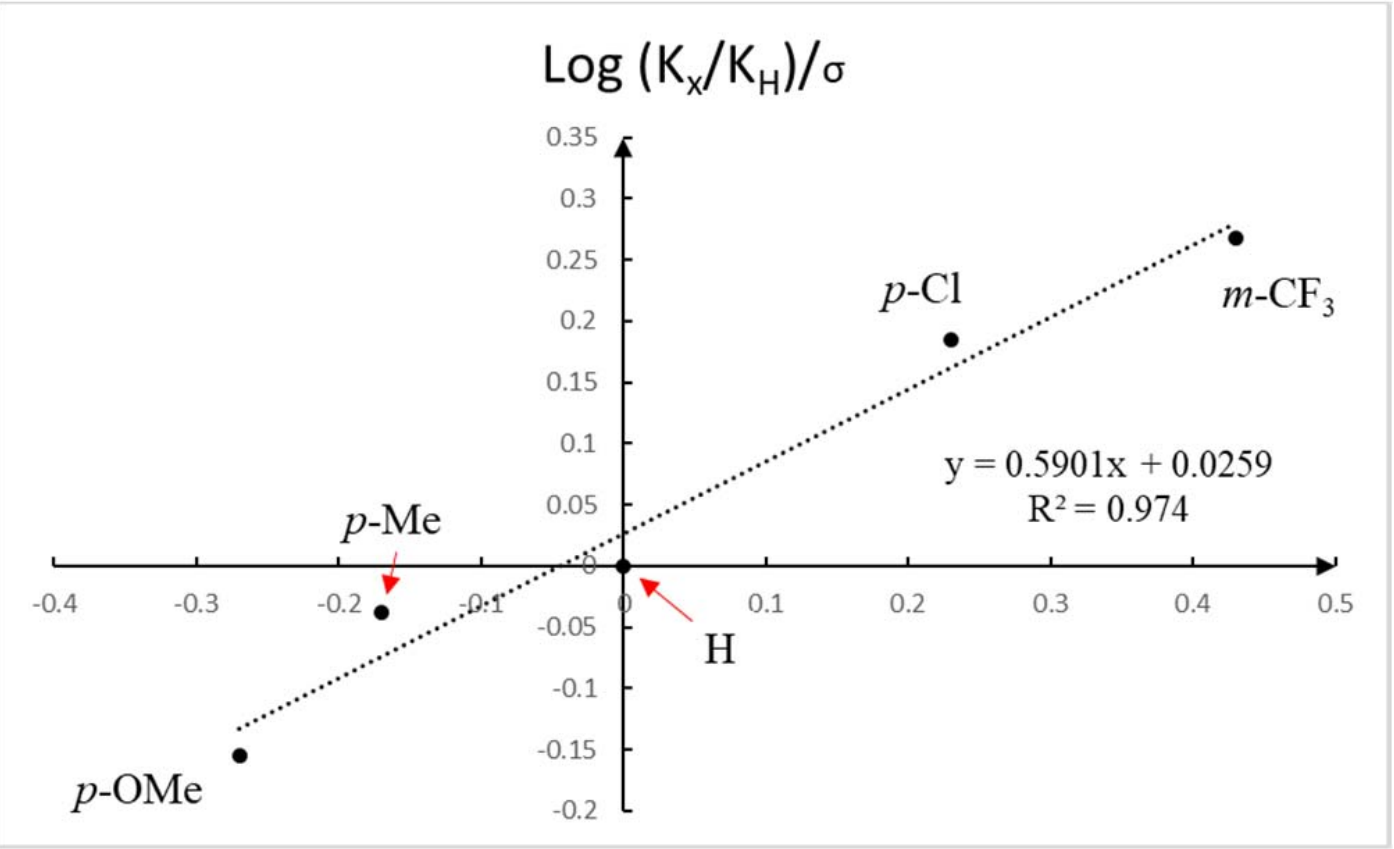

\subsection{Resting state}

\subsubsection{Capturing the resting state}

To a Q-tube charged with a stir bar, substrate 1 ah $(79.3 \mathrm{mg}, 0.2 \mathrm{mmol})$ and $\mathrm{Ru}(\mathrm{COD}) \mathrm{Cl}_{2}(16.8 \mathrm{mg}, 30 \mathrm{~mol} \%)$ were added, The Q-tube was then transferred into the glovebox. Distilled 1,4-dioxane (2.66 mL) was added. The Q-tube was reassembled, transferred out of the glovebox and flushed with hydrogen gas for 10 times then charged with 150 psi hydrogen gas followed by heating at $130{ }^{\circ} \mathrm{C}$ in a pre-heated oil bath for 20 min. The reaction was stopped by releasing the hydrogen pressure and chilling in an acetone-dry ice bath. The Q-tube was then transferred into the glovebox, the reaction mixture was filtered to afford a dark green solid which was washed by dry 1,4-dioxane $(1.0 \mathrm{~mL})$ for 3 times and dried in vacuum to give complex 5ah $(\sim 10 \mathrm{mg}, \sim 29 \%$ yield based on $\left.\mathrm{Ru}(\mathrm{COD}) \mathrm{Cl}_{2}\right)$ as a dark green solid. ${ }^{1} \mathbf{H} \mathbf{N M R}\left(\mathrm{CD}_{2} \mathrm{Cl}_{2}, 400 \mathrm{MHz}\right) \delta 8.09(\mathrm{~d}, J=5.0 \mathrm{~Hz}, 2 \mathrm{H})$, $7.63(\mathrm{~d}, J=7.9 \mathrm{~Hz}, 1 \mathrm{H}), 7.11(\mathrm{dd}, J=7.9,5.2 \mathrm{~Hz}, 2 \mathrm{H}), 7.06(\mathrm{~d}, J=2.6 \mathrm{~Hz}, 2 \mathrm{H}), 7.00(\mathrm{dd}, J=9.3,2.6 \mathrm{~Hz}, 1 \mathrm{H})$, $6.56(\mathrm{~d}, J=9.3 \mathrm{~Hz}, 2 \mathrm{H}), 3.85(\mathrm{~s}, 6 \mathrm{H}), 2.32(\mathrm{~s}, 6 \mathrm{H}) .{ }^{13} \mathbf{C} \mathbf{N M R}\left(\mathrm{CD}_{2} \mathrm{Cl}_{2}, 100 \mathrm{MHz}\right) \delta 161.3,156.7,148.5,138.9$, $138.3,133.0,125.7,124.2,112.8,97.7,88.3,56.3,16.9$. The solid was collected and crystalized in 1,4-dioxane and hexane to afford the crystal that was used by X-Ray crystallography. 


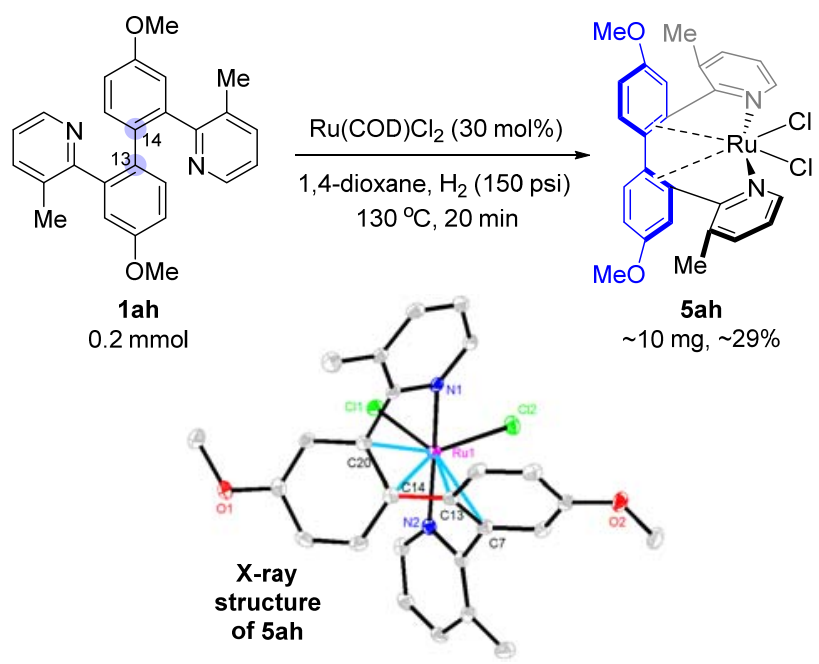

\subsubsection{Monitoring the resting state}

To a Q-tube charged with a stir bar, substrate $\mathbf{1 a h}(79.3 \mathrm{mg}, 0.2 \mathrm{mmol})$ and $\mathrm{Ru}(\mathrm{COD}) \mathrm{Cl}_{2}(16.8 \mathrm{mg}, 30$ mol\%) were added, The Q-tube was then transferred into the glovebox. Distilled 1,4-dioxane (2.66 mL) was added. The Q-tube was reassembled, transferred out of the glovebox and flushed with hydrogen gas for 10 times then charged with 150 psi hydrogen gas followed by heating at $130{ }^{\circ} \mathrm{C}$ in a pre-heated oil bath. The reaction was stopped at indicated time points by releasing the hydrogen pressure and chilling in an acetone-dry ice bath. The Q-tube was then transferred into the glovebox, and the reaction mixture was concentrated in vacuum. To the crude mixture, internal standard (1,1,2,2-tetrachloroethane, $\sim 31 \mathrm{mg})$ was added and the yield of $\mathbf{2 a h}$ was determined by ${ }^{1} \mathrm{H}-\mathrm{NMR}$. The ${ }^{1} \mathrm{H}-\mathrm{NMR}$ spectrum of the crude mixture was compared with that of the isolated 5ah. Note that 5ah was observable from the very beginning to almost the end of the reaction.
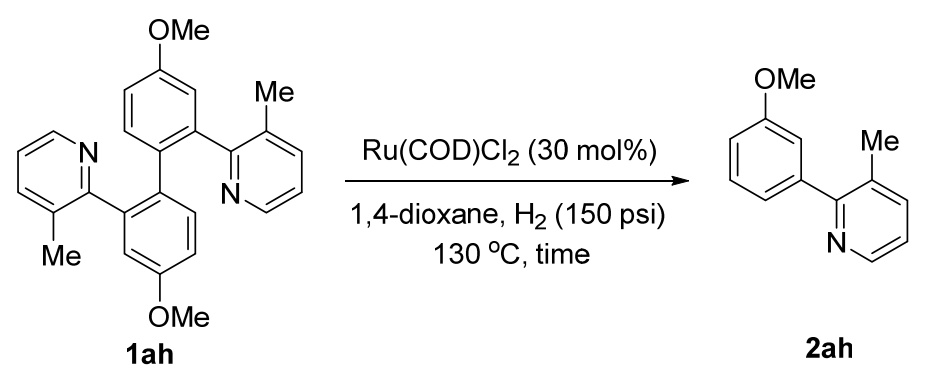

\begin{tabular}{|cc|}
\hline time & 2ah yield \\
$20 \mathrm{~min}$ & $33 \%$ \\
$40 \mathrm{~min}$ & $49 \%$ \\
$60 \mathrm{~min}$ & $68 \%$ \\
$100 \mathrm{~min}$ & $87 \%$ \\
$140 \mathrm{~min}$ & $90 \%$ \\
\hline
\end{tabular}

2ah 


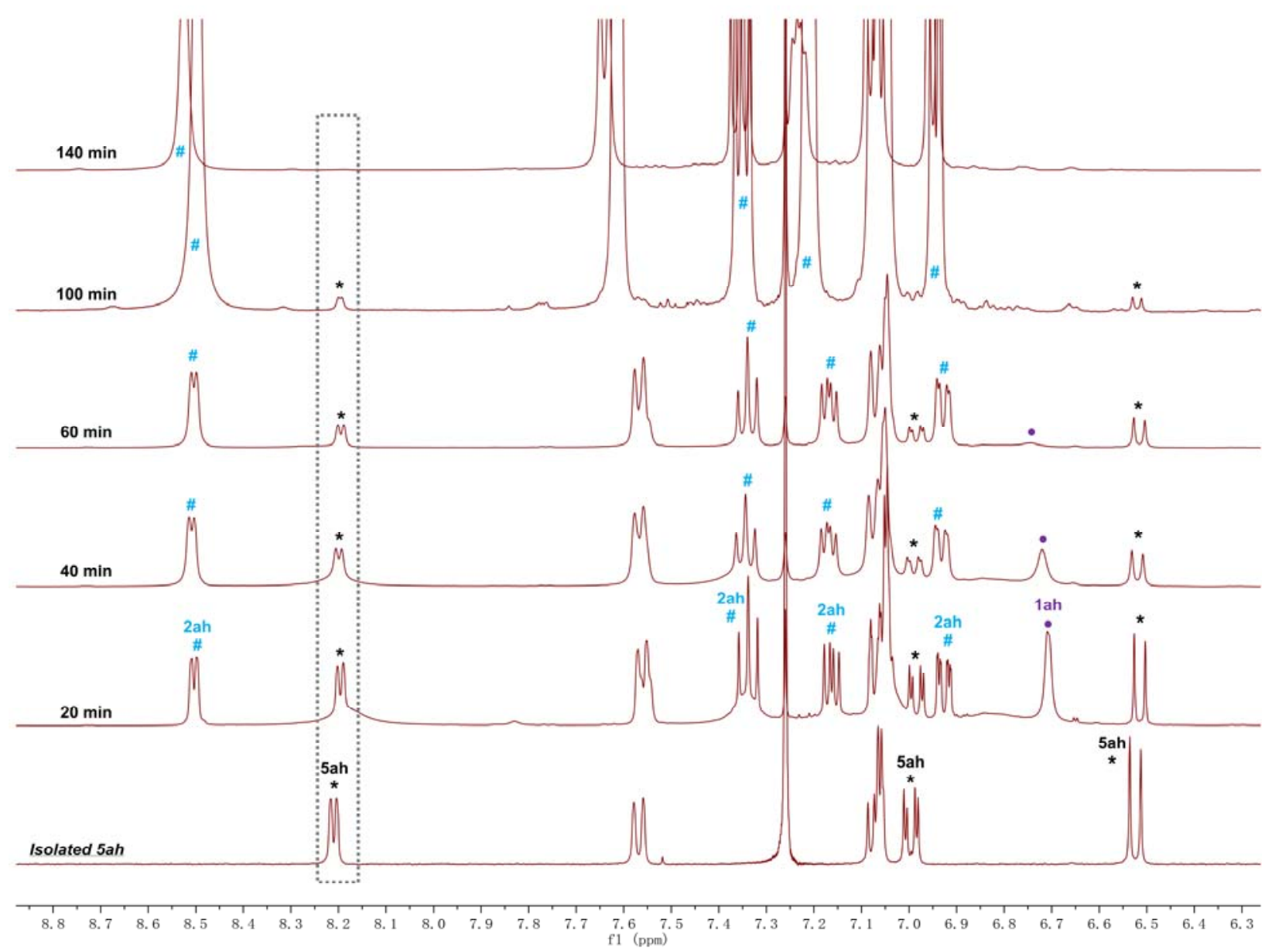

\subsubsection{Other control experiments}

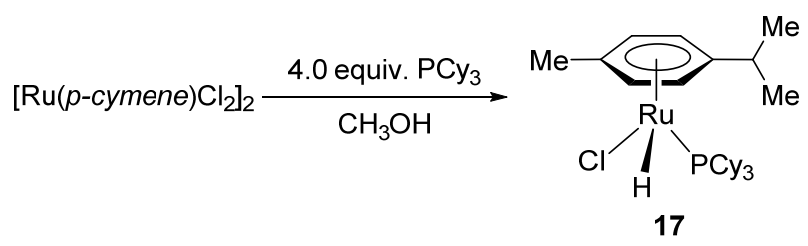

Ruthenium monohydride complex 17 was synthesized by following the literature reported method ${ }^{25}$. To a solution of $\left[\mathrm{Ru}(p \text {-cymene }) \mathrm{Cl}_{2}\right]_{2}(400 \mathrm{mg}, 0.65 \mathrm{mmol})$ in $\mathrm{MeOH}(35 \mathrm{~mL}), \mathrm{PCy}_{3}(732 \mathrm{mg}, 2.61 \mathrm{mmol})$ was added in the glovebox. The reaction mixture was stirred for $12 \mathrm{~h}$ at room temperature. The product precipitated in the form of an orange powder, which was isolated by filtration, washed with methanol and pentane, and dried under vacuum (yield: $380 \mathrm{mg}, 53 \%) .{ }^{1} \mathbf{H}$ NMR $\left(500 \mathrm{MHz}, \mathrm{CD}_{2} \mathrm{Cl}_{2}\right) \delta 6.09(\mathrm{t}, J=5.7 \mathrm{~Hz}, 2 \mathrm{H}), 4.51(\mathrm{~d}, J$ $=6.0 \mathrm{~Hz}, 1 \mathrm{H}), 4.31(\mathrm{~d}, J=5.6 \mathrm{~Hz}, 1 \mathrm{H}), 2.26-2.17(\mathrm{~m}, 1 \mathrm{H}), 2.04-1.95(\mathrm{~m}, 6 \mathrm{H}), 1.94-1.75(\mathrm{~m}, 12 \mathrm{H}), 1.74-$ $1.66(\mathrm{~m}, 3 \mathrm{H}), 1.58-1.46(\mathrm{~m}, 6 \mathrm{H}), 1.34(\mathrm{~d}, J=6.8 \mathrm{~Hz}, 3 \mathrm{H}), 1.30-1.11(\mathrm{~m}, 12 \mathrm{H}),-8.00(\mathrm{~d}, J=49.7 \mathrm{~Hz}, 1 \mathrm{H})$. ${ }^{13}$ C NMR (126 MHz, $\left.\mathrm{CD}_{2} \mathrm{Cl}_{2}\right) \delta 106.5,97.9,91.2(\mathrm{~d}, J=2.4 \mathrm{~Hz}), 86.9(\mathrm{~d}, J=4.8 \mathrm{~Hz}), 86.7(\mathrm{~d}, J=4.3 \mathrm{~Hz})$, $79.6(\mathrm{~d}, J=2.9 \mathrm{~Hz}), 36.8(\mathrm{~d}, J=22.1 \mathrm{~Hz}), 31.8,30.1,30.0,28.4(\mathrm{~d}, J=10.6 \mathrm{~Hz}), 28.3(\mathrm{~d}, J=10.0 \mathrm{~Hz}), 27.3$, 25.4, 21.3, 19.4. ${ }^{31} \mathbf{P}$ NMR $\left(202 \mathrm{MHz}, \mathrm{CD}_{2} \mathrm{Cl}_{2}\right) \delta 59.1$. The analytic data match the literature ${ }^{26}$. 


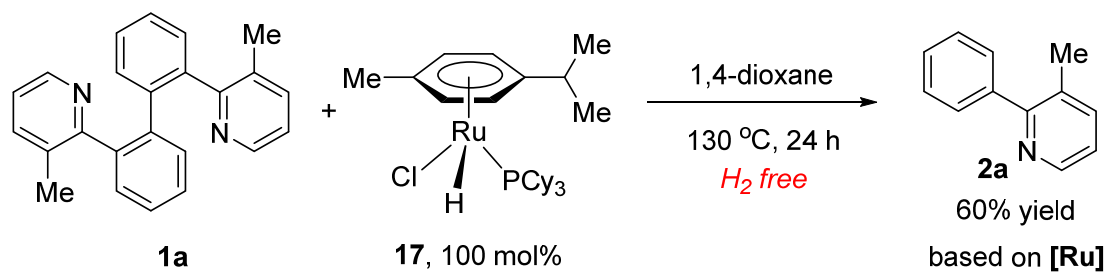

To a vial charged with a stir bar, substrate 1a $(67.3 \mathrm{mg}, 0.2 \mathrm{mmol})$, monohydride complex 17 (110.4 $\mathrm{mg}$, $0.2 \mathrm{mmol}$ ) and $2.0 \mathrm{~mL}$ of 1,4-dioxane were added in glovebox. The vial was sealed and stirred on a pre-heated heating block at $130{ }^{\circ} \mathrm{C}$ for $24 \mathrm{~h}$. Upon completion of the reaction, the reaction mixture was transferred in to a flask, concentrated under reduced pressure and directly purified using column chromatography on silica gel to afford $20.3 \mathrm{mg}$ of $\mathbf{2 a}$ (60\% yield) based on ruthenium monohydride 17.

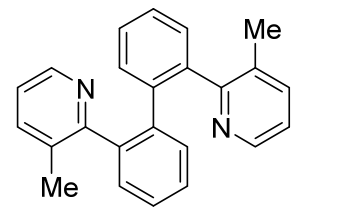

1a

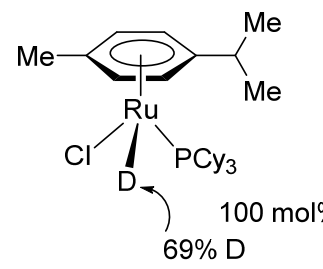

17-D

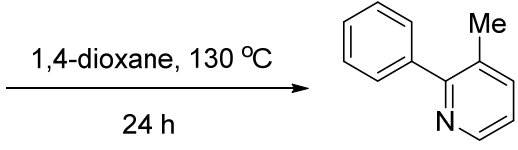

2a, $65 \%$

no $D$ incorporated

17-D was synthesized by stirring $17(300 \mathrm{mg})$ in $\mathrm{DCM} / \mathrm{CD}_{3} \mathrm{OD}(1 / 4, \mathrm{v} / \mathrm{v}) 15 \mathrm{~mL}$ for 3 days. The solution was then concentrated in vacuum to give 17-D with $69 \% \mathrm{D}$ incorporated (determined by ${ }^{1} \mathrm{H}$ NMR). To a vial charged with a stir bar, substrate 1a $(33.6 \mathrm{mg}, 0.1 \mathrm{mmol}), \mathbf{1 7}-\mathrm{D}(55.3 \mathrm{mg}, 0.1 \mathrm{mmol})$ and $1.0 \mathrm{~mL}$ of 1,4-dioxane were added in glovebox. The vial was sealed and stirred on a pre-heated heating block at $130{ }^{\circ} \mathrm{C}$ for $24 \mathrm{~h}$. Upon completion of the reaction, the reaction mixture was transferred in to a flask, concentrated under reduced pressure and directly purified using column chromatography on silica gel to afford $11.0 \mathrm{mg}$ of 2a (65\% yield) based on 17-D. ${ }^{1} \mathrm{H}$ NMR indicated that there is no D incorporation in the product.

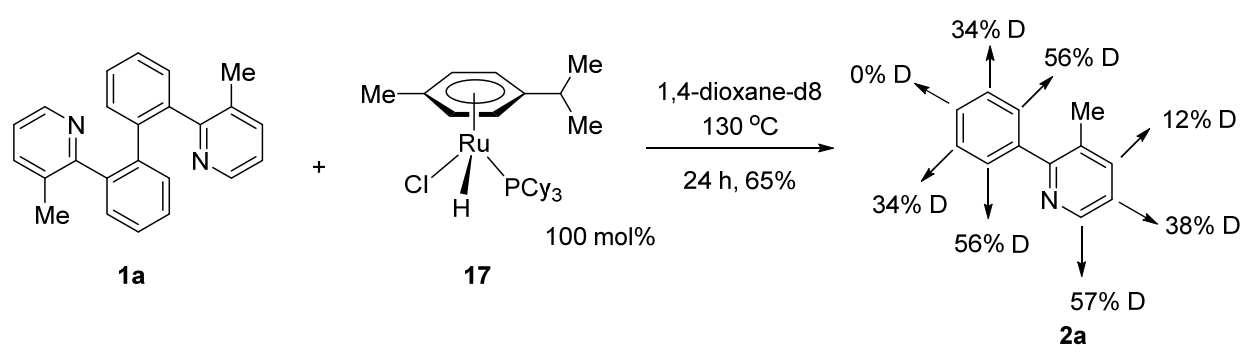

To a vial charged with a stir bar, substrate $1 \mathrm{a}(33.6 \mathrm{mg}, 0.1 \mathrm{mmol}), 17(55.2 \mathrm{mg}, 0.1 \mathrm{mmol})$ and $1.0 \mathrm{~mL}$ of 1,4-dioxane- $\mathrm{d}_{8}$ were added in glovebox. The vial was sealed and stirred on a pre-heated heating block at 130 ${ }^{\circ} \mathrm{C}$ for $24 \mathrm{~h}$. Upon completion of the reaction, the reaction mixture was transferred in to a flask, concentrated under reduced pressure and directly purified using column chromatography on silica gel to afford $11.0 \mathrm{mg}$ of 2a (65\% yield) based on $\mathbf{1 7}$. The $\mathrm{D}$ incorporation in the product was measured by ${ }^{1} \mathrm{H}$ NMR. 


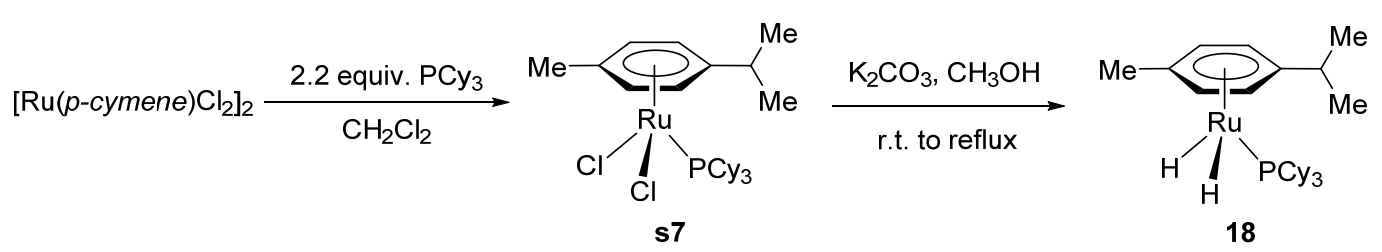

Ruthenium complex $\mathbf{s} 7$ was synthesized by following the literature reported method ${ }^{27}$. To a solution of $\left[\mathrm{Ru}(\text { p-cymene }) \mathrm{Cl}_{2}\right]_{2}(1.0 \mathrm{~g}, 1.63 \mathrm{mmol})$ in $\mathrm{CH}_{2} \mathrm{Cl}_{2}(10 \mathrm{~mL}), \mathrm{PCy}_{3}(1.0 \mathrm{mg}, 3.56 \mathrm{mmol})$ was added in the glovebox. The reaction mixture was stirred for $6 \mathrm{~h}$ at room temperature. The volume of the dark red solution was reduced to one third and slow addition of pentane $(20 \mathrm{~mL})$ provided red color precipitate. which was isolated by filtration, washed with pentane, and dried under vacuum (yield: $1.4 \mathrm{~g}, 73 \%)$. ${ }^{1} \mathbf{H}$ NMR (500 MHz, $\left.\mathrm{CDCl}_{3}\right) \delta 5.62-5.49(\mathrm{~m}, 4 \mathrm{H}), 2.89-2.77(\mathrm{~m}, 1 \mathrm{H}), 2.41(\mathrm{q}, J=12.1 \mathrm{~Hz}, 3 \mathrm{H}), 2.17-2.04(\mathrm{~m}, 6 \mathrm{H}), 2.08(\mathrm{~s}, 3 \mathrm{H})$, $1.88-1.66(\mathrm{~m}, 9 \mathrm{H}), 1.53-1.37(\mathrm{~m}, 6 \mathrm{H}), 1.36-1.16(\mathrm{~m}, 12 \mathrm{H}), 1.29$ (d, $J=6.5 \mathrm{~Hz}, 3 \mathrm{H}) .{ }^{31} \mathbf{P}$ NMR $(202 \mathrm{MHz}$, $\left.\mathrm{CDCl}_{3}\right) \delta 25.3$. The analytic data match the literature ${ }^{27 .}$

A mixture of $\mathbf{s} 7(1.4 \mathrm{~g}, 2.38 \mathrm{mmol}), \mathrm{K}_{2} \mathrm{CO}_{3}(0.7 \mathrm{~g}, 5.07 \mathrm{mmol})$ and methanol $(20 \mathrm{~mL})$ was stirred at room temperature for $0.5 \mathrm{~h}$ and the resulting yellow slurry was heated at reflux for $2 \mathrm{~h}$. The hot solution was immediately filtered. Upon cooling down, pale yellow solid precipitated from the brown filtrate which was isolated by filtration, washed with methanol and pentane to afford the titled dihydride complex 18 (240 $\mathrm{mg}$, 20\%). ${ }^{1} \mathbf{H}$ NMR (500 MHz, $\left.\mathrm{C}_{6} \mathrm{D}_{6}\right) \delta 5.17(\mathrm{~d}, J=5.6 \mathrm{~Hz}, 2 \mathrm{H}), 5.09$ (d, $\left.J=5.6 \mathrm{~Hz}, 2 \mathrm{H}\right), 2.51-2.42(\mathrm{~m}, 1 \mathrm{H})$, 2.09 (s, 3H), $2.08-2.01(\mathrm{~m}, 6 \mathrm{H}), 1.88-1.73(\mathrm{~m}, 6 \mathrm{H}), 1.70-1.58(\mathrm{~m}, 6 \mathrm{H}), 1.47-1.36(\mathrm{~m}, 6 \mathrm{H}), 1.30(\mathrm{~d}, J=$ $6.9 \mathrm{~Hz}, 6 \mathrm{H}), 1.26-1.14(\mathrm{~m}, 9 \mathrm{H}),-10.98(\mathrm{~d}, J=43.3 \mathrm{~Hz}, 2 \mathrm{H}) .{ }^{13} \mathbf{C}$ NMR $\left(101 \mathrm{MHz}, \mathrm{C}_{6} \mathrm{D}_{6}\right) \delta 110.0(\mathrm{~d}, J=2.6$ $\mathrm{Hz}), 96.5,85.3(\mathrm{~d}, J=2.3 \mathrm{~Hz}), 79.7(\mathrm{~d}, J=3.9 \mathrm{~Hz}), 39.7(\mathrm{~d}, J=21.8 \mathrm{~Hz}), 33.1,30.8,28.3(\mathrm{~d}, J=10.0 \mathrm{~Hz})$, 27.4, 24.9, 21.3. ${ }^{31} \mathbf{P}$ NMR $\left.\left(202 \mathrm{MHz}, \mathrm{C}_{6} \mathrm{D}_{6}\right)\right) \delta$ 77.5. The analytic data match the literature ${ }^{26}$.

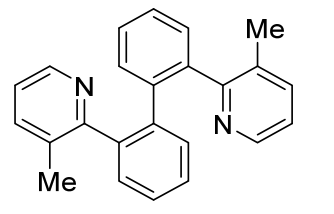

$1 \mathbf{a}$

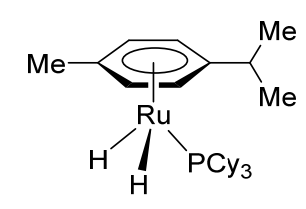

18

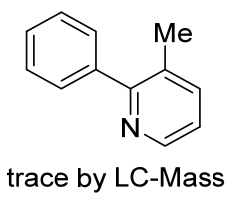

2a

To a vial charged with a stir bar, substrate $1 \mathrm{a}$ (33.6 mg, $0.1 \mathrm{mmol})$, ruthenium dihydride complex 18 (51.7 $\mathrm{mg}, 0.1 \mathrm{mmol}$ ) and $1.0 \mathrm{~mL}$ of 1,4-dioxane were added in glovebox. The vial was sealed and stirred on a pre-heated heating block at $130{ }^{\circ} \mathrm{C}$ for $24 \mathrm{~h}$. Upon completion of the reaction, the reaction mixture was passed through a short pad of silica gel and flashed with EtOAc. The crude mixture was subjected to the LC-MS analysis directly, only a trace amount of monomer $\mathbf{2 a}$ was detected.

\subsubsection{Examining the Ru(0) pathway}




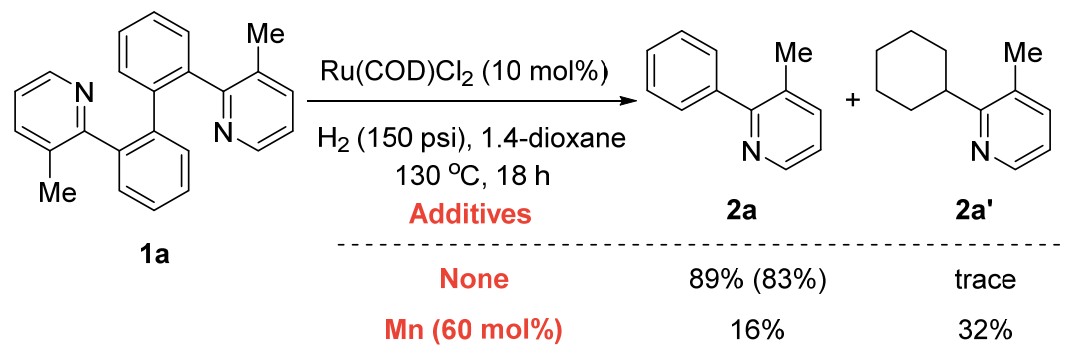

To a Q-tube charged with a stir bar, $\mathbf{1 a}(67.3 \mathrm{mg}, 0.2 \mathrm{mmol}), \mathrm{Ru}(\mathrm{COD}) \mathrm{Cl}_{2}(5.6 \mathrm{mg}, 0.02 \mathrm{mmol})$ and $\mathrm{Mn}$ (6.6 $\mathrm{mg}, 0.12 \mathrm{mmol}$ ) were added. The Q-tube was then transferred into the glovebox. Distilled 1,4-dioxane $(2.66 \mathrm{~mL})$ was added. The Q-tube was reassembled and transferred out of the glovebox. The reaction mixture was in situ freeze-pump-thawed using standard technique for three times. The Q-tube was then charged with $150 \mathrm{psi}$ hydrogen gas followed by heating at $130{ }^{\circ} \mathrm{C}$ in a pre-heated oil bath for $18 \mathrm{~h}$. Upon completion of the reaction, the reaction mixture was transferred in to a flask, concentrated under reduced pressure to give the crude mixture that was roughly purified using column chromatography on silica gel to remove manganese (Eluent: hexane/ethyl acetate $=4 / 1$ ). The combined eluent was concentrated to give the crude mixture that was analyzed by ${ }^{1} \mathrm{H}$ NMR using 1,1,2,2-tetrachloroethane as the internal standard to determine the yield of 2a and 2a'. 2a': colorless oil. ${ }^{1} \mathbf{H}$ NMR $\left(400 \mathrm{MHz}, \mathrm{CDCl}_{3}\right) \delta 8.40(\mathrm{dd}, J=4.6,1.4 \mathrm{~Hz}, 1 \mathrm{H}), 7.42-7.34(\mathrm{~m}, 1 \mathrm{H}), 6.98$ (dd, $J=7.6,4.8 \mathrm{~Hz}, 1 \mathrm{H}), 2.91-2.78(\mathrm{~m}, 1 \mathrm{H}), 2.32(\mathrm{~s}, 3 \mathrm{H}), 1.86(\mathrm{dt}, J=19.8,8.1 \mathrm{~Hz}, 2 \mathrm{H}), 1.80-1.61(\mathrm{~m}, 5 \mathrm{H})$, $1.49-1.29$ (m, 3H). ${ }^{13} \mathbf{C}$ NMR (101 MHz, $\left.\mathrm{CDCl}_{3}\right) \delta 164.1,146.6,137.6,129.9,120.7,41.9,31.7,26.7,26.0$, 18.7. The ${ }^{13} \mathrm{C}$ NMR match the literature ${ }^{28}$. 


\section{X-ray data}

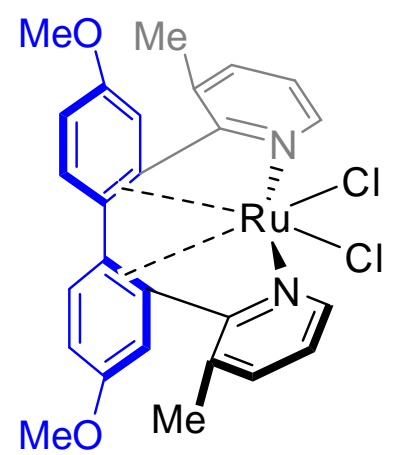

5 ah

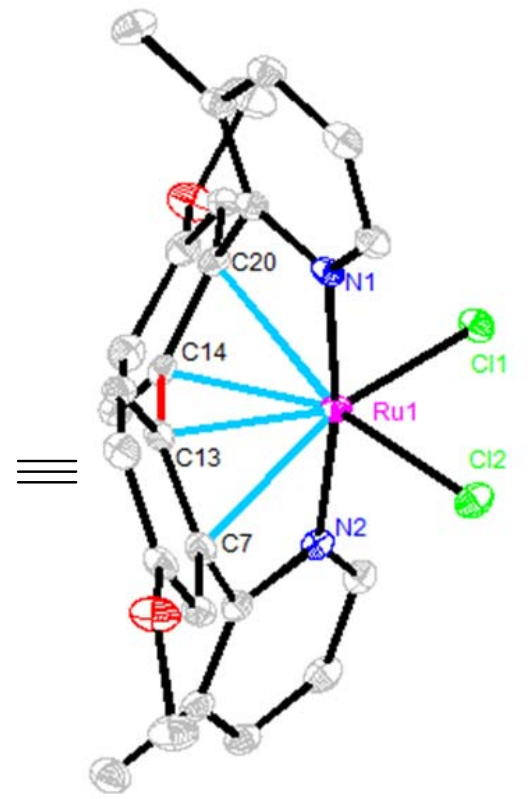

1,4-Dioxane in the crystal structure was omitted for clarity

Figure S6 Crystal data and structure refinement for 5ah.

Identification code

Empirical formula

Formula weight

Temperature/K

Crystal system

Space group

$\mathrm{a} / \AA$

$\mathrm{b} / \AA$

$\mathrm{c} / \AA$

$\alpha /^{\circ}$

$\beta /{ }^{\circ}$

$\gamma /{ }^{\circ}$

Volume $/ \AA^{3}$

Z

$\rho_{\text {calc }} \mathrm{g} / \mathrm{cm}^{3}$

$\mu / \mathrm{mm}^{-1}$

$\mathrm{F}(000)$

Crystal size $/ \mathrm{mm}^{3}$

Radiation
mo_0838_Dong_0m

$\mathrm{C}_{21} \mathrm{H}_{28} \mathrm{ClNO}_{5} \mathrm{Ru}_{0.5}$

460.43

$100(2)$

triclinic

P-1

$10.7706(6)$

$12.8085(7)$

$15.9271(9)$

96.760(2)

$104.610(2)$

94.684(2)

2097.2(2)

4

1.458

0.561

960.0

$0.67 \times 0.21 \times 0.17$

$\operatorname{MoK} \alpha(\lambda=0.71073)$

$2 \Theta$ range for data collection/ ${ }^{\circ} 4.472$ to 56.742

Index ranges

$-14 \leq \mathrm{h} \leq 14,-16 \leq \mathrm{k} \leq 17,-21 \leq 1 \leq 21$ 


\begin{tabular}{|c|c|}
\hline Reflections collected & 59521 \\
\hline Independent reflections & $10276\left[\mathrm{R}_{\mathrm{int}}=0.0435, \mathrm{R}_{\text {sigma }}=0.0419\right]$ \\
\hline Data/restraints/parameters & $10276 / 0 / 522$ \\
\hline Goodness-of-fit on $\mathrm{F}^{2}$ & 1.032 \\
\hline Final $R$ indexes $[\mathrm{I}>=2 \sigma(\mathrm{I})]$ & $\mathrm{R}_{1}=0.0324, \mathrm{wR}_{2}=0.0628$ \\
\hline Final $\mathrm{R}$ indexes [all data] & $\mathrm{R}_{1}=0.0484, \mathrm{wR}_{2}=0.0672$ \\
\hline Largest diff. peak/hole / e & $0.68 /-0.52$ \\
\hline
\end{tabular}




\section{DFT Calculation}

\subsection{Computational Details}

All DFT calculations were performed with Gaussian $09 .^{29}$ Geometry optimizations were performed in the gas phase with the B3LYP functional and a mixed basis set of LANL2DZ for Ru and 6-31G(d) for other atoms. Single point energies were calculated with the M06 functional ${ }^{30}$ and a mixed basis set of SDD for Ru and $6-311+G(d, p)$ for other atoms. Solvation energy corrections were calculated using the SMD model ${ }^{31}$ with 1,4-dioxane as the solvent. The 3D structures were prepared using CYLView. ${ }^{32}$

\subsection{Additional Computational Results}

\subsubsection{Off-cycle $\mathrm{RuCl}_{2}$ species}

Our calculations show that the off-cycle 5a-dimer can dissociate to the active catalyst species 5a with 6.5 $\mathrm{kcal} / \mathrm{mol}$ uphill in free energy (Figure S7.2.1). This leads to the geometry change of the two pyridine DGs from the cis geometry in 5a-dimer to the trans geometry in 5a. The $\mathrm{Ru}-\mathrm{C}$ distances are significantly decreased to 2.42 $\AA ̊$ in 5a from 3.8-4.0 $\AA$ in 5a-dimer. As discussed in the manuscript, the agostic $\mathrm{C}-\mathrm{C} / \mathrm{Ru}$ interaction promotes the subsequent $\mathrm{C}($ aryl $)-\mathrm{C}($ aryl) oxidative addition step. In addition, several complexes (5a-1, 5a-2 and 5a-3) formed from $\mathrm{H}_{2}$ coordination to the monomeric 5a are less favorable than the separated $\mathbf{5 a}$ and $\mathrm{H}_{2}$.

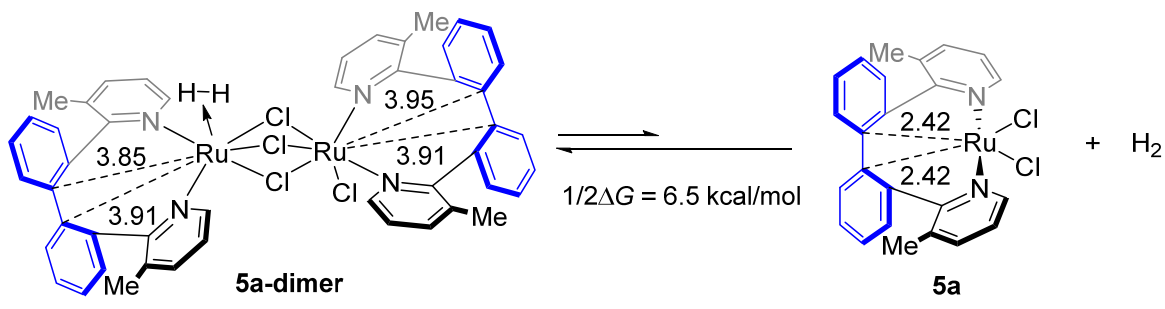

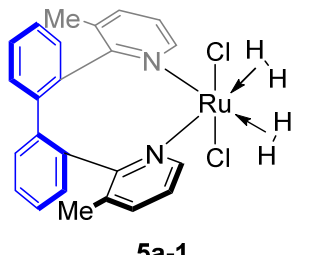

$\Delta G=4.4 \mathrm{kcal} / \mathrm{mol}$

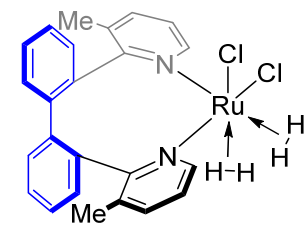

5a-2 $\Delta G=6.6 \mathrm{kcal} / \mathrm{mol}$

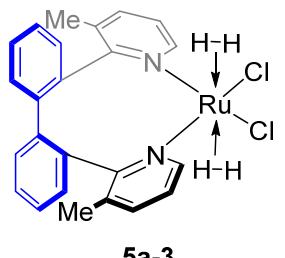

$\Delta G=8.8 \mathrm{kcal} / \mathrm{mol}$

Figure S7.2.1. Possible $\mathrm{RuCl}_{2}$ structures. Energies of 5a-1, 5a-2 and 5a-3 are with respect to the separated 5a and $\mathrm{H}_{2}$.

\subsubsection{Oxidative addition of the $\mathrm{C}(\operatorname{aryl})-\mathrm{C}(\operatorname{aryl})$ in substrates with one pyridine directing group}

To verify the importance of two pyridine groups serving as directing groups in C(aryl)-C(aryl) cleavage, we compared the oxidative addition of the $\mathrm{C}(\operatorname{aryl})-\mathrm{C}(\operatorname{aryl})$ bond in 1a and 1af with the RuHCl species (Figure S7.2.2). The two pyridine DGs trans to each other in $\mathbf{5 b}$ and TS2b can place the target C(aryl)-C(aryl) bond 
closer to the Ru center, leading to a lower barrier (TS2b, $\Delta G^{\ddagger}=12.2 \mathrm{kcal} / \mathrm{mol}$ ). In contrast, only one pyridine DG in TS2b-1 and two cis pyridine DGs in 5b-1 are incapable of drawing the C(aryl)-C(aryl) bond to the Ru center, thus giving a higher barrier (TS2b-1, $\left.\Delta G^{\ddagger}=22.5 \mathrm{kcal} / \mathrm{mol}\right)$. In addition, the electron donation of the two pyridine substituents is more helpful for stabilizing the formed $\mathrm{Ru}(\mathrm{II})$-aryl intermediate than one pyridine substituent.

(a)

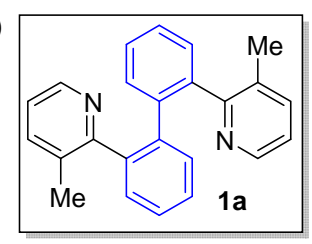

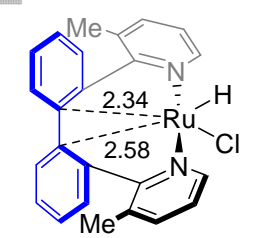

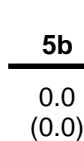

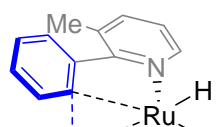

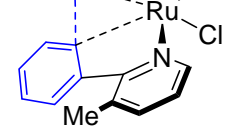

12.2

$(9.5)$ (b)
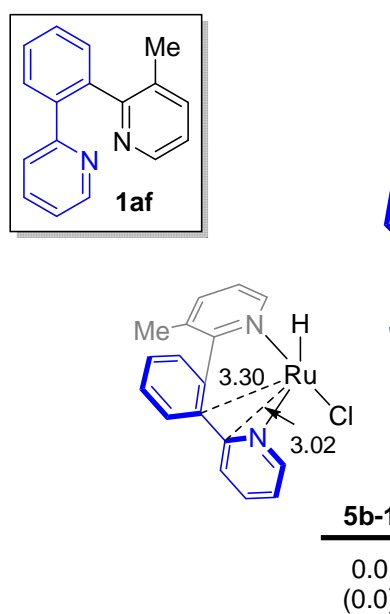

Figure S7.2.2 Computed barriers of the C(aryl)-C(aryl) oxidative addition in substrates 1a and 1af.

\subsubsection{Ru dichloride pathway (path a)}

The overall energy profile for " $\mathrm{RuCl}_{2}$ "-catalyzed the $\mathrm{C}($ aryl $)-\mathrm{C}($ aryl) bond activation of substrate $1 \mathrm{a}$ is shown in Figure S7.2.3. Although the oxidative addition of C(aryl)-C(aryl) (TS2a) with Ru dichloride species requires a low barrier of $20.8 \mathrm{kcal} / \mathrm{mol}$ with respect to the resting state $\mathbf{5 a}$, the formed $\mathrm{Ru}$ intermediate $\mathbf{6 a}$ is coordinatively saturated, which is difficult to react with $\mathrm{H}_{2}$ to generate $\mathrm{Ru}$ hydride. The hydride formation via pushing out one $\mathrm{Cl}$ ligand (TS5 and intermediate 11) and dissociation of one pyridine (intermediate 10) have unfavorable energetics. This indicates the " $\mathrm{RuCl}_{2}$ " pathway (path a in the main text) is disfavored. 


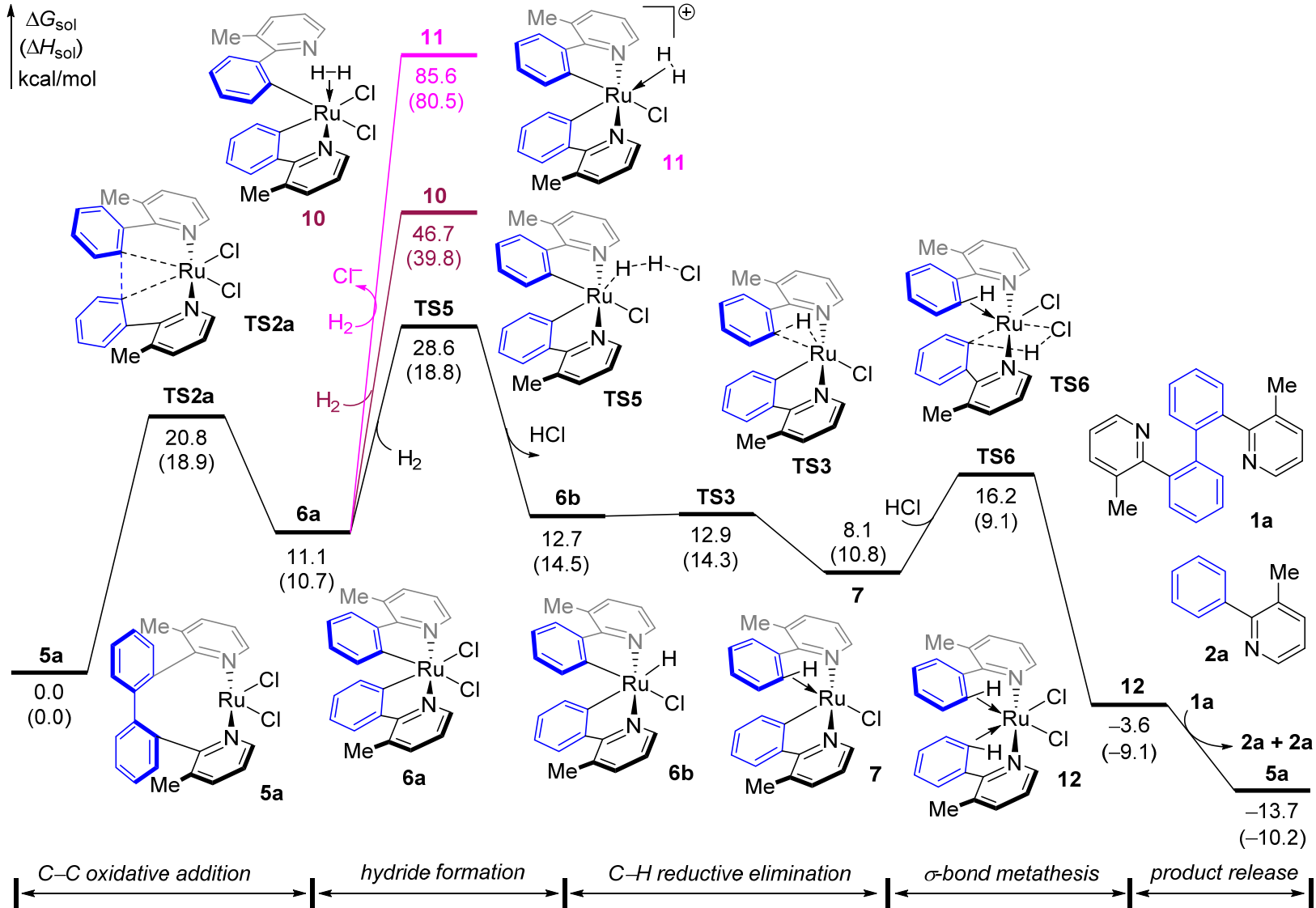

Figure S7.2.3 DFT-computed reaction energy profile of the $\mathrm{C}($ aryl $)-\mathrm{C}($ aryl) bond activation of substrate 1a catalyzed by the $\mathrm{RuCl}_{2}$ species.

\subsubsection{Ru dihydride pathway (path c)}

The overall energy profile for " $\mathrm{RuH}_{2}$ "-catalyzed the $\mathrm{C}(\operatorname{aryl})-\mathrm{C}(\operatorname{aryl})$ bond activation of substrate $\mathbf{1 a}$ is shown in Figure S7.2.4. The formation of the $\mathrm{Ru}$ dihydride 5c from 5a via double hydrogenation is endothermic by $34.8 \mathrm{kcal} / \mathrm{mol}$. This extremely unstable Ru hydride species results in a highly disfavored $\mathrm{C}(\operatorname{aryl})-\mathrm{C}($ aryl $)$ oxidative addition transition state $\operatorname{TS2c}\left(\Delta G^{\star}=53.0 \mathrm{kcal} / \mathrm{mol}\right.$ with respect to 5a). The subsequent hydrogenolysis process is also significantly less favorable than the RuHCl pathway. This indicates the " $\mathrm{RuH}_{2}$ " pathway (path $\mathrm{c}$ in the main text) is disfavored. 


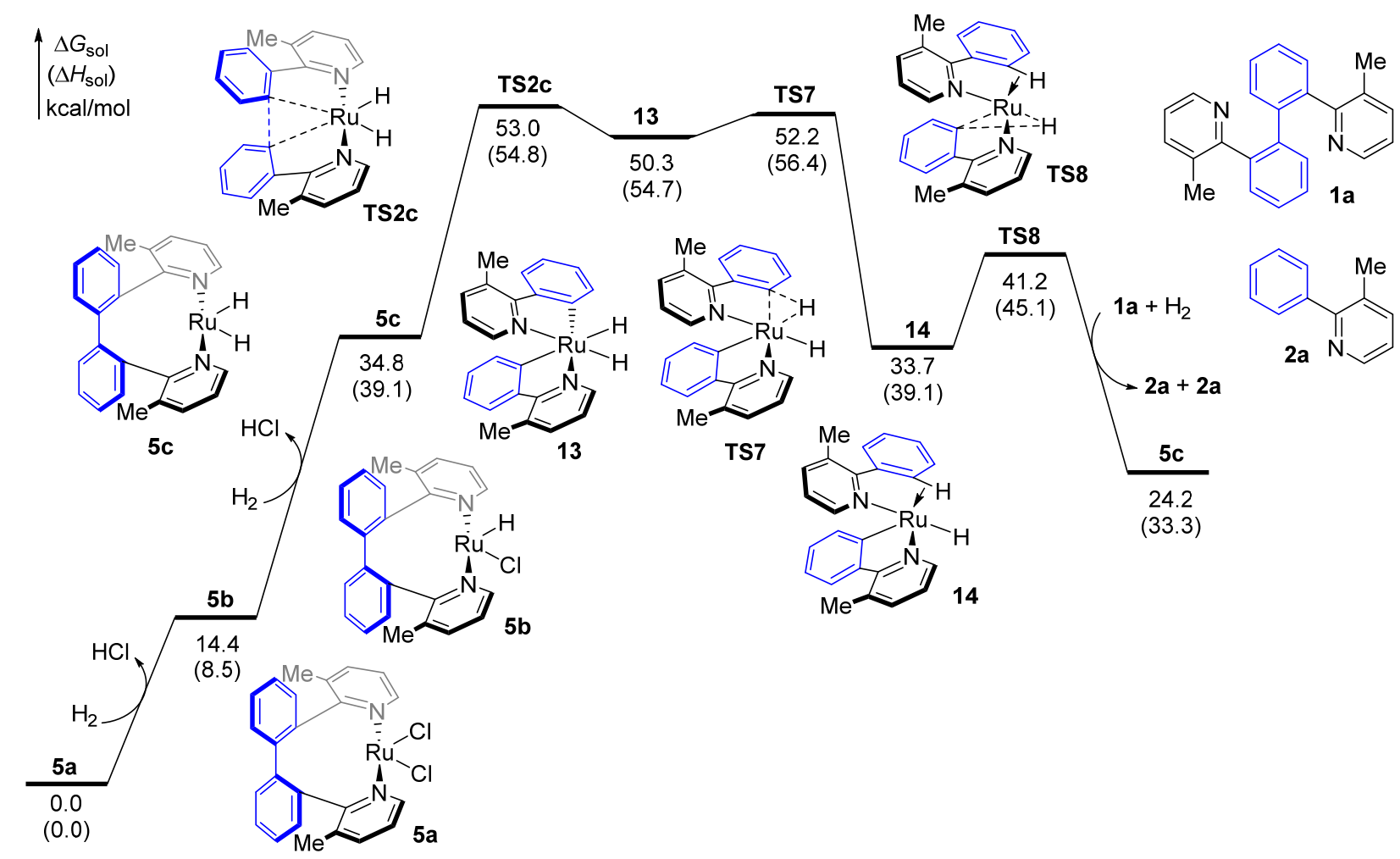

Figure S7.2.4. DFT-computed reaction energy profile of the $\mathrm{C}($ aryl $)-\mathrm{C}($ aryl) bond activation of substrate 1a catalyzed by the $\mathrm{RuH}_{2}$ species.

\subsubsection{Ru(0) pathway}

As shown in Figure $\mathrm{S} 7.2 .5$, we considered the formation of $\mathrm{Ru}(0)$ species via the reductive elimination of $\mathrm{Ru}$ dihydride 5c. Although the reductive elimination transition state from $\mathbf{5 c}$ cannot be computationally located, the calculation via scanning the $\mathrm{H} \cdots \mathrm{H}$ distance in $\mathbf{5 c}$ indicates the $\mathrm{H}-\mathrm{H}$ reductive elimination is a barrierless process. The formed $\mathrm{Ru}(0)$ species 15 are uphill in free energy by $45.2 \mathrm{kcal} / \mathrm{mol}$ with respect to $\mathbf{5 a}$. This leads to a dramatically high barrier of the subsequent $\mathrm{C}(\operatorname{aryl})-\mathrm{C}(\operatorname{aryl})$ oxidative addition $\left(\mathbf{T S 9}, \Delta G^{\ddagger}=50.8 \mathrm{kcal} / \mathrm{mol}\right)$. Thus, the $\mathrm{Ru}(0)$ pathway can be ruled out. 

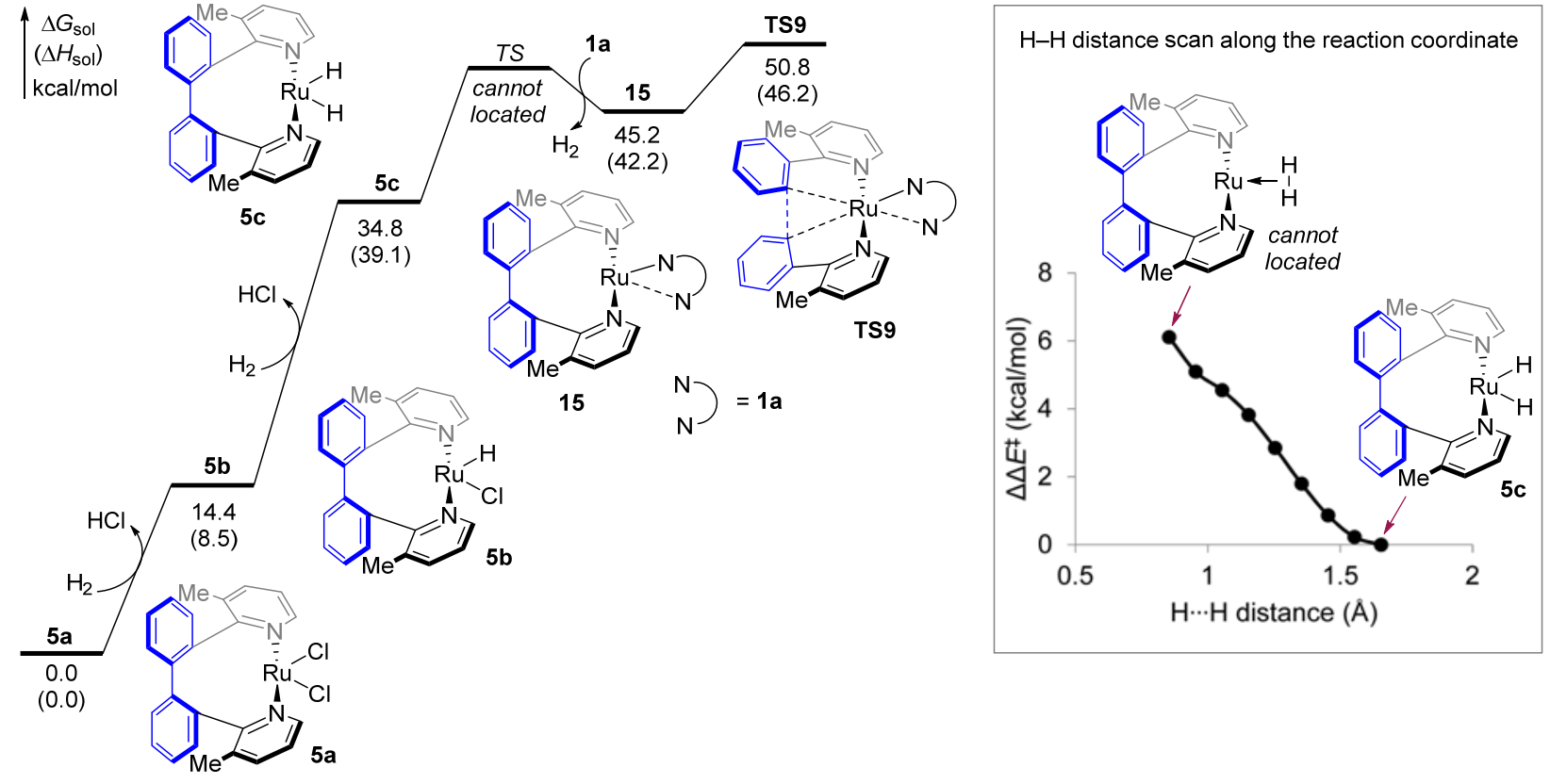

Figure S7.2.5. DFT-computed reaction energy profile of generating $\mathrm{Ru}(0)$ species and the $\mathrm{C}(\operatorname{aryl})-\mathrm{C}(\operatorname{aryl})$ bond activation of substrate $1 \mathbf{a}$ with the $\mathrm{Ru}(0)$ species. 


\subsection{Cartesian coordinates $(\AA)$ and energies of the optimized structures}

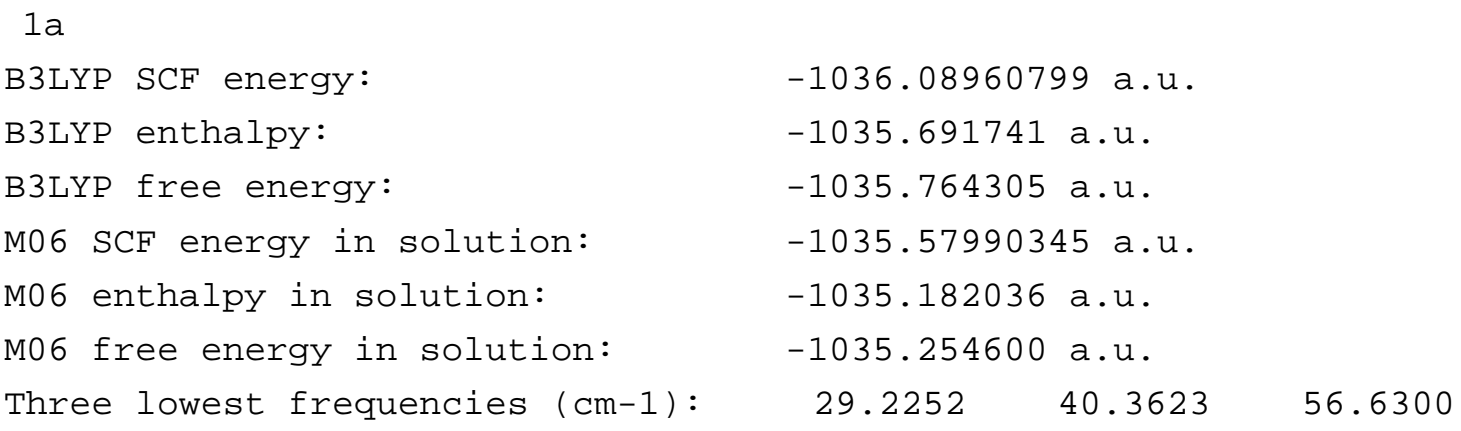

\section{Cartesian coordinates}

\begin{tabular}{|c|c|c|c|}
\hline АТОM & $x$ & Y & Z \\
\hline$N$ & 1.809256 & -1.671326 & ๑.278525 \\
\hline C & $\odot .656250$ & -1.582563 & -0.412077 \\
\hline C & $\odot .614683$ & -1.609189 & -1.824468 \\
\hline C & 1.843650 & -1.663410 & -2.493155 \\
\hline C & 3.037014 & -1.710225 & -1.781556 \\
\hline C & 2.961437 & -1.727901 & -0.389923 \\
\hline C & $-\odot .571470$ & -1.588919 & 0.451043 \\
\hline C & -1.595506 & -0.612657 & 0.430187 \\
\hline C & -2.716986 & -0.803737 & 1.262063 \\
\hline C & -2.827245 & -1.897116 & 2.115731 \\
\hline C & -1.794468 & -2.834564 & 2.165291 \\
\hline C & $-\odot .684510$ & -2.670424 & 1.342769 \\
\hline$N$ & 1.809255 & 1.671353 & $-\odot .278520$ \\
\hline C & $\odot .656245$ & 1.582568 & $\odot .412074$ \\
\hline C & $\odot .614670$ & 1.609180 & 1.824464 \\
\hline C & 1.843634 & 1.663397 & 2.493159 \\
\hline C & 3.037001 & 1.710234 & 1.781569 \\
\hline C & 2.961431 & 1.727931 & ๑. 389935 \\
\hline C & -0.571468 & 1.588917 & -0.451054 \\
\hline C & -1.595505 & $\odot .612656$ & -0.430192 \\
\hline C & -2.716991 & $\odot .803738$ & -1.262060 \\
\hline C & -2.827255 & 1.897117 & -2.115727 \\
\hline C & -1.794476 & 2.834562 & -2.165295 \\
\hline C & -0.684513 & 2.670422 & -1.342780 \\
\hline C & -0.664431 & 1.641691 & 2.626056 \\
\hline $\mathrm{H}$ & $-\odot .514751$ & 2.202276 & 3.555516 \\
\hline $\mathrm{H}$ & $-\odot .999032$ & $\odot .634378$ & 2.897399 \\
\hline $\mathrm{H}$ & -1.478570 & 2.115851 & 2.070653 \\
\hline C & $-\odot .664415$ & -1.641724 & -2.626064 \\
\hline $\mathrm{H}$ & -1.478532 & -2.115952 & -2.070686 \\
\hline $\mathrm{H}$ & $-\odot .514706$ & -2.202257 & -3.555551 \\
\hline $\mathrm{H}$ & -0.999065 & -0.634414 & -2.897357 \\
\hline
\end{tabular}




$\begin{array}{rrrr}\text { H } & 1.853765 & -1.689370 & -3.580746 \\ \text { H } & 3.996474 & -1.755789 & -2.288632 \\ \text { H } & 3.863452 & -1.797288 & 0.216749 \\ \text { H } & -1.854496 & -3.688582 & 2.834581 \\ \text { H } & -3.705562 & -2.006938 & 2.746362 \\ \text { H } & -3.500873 & -0.051452 & 1.250951 \\ \text { H } & 3.863449 & 1.797337 & -0.216731 \\ \text { H } & 3.996458 & 1.755793 & 2.288649 \\ \text { H } & 1.853741 & 1.689339 & 3.580752 \\ \text { H } & -1.854508 & 3.688581 & -2.834584 \\ \text { H } & -3.705574 & 2.006941 & -2.746354 \\ \text { H } & -3.500878 & 0.051452 & -1.250943 \\ \text { H } & 0.124486 & -3.393388 & 1.369898 \\ \text { H } & 0.124481 & 3.393387 & -1.369909\end{array}$

$2 a$

B3LYP SCF energy:

-518.64737402 a.u.

B3LYP enthalpy:

-518.437967 a.u.

B3LYP free energy:

-518.485445 a.u.

M06 SCF energy in solution:

-518.38312079 a.u.

M06 enthalpy in solution:

-518.173714 a.u.

M06 free energy in solution:

-518.221192 a.u.

$\begin{array}{llll}\text { Three lowest frequencies }(\mathrm{cm}-1): & 57.4466 & 86.1091 & 122.6870\end{array}$

Cartesian coordinates

\begin{tabular}{|c|c|c|c|}
\hline АTOM & $x$ & Y & Z \\
\hline$N$ & 1.175071 & -1.433229 & $-\odot .285666$ \\
\hline C & $\odot .677954$ & $-\odot .201444$ & -0.054666 \\
\hline C & 1.516874 & $\odot .914618$ & $\odot .187092$ \\
\hline C & 2.898377 & $\odot .695689$ & $\odot .125887$ \\
\hline C & 3.407891 & $-\odot .568803$ & $-\odot .150551$ \\
\hline C & 2.495593 & -1.606630 & -0.334136 \\
\hline C & $-\odot .813358$ & -0.133615 & -0.038928 \\
\hline C & -1.536249 & -1.189015 & $\odot .543228$ \\
\hline C & -2.928892 & -1.176273 & $\odot .561163$ \\
\hline C & -3.630488 & -0.116098 & -0.019422 \\
\hline C & -2.925934 & $\odot .926762$ & -0.621579 \\
\hline C & -1.529984 & $\odot .919173$ & -0.629172 \\
\hline C & 1.011421 & 2.293603 & $\odot .549792$ \\
\hline $\mathrm{H}$ & $\odot .082487$ & 2.250591 & 1.124846 \\
\hline $\mathrm{H}$ & $\odot .815074$ & 2.908555 & -0.337975 \\
\hline $\mathrm{H}$ & 1.760283 & 2.825060 & 1.146993 \\
\hline $\mathrm{H}$ & 2.838192 & -2.622593 & -0.526405 \\
\hline $\mathrm{H}$ & 4.477454 & $-\odot .749626$ & -0.202451 \\
\hline
\end{tabular}




$\begin{array}{rrrr}\text { H } & 3.574463 & 1.527238 & 0.313537 \\ \mathrm{H} & -3.461286 & 1.746091 & -1.094308 \\ \mathrm{H} & -4.717216 & -0.107496 & -0.008739 \\ \mathrm{H} & -3.469085 & -1.997183 & 1.025649 \\ \mathrm{H} & -0.998724 & 1.723450 & -1.127476 \\ \mathrm{H} & -0.986373 & -2.019289 & 0.973739\end{array}$

\section{$5 a$}

B3LYP SCF energy:

-2050.46941426 a.u.

B3LYP enthalpy:

-2050.061871 a.u.

B3LYP free energy:

-2050.144406 a.u.

M06 SCF energy in solution:

-2050.96405747 a.u.

M06 enthalpy in solution:

-2050.556514 a.u.

M06 free energy in solution:

-2050.639049 a.u.

Three lowest frequencies (cm-1): $\quad 39.8929 \quad 46.9987 \quad 51.5875$

Cartesian coordinates

\begin{tabular}{lrrr} 
ATOM & X & Y & \multicolumn{1}{l}{ Z } \\
Ru & $0.00000 \odot$ & $0.00000 \odot$ & 0.881494 \\
N & 0.143296 & 2.070754 & 0.704626 \\
C & -0.632014 & 2.440132 & -0.340122 \\
C & -0.731347 & 3.778642 & -0.747902 \\
C & 0.000000 & 4.719782 & -0.005445 \\
C & 0.775686 & 4.324868 & 1.079948 \\
C & 0.830854 & 2.972683 & 1.412242 \\
C & -1.379217 & 1.283064 & -0.943346 \\
C & -0.742844 & 0.084995 & -1.423253 \\
C & -1.568457 & -0.927032 & -1.976677 \\
C & -2.945543 & -0.802373 & -2.026650 \\
C & -3.562527 & 0.354787 & -1.529900 \\
C & -2.785864 & 1.374804 & -1.005809 \\
N & -0.143296 & -2.070754 & 0.704626 \\
C & 0.632014 & -2.440132 & -0.340122 \\
C & 0.731347 & -3.778642 & -0.747902 \\
C & 0.000000 & -4.719782 & -0.005445 \\
C & -0.775686 & -4.324868 & 1.079948 \\
C & -0.830854 & -2.972683 & 1.412242 \\
C & 1.379217 & -1.283064 & -0.943346 \\
C & 0.742844 & -0.084995 & -1.423253 \\
C & 1.568457 & 0.927032 & -1.976677 \\
C & 2.945543 & 0.802373 & -2.026650 \\
C & 3.562527 & -0.354787 & -1.529900 \\
C & 2.785864 & -1.374804 & -1.005809 \\
C & 1.563968 & -4.219263 & -1.929455
\end{tabular}




$\begin{array}{rrrr}\text { H } & 1.118187 & -5.099505 & -2.404579 \\ \mathrm{H} & 2.581262 & -4.496397 & -1.625289 \\ \mathrm{H} & 1.655802 & -3.431929 & -2.682794 \\ \mathrm{C} & -1.563968 & 4.219263 & -1.929455 \\ \mathrm{H} & -1.655802 & 3.431929 & -2.682794 \\ \mathrm{H} & -1.118187 & 5.099505 & -2.404579 \\ \mathrm{H} & -2.581262 & 4.496397 & -1.625289 \\ \mathrm{H} & -0.043583 & 5.768197 & -0.290848 \\ \mathrm{H} & 1.336846 & 5.048816 & 1.661803 \\ \mathrm{H} & 1.414899 & 2.576231 & 2.235687 \\ \mathrm{H} & -4.643924 & 0.451914 & -1.549377 \\ \mathrm{H} & -3.544921 & -1.600954 & -2.454844 \\ \mathrm{H} & -1.095089 & -1.815027 & -2.382691 \\ \mathrm{H} & -1.414899 & -2.576231 & 2.235687 \\ \mathrm{H} & -1.336846 & -5.048816 & 1.661803 \\ \mathrm{H} & 0.043583 & -5.768197 & -0.290848 \\ \mathrm{H} & 4.643924 & -0.451914 & -1.549377 \\ \mathrm{H} & 3.544921 & 1.600954 & -2.454844 \\ \mathrm{H} & 1.095089 & 1.815027 & -2.382691 \\ \mathrm{H} & -3.264204 & 2.260082 & -0.599667 \\ \mathrm{H} & 3.264204 & -2.260082 & -0.599667 \\ \mathrm{Cl} & -1.741885 & 0.180770 & 2.493268 \\ \mathrm{Cl} & 1.741885 & -0.180770 & 2.493268\end{array}$

5a-dimer

B3LYP SCF energy:

-4102.15746181 a.u.

B3LYP enthalpy:

-4101.322125 a.u.

B3LYP free energy:

-4101.466133 a.u.

M06 SCF energy in solution:

-4103.16092570 a.u.

M06 enthalpy in solution:

-4102.325589 a.u.

M06 free energy in solution:

-4102.469597 a.u.

Three lowest frequencies (cm-1): $\quad \begin{array}{llll}17.8461 & 18.8132 & 24.4744\end{array}$

Cartesian coordinates

$\begin{array}{lccc}\text { ATOM } & \text { X } & \text { Y } & \text { Z } \\ \text { Ru } & 1.538984 & -0.589364 & 0.310063 \\ \text { N } & 2.497745 & 1.129980 & 1.096061 \\ \text { C } & 3.125913 & 2.119341 & 0.406254 \\ \text { C } & 3.340706 & 3.397164 & 0.966194 \\ \text { C } & 2.949613 & 3.613598 & 2.289116 \\ \text { C } & 2.398800 & 2.568750 & 3.021835 \\ \text { C } & 2.189595 & 1.349378 & 2.395846 \\ \text { C } & 3.732690 & 1.802191 & -0.922422 \\ \text { C } & 4.887406 & 0.991605 & -0.949811\end{array}$




\begin{tabular}{|c|c|c|c|}
\hline C & 5.545743 & $\odot .784398$ & -2.168387 \\
\hline C & 5.070794 & 1.361111 & -3.346730 \\
\hline C & 3.922102 & 2.153028 & -3.317786 \\
\hline C & 3.263686 & 2.377208 & -2.109296 \\
\hline $\mathrm{N}$ & 3.080737 & -1.513301 & -0.832230 \\
\hline C & 4.355773 & -1.794983 & -0.448522 \\
\hline C & 5.146931 & -2.742320 & -1.139091 \\
\hline C & 4.617785 & -3.345347 & -2.280416 \\
\hline C & 3.355650 & -2.974337 & -2.728326 \\
\hline C & 2.626467 & -2.063015 & -1.982639 \\
\hline C & 5.013987 & -0.978925 & 0.617114 \\
\hline C & 5.370512 & $\odot .350894$ & 0.314997 \\
\hline C & 6.135166 & 1.080471 & 1.235811 \\
\hline C & 6.536595 & 0.510006 & 2.442899 \\
\hline C & 6.163442 & -0.800263 & 2.747985 \\
\hline C & 5.412143 & -1.539520 & 1.836804 \\
\hline C & 6.558279 & -3.075338 & -0.706840 \\
\hline $\mathrm{H}$ & 7.031272 & -3.736549 & -1.440028 \\
\hline $\mathrm{H}$ & 6.582170 & -3.582089 & $\odot .263617$ \\
\hline $\mathrm{H}$ & 7.176947 & -2.176661 & -0.607812 \\
\hline C & 4.022839 & 4.504245 & $\odot .192789$ \\
\hline $\mathrm{H}$ & 4.976324 & 4.174118 & $-\odot .233598$ \\
\hline $\mathrm{H}$ & 4.219816 & 5.358717 & 0.848177 \\
\hline $\mathrm{H}$ & 3.411378 & 4.860468 & -0.643718 \\
\hline $\mathrm{H}$ & 3.111907 & 4.586919 & 2.746003 \\
\hline $\mathrm{H}$ & 2.125029 & 2.687060 & 4.065374 \\
\hline $\mathrm{H}$ & $1.76654 \odot$ & $\odot .503157$ & 2.920248 \\
\hline $\mathrm{H}$ & 5.594879 & 1.189451 & -4.283358 \\
\hline $\mathrm{H}$ & 6.438168 & $\odot .164432$ & -2.188124 \\
\hline $\mathrm{H}$ & 2.927478 & -3.386617 & -3.636290 \\
\hline $\mathrm{H}$ & 5.210617 & -4.072577 & -2.829805 \\
\hline $\mathrm{H}$ & 6.452153 & -1.245949 & 3.695963 \\
\hline $\mathrm{H}$ & 7.131733 & 1.088754 & 3.144667 \\
\hline $\mathrm{H}$ & 6.414313 & 2.103781 & $\odot .998434$ \\
\hline $\mathrm{H}$ & 2.362311 & 2.981182 & -2.084129 \\
\hline $\mathrm{H}$ & 5.099124 & -2.549043 & 2.077969 \\
\hline $\mathrm{Cl}$ & 0.402713 & 0.620075 & -1.660762 \\
\hline $\mathrm{Cl}$ & -0.131216 & -2.389067 & -0.465246 \\
\hline $\mathrm{Ru}$ & -1.656620 & -0.438836 & -0.653778 \\
\hline$N$ & -2.671510 & 1.466722 & $-\odot .959750$ \\
\hline C & -3.391280 & 2.189688 & -0.064622 \\
\hline C & -3.699611 & 3.553468 & -0.281866 \\
\hline C & -3.294512 & 4.139526 & -1.481274 \\
\hline C & -2.627593 & 3.374337 & -2.430520 \\
\hline C & -2.333636 & 2.054534 & -2.128963 \\
\hline C & -3.992807 & 1.507135 & 1.119612 \\
\hline
\end{tabular}




\begin{tabular}{|c|c|c|c|}
\hline C & -5.070642 & 0.619720 & $\odot .920140$ \\
\hline C & -5.737860 & $\odot .099763$ & 2.037531 \\
\hline C & -5.338069 & $\odot .432109$ & 3.331446 \\
\hline C & -4.251198 & 1.285950 & 3.524308 \\
\hline C & -3.591511 & 1.826861 & 2.422459 \\
\hline $\mathrm{N}$ & -3.091903 & -1.671746 & 0.417479 \\
\hline C & -4.349966 & -1.997919 & ๑. 016779 \\
\hline C & -5.048802 & -3.095541 & $\odot .558235$ \\
\hline C & -4.435554 & -3.820662 & 1.583850 \\
\hline C & -3.188577 & -3.430858 & 2.052222 \\
\hline C & -2.545189 & -2.361406 & 1.443522 \\
\hline C & -5.049301 & -1.080120 & -0.932064 \\
\hline C & -5.455238 & 0.188358 & -0.462190 \\
\hline C & -6.194342 & 1.021377 & -1.310756 \\
\hline C & -6.520679 & 0.619893 & -2.607199 \\
\hline C & -6.108348 & -0.629462 & -3.072873 \\
\hline C & -5.384508 & -1.476859 & -2.232840 \\
\hline C & -6.439847 & -3.470375 & $\odot .095961$ \\
\hline $\mathrm{H}$ & -6.866991 & -4.229617 & $\odot .758350$ \\
\hline H & -6.439175 & -3.882118 & -0.919678 \\
\hline $\mathrm{H}$ & -7.114840 & -2.607780 & 0.086223 \\
\hline C & -4.490257 & 4.367563 & $\odot .718416$ \\
\hline H & -5.409375 & 3.856616 & 1.023718 \\
\hline H & -4.763102 & 5.335690 & $\odot .286961$ \\
\hline $\mathrm{H}$ & -3.918695 & 4.560558 & 1.632614 \\
\hline $\mathrm{H}$ & -3.528729 & 5.182934 & -1.676782 \\
\hline H & -2.324786 & 3.787082 & -3.387152 \\
\hline H & $-1.78470 \odot$ & 1.441146 & -2.828971 \\
\hline $\mathrm{H}$ & -3.916538 & 1.530611 & 4.528313 \\
\hline H & -5.868095 & $\odot .017824$ & 4.184764 \\
\hline $\mathrm{H}$ & -6.576017 & -0.575026 & 1.886319 \\
\hline H & -1.563039 & -2.046842 & 1.763734 \\
\hline $\mathrm{H}$ & -2.696292 & -3.949633 & 2.867854 \\
\hline $\mathrm{H}$ & -4.953281 & -4.668339 & 2.026033 \\
\hline $\mathrm{H}$ & -6.354656 & -0.947946 & -4.082011 \\
\hline $\mathrm{H}$ & -7.096625 & 1.280533 & -3.249497 \\
\hline H & -6.517017 & 1.993495 & $-\odot .947946$ \\
\hline H & -2.739639 & 2.483577 & 2.566813 \\
\hline $\mathrm{H}$ & -5.068378 & -2.453922 & -2.589319 \\
\hline $\mathrm{Cl}$ & -0.617929 & 0.317644 & 1.420983 \\
\hline $\mathrm{Cl}$ & 2.112223 & -1.954462 & 2.240304 \\
\hline H & 1.635013 & -1.761793 & -2.285010 \\
\hline $\mathrm{H}$ & 3.537570 & 2.594808 & -4.233128 \\
\hline $\mathrm{H}$ & -2.624187 & -0.979612 & -2.059260 \\
\hline $\mathrm{H}$ & -1.848296 & -1.051347 & -2.313904 \\
\hline
\end{tabular}


$5 a-1$

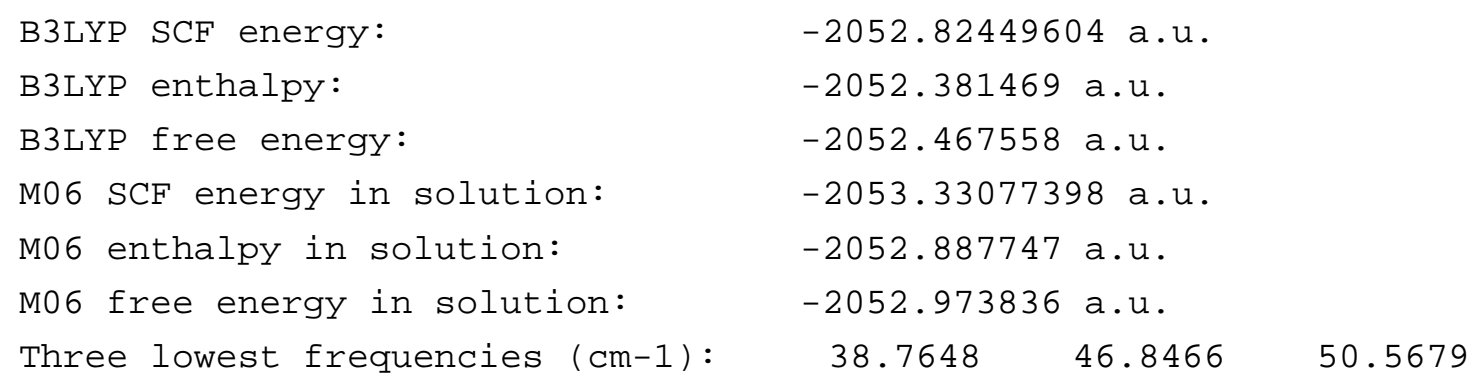

Cartesian coordinates

$\begin{array}{lrrr}\text { ATOM } & \text { X } & \text { Y } & \text { Z } \\ \text { C } & 2.371327 & -1.070937 & -1.466459 \\ \text { C } & 3.641584 & -0.581385 & -1.721399 \\ \text { C } & 4.161558 & 0.389010 & -0.872488 \\ \text { C } & 3.376648 & 0.899094 & 0.162149 \\ \text { C } & 2.075356 & 0.373684 & 0.340284 \\ \text { C } & 1.148762 & 1.070719 & 1.283236 \\ \text { C } & 1.350328 & 1.050228 & 2.668650 \\ \text { C } & 0.550083 & 1.815718 & 3.513935 \\ \text { C } & -0.454713 & 2.623402 & 2.978454 \\ \text { C } & -0.664104 & 2.647617 & 1.600290 \\ \text { C } & 0.126336 & 1.874584 & 0.738765 \\ \text { C } & -0.126520 & 1.875082 & -0.737524 \\ \text { C } & 0.663817 & 2.648802 & -1.598524 \\ \text { C } & 0.454388 & 2.625535 & -2.976701 \\ \text { C } & -0.550355 & 1.818139 & -3.512717 \\ \text { C } & -1.350503 & 1.051981 & -2.667945 \\ \text { C } & -1.148892 & 1.071511 & -1.282527 \\ \text { C } & -2.075388 & 0.373746 & -\odot .340034 \\ \text { C } & -3.376741 & 0.898883 & -\odot .161534 \\ \text { C } & -4.161563 & 0.388035 & 0.872795 \\ \text { C } & -3.641451 & -0.582841 & 1.721067 \\ \text { C } & -2.371137 & -1.072076 & 1.465776 \\ \text { N } & 1.614849 & -0.647969 & -\odot .428021 \\ \text { N } & -1.614741 & -0.648348 & 0.427597 \\ \text { Ru } & 0.000121 & -2.001001 & -0.000677 \\ \text { C } & 3.910851 & 2.020696 & 1.024616 \\ \text { H } & 4.872404 & 2.371445 & 0.637313 \\ \text { H } & 4.065055 & 1.701790 & 2.060843 \\ \text { H } & 3.222366 & 2.871621 & 1.055706 \\ \text { C } & -3.911093 & 2.020991 & -1.023249 \\ \text { H } & -4.872763 & 2.371254 & -0.635794 \\ \text { H } & -4.065111 & 1.702815 & -2.059726 \\ \text { H } & -3.222786 & 2.872088 & -1.053632\end{array}$




$\begin{array}{lrrr}\text { H } & 5.162842 & 0.781514 & -1.030695 \\ \text { H } & 4.205226 & -0.962884 & -2.566283 \\ \text { H } & 1.914477 & -1.816751 & -2.104928 \\ \text { H } & 2.115820 & 0.403617 & 3.082743 \\ \text { H } & 0.708459 & 1.776273 & 4.587879 \\ \text { H } & -1.075970 & 3.230619 & 3.631510 \\ \text { H } & -1.446038 & 3.274271 & 1.179792 \\ \text { H } & 1.445698 & 3.275243 & -1.177610 \\ \text { H } & 1.075565 & 3.233273 & -3.629345 \\ \text { H } & -0.708770 & 1.779440 & -4.586683 \\ \text { H } & -2.115957 & 0.405600 & -3.082470 \\ \text { H } & -5.162890 & 0.780321 & 1.031282 \\ \text { H } & -4.205030 & -0.964956 & 2.565714 \\ \text { H } & -1.914186 & -1.818242 & 2.103760 \\ \text { Cl } & -0.666381 & -2.185695 & -2.391473 \\ \text { Cl } & 0.666601 & -2.187377 & 2.389973 \\ \text { H } & -1.052423 & -3.355746 & 0.646235 \\ \text { H } & -1.347189 & -3.239144 & -0.087901 \\ \text { H } & 1.347492 & -3.238996 & 0.085547 \\ \text { H } & 1.052429 & -3.355317 & -0.648513\end{array}$

$5 a-2$

B3LYP SCF energy:

-2052.81765976 a.u.

B3LYP enthalpy:

-2052.374832 a.u.

B3LYP free energy:

-2052.460491 a.u.

M06 SCF energy in solution:

-2053.32745224 a.u.

M06 enthalpy in solution:

-2052.884624 a.u.

M06 free energy in solution:

-2052.970283 a.u.

$\begin{array}{lllll}\text { Three lowest frequencies }(\mathrm{cm}-1): & 36.4021 & 46.9430 & 49.7967\end{array}$

Cartesian coordinates

$\begin{array}{lccc}\text { ATOM } & \mathrm{X} & \mathrm{Y} & \mathrm{Z} \\ \mathrm{C} & 2.821340 & 0.295058 & -1.142818 \\ \mathrm{C} & 3.734222 & 1.337767 & -1.129106 \\ \mathrm{C} & 3.558749 & 2.364116 & -0.211735 \\ \mathrm{C} & 2.453017 & 2.348403 & 0.639044 \\ \mathrm{C} & 1.549577 & 1.263199 & 0.548748 \\ \text { C } & 0.293099 & 1.352225 & 1.353732 \\ \text { C } & 0.331454 & 1.227864 & 2.748434 \\ \text { C } & -0.801730 & 1.481213 & 3.518300 \\ \text { C } & -1.987553 & 1.879981 & 2.898817 \\ \text { C } & -2.040182 & 1.987116 & 1.510084 \\ \text { C } & -0.911694 & 1.717831 & 0.722116 \\ \text { C } & -1.023521 & 1.727038 & -0.771327\end{array}$




\begin{tabular}{|c|c|c|c|}
\hline C & $-\odot .523275$ & 2.778182 & -1.548491 \\
\hline C & $-\odot .627965$ & 2.755054 & -2.940367 \\
\hline C & -1.242154 & 1.675173 & -3.575945 \\
\hline C & -1.758337 & 0.626341 & -2.814099 \\
\hline C & -1.648761 & 0.637010 & -1.417700 \\
\hline C & -2.230982 & -0.445541 & -0.569458 \\
\hline C & -3.630431 & -0.585227 & -0.458988 \\
\hline C & -4.123722 & -1.489556 & 0.485547 \\
\hline C & -3.241645 & -2.191205 & 1.298929 \\
\hline C & -1.877385 & -2.019854 & 1.108721 \\
\hline $\mathrm{N}$ & 1.758843 & $\odot .231155$ & -0.309203 \\
\hline $\mathrm{N}$ & -1.370800 & -1.197027 & 0.166627 \\
\hline $\mathrm{Ru}$ & 0.747925 & -1.680382 & $-\odot .355490$ \\
\hline C & 2.232973 & 3.499747 & 1.595703 \\
\hline H & 2.958945 & 4.295405 & 1.401468 \\
\hline $\mathrm{H}$ & 2.348379 & 3.191262 & 2.640009 \\
\hline $\mathrm{H}$ & 1.227370 & 3.923194 & 1.501975 \\
\hline C & -4.589364 & $\odot .238933$ & -1.288687 \\
\hline $\mathrm{H}$ & -5.608560 & 0.138813 & -0.903123 \\
\hline H & -4.601859 & -0.078545 & -2.337728 \\
\hline $\mathrm{H}$ & -4.323954 & 1.301036 & -1.282645 \\
\hline H & 4.263073 & 3.190836 & -0.163386 \\
\hline $\mathrm{H}$ & 4.570571 & 1.323433 & -1.819882 \\
\hline $\mathrm{H}$ & 2.959885 & -0.550719 & -1.800437 \\
\hline $\mathrm{H}$ & 1.252661 & $\odot .906064$ & 3.221994 \\
\hline $\mathrm{H}$ & $-\odot .757092$ & 1.368385 & 4.597790 \\
\hline $\mathrm{H}$ & -2.871412 & 2.096838 & 3.492721 \\
\hline $\mathrm{H}$ & -2.966715 & 2.279788 & 1.023175 \\
\hline $\mathrm{H}$ & $-\odot .049377$ & 3.622424 & -1.055500 \\
\hline $\mathrm{H}$ & -0.232011 & 3.580156 & -3.525958 \\
\hline $\mathrm{H}$ & -1.322751 & 1.649184 & -4.658970 \\
\hline $\mathrm{H}$ & -2.237886 & -0.215915 & -3.306157 \\
\hline $\mathrm{H}$ & -5.197730 & -1.613563 & $\odot .599944$ \\
\hline $\mathrm{H}$ & -3.595475 & -2.868419 & 2.069474 \\
\hline $\mathrm{H}$ & -1.144255 & -2.536809 & 1.716958 \\
\hline $\mathrm{Cl}$ & 2.841660 & -2.812978 & -0.991730 \\
\hline $\mathrm{Cl}$ & 1.287329 & -1.979885 & 1.980694 \\
\hline $\mathrm{H}$ & 0.352037 & -3.425191 & -0.044560 \\
\hline $\mathrm{H}$ & $-\odot .072070$ & -3.282193 & -0.709536 \\
\hline $\mathrm{H}$ & $\odot .758819$ & -1.712444 & -2.170224 \\
\hline $\mathrm{H}$ & 0.177212 & -1.171624 & -2.016929 \\
\hline
\end{tabular}

5a-3

B3LYP SCF energy:

-2052.81069681 a.u. 
B3LYP enthalpy:

B3LYP free energy:

M06 SCF energy in solution:

M06 enthalpy in solution:

M06 free energy in solution:

Three lowest frequencies (cm-1):

$$
\begin{aligned}
& -2052.367816 \text { a.u. } \\
& -2052.454505 \text { a.u. } \\
& -2053.32297518 \text { a.u. } \\
& -2052.880094 \text { a.u. } \\
& -2052.966783 \text { a.u. }
\end{aligned}
$$

\begin{tabular}{|c|c|c|c|}
\hline ATOM & $x$ & Y & Z \\
\hline C & -2.659572 & $\odot .895775$ & -1.125780 \\
\hline C & -3.933086 & ๑. 359643 & -1.251359 \\
\hline C & -4.311059 & -0.669976 & -0.399844 \\
\hline C & -3.392224 & -1.180189 & $\odot .519676$ \\
\hline C & -2.101065 & -0.609632 & $\odot .550116$ \\
\hline C & -1.046479 & -1.254293 & 1.386975 \\
\hline C & -1.085893 & -1.191672 & 2.785427 \\
\hline C & -0.148225 & -1.879558 & 3.556106 \\
\hline C & 0.825452 & -2.657518 & 2.928460 \\
\hline C & 0.873353 & -2.722474 & 1.535221 \\
\hline C & -0.047232 & -2.019638 & 0.747956 \\
\hline C & $\odot .046242$ & -2.019682 & -0.747853 \\
\hline C & $-\odot .874617$ & -2.722161 & -1.535103 \\
\hline C & -0.826699 & -2.657258 & -2.928347 \\
\hline C & 0.147283 & -1.879705 & -3.556015 \\
\hline C & 1.085226 & -1.192172 & -2.785349 \\
\hline C & 1.045787 & -1.254731 & -1.386895 \\
\hline C & 2.100679 & -0.610508 & -0.550092 \\
\hline C & 3.391576 & -1.181663 & $-\odot .519771$ \\
\hline C & 4.310773 & -0.671883 & ๑. 399616 \\
\hline C & 3.933355 & $\odot .357927$ & 1.251152 \\
\hline C & 2.660094 & $\odot .894637$ & 1.125707 \\
\hline $\mathrm{N}$ & -1.754124 & 0.443138 & -0.231732 \\
\hline$N$ & 1.754264 & 0.442467 & $\odot .231758$ \\
\hline $\mathrm{Ru}$ & $\odot .00 \odot 461$ & 1.803533 & $\odot .000 \odot 82$ \\
\hline C & -3.781791 & -2.337090 & 1.413004 \\
\hline $\mathrm{H}$ & -4.751097 & -2.742418 & 1.106932 \\
\hline $\mathrm{H}$ & -3.867827 & -2.033525 & 2.462347 \\
\hline $\mathrm{H}$ & -3.045836 & -3.147380 & 1.378419 \\
\hline C & 3.780524 & -2.338753 & -1.413140 \\
\hline $\mathrm{H}$ & 4.749563 & -2.744665 & -1.106998 \\
\hline $\mathrm{H}$ & 3.866852 & -2.035156 & -2.462453 \\
\hline $\mathrm{H}$ & 3.044085 & -3.148602 & -1.378685 \\
\hline $\mathrm{H}$ & -5.309270 & -1.096822 & -0.456517 \\
\hline $\mathrm{H}$ & -4.613809 & $\odot .765951$ & -1.991928 \\
\hline $\mathrm{H}$ & -2.360509 & 1.740128 & -1.728441 \\
\hline $\mathrm{H}$ & -1.853506 & $-\odot .593435$ & 3.269382 \\
\hline
\end{tabular}

$\begin{array}{lll}28.4725 & 43.9799 & 46.3393\end{array}$

\section{Cartesian coordinates}




$\begin{array}{lrrr}\text { H } & -0.184360 & -1.814029 & 4.639860 \\ \mathrm{H} & 1.549358 & -3.210577 & 3.520505 \\ \mathrm{H} & 1.636584 & -3.323532 & 1.048956 \\ \mathrm{H} & -1.638095 & -3.322898 & -1.048824 \\ \mathrm{H} & -1.550832 & -3.210043 & -3.520370 \\ \mathrm{H} & 0.183454 & -1.814228 & -4.639771 \\ \mathrm{H} & 1.853090 & -0.594272 & -3.269323 \\ \mathrm{H} & 5.308788 & -1.099195 & 0.456186 \\ \mathrm{H} & 4.614325 & 0.763953 & 1.991650 \\ \mathrm{H} & 2.361533 & 1.739146 & 1.728393 \\ \mathrm{Cl} & -1.698317 & 3.561309 & -0.001184 \\ \mathrm{Cl} & 1.700060 & 3.560533 & 0.001032 \\ \mathrm{H} & -0.357475 & 1.550702 & 1.745730 \\ \mathrm{H} & 0.087894 & 2.224989 & 1.734743 \\ \mathrm{H} & -0.085262 & 2.225859 & -1.734524 \\ \mathrm{H} & 0.357016 & 1.549471 & -1.745686\end{array}$

$5 b$

B3LYP SCF energy:

-1590.82956449 a.u.

B3LYP enthalpy:

-1590.417571 a.u.

B3LYP free energy:

-1590.497465 a.u.

M06 SCF energy in solution:

-1591.31559931 a.u.

M06 enthalpy in solution:

M06 free energy in solution:

-1590.903606 a.u.

-1590.983500 a.u.

Three lowest frequencies (cm-1): $39.8092 \quad 47.5211 \quad 55.4280$

Cartesian coordinates

$\begin{array}{lcrc}\text { ATOM } & \mathrm{X} & \mathrm{Y} & \mathrm{Z} \\ \mathrm{Ru} & -0.103617 & -0.208580 & -1.055476 \\ \mathrm{~N} & -2.123631 & -0.641410 & -0.794315 \\ \mathrm{C} & -2.533274 & 0.354466 & 0.020525 \\ \mathrm{C} & -3.829232 & 0.406058 & 0.544837 \\ \mathrm{C} & -4.691775 & -0.643366 & 0.182639 \\ \mathrm{C} & -4.256146 & -1.664561 & -0.658240 \\ \mathrm{C} & -2.946427 & -1.639491 & -1.136253 \\ \mathrm{C} & -1.436554 & 1.372451 & 0.185359 \\ \mathrm{C} & -0.180627 & 1.135499 & 0.861866 \\ \mathrm{C} & 0.683908 & 2.260601 & 1.039267 \\ \mathrm{C} & 0.373952 & 3.516150 & 0.568076 \\ \mathrm{C} & -0.844024 & 3.735479 & -0.109252 \\ \mathrm{C} & -1.720763 & 2.688727 & -0.282483 \\ \mathrm{~N} & 1.948535 & 0.196537 & -0.924420 \\ \mathrm{C} & 2.471400 & -0.119498 & 0.291437 \\ \mathrm{C} & 3.830542 & 0.099979 & 0.582090\end{array}$




\begin{tabular}{|c|c|c|c|}
\hline C & 4.635570 & $\odot .613167$ & -0.445657 \\
\hline C & 4.095900 & $\odot .891293$ & -1.695975 \\
\hline C & 2.735811 & $\odot .674967$ & -1.896407 \\
\hline C & 1.497193 & -0.726692 & 1.258971 \\
\hline C & $\odot .205950$ & $-\odot .157097$ & 1.503681 \\
\hline C & $-\odot .666152$ & -0.827496 & 2.382141 \\
\hline C & -0.307378 & -2.026782 & 2.990318 \\
\hline C & $\odot .946080$ & -2.587963 & 2.740563 \\
\hline C & 1.831205 & -1.937882 & 1.883685 \\
\hline C & 4.441608 & -0.165026 & 1.940034 \\
\hline $\mathrm{H}$ & 5.271149 & $\odot .526445$ & 2.122165 \\
\hline $\mathrm{H}$ & 4.848194 & -1.181621 & 2.013167 \\
\hline $\mathrm{H}$ & 3.714094 & -0.049955 & 2.747893 \\
\hline C & -4.292490 & 1.517646 & 1.456014 \\
\hline $\mathrm{H}$ & -3.464056 & 1.946069 & 2.027337 \\
\hline $\mathrm{H}$ & -5.045301 & 1.151289 & 2.162045 \\
\hline $\mathrm{H}$ & -4.751007 & 2.337525 & $\odot .888380$ \\
\hline $\mathrm{H}$ & -5.707215 & -0.654902 & $\odot .571706$ \\
\hline $\mathrm{H}$ & -4.919098 & -2.476597 & $-\odot .939687$ \\
\hline H & -2.524707 & -2.395493 & -1.789986 \\
\hline $\mathrm{H}$ & -1.092494 & 4.723718 & -0.485605 \\
\hline H & 1.060557 & 4.341490 & 0.736207 \\
\hline H & 1.604115 & 2.112820 & 1.595647 \\
\hline $\mathrm{H}$ & 2.244173 & $\odot .874060$ & -2.840988 \\
\hline $\mathrm{H}$ & 4.708728 & 1.280172 & -2.502641 \\
\hline H & 5.689498 & 0.797243 & -0.251645 \\
\hline H & 1.229157 & -3.531558 & 3.197648 \\
\hline $\mathrm{H}$ & -1.005482 & -2.521024 & 3.660592 \\
\hline $\mathrm{H}$ & -1.635551 & -0.387105 & 2.593066 \\
\hline $\mathrm{H}$ & -2.663907 & 2.851477 & -0.796629 \\
\hline $\mathrm{H}$ & 2.789481 & -2.395280 & 1.659471 \\
\hline $\mathrm{Cl}$ & $\odot .323927$ & -2.136496 & -2.399036 \\
\hline $\mathrm{H}$ & -0.337902 & 0.639500 & -2.355868 \\
\hline
\end{tabular}

$5 b-1$

B3LYP SCF energy:

-1320.45441421 a.u.

B3LYP enthalpy:

B3LYP free energy:

-1320.156865 a.u.

-1320.223860 a.u.

M06 SCF energy in solution: $\quad-1321.07026471$ a.u.

M06 enthalpy in solution: $\quad-1320.772715 \mathrm{a} . \mathrm{u}$.

M06 free energy in solution: $\quad-1320.839710$ a.u.

Three lowest frequencies $(\mathrm{cm}-1): \quad 44.0962 \quad 48.3512 \quad 62.3798$

Cartesian coordinates 


\begin{tabular}{|c|c|c|c|}
\hline ATOM & $x$ & Y & Z \\
\hline $\mathrm{Ru}$ & 1.355172 & -0.806659 & -0.750172 \\
\hline$N$ & -0.511178 & -1.536506 & -0.106698 \\
\hline C & -1.640965 & $-\odot .793689$ & $\odot .060959$ \\
\hline C & -2.767872 & -1.299477 & $\odot .746418$ \\
\hline C & -2.728911 & -2.620838 & 1.198827 \\
\hline C & -1.597549 & -3.400020 & $\odot .965962$ \\
\hline C & $-\odot .515352$ & -2.818804 & $\odot .327314$ \\
\hline C & -1.676606 & $\odot .565136$ & -0.558233 \\
\hline C & $-\odot .817807$ & 1.614261 & -0.172158 \\
\hline C & -0.954752 & 2.869115 & -0.782363 \\
\hline C & -1.902020 & 3. 088183 & -1.782063 \\
\hline C & -2.736043 & 2.045680 & -2.180614 \\
\hline C & -2.623967 & $\odot .799898$ & -1.563521 \\
\hline C & $\odot .227097$ & 1.472348 & $\odot .879465$ \\
\hline C & $\odot .220781$ & 2.337715 & 1.979750 \\
\hline C & 1.230546 & 2.281870 & 2.935597 \\
\hline C & 2.253755 & 1.351178 & 2.755610 \\
\hline C & 2.217566 & $\odot .516607$ & 1.649133 \\
\hline C & -3.990774 & $-\odot .452556$ & 1.022760 \\
\hline $\mathrm{H}$ & -3.721992 & $\odot .576688$ & 1.276990 \\
\hline $\mathrm{H}$ & -4.562275 & -0.875091 & 1.855292 \\
\hline $\mathrm{H}$ & -4.660656 & -0.403166 & $\odot .156454$ \\
\hline $\mathrm{H}$ & -3.581200 & -3.031950 & 1.733239 \\
\hline $\mathrm{H}$ & -1.541661 & -4.433099 & 1.293425 \\
\hline $\mathrm{H}$ & $\odot .402501$ & -3.373292 & $\odot .153937$ \\
\hline $\mathrm{H}$ & -3.468685 & 2.195859 & -2.968444 \\
\hline $\mathrm{H}$ & -1.976248 & 4.064391 & -2.252577 \\
\hline $\mathrm{H}$ & -0.284720 & 3.671429 & $-\odot .487456$ \\
\hline $\mathrm{H}$ & 3.080381 & 1.269652 & 3.453639 \\
\hline $\mathrm{H}$ & 1.221776 & 2.949492 & 3.791675 \\
\hline $\mathrm{H}$ & -0.593882 & 3.048285 & 2.071499 \\
\hline $\mathrm{H}$ & -3.270782 & -0.014920 & -1.876400 \\
\hline $\mathrm{H}$ & 3.010567 & $-\odot .193929$ & 1.446317 \\
\hline $\mathrm{Cl}$ & 3.615945 & -0.501448 & -1.414383 \\
\hline $\mathrm{H}$ & 0.684661 & ๑.119839 & -1.807796 \\
\hline $\mathrm{N}$ & 1.222725 & $\odot .551899$ & 0.723158 \\
\hline
\end{tabular}

$5 \mathrm{C}$

B3LYP SCF energy:

-1131.17531456 a.u.

B3LYP enthalpy:

-1130.759256 a.u.

B3LYP free energy:

-1130.835819 a.u.

M06 SCF energy in solution:

-1131.65114364 a.u.

Mo6 enthalpy in solution:

-1131.235085 a.u. 
M06 free energy in solution:

Three lowest frequencies (cm-1):
-1131.311648 a.u.

42.3522

45.5127

56.1780

Cartesian coordinates

\begin{tabular}{|c|c|c|c|}
\hline АTOM & $x$ & Y & Z \\
\hline $\mathrm{Ru}$ & -0.000035 & $\odot .000973$ & -1.322679 \\
\hline $\mathrm{N}$ & 2.026811 & 0.379579 & -1.114674 \\
\hline C & 2.486408 & -0.305395 & -0.032889 \\
\hline C & 3.810542 & -0.190089 & $\odot .415429$ \\
\hline C & 4.664300 & 0.654949 & -0.312063 \\
\hline C & 4.189961 & 1.340878 & -1.426043 \\
\hline C & 2.858168 & 1.182716 & -1.798330 \\
\hline C & 1.449485 & $-1.20 \odot 170$ & $\odot .587395$ \\
\hline C & 0.186576 & -0.724864 & 1.077848 \\
\hline C & -0.701245 & -1.673300 & 1.637194 \\
\hline C & -0.387690 & -3.022478 & 1.711291 \\
\hline C & 0.844175 & -3.481743 & 1.230321 \\
\hline C & 1.740406 & -2.576530 & $\odot .679568$ \\
\hline $\mathrm{N}$ & -2.026947 & -0.378363 & -1.115193 \\
\hline C & -2.486560 & 0.305339 & -0.032697 \\
\hline C & -3.810610 & $\odot .189203$ & $\odot .415716$ \\
\hline C & -4.664144 & -0.655390 & -0.312518 \\
\hline C & -4.189766 & -1.340030 & -1.427305 \\
\hline C & -2.858094 & -1.181078 & -1.799634 \\
\hline C & -1.449582 & 1.199545 & $\odot .588353$ \\
\hline C & -0.186709 & 0.723814 & 1.078496 \\
\hline C & 0.701280 & 1.671850 & 1.638283 \\
\hline C & 0.387839 & 3.020978 & 1.713315 \\
\hline C & -0.844035 & 3.480666 & 1.232703 \\
\hline C & -1.740345 & 2.575914 & ๑. 681393 \\
\hline C & -4.326453 & $\odot .915108$ & 1.637437 \\
\hline $\mathrm{H}$ & -5.124390 & $\odot .337487$ & 2.116308 \\
\hline $\mathrm{H}$ & -4.747121 & 1.895606 & 1.379939 \\
\hline $\mathrm{H}$ & -3.537087 & 1.088665 & 2.374023 \\
\hline C & 4.326367 & -0.917393 & 1.636348 \\
\hline $\mathrm{H}$ & 3.537169 & -1.090916 & 2.373124 \\
\hline $\mathrm{H}$ & 5.124923 & $-\odot .340726$ & 2.115331 \\
\hline $\mathrm{H}$ & 4.746196 & -1.898012 & 1.377953 \\
\hline $\mathrm{H}$ & 5.696509 & 0.774432 & $\odot .0 \odot 7798$ \\
\hline $\mathrm{H}$ & 4.837297 & 1.997103 & -1.999417 \\
\hline $\mathrm{H}$ & 2.423095 & 1.687388 & -2.653123 \\
\hline $\mathrm{H}$ & 1.096204 & -4.537336 & 1.277180 \\
\hline $\mathrm{H}$ & -1.097137 & -3.717198 & 2.153041 \\
\hline $\mathrm{H}$ & -1.646902 & -1.323258 & 2.038571 \\
\hline $\mathrm{H}$ & -2.422940 & -1.684613 & -2.655053 \\
\hline $\mathrm{H}$ & -4.837036 & -1.995881 & -2.001182 \\
\hline
\end{tabular}




$\begin{array}{rrrr}\text { H } & -5.696258 & -0.775614 & 0.007383 \\ \text { H } & -1.095952 & 4.536256 & 1.280267 \\ \text { H } & 1.097388 & 3.715349 & 2.155446 \\ \text { H } & 1.646965 & 1.321475 & 2.039305 \\ \text { H } & 2.687912 & -2.931233 & 0.284334 \\ \text { H } & -2.687824 & 2.930939 & 0.286385 \\ \text { H } & 0.255593 & -1.001215 & -2.534814 \\ \text { H } & -0.255738 & 1.004960 & -2.533541\end{array}$

$6 a$

B3LYP SCF energy:

B3LYP enthalpy:

B3LYP free energy:

M06 SCF energy in solution:

M06 enthalpy in solution:

M06 free energy in solution:

Three lowest frequencies (cm-1):

$$
\begin{aligned}
& -2050.45426918 \text { a.u. } \\
& -2050.046152 \text { a.u. } \\
& -2050.128050 \text { a.u. } \\
& -2050.94758441 \text { a.u. } \\
& -2050.539467 \text { a.u. } \\
& -2050.621365 \text { a.u. } \\
& \begin{array}{ll}
21.4075 & 22.2415
\end{array}
\end{aligned}
$$

41.0564
Cartesian coordinates

\begin{tabular}{lrrr} 
ATOM & X & Y & \multicolumn{1}{l}{ Z } \\
Ru & $0.00000 \odot$ & 0.000000 & 0.825945 \\
N & 0.368158 & 2.088984 & 0.880798 \\
C & -0.184325 & 2.877793 & -0.091715 \\
C & 0.000000 & 4.285588 & -0.039194 \\
C & 0.718305 & 4.810564 & 1.037389 \\
C & 1.240272 & 3.989137 & 2.030218 \\
C & 1.045056 & 2.623528 & 1.910157 \\
C & -0.949654 & 2.096241 & -1.075032 \\
C & -1.010243 & 0.704846 & -0.836769 \\
C & -1.783267 & -0.119165 & -1.654778 \\
C & -2.489033 & 0.414172 & -2.730931 \\
C & -2.410304 & 1.781811 & -3.004246 \\
C & -1.654063 & 2.611937 & -2.185879 \\
N & -0.368158 & -2.088984 & 0.880798 \\
C & 0.184325 & -2.877793 & -0.091715 \\
C & 0.000000 & -4.285588 & -0.039194 \\
C & -0.718305 & -4.810564 & 1.037389 \\
C & -1.240272 & -3.989137 & 2.030218 \\
C & -1.045056 & -2.623528 & 1.910157 \\
C & 0.949654 & -2.096241 & -1.075032 \\
C & 1.010243 & -0.704846 & -0.836769 \\
C & 1.783267 & 0.119165 & -1.654778 \\
C & 2.489033 & -0.414172 & -2.730931 \\
C & 2.410304 & -1.781811 & -3.004246
\end{tabular}




$\begin{array}{lrrr}\mathrm{C} & 1.654063 & -2.611937 & -2.185879 \\ \mathrm{C} & 0.536520 & -5.264606 & -1.057729 \\ \mathrm{H} & 0.232747 & -6.280548 & -0.788565 \\ \mathrm{H} & 1.631230 & -5.251410 & -1.104570 \\ \mathrm{H} & 0.156777 & -5.065988 & -2.066115 \\ \mathrm{C} & -0.536520 & 5.264606 & -1.057729 \\ \mathrm{H} & -0.156777 & 5.065988 & -2.066115 \\ \mathrm{H} & -0.232747 & 6.280548 & -0.788565 \\ \mathrm{H} & -1.631230 & 5.251410 & -1.104570 \\ \mathrm{H} & -.861719 & 5.886552 & 1.093914 \\ \mathrm{H} & 1.792484 & 4.390157 & 2.873043 \\ \mathrm{H} & 1.448484 & 1.920390 & 2.626811 \\ \mathrm{H} & -2.944394 & 2.204946 & -3.850344 \\ \mathrm{H} & -3.095013 & -0.236818 & -3.356234 \\ \mathrm{H} & -1.838232 & -1.183666 & -1.453361 \\ \mathrm{H} & -1.448484 & -1.920390 & 2.626811 \\ \mathrm{H} & -1.792484 & -4.390157 & 2.873043 \\ \mathrm{H} & -0.861719 & -5.886552 & 1.093914 \\ \mathrm{H} & 2.944394 & -2.204946 & -3.850344 \\ \mathrm{H} & 3.095013 & 0.236818 & -3.356234 \\ \mathrm{H} & 1.838232 & 1.183666 & -1.453361 \\ \mathrm{H} & -1.627919 & 3.663977 & -2.421914 \\ \mathrm{H} & 1.627919 & -3.663977 & -2.421914 \\ \mathrm{Cl} & -2.165142 & 0.381367 & 1.782471 \\ \mathrm{Cl} & 2.165142 & -0.381367 & 1.782471\end{array}$

$6 b$

B3LYP SCF energy:

-1590.83181612 a.u.

-1590.418739 a.u.

B3LYP enthalpy:

-1590.497673 a.u.

B3LYP free energy:

-1591.31698553 a.u.

M06 SCF energy in solution:

- 1590.903908 a.u.

M๑6 free energy in solution:

-1590.982842 a.u.

Three lowest frequencies (cm-1): $\quad 26.2921 \quad 31.3728 \quad 42.9811$

Cartesian coordinates

$\begin{array}{lccc}\text { ATOM } & \mathrm{X} & \mathrm{Y} & \mathrm{Z} \\ \mathrm{Ru} & 0.007529 & -0.899116 & -0.106348 \\ \mathrm{~N} & 2.109641 & -0.834953 & -0.453861 \\ \mathrm{C} & 2.832442 & 0.136594 & -.179218 \\ \mathrm{C} & 4.200671 & -.324167 & -0.137322 \\ \mathrm{C} & 4.781228 & -0.554451 & -1.056827 \\ \mathrm{C} & 4.041697 & -1.577520 & -1.640376 \\ \mathrm{C} & 2.696167 & -1.678049 & -1.318350\end{array}$




\begin{tabular}{|c|c|c|c|}
\hline C & 2.012952 & ๑. 886281 & 1.146954 \\
\hline C & 0.615757 & ๑. 651848 & 1.065191 \\
\hline C & -0.240026 & 1.271529 & 1.982475 \\
\hline C & $\odot .267891$ & 2.083337 & 2.999991 \\
\hline C & 1.642516 & 2.298406 & 3.096246 \\
\hline C & 2.507887 & 1.705290 & 2.178512 \\
\hline $\mathrm{N}$ & -2.070392 & -0.930759 & $\odot .262599$ \\
\hline C & -2.837202 & $\odot .101202$ & -0.215456 \\
\hline C & -4.214233 & $\odot .164830$ & $\odot .128948$ \\
\hline C & -4.737704 & -0.854192 & 0.929472 \\
\hline C & -3.943336 & -1.902676 & 1.380690 \\
\hline C & -2.604940 & -1.902444 & 1.023901 \\
\hline C & -2.056088 & 1.049563 & -1.026885 \\
\hline C & $-\odot .653650$ & $\odot .823622$ & -1.058361 \\
\hline C & $\odot .155795$ & 1.692079 & -1.802699 \\
\hline C & $-\odot .387323$ & 2.755405 & -2.520210 \\
\hline C & -1.768454 & 2.964257 & -2.514381 \\
\hline C & -2.590730 & 2.118964 & -1.778284 \\
\hline C & -5.155635 & 1.273850 & -0.282156 \\
\hline H & -6.111836 & 1.157449 & $\odot .236856$ \\
\hline $\mathrm{H}$ & -5.367707 & 1.261867 & -1.357807 \\
\hline H & -4.760814 & 2.264477 & -0.033251 \\
\hline C & 5.060483 & 1.432435 & $\odot .425556$ \\
\hline $\mathrm{H}$ & 4.543439 & 2.397092 & 0.420378 \\
\hline $\mathrm{H}$ & 5.970514 & 1.535569 & -0.173469 \\
\hline $\mathrm{H}$ & 5.375362 & 1.231018 & 1.457031 \\
\hline H & 5.828709 & $-\odot .423894$ & -1.317168 \\
\hline $\mathrm{H}$ & 4.486636 & -2.271533 & -2.345319 \\
\hline H & 2.054329 & -2.443064 & -1.736214 \\
\hline H & 2.046704 & 2.922502 & 3.888483 \\
\hline H & $-\odot .410301$ & 2.549868 & 3.710470 \\
\hline $\mathrm{H}$ & -1.312625 & 1.124828 & 1.906435 \\
\hline H & -1.925525 & -2.692610 & 1.318944 \\
\hline $\mathrm{H}$ & -4.344828 & -2.700491 & 1.996073 \\
\hline $\mathrm{H}$ & -5.788995 & -0.814357 & 1.203320 \\
\hline H & -2.206418 & 3.777436 & -3.086467 \\
\hline H & $\odot .263659$ & 3.414991 & -3.089488 \\
\hline H & 1.229622 & 1.530397 & -1.825261 \\
\hline $\mathrm{H}$ & 3.570337 & 1.859013 & 2.304782 \\
\hline H & -3.655139 & 2.292149 & -1.817914 \\
\hline $\mathrm{Cl}$ & $\odot .122010$ & -3.336636 & -0.064411 \\
\hline $\mathrm{H}$ & $\odot .077670$ & $-\odot .914778$ & -1.662331 \\
\hline
\end{tabular}


B3LYP SCF energy:

B3LYP enthalpy:

B3LYP free energy:

M06 SCF energy in solution:

M06 enthalpy in solution:

M06 free energy in solution:

Three lowest frequencies (cm-1):

$$
\begin{aligned}
& -1590.84315090 \text { a.u. } \\
& -1590.428241 \text { a.u. } \\
& -1590.508674 \text { a.u. } \\
& -1591.32470649 \text { a.u. } \\
& -1590.909797 \text { a.u. } \\
& -1590.990230 \text { a.u. }
\end{aligned}
$$

\begin{tabular}{|c|c|c|c|}
\hline ATOM & $x$ & $Y$ & Z \\
\hline $\mathrm{Ru}$ & -0.011356 & 0.942052 & -0.210087 \\
\hline $\mathrm{N}$ & -2.013992 & 0.840055 & -0.775597 \\
\hline C & -2.818534 & -0.038402 & -0.097553 \\
\hline C & -4.184149 & -0.157824 & -0.472525 \\
\hline C & -4.657328 & $\odot .656162$ & -1.504898 \\
\hline C & -3.825220 & 1.565483 & -2.152347 \\
\hline C & -2.501387 & 1.622552 & -1.749592 \\
\hline C & -2.085705 & -0.748678 & $\odot .965230$ \\
\hline C & $-\odot .699406$ & -0.428728 & 1.059440 \\
\hline C & $\odot .069430$ & -1.023785 & 2.068240 \\
\hline C & -0.497944 & -1.920976 & 2.972374 \\
\hline C & -1.854914 & -2.240761 & 2.881311 \\
\hline C & -2.639425 & -1.658528 & 1.890626 \\
\hline $\mathrm{N}$ & 2.097635 & 1.006683 & $\odot .195989$ \\
\hline C & 2.893801 & $-\odot .0 \odot 6187$ & -0.267288 \\
\hline C & 4.301627 & 0.117302 & $-\odot .273046$ \\
\hline C & 4.855119 & 1.276964 & $\odot .281032$ \\
\hline C & 4.037791 & 2.269070 & $\odot .810323$ \\
\hline C & 2.661251 & 2.105228 & 0.733444 \\
\hline C & 2.151149 & -1.189422 & -0.761666 \\
\hline C & 0.859615 & -1.006292 & -1.303406 \\
\hline C & 0.101205 & -2.099126 & -1.733854 \\
\hline C & $\odot .591201$ & -3.395417 & -1.586716 \\
\hline C & 1.845891 & -3.593944 & -1.007403 \\
\hline C & 2.615163 & -2.503533 & -0.597885 \\
\hline C & 5.236932 & -0.902986 & -0.884717 \\
\hline $\mathrm{H}$ & 6.155882 & -0.409764 & -1.217884 \\
\hline $\mathrm{H}$ & 4.790560 & -1.409150 & -1.744680 \\
\hline $\mathrm{H}$ & 5.533172 & -1.676672 & -0.165118 \\
\hline C & -5.166250 & -1.110479 & $\odot .167917$ \\
\hline $\mathrm{H}$ & -4.821022 & -2.149363 & $\odot .125970$ \\
\hline $\mathrm{H}$ & -6.127450 & -1.059285 & -0.352652 \\
\hline $\mathrm{H}$ & -5.350220 & $-\odot .865456$ & 1.220334 \\
\hline $\mathrm{H}$ & -5.700923 & 0.574245 & -1.798196 \\
\hline $\mathrm{H}$ & -4.189657 & 2.210945 & -2.944270 \\
\hline $\mathrm{H}$ & -1.792382 & 2.308420 & -2.202111 \\
\hline
\end{tabular}

19.9816
35.0507
Cartesian coordinates 


$\begin{array}{lrrr}\text { H } & -2.305742 & -2.935383 & 3.584961 \\ \text { H } & 0.116687 & -2.367439 & 3.751302 \\ \text { H } & 1.122712 & -0.773980 & 2.152711 \\ \text { H } & 1.962720 & 2.851297 & 1.097015 \\ \text { H } & 4.451588 & 3.169447 & 1.252638 \\ \text { H } & 5.935560 & 1.398664 & 0.282133 \\ \text { H } & 2.227559 & -4.600958 & -0.863760 \\ \text { H } & -0.004593 & -4.245353 & -1.907567 \\ \text { H } & -0.879432 & -1.926606 & -2.166896 \\ \text { H } & -3.687773 & -1.915459 & 1.864087 \\ \text { H } & 3.562295 & -2.687464 & -0.104984 \\ \text { Cl } & -0.551394 & 2.859007 & 1.090933 \\ \text { H } & 0.548856 & 0.002603 & -1.730066\end{array}$

8

B3LYP SCF energy:

-1592.03484302 a.u.

B3LYP enthalpy:

-1591.602357 a.u.

B3LYP free energy:

-1591.686128 a.u.

M06 SCF energy in solution:

-1592.52209986 a.u.

M06 enthalpy in solution:

-1592.089614 a.u.

M06 free energy in solution:

-1592.173385 a.u.

Three lowest frequencies (cm-1): $21.9660 \quad 26.3593 \quad 35.2016$

Cartesian coordinates

\begin{tabular}{lccc} 
ATOM & X & Y & \multicolumn{1}{l}{ Z } \\
Ru & -0.080346 & 0.030035 & -0.037616 \\
N & -1.843308 & 1.164087 & -0.052653 \\
C & -3.018882 & 0.467315 & -0.106834 \\
C & -4.238137 & 1.137411 & 0.178122 \\
C & -4.188165 & 2.509944 & 0.432006 \\
C & -2.982679 & 3.206276 & 0.421078 \\
C & -1.825031 & 2.481147 & 0.191337 \\
C & -2.807619 & -0.940476 & -0.483872 \\
C & -1.441983 & -1.341029 & -0.576645 \\
C & -1.158321 & -2.630326 & -1.052057 \\
C & -2.175456 & -3.525226 & -1.387111 \\
C & -3.511788 & -3.138156 & -1.272527 \\
C & -3.821784 & -1.853523 & -0.835446 \\
N & 1.724750 & -1.115557 & 0.252879 \\
C & 2.934831 & -0.540507 & 0.037933 \\
C & 4.140749 & -1.240075 & 0.245282 \\
C & 4.050855 & -2.557115 & 0.706919 \\
C & 2.808355 & -3.125013 & 0.970045 \\
C & 1.666937 & -2.367808 & 0.742068
\end{tabular}




$\begin{array}{lrrr}\text { C } & 2.900075 & 0.902615 & -0.337605 \\ \text { C } & 2.262376 & 1.811830 & 0.527872 \\ \text { C } & 2.217995 & 3.172057 & 0.210530 \\ \text { C } & 2.797263 & 3.638427 & -0.971142 \\ \text { C } & 3.428879 & 2.739786 & -1.834795 \\ \text { C } & 3.486145 & 1.382015 & -1.518107 \\ \text { C } & 5.495312 & -0.604729 & 0.028706 \\ \text { H } & 6.268077 & -1.166825 & 0.562256 \\ \text { H } & 5.519235 & 0.430416 & 0.381729 \\ \text { H } & 5.776094 & -0.590296 & -1.031542 \\ \text { C } & -5.584865 & 0.457622 & 0.263909 \\ \text { H } & -5.968328 & 0.164916 & -0.721301 \\ \text { H } & -6.317146 & 1.139914 & 0.706645 \\ \text { H } & -5.547676 & -0.442190 & 0.886237 \\ \text { H } & -5.114507 & 3.034956 & 0.651641 \\ \text { H } & -2.937085 & 4.272915 & 0.613207 \\ \text { H } & -\odot .843448 & 2.942322 & 0.210693 \\ \text { H } & -4.310537 & -3.824829 & -1.539294 \\ \text { H } & -1.924009 & -4.520287 & -1.748499 \\ \text { H } & -0.125198 & -2.941202 & -1.179888 \\ \text { H } & 0.674751 & -2.745713 & 0.950466 \\ \text { H } & 2.718695 & -4.133665 & 1.359834 \\ \text { H } & 4.961516 & -3.122781 & 0.887807 \\ \text { H } & 3.877418 & 3.096113 & -2.758194 \\ \text { H } & 2.761420 & 4.696141 & -1.217193 \\ \text { H } & 1.737935 & 3.865587 & 0.895912 \\ \text { H } & -4.863722 & -1.571623 & -0.794215 \\ \text { H } & 3.968756 & 0.687993 & -2.200406 \\ \text { Cl } & -0.403843 & -0.189702 & 2.341069 \\ \text { H } & 1.827586 & 1.452808 & 1.458762 \\ \text { H } & 0.266832 & -0.125812 & -1.757638 \\ & 0.213449 & 0.710043 & -1.639668\end{array}$

9

B3LYP SCF energy:

-1592.03602639 a.u.

B3LYP enthalpy:

-1591.601618 a.u.

B3LYP free energy:

M06 SCF energy in solution:

-1591.686045 a.u.

M06 enthalpy in solution:

M06 free energy in solution:

-1592.51300555 a.u.

-1592.078597 a.u.

-1592.163024 a.u.

Three lowest frequencies $(\mathrm{cm}-1): \quad 17.3930 \quad 27.4178 \quad 28.9489$

Cartesian coordinates

$\begin{array}{llll}\text { ATOM } & X & Y & Z\end{array}$ 


\begin{tabular}{|c|c|c|c|}
\hline $\mathrm{Ru}$ & $\odot .237613$ & $\odot .566052$ & -0.633667 \\
\hline $\mathrm{N}$ & 2.258680 & 0.954553 & -0.203136 \\
\hline C & 3.047713 & -0.096524 & 0.175321 \\
\hline C & 4.350591 & $\odot .120903$ & 0.679123 \\
\hline C & 4.823292 & 1.436728 & 0.708408 \\
\hline C & 4.030342 & 2.488442 & $\odot .259501$ \\
\hline C & 2.743158 & 2.208738 & -0.177037 \\
\hline C & 2.379233 & -1.405992 & 0.026657 \\
\hline C & $\odot .953280$ & -1.409841 & $\odot .036564$ \\
\hline C & $\odot .265444$ & -2.623091 & -0.151257 \\
\hline C & 0.956613 & -3.800080 & -0.426975 \\
\hline C & 2.352312 & -3.786692 & -0.493771 \\
\hline C & 3.052389 & -2.600517 & -0.267882 \\
\hline $\mathrm{N}$ & -1.811732 & 0.067681 & -1.052287 \\
\hline C & -2.827161 & -0.096136 & -0.165313 \\
\hline C & -4.118045 & -0.495404 & -0.573280 \\
\hline C & -4.338080 & $-\odot .727955$ & -1.932885 \\
\hline C & -3.299993 & -0.546214 & -2.841534 \\
\hline C & -2.063197 & -0.139774 & -2.364617 \\
\hline C & -2.565799 & 0.181430 & 1.281078 \\
\hline C & -2.547489 & 1.502223 & 1.752452 \\
\hline C & -2.353989 & 1.763989 & 3.108751 \\
\hline C & -2.172112 & 0.711686 & 4. . $\odot 94 \odot 8$ \\
\hline C & -2.192135 & -0.605779 & 3.548663 \\
\hline C & -2.397229 & -0.869209 & 2.192651 \\
\hline C & -5.246084 & -0.649771 & $\odot .421157$ \\
\hline H & $-6.1900 \odot 4$ & -0.844232 & -0.097410 \\
\hline $\mathrm{H}$ & -5.368777 & 0.252056 & 1.030273 \\
\hline $\mathrm{H}$ & -5.067300 & -1.477219 & 1.117026 \\
\hline C & 5.235984 & -0.976237 & 1.226478 \\
\hline $\mathrm{H}$ & 4.661016 & -1.744597 & 1.751141 \\
\hline H & 5.960292 & -0.552853 & 1.929854 \\
\hline $\mathrm{H}$ & 5.812037 & -1.477379 & 0.438040 \\
\hline $\mathrm{H}$ & 5.819349 & 1.631308 & 1.098564 \\
\hline $\mathrm{H}$ & 4.389049 & 3.512508 & 0.268870 \\
\hline $\mathrm{H}$ & 2.060552 & 2.974779 & -0.524740 \\
\hline H & 2.898687 & -4.696731 & -0.725033 \\
\hline $\mathrm{H}$ & 0.410816 & -4.724933 & -0.595014 \\
\hline $\mathrm{H}$ & -0.818718 & -2.627806 & $-\odot .096272$ \\
\hline $\mathrm{H}$ & -1.234976 & 0.051175 & -3.036776 \\
\hline H & -3.442644 & -0.702151 & -3.905917 \\
\hline $\mathrm{H}$ & -5.323647 & -1.034524 & -2.274039 \\
\hline $\mathrm{H}$ & -2.051330 & -1.429936 & 4.242951 \\
\hline $\mathrm{H}$ & -2.017215 & $\odot .917756$ & 5.065099 \\
\hline $\mathrm{H}$ & -2.338813 & 2.791920 & 3.460663 \\
\hline $\mathrm{H}$ & 4.130535 & -2.605644 & -0.371204 \\
\hline
\end{tabular}




$\begin{array}{lrrr}\text { H } & -2.416080 & -1.897118 & 1.838822 \\ \text { Cl } & 0.030124 & 2.411681 & -2.148269 \\ \text { H } & -2.670509 & 2.320631 & 1.049525 \\ \text { H } & 0.386857 & -0.621494 & 0.706117 \\ \text { H } & -0.099112 & 1.414444 & 0.629399\end{array}$

10

B3LYP SCF energy:

-2051.57619241 a.u.

B3LYP enthalpy:

B3LYP free energy:

-2051.151526 a.u.

-2051.237801 a.u.

M06 SCF energy in solution:

-2052.07384131 a.u.

M06 enthalpy in solution:

-2051.649175 a.u.

M06 free energy in solution: $\quad-2051.735450$ a.u.

Three lowest frequencies (cm-1): $24.2271 \quad 39.2214 \quad 43.2063$

Cartesian coordinates

\begin{tabular}{|c|c|c|c|}
\hline ATOM & $x$ & Y & Z \\
\hline $\mathrm{Ru}$ & -0.178653 & $-\odot .669796$ & -0.738648 \\
\hline $\mathrm{N}$ & 3.103747 & 1.419542 & -0.687639 \\
\hline C & 3.165298 & $0.4 \odot 4253$ & $\odot .191797$ \\
\hline C & 4.196091 & -0.563590 & 0.163677 \\
\hline C & 5.175731 & -0.418638 & -0.824982 \\
\hline C & 5.119540 & $\odot .640534$ & -1.728389 \\
\hline C & 4.056284 & 1.532253 & -1.621003 \\
\hline C & 2.105472 & ๑. 361461 & 1.257035 \\
\hline C & 0.753337 & $-\odot .0 \odot \odot 325$ & 1.093670 \\
\hline C & -0.076495 & -0.042512 & 2.221305 \\
\hline C & 0.392738 & ๑. 291087 & 3.490769 \\
\hline C & 1.718321 & 0.686934 & 3.657546 \\
\hline C & 2.554213 & $\odot .710744$ & 2.547954 \\
\hline $\mathrm{N}$ & -2.119571 & -1.020025 & -0.099352 \\
\hline C & -2.936381 & 0.061957 & $\odot .109620$ \\
\hline C & -4.317670 & $-\odot .15430 \odot$ & $\odot .364213$ \\
\hline C & $-4.78009 \odot$ & -1.471445 & $\odot .399438$ \\
\hline C & -3.926185 & -2.548227 & $\odot .186933$ \\
\hline C & -2.593359 & -2.278135 & -0.067408 \\
\hline C & -2.217287 & 1.338726 & 0.013162 \\
\hline C & $-\odot .855477$ & 1.261526 & -0.361176 \\
\hline C & $-\odot . \odot 88826$ & 2.412038 & $-\odot .521697$ \\
\hline C & $-\odot .663207$ & 3.665015 & -0.308298 \\
\hline C & -1.998541 & 3.766779 & $\odot .087885$ \\
\hline C & -2.765240 & 2.619504 & $\odot .249046$ \\
\hline C & -5.335823 & $\odot .942273$ & 0.574034 \\
\hline $\mathrm{H}$ & -6.334582 & $\odot .505097$ & $\odot .662751$ \\
\hline
\end{tabular}




\begin{tabular}{|c|c|c|c|}
\hline $\mathrm{H}$ & -5.356860 & 1.647547 & -0.263386 \\
\hline $\mathrm{H}$ & -5.148351 & 1.514745 & 1.489661 \\
\hline C & 4.244230 & -1.725511 & 1.127593 \\
\hline $\mathrm{H}$ & 3.283005 & -2.247996 & 1.163944 \\
\hline $\mathrm{H}$ & 4.472730 & -1.398143 & 2.148385 \\
\hline $\mathrm{H}$ & 5.015247 & -2.441487 & $\odot .825062$ \\
\hline $\mathrm{H}$ & 5.980848 & -1.147317 & -0.886438 \\
\hline $\mathrm{H}$ & 5.872694 & $\odot .76504 \odot$ & -2.500647 \\
\hline H & 3.954906 & 2.369563 & -2.308924 \\
\hline $\mathrm{H}$ & 2.098213 & $\odot .967726$ & 4.635927 \\
\hline $\mathrm{H}$ & $-\odot .283735$ & 0.243486 & 4.340561 \\
\hline $\mathrm{H}$ & -1.111787 & -0.343147 & 2.119285 \\
\hline H & -1.866631 & -3.061278 & $-\odot .238101$ \\
\hline H & -4.279261 & -3.573162 & $\odot .211149$ \\
\hline $\mathrm{H}$ & -5.835363 & -1.648270 & $\odot .589718$ \\
\hline $\mathrm{H}$ & -2.444243 & 4.739476 & $\odot .276024$ \\
\hline $\mathrm{H}$ & -0.060550 & 4.560145 & -0.440145 \\
\hline H & $\odot .962756$ & 2.335966 & $-\odot .781442$ \\
\hline $\mathrm{H}$ & 3.593122 & 1.010019 & 2.664550 \\
\hline H & -3.789400 & 2.736018 & $\odot .566036$ \\
\hline $\mathrm{Cl}$ & $\odot .606313$ & -2.761296 & $\odot .128280$ \\
\hline $\mathrm{Cl}$ & -0.970722 & -0.123692 & -2.898744 \\
\hline $\mathrm{H}$ & 1.466076 & -0.771800 & -1.564470 \\
\hline $\mathrm{H}$ & 1.417594 & $\odot .0 \odot 2540$ & -1.4185 \\
\hline
\end{tabular}

11

B3LYP SCF energy:

-1591.18357624 a.u.

B3LYP enthalpy:

-1590.763561 a.u.

B3LYP free energy:

-1590.853599 a.u.

M06 SCF energy in solution:

-1591.68414432 a.u.

M06 enthalpy in solution:

-1591.264129 a.u.

M06 free energy in solution:

-1591.354167 a.u.

Three lowest frequencies (cm-1): $19.4152 \quad 22.9700 \quad 26.0793$

Cartesian coordinates

\begin{tabular}{lcrr} 
ATOM & X & \multicolumn{1}{c}{ Y } & \multicolumn{1}{l}{ Z } \\
Ru & -0.030988 & -0.599538 & -0.373884 \\
N & -2.158382 & -0.998772 & 0.004473 \\
C & -3.006768 & 0.069388 & -0.115204 \\
C & -4.391772 & -0.066997 & 0.115703 \\
C & -4.870134 & -1.354144 & 0.397554 \\
C & -4.009504 & -2.441485 & 0.448414 \\
C & -2.649765 & -2.221423 & 0.258312 \\
C & -2.277510 & 1.290548 & -0.483816
\end{tabular}




\begin{tabular}{|c|c|c|c|}
\hline C & $-\odot .863578$ & 1.206364 & -0.355876 \\
\hline C & -0.048994 & 2.324220 & -0.600151 \\
\hline C & -0.613054 & 3.475634 & -1.155452 \\
\hline C & -1.991037 & 3.538233 & -1.363452 \\
\hline C & -2.819857 & 2.462077 & -1.026197 \\
\hline $\mathrm{N}$ & 1.911324 & -0.397381 & -1.041571 \\
\hline C & 2.885253 & $\odot .021467$ & -0.172117 \\
\hline C & 4.233576 & $\odot .050762$ & $-\odot .607159$ \\
\hline C & 4.500822 & -0.347999 & -1.921331 \\
\hline C & 3.490140 & -0.765530 & -2.785778 \\
\hline C & 2.195826 & -0.782363 & -2.299633 \\
\hline C & 2.318133 & $\odot .380446$ & 1.131821 \\
\hline C & $\odot .923974$ & $\odot .182481$ & 1.256646 \\
\hline C & ๑. 259101 & $\odot .449668$ & 2.445563 \\
\hline C & $\odot .973275$ & $\odot .945120$ & 3.541894 \\
\hline C & 2.346937 & 1.168471 & 3.437802 \\
\hline C & 3.014720 & $\odot .888431$ & 2.249623 \\
\hline C & 5.396034 & $\odot .469144$ & $\odot .259559$ \\
\hline $\mathrm{H}$ & 6.332655 & 0.357461 & -0.292541 \\
\hline H & 5.470768 & -0.144539 & 1.163687 \\
\hline $\mathrm{H}$ & 5.321418 & 1.517800 & 0.568634 \\
\hline C & -5.375293 & 1.079139 & $\odot .104891$ \\
\hline H & -4.986055 & 1.962405 & $\odot .619845$ \\
\hline H & -6.298607 & 0.779754 & 0.608131 \\
\hline $\mathrm{H}$ & -5.649569 & 1.375616 & -0.915063 \\
\hline $\mathrm{H}$ & -5.931977 & -1.491570 & $\odot .581774$ \\
\hline H & -4.369570 & -3.442377 & $\odot .659422$ \\
\hline $\mathrm{H}$ & -1.927970 & -3.023213 & $\odot .333632$ \\
\hline $\mathrm{H}$ & -2.435503 & 4.437997 & -1.778247 \\
\hline H & ๑. 019531 & 4.324145 & -1.398381 \\
\hline H & 1.012832 & 2.291864 & -0.387655 \\
\hline $\mathrm{H}$ & 1.356079 & -1.107477 & -2.906862 \\
\hline H & 3.700710 & -1.076101 & -3.802691 \\
\hline $\mathrm{H}$ & 5.529703 & -0.334267 & -2.269360 \\
\hline $\mathrm{H}$ & 2.903140 & 1.556988 & 4.285051 \\
\hline H & $\odot .453436$ & 1.152273 & 4.472988 \\
\hline H & $-\odot .807127$ & $\odot .267523$ & 2.532048 \\
\hline $\mathrm{H}$ & -3.880147 & 2.546509 & -1.218585 \\
\hline $\mathrm{H}$ & 4.078231 & 1.068102 & 2.205901 \\
\hline $\mathrm{Cl}$ & $\odot .440696$ & -2.618851 & 0.724093 \\
\hline $\mathrm{H}$ & $-\odot .921224$ & -2.567848 & -3.966876 \\
\hline $\mathrm{H}$ & -1.092834 & -1.907719 & -4.263565 \\
\hline
\end{tabular}


B3LYP SCF energy:

B3LYP enthalpy:

B3LYP free energy:

M06 SCF energy in solution:

M06 enthalpy in solution:

M06 free energy in solution:

Three lowest frequencies (cm-1):
-2051.67585356 a.u.

-2051.244780 a.u.

-2051.333282 a.u.

-2052.15826163 a.u.

-2051.727188 a.u.

-2051.815690 a.u.

$\begin{array}{lll}23.6499 & 29.9165 & 32.1207\end{array}$

Cartesian coordinates

\begin{tabular}{|c|c|c|c|}
\hline ATOM & $x$ & Y & z \\
\hline $\mathrm{Ru}$ & $-\odot .0 \odot \odot 872$ & $-\odot .94 \odot \odot 75$ & $\odot . \odot \odot \odot 165$ \\
\hline $\mathrm{N}$ & -2.080425 & -0.878325 & ๑. 394312 \\
\hline C & -2.775244 & 0.176310 & -0.102779 \\
\hline C & -4.166740 & ๑. 296892 & $\odot .069719$ \\
\hline C & -4.816130 & $-\odot .719789$ & $\odot .780261$ \\
\hline C & -4.096765 & -1.801255 & 1.277454 \\
\hline C & -2.725676 & -1.854281 & 1.056784 \\
\hline C & -1.957248 & 1.156762 & -0.877041 \\
\hline C & -1.181889 & 0.712162 & -1.968489 \\
\hline C & $-\odot .417728$ & 1.624313 & -2.707897 \\
\hline C & -0.433939 & 2.980492 & -2.388117 \\
\hline C & -1.209907 & 3.427052 & -1.313721 \\
\hline C & -1.958385 & 2.525192 & -0.560034 \\
\hline$N$ & 2.079108 & $-\odot .878867$ & -0.393942 \\
\hline C & 2.775252 & $\odot .175037$ & $\odot .102764$ \\
\hline C & 4.167086 & $\odot .293363$ & -0.069038 \\
\hline C & 4.815314 & -0.724569 & -0.778787 \\
\hline C & 4.094525 & -1.805121 & -1.275946 \\
\hline C & 2.723310 & -1.856018 & -1.055782 \\
\hline C & 1.958643 & 1.157388 & $\odot .876077$ \\
\hline C & 1.181381 & 0.714835 & 1.966932 \\
\hline C & $\odot .418450$ & 1.628796 & 2.705349 \\
\hline C & $\odot .437668$ & 2.984818 & 2.385044 \\
\hline C & 1.215376 & 3.429356 & 1.311076 \\
\hline C & 1.962703 & 2.525658 & $\odot .558421$ \\
\hline C & 4.968928 & 1.438492 & $\odot .507251$ \\
\hline $\mathrm{H}$ & 6.014325 & 1.140777 & 0.636965 \\
\hline $\mathrm{H}$ & 4.582400 & 1.759882 & 1.478474 \\
\hline $\mathrm{H}$ & 4.961164 & 2.316222 & -0.151171 \\
\hline C & -4.966945 & 1.443215 & -0.506393 \\
\hline $\mathrm{H}$ & -4.579786 & 1.764303 & -1.477451 \\
\hline $\mathrm{H}$ & -4.957984 & 2.320765 & $\odot .152244$ \\
\hline $\mathrm{H}$ & -6.012737 & 1.147042 & $-\odot .63630 \odot$ \\
\hline $\mathrm{H}$ & -5.892753 & $-\odot .665563$ & $\odot .922292$ \\
\hline $\mathrm{H}$ & -4.586907 & -2.604908 & 1.817336 \\
\hline $\mathrm{H}$ & -2.103137 & -2.668017 & 1.409125 \\
\hline
\end{tabular}




$\begin{array}{lrrr}\text { H } & -1.226044 & 4.482990 & -1.057056 \\ \text { H } & 0.146255 & 3.688951 & -2.973248 \\ \text { H } & 0.166387 & 1.264216 & -3.550258 \\ \text { H } & 2.099819 & -2.669108 & -1.407933 \\ \text { H } & 4.583653 & -2.609736 & -1.815317 \\ \text { H } & 5.892099 & -0.672080 & -0.920231 \\ \text { H } & 1.233827 & 4.485150 & 1.053956 \\ \text { H } & -0.141564 & 3.694702 & 2.969405 \\ \text { H } & -0.167117 & 1.270200 & 3.547347 \\ \text { H } & -2.536388 & 2.879845 & 0.287871 \\ \text { H } & 2.542067 & 2.878815 & -0.289179 \\ \text { Cl } & -.456804 & -2.601087 & 1.662139 \\ \text { H } & 1.227172 & -0.324028 & 2.282159 \\ \text { Cl } & -0.459114 & -2.602718 & -1.660032 \\ \text { H } & -1.230124 & -0.326668 & -2.283357\end{array}$

13

B3LYP SCF energy:

-1131.15244396 a.u.

B3LYP enthalpy:

-1130.735642 a.u.

B3LYP free energy:

-1130.812309 a.u.

M06 SCF energy in solution:

-1131.62706120 a.u.

M06 enthalpy in solution:

-1131.210259 a.u.

M06 free energy in solution:

-1131.286926 a.u.

Three lowest frequencies $(\mathrm{cm}-1): \quad 22.8701 \quad 30.3610 \quad 33.3996$

Cartesian coordinates

\begin{tabular}{|c|c|c|c|}
\hline АTOM & $x$ & Y & Z \\
\hline $\mathrm{Ru}$ & $\odot .008032$ & $-\odot .9 \odot 3179$ & $-\odot .454856$ \\
\hline$N$ & -2.139993 & -0.572544 & -1.057983 \\
\hline C & -3.003865 & -0.067076 & -0.135682 \\
\hline C & -4.352440 & $\odot .205894$ & -0.486707 \\
\hline C & -4.754462 & -0.099731 & -1.788667 \\
\hline C & -3.864309 & $-\odot .652354$ & -2.706550 \\
\hline C & -2.557533 & $-\odot .867602$ & -2.294838 \\
\hline C & -2.336214 & $\odot .112746$ & 1.161956 \\
\hline C & -0.942162 & -0.152717 & 1.146208 \\
\hline C & $-\odot .19829 \odot$ & $-\odot .056333$ & 2.329851 \\
\hline C & -0.826648 & $\odot .255647$ & 3.537539 \\
\hline C & -2.199536 & $\odot .506809$ & 3.563061 \\
\hline C & -2.946051 & $\odot .440149$ & 2.388441 \\
\hline$N$ & 1.095510 & 1.097332 & $-\odot .303956$ \\
\hline C & 2.453653 & 1. 016796 & -0.306449 \\
\hline C & 3.227930 & 2.180604 & -0.564450 \\
\hline C & 2.547464 & 3.390903 & $-\odot .730450$ \\
\hline
\end{tabular}




\begin{tabular}{|c|c|c|c|}
\hline C & 1.159560 & 3.456419 & -0.661675 \\
\hline C & 0.470871 & 2.267294 & -0.464548 \\
\hline C & 2.948596 & $-\odot .359720$ & -0.070174 \\
\hline C & 1.974564 & -1.398513 & -0.172327 \\
\hline C & 2.403177 & -2.724381 & $\odot . \odot 2608 \odot$ \\
\hline C & 3.725371 & -3.028610 & $\odot .362914$ \\
\hline C & 4.652757 & -1.998467 & $\odot .513051$ \\
\hline C & 4.268689 & $-\odot .673923$ & $\odot .297435$ \\
\hline C & 4.731392 & 2.196544 & -0.726326 \\
\hline $\mathrm{H}$ & 5.041320 & 3.125021 & -1.216526 \\
\hline H & 5.086301 & 1.359269 & -1.334828 \\
\hline $\mathrm{H}$ & 5.256986 & 2.150829 & $\odot .235667$ \\
\hline C & -5.369831 & 0.818174 & 0.447127 \\
\hline $\mathrm{H}$ & -5.656286 & $\odot .131861$ & 1.253270 \\
\hline H & -6.280920 & 1.067853 & -0.105334 \\
\hline $\mathrm{H}$ & -5.000367 & 1.738028 & $\odot .913271$ \\
\hline $\mathrm{H}$ & -5.781905 & $\odot .101707$ & -2.081957 \\
\hline H & -4.170987 & -0.900081 & -3.717435 \\
\hline $\mathrm{H}$ & -1.807046 & -1.288504 & -2.954846 \\
\hline H & -2.693732 & 0.756010 & 4.498148 \\
\hline $\mathrm{H}$ & -0.241154 & $\odot .315887$ & 4.451787 \\
\hline H & $\odot .873672$ & -0.225322 & 2.308634 \\
\hline $\mathrm{H}$ & $-\odot .613499$ & 2.233629 & -0.436680 \\
\hline $\mathrm{H}$ & 0.625852 & 4.393645 & -0.781404 \\
\hline $\mathrm{H}$ & 3.123443 & 4.291560 & -0.929607 \\
\hline $\mathrm{H}$ & 5.677557 & -2.218214 & 0.801688 \\
\hline H & 4.026805 & -4.062063 & $\odot .518945$ \\
\hline H & 1.683364 & -3.530763 & -0.073660 \\
\hline $\mathrm{H}$ & -4.009419 & 0.621615 & 2.449669 \\
\hline $\mathrm{H}$ & 5.007884 & 0.099545 & 0.453942 \\
\hline $\mathrm{H}$ & 0.017876 & $-\odot .279169$ & -1.904587 \\
\hline $\mathrm{H}$ & -0.483875 & -2.427320 & -0.789848 \\
\hline
\end{tabular}

14

B3LYP SCF energy:

-1131.18052951 a.u.

B3LYP enthalpy:

-1130.761529 a.u.

B3LYP free energy:

-1130.839893 a.u.

M06 SCF energy in solution:

-1131.65406969 a.u.

M06 enthalpy in solution:

-1131.235069 a.u.

M06 free energy in solution:

-1131.313433 a.u.

Three lowest frequencies (cm-1): $20.6692 \quad 24.2836 \quad 41.0901$

Cartesian coordinates

ATOM $\quad X \quad Y \quad Z$ 


\begin{tabular}{|c|c|c|c|}
\hline $\mathrm{Ru}$ & 0.014714 & -0.109712 & -0.897481 \\
\hline $\mathrm{N}$ & -1.908269 & 0.575136 & -0.928926 \\
\hline C & -2.802836 & $\odot .049368$ & $-\odot .022841$ \\
\hline C & -4.148570 & $\odot .507766$ & -0.037495 \\
\hline C & -4.513872 & 1.473493 & -0.977148 \\
\hline C & -3.588605 & 1.982202 & -1.887914 \\
\hline C & -2.293267 & 1.501474 & -1.824988 \\
\hline C & -2.176477 & -0.966934 & $\odot .827830$ \\
\hline C & $-\odot .805191$ & -1.232461 & $\odot .509196$ \\
\hline C & $-\odot .139196$ & -2.239509 & 1.232989 \\
\hline C & -0.773821 & -2.940236 & 2.254508 \\
\hline C & -2.104242 & -2.655159 & 2.586016 \\
\hline C & -2.796521 & -1.680624 & 1.876430 \\
\hline $\mathrm{N}$ & 1.031130 & 1.477295 & 0.337882 \\
\hline C & 2.304119 & 1.027542 & $\odot .453794$ \\
\hline C & 3.259385 & 1.686124 & 1.252094 \\
\hline C & 2.830267 & 2.831545 & 1.935758 \\
\hline C & 1.517605 & 3.284022 & 1.817897 \\
\hline C & $\odot .644470$ & 2.575657 & 0.998355 \\
\hline C & 2.539441 & -0.193766 & -0.365731 \\
\hline C & 2.142317 & -0.196245 & -1.739565 \\
\hline C & 2.446714 & -1.319946 & -2.550239 \\
\hline C & 3.092426 & -2.420654 & -2.018485 \\
\hline C & 3.480443 & -2.422263 & $-\odot .663941$ \\
\hline C & 3.211271 & -1.330662 & 0.145132 \\
\hline C & 4.694305 & 1.227817 & 1.369903 \\
\hline H & 5.348520 & 2.076306 & 1.596252 \\
\hline $\mathrm{H}$ & 5.047662 & 0.756721 & 0.448505 \\
\hline $\mathrm{H}$ & 4.822136 & $\odot .496169$ & 2.177791 \\
\hline C & -5.222185 & $\odot . \odot \odot 4938$ & ๑. 898887 \\
\hline $\mathrm{H}$ & -5.368166 & -1.077450 & $\odot .811424$ \\
\hline $\mathrm{H}$ & -6.176192 & $\odot .488166$ & $\odot .666099$ \\
\hline $\mathrm{H}$ & -4.990430 & 0.221492 & 1.948188 \\
\hline H & -5.542087 & 1.825719 & $-\odot .994836$ \\
\hline H & -3.863360 & 2.728145 & -2.626223 \\
\hline $\mathrm{H}$ & -1.518774 & 1.846848 & -2.503395 \\
\hline H & -2.599951 & -3.192777 & 3.389963 \\
\hline $\mathrm{H}$ & $-\odot .232939$ & -3.712778 & 2.798401 \\
\hline H & $\odot .890370$ & -2.477486 & $\odot .982216$ \\
\hline $\mathrm{H}$ & $-\odot .392050$ & 2.872448 & $\odot .866256$ \\
\hline $\mathrm{H}$ & 1.178451 & 4.169502 & 2.346293 \\
\hline $\mathrm{H}$ & 3.539356 & 3.375637 & 2.555264 \\
\hline $\mathrm{H}$ & 3.987598 & -3.288317 & -0.247494 \\
\hline $\mathrm{H}$ & 3.303931 & -3.283209 & -2.643901 \\
\hline $\mathrm{H}$ & 2.167595 & -1.298327 & -3.599149 \\
\hline $\mathrm{H}$ & -3.821561 & -1.486215 & 2.156674 \\
\hline
\end{tabular}




$\begin{array}{rrrc}\mathrm{H} & 3.499539 & -1.349503 & 1.191720 \\ \mathrm{H} & 1.886745 & 0.751479 & -2.229896 \\ \mathrm{H} & -0.461701 & -1.303227 & -1.886324\end{array}$

15

B3LYP SCF energy:

-2166.06121505 a.u.

B3LYP enthalpy:

-2165.262506 a.u.

B3LYP free energy:

-2165.385155 a.u.

M06 SCF energy in solution:

-2166.05481944 a.u.

M06 enthalpy in solution:

-2165.256110 a.u.

M06 free energy in solution: $\quad-2165.378759$ a.u.

Three lowest frequencies (cm-1): $17.2919 \quad 25.5622 \quad 26.0025$

Cartesian coordinates

$\begin{array}{lrrr}\text { ATOM } & \text { X } & \text { Y } & \text { Z } \\ \text { Ru } & 0.553176 & 0.218870 & 0.060711 \\ \text { N } & -0.088897 & 1.755318 & 1.294084 \\ \text { C } & 0.487635 & 2.903958 & 0.844246 \\ \text { C } & 0.337308 & 4.126233 & 1.519576 \\ \text { C } & -0.456858 & 4.128948 & 2.677437 \\ \text { C } & -1.058558 & 2.954497 & 3.119255 \\ \text { C } & -0.847727 & 1.780298 & 2.404044 \\ \text { C } & 1.273916 & 2.700306 & -0.411799 \\ \text { C } & 2.285792 & 1.682373 & -0.538168 \\ \text { C } & 3.013555 & 1.666018 & -1.756550 \\ \text { C } & 2.762823 & 2.548268 & -2.794595 \\ \text { C } & 1.745068 & 3.506361 & -2.674120 \\ \text { C } & 1.023142 & 3.575540 & -1.492478 \\ \text { N } & 1.568987 & -1.125273 & -1.241528 \\ \text { C } & 2.912741 & -1.301647 & -0.946925 \\ \text { C } & 3.705864 & -2.136933 & -1.804570 \\ \text { C } & 3.095218 & -2.740521 & -2.894540 \\ \text { C } & 1.735366 & -2.537241 & -3.173927 \\ \text { C } & 1.025122 & -1.710975 & -2.321215 \\ \text { C } & 3.381734 & -0.543267 & 0.191747 \\ \text { C } & 2.604835 & 0.677741 & 0.564438 \\ \text { C } & 2.988536 & 1.264099 & 1.858491 \\ \text { C } & 3.978441 & 0.743892 & 2.648169 \\ \text { C } & 4.700444 & -0.418596 & 2.263492 \\ \text { C } & 4.384432 & -1.025847 & 1.061277 \\ \text { C } & 5.196618 & -2.314659 & -1.629324 \\ \text { H } & 5.649838 & -2.646194 & -2.570020 \\ \text { H } & 5.449092 & -3.065650 & -0.869538 \\ \text { H } & 5.678206 & -1.379848 & -1.322232\end{array}$




\begin{tabular}{|c|c|c|c|}
\hline C & 1.024145 & 5.399183 & 1.079100 \\
\hline $\mathrm{H}$ & 1.979002 & 5.195910 & $\odot .587068$ \\
\hline $\mathrm{H}$ & 1.212224 & 6.045053 & 1.943504 \\
\hline $\mathrm{H}$ & ๑. 411840 & 5.974050 & 0.372644 \\
\hline $\mathrm{H}$ & $-\odot .586085$ & 5.054609 & 3.232966 \\
\hline $\mathrm{H}$ & -1.677842 & 2.939611 & 4.010825 \\
\hline $\mathrm{H}$ & -1.292516 & $\odot .837679$ & 2.700041 \\
\hline $\mathrm{H}$ & 1.530759 & 4.194361 & -3.487661 \\
\hline $\mathrm{H}$ & 3.369187 & 2.505746 & -3.696514 \\
\hline $\mathrm{H}$ & 3.817430 & $\odot .943647$ & -1.851187 \\
\hline $\mathrm{H}$ & -0.029743 & -1.514067 & -2.47493 \\
\hline $\mathrm{H}$ & 1.250641 & -2.997356 & -4.028658 \\
\hline $\mathrm{H}$ & 3.698093 & -3.355134 & -3.56037 \\
\hline $\mathrm{H}$ & 5.464159 & -0.837686 & 2.911801 \\
\hline $\mathrm{H}$ & 4.214474 & 1.232478 & 3.593154 \\
\hline $\mathrm{H}$ & 2.484553 & 2.169052 & 2.184225 \\
\hline $\mathrm{H}$ & $\odot .226072$ & 4.306933 & -1.392409 \\
\hline $\mathrm{H}$ & 4.879899 & -1.959168 & ๑. 816118 \\
\hline $\mathrm{N}$ & 0.145237 & -1.323179 & 1.50205 \\
\hline C & -0.620725 & -2.441129 & 1.35354 \\
\hline C & $-\odot .722507$ & -3.423039 & 2.36752 \\
\hline C & -0.027211 & -3.222143 & 3.56070 \\
\hline C & 0.748422 & -2.077405 & 3.713894 \\
\hline C & 0.811264 & -1.166576 & 2.672226 \\
\hline C & -1.364519 & -2.670968 & $\odot .072708$ \\
\hline C & -2.531849 & -1.960763 & -0.2862 \\
\hline C & -3.207707 & -2.323720 & -1.46246 \\
\hline C & -2.758324 & -3.363628 & -2.27375 \\
\hline C & -1.605753 & -4.063966 & -1.91861 \\
\hline C & $-\odot .922568$ & -3.714658 & $-\odot .75583$ \\
\hline $\mathrm{N}$ & -1.607619 & 0.654624 & -1.586737 \\
\hline C & -2.914670 & 0.835035 & -1.30306 \\
\hline C & -3.821252 & 1.393905 & -2.234586 \\
\hline C & -3.305194 & 1.789138 & -3.473877 \\
\hline C & -1.952846 & 1.629423 & -3.754753 \\
\hline C & -1.144945 & 1.049565 & -2.779930 \\
\hline C & -3.357876 & 0.417551 & ๑. . 06395 \\
\hline C & -3.120202 & -0.883384 & $\odot .565308$ \\
\hline C & -3.539103 & -1.189676 & 1.86939 \\
\hline C & -4.179347 & -0.246110 & 2.67258 \\
\hline C & -4.411550 & 1.036537 & 2.17736 \\
\hline C & -3.999326 & 1.357280 & ๑. 88518 \\
\hline C & -5.304230 & 1.542068 & -1.97040 \\
\hline $\mathrm{H}$ & -5.861288 & 1.514724 & -2.91317 \\
\hline $\mathrm{H}$ & -5.542528 & 2.496211 & -1.483578 \\
\hline $\mathrm{H}$ & -5.687783 & 0.749397 & -1.3221 \\
\hline
\end{tabular}




$\begin{array}{llll}\text { C } & -1.574376 & -4.664053 & 2.204157 \\ \text { H } & -2.556513 & -4.438704 & 1.776099 \\ \text { H } & -1.726599 & -5.148868 & 3.173971 \\ \text { H } & -1.105201 & -5.397030 & 1.537800 \\ \text { H } & -0.096546 & -3.958508 & 4.357460 \\ \text { H } & 1.312482 & -1.887864 & 4.621649 \\ \text { H } & 1.436849 & -0.284707 & 2.745280 \\ \text { H } & -1.234607 & -4.872047 & -2.542778 \\ \text { H } & -3.310145 & -3.627448 & -3.172052 \\ \text { H } & -4.113697 & -1.787870 & -1.730993 \\ \text { H } & -0.084100 & 0.896402 & -2.952890 \\ \text { H } & -1.531789 & 1.931541 & -4.708822 \\ \text { H } & -3.974897 & 2.212586 & -4.219351 \\ \text { H } & -4.903527 & 1.785599 & 2.791881 \\ \text { H } & -4.502310 & -0.517614 & 3.674131 \\ \text { H } & -3.381435 & -2.193863 & 2.249427 \\ \text { H } & -0.014095 & -4.244995 & -0.485731 \\ \text { H } & -4.154467 & 2.364426 & 0.509599\end{array}$

TS1

B3LYP SCF energy:

-2051.61702523 a.u.

B3LYP enthalpy:

-2051.195991 a.u.

B3LYP free energy:

-2051.280480 a.u.

M06 SCF energy in solution:

-2052.11256235 a.u.

M06 enthalpy in solution:

-2051.691528 a.u.

M06 free energy in solution:

-2051.776017 a.u.

Three lowest frequencies (cm-1): $\begin{array}{llll}-152.0002 & 40.0088 & 47.5923\end{array}$

Imaginary frequency:

$-152.0002 \mathrm{~cm}-1$

Cartesian coordinates

\begin{tabular}{|c|c|c|c|}
\hline ATOM & $x$ & Y & Z \\
\hline $\mathrm{Ru}$ & $-\odot .069913$ & $-\odot .268455$ & 1.142281 \\
\hline$N$ & 2.009308 & $-\odot .465822$ & $\odot .844216$ \\
\hline C & 2.520522 & 0.236343 & -0.203442 \\
\hline C & 3.864211 & $\odot .079450$ & -0.597369 \\
\hline C & 4.665092 & -0.789594 & $\odot .154970$ \\
\hline C & 4.136831 & -1.473684 & 1.243220 \\
\hline C & 2.792742 & -1.296198 & 1.550394 \\
\hline C & 1.595602 & 1.214025 & $-\odot .870572$ \\
\hline C & ๑. 302869 & $\odot .875644$ & -1.366620 \\
\hline C & -0.507879 & 1.900415 & -1.883052 \\
\hline C & $-\odot .081289$ & 3.225278 & -1.912109 \\
\hline C & 1.182312 & 3.555025 & -1.428049 \\
\hline C & 2.002136 & 2.554688 & -0.911854 \\
\hline
\end{tabular}




\begin{tabular}{|c|c|c|c|}
\hline$N$ & -2.119935 & $\odot .084003$ & $\odot .923494$ \\
\hline C & -2.493731 & -0.208655 & -0.346591 \\
\hline C & -3.765293 & 0.120268 & -0.839699 \\
\hline C & -4.646511 & $\odot .762265$ & $\odot .041922$ \\
\hline C & -4.257767 & 1.045670 & 1.347157 \\
\hline C & -2.974771 & $\odot .695868$ & 1.755835 \\
\hline C & -1.473482 & -0.998196 & -1.122341 \\
\hline C & $-\odot .16880 \odot$ & $-\odot .544396$ & -1.481842 \\
\hline C & $\odot .695512$ & -1.464239 & -2.119371 \\
\hline C & 0.317941 & -2.770099 & -2.390119 \\
\hline C & -0.961926 & -3.206155 & -2.037165 \\
\hline C & -1.835491 & -2.326993 & -1.414727 \\
\hline C & -4.188286 & -0.193321 & -2.256176 \\
\hline $\mathrm{H}$ & -4.969611 & $\odot .499108$ & -2.585775 \\
\hline $\mathrm{H}$ & -4.595340 & -1.208820 & -2.339770 \\
\hline $\mathrm{H}$ & -3.351689 & -0.126865 & -2.957958 \\
\hline C & 4.457993 & $\odot .781952$ & -1.798615 \\
\hline $\mathrm{H}$ & 3.713712 & $\odot .96480 \odot$ & -2.578331 \\
\hline $\mathrm{H}$ & 5.265635 & $\odot .179148$ & -2.226940 \\
\hline $\mathrm{H}$ & 4.887886 & 1.755077 & -1.531049 \\
\hline $\mathrm{H}$ & 5.705458 & $-\odot .930847$ & -0.127823 \\
\hline $\mathrm{H}$ & 4.743280 & -2.150254 & 1.836285 \\
\hline $\mathrm{H}$ & 2.300377 & -1.832839 & 2.352273 \\
\hline $\mathrm{H}$ & 1.521923 & 4.586340 & -1.427865 \\
\hline $\mathrm{H}$ & -0.734738 & 3.994035 & -2.314749 \\
\hline $\mathrm{H}$ & -1.482282 & 1.642999 & -2.285982 \\
\hline $\mathrm{H}$ & -2.599149 & $\odot .916827$ & 2.747307 \\
\hline $\mathrm{H}$ & -4.928483 & 1.543192 & 2.039702 \\
\hline $\mathrm{H}$ & -5.638076 & 1.041283 & $-\odot .305746$ \\
\hline $\mathrm{H}$ & -1.270411 & -4.228549 & -2.234383 \\
\hline $\mathrm{H}$ & 1.013185 & -3.444036 & -2.882425 \\
\hline $\mathrm{H}$ & 1.675938 & -1.118554 & -2.430235 \\
\hline $\mathrm{H}$ & 2.963279 & 2.823062 & -0.485436 \\
\hline $\mathrm{H}$ & -2.814172 & -2.676973 & -1.101869 \\
\hline $\mathrm{Cl}$ & $-\odot .297234$ & -2.562897 & 1.762599 \\
\hline $\mathrm{Cl}$ & $0.01694 \odot$ & 2.604178 & 2.135765 \\
\hline $\mathrm{H}$ & $\odot .076291$ & $-\odot .029503$ & 2.764767 \\
\hline H & ๑. . 061315 & $\odot .932187$ & 2.486782 \\
\hline
\end{tabular}

TS2a

B3LYP SCF energy:

-2050.43748187 a.u.

B3LYP enthalpy:

-2050.031317 a.u.

B3LYP free energy:

-2050.110839 a.u.

M06 SCF energy in solution:

-2050.93252678 a.u. 
M06 enthalpy in solution:

M06 free energy in solution:

Three lowest frequencies (cm-1):

Imaginary frequency:
-2050.526362 a.u.

-2050.605884 a.u.

$\begin{array}{lll}-241.1629 & 47.4511 & 48.6815\end{array}$

$-241.1629 \mathrm{~cm}-1$

Cartesian coordinates

\begin{tabular}{|c|c|c|c|}
\hline АTOM & $x$ & Y & z \\
\hline $\mathrm{Ru}$ & -0.033812 & -0.927339 & -0.119221 \\
\hline$N$ & -2.105479 & -0.813029 & $\odot .194338$ \\
\hline C & -2.709066 & $\odot .319455$ & $-\odot .261360$ \\
\hline C & -4.055824 & $\odot .597291$ & 0.059142 \\
\hline C & -4.753486 & -0.374100 & $\odot .786906$ \\
\hline C & -4.138501 & -1.558651 & 1.177827 \\
\hline C & -2.793570 & -1.738808 & $\odot .875266$ \\
\hline C & -1.799646 & 1.130936 & -1.093310 \\
\hline C & $-\odot .385853$ & $\odot .936608$ & -0.913270 \\
\hline C & $\odot .486831$ & 1.523222 & -1.859076 \\
\hline C & $\odot .002862$ & 2.273489 & -2.926715 \\
\hline C & -1.368695 & 2.478900 & -3.080672 \\
\hline C & -2.254396 & 1.895471 & -2.175915 \\
\hline$N$ & 2.069527 & $-\odot .855917$ & $-\odot .361850$ \\
\hline C & 2.703259 & $\odot .246151$ & 0.140206 \\
\hline C & 4.068873 & 0.473706 & -0.159044 \\
\hline C & $4.74950 \odot$ & -0.514116 & -0.880729 \\
\hline C & 4.104443 & -1.670245 & -1.300907 \\
\hline C & 2.745968 & -1.794933 & -1.037931 \\
\hline C & 1.826870 & 1.116335 & $\odot .955056$ \\
\hline C & $\odot .413469$ & 0.982530 & 0.810469 \\
\hline C & $-\odot .420852$ & 1.701009 & 1.679487 \\
\hline C & $\odot .097915$ & 2.535933 & 2.664910 \\
\hline C & 1.478783 & 2.655684 & 2.816520 \\
\hline C & 2.328433 & 1.940475 & 1.974330 \\
\hline C & 4.826777 & 1.733482 & 0.198459 \\
\hline $\mathrm{H}$ & 5.662764 & 1.868602 & -0.495347 \\
\hline $\mathrm{H}$ & 5.254169 & 1.690504 & 1.208142 \\
\hline $\mathrm{H}$ & 4.194746 & 2.624332 & 0.146719 \\
\hline C & -4.759590 & 1.886816 & $-\odot .298926$ \\
\hline $\mathrm{H}$ & -4.079988 & 2.743715 & -0.283786 \\
\hline $\mathrm{H}$ & -5.566550 & 2.080372 & 0.415063 \\
\hline $\mathrm{H}$ & -5.217793 & 1.846783 & -1.295601 \\
\hline $\mathrm{H}$ & -5.790891 & -0.186344 & 1.053220 \\
\hline $\mathrm{H}$ & -4.676091 & -2.319710 & 1.733107 \\
\hline $\mathrm{H}$ & -2.233521 & -2.612751 & 1.180541 \\
\hline $\mathrm{H}$ & -1.750529 & 3.060586 & -3.914338 \\
\hline $\mathrm{H}$ & $\odot .7 \odot 4574$ & 2.701782 & -3.637610 \\
\hline $\mathrm{H}$ & 1.557239 & 1.402982 & -1.750072 \\
\hline
\end{tabular}




$\begin{array}{lrrc}\text { H } & 2.159932 & -2.645153 & -1.368483 \\ \text { H } & 4.626225 & -2.447345 & -1.849156 \\ \text { H } & 5.798799 & -0.358436 & -1.119603 \\ \text { H } & 1.897068 & 3.281929 & 3.599177 \\ H & -0.576366 & 3.076303 & 3.324207 \\ H & -1.496956 & 1.605495 & 1.588651 \\ H & -3.318173 & 1.981839 & -2.360056 \\ \text { H } & 3.392408 & 1.982080 & 2.164695 \\ \text { Cl } & -0.295850 & -3.144608 & -1.006061 \\ \text { Cl } & 0.227102 & -1.652563 & 2.133973\end{array}$

TS2b

$\begin{array}{lll}\text { B3LYP SCF energy: } & -1590.81551080 \mathrm{a} \cdot \mathrm{u} . \\ \text { B3LYP enthalpy: } & -1590.404352 \mathrm{a} \cdot \mathrm{u} . \\ \text { B3LYP free energy: } & -1590.479964 \mathrm{a} \cdot \mathrm{u} . \\ \text { M06 SCF energy in solution: } & -1591.29959353 \mathrm{a} \cdot \mathrm{u} . & \\ \text { M06 enthalpy in solution: } & -1590.888435 \mathrm{a} \cdot \mathrm{u} . \\ \text { M06 free energy in solution: } & -1590.964047 \mathrm{a} \cdot \mathrm{u} . & \\ \text { Three lowest frequencies (cm-1): } & -154.6410 \quad 50.2347 & 55.2471 \\ \text { Imaginary frequency: } & -154.6410 \mathrm{~cm}-1 & \end{array}$

Cartesian coordinates

\begin{tabular}{|c|c|c|c|}
\hline ATOM & $x$ & Y & Z \\
\hline $\mathrm{Ru}$ & -0.012393 & 1.064212 & $-\odot .097993$ \\
\hline$N$ & -2.093371 & $\odot .912707$ & -0.323479 \\
\hline C & -2.667686 & -0.192125 & $\odot .235200$ \\
\hline C & $-4.01360 \odot$ & $-\odot .525415$ & $-\odot .040832$ \\
\hline C & -4.745856 & $\odot .359897$ & $-\odot .839390$ \\
\hline C & -4.163140 & 1.520300 & -1.340834 \\
\hline C & -2.821003 & 1.756885 & -1.071294 \\
\hline C & -1.735278 & $-\odot .930697$ & 1.110369 \\
\hline C & -0.319164 & -0.806333 & ๑.853948 \\
\hline C & ๑.552848 & -1.301820 & 1.857928 \\
\hline C & ๑. 082750 & -1.909359 & 3.015895 \\
\hline C & -1.289075 & -2.054058 & 3.232349 \\
\hline C & -2.179252 & -1.549107 & 2.286422 \\
\hline$N$ & 2.084946 & $\odot .981535$ & $\odot .092625$ \\
\hline C & 2.674220 & $-\odot .198950$ & $-\odot .265929$ \\
\hline C & 4. . 030001 & -0.450234 & $\odot .051521$ \\
\hline C & 4.756964 & $\odot .582038$ & 0.656160 \\
\hline C & 4.159689 & 1.804964 & $\odot .940940$ \\
\hline C & 2.808253 & 1.960882 & $\odot .658694$ \\
\hline C & 1.757026 & -1.122974 & -0.966588 \\
\hline C & ๑.340842 & $-\odot .989604$ & -0.748659 \\
\hline
\end{tabular}




$\begin{array}{lrrr}\text { C } & -0.510626 & -1.753888 & -1.579910 \\ \text { C } & -0.022019 & -2.609286 & -2.558912 \\ \text { C } & 1.354234 & -2.731894 & -2.759791 \\ \text { C } & 2.224256 & -1.979847 & -1.972640 \\ \text { C } & 4.719728 & -1.779214 & -0.165754 \\ \text { H } & 5.526505 & -1.901008 & 0.564551 \\ \text { H } & 5.176890 & -1.853734 & -1.160695 \\ \text { H } & 4.031466 & -2.622051 & -0.058217 \\ \text { C } & -4.678060 & -1.797949 & 0.435067 \\ \text { H } & -3.969270 & -2.627434 & 0.509288 \\ \text { H } & -5.472026 & -2.087183 & -0.261405 \\ \text { H } & -5.146276 & -1.677829 & 1.420506 \\ \text { H } & -5.780887 & 0.124206 & -1.075235 \\ \text { H } & -4.725609 & 2.217781 & -1.952602 \\ \text { H } & -2.290448 & 2.625247 & -1.442889 \\ \text { H } & -1.663201 & -2.520742 & 4.138610 \\ \text { H } & 0.794677 & -2.271729 & 3.753234 \\ \text { H } & 1.623752 & -1.231629 & 1.711493 \\ \text { H } & 2.263653 & 2.872822 & 0.876145 \\ \text { H } & 4.715786 & 2.615404 & 1.400471 \\ \text { H } & 5.799002 & 0.410141 & 0.915013 \\ \text { H } & 1.747329 & -3.383615 & -3.534463 \\ \text { H } & -0.718524 & -3.173777 & -3.173647 \\ \text { H } & -1.583200 & -1.682146 & -1.442937 \\ \text { H } & -3.240169 & -1.573141 & 2.506151 \\ \text { H } & 3.283522 & -2.013969 & -2.196012 \\ \text { Cl } & -0.101798 & 3.454961 & -0.003271 \\ \text { H } & 0.065550 & 1.121757 & -1.657861\end{array}$

TS2b-1

\begin{tabular}{|c|c|c|}
\hline B3LYP SCF energy: & -1320.41115332 a.u. & \\
\hline B3LYP enthalpy: & -1320.116090 a.u. & \\
\hline B3LYP free energy: & -1320.181724 a.u. & \\
\hline M06 SCF energy in solution: & -1321.03320816 a.u. & \\
\hline M06 enthalpy in solution: & -1320.738145 a.u. & \\
\hline M06 free energy in solution: & -1320.803779 a.u. & \\
\hline Three lowest frequencies $(\mathrm{cm}-1)$ : & -212.1129 & 59.5376 \\
\hline Imaginary frequency: & $-212.1129 \mathrm{~cm}-1$ & \\
\hline
\end{tabular}

Cartesian coordinates

$\begin{array}{lccc}\text { ATOM } & \text { X } & \text { Y } & \text { Z } \\ \text { Ru } & -0.750718 & 0.941001 & -0.650677 \\ \text { C } & -1.400980 & -0.584556 & 0.563262 \\ \text { C } & -1.009110 & -0.798549 & 1.905772\end{array}$




\begin{tabular}{|c|c|c|c|}
\hline C & -1.997559 & -1.042374 & 2.853377 \\
\hline C & -3.340044 & -1.083522 & 2.463928 \\
\hline C & -3.636577 & -0.858900 & 1.117376 \\
\hline $\mathrm{N}$ & 1.164674 & 1.045733 & -0.001436 \\
\hline C & 1.846957 & -0.135814 & $\odot .150378$ \\
\hline C & 3.082012 & -0.153212 & $\odot .837891$ \\
\hline C & 3.609367 & 1.069526 & 1.269433 \\
\hline C & 2.934423 & 2.262377 & 1.035551 \\
\hline C & 1.698459 & 2.212889 & 0.406511 \\
\hline C & 1.148761 & -1.288136 & -0.451645 \\
\hline C & $-\odot .255904$ & -1.159087 & -0.692819 \\
\hline C & - ๑. 899085 & -2.158766 & -1.449217 \\
\hline C & $-\odot .202970$ & -3.254290 & -1.946933 \\
\hline C & 1.167160 & -3.380227 & -1.703959 \\
\hline C & 1.833056 & -2.396366 & -0.974694 \\
\hline C & 3.838812 & -1.418254 & 1.176234 \\
\hline $\mathrm{H}$ & 4.468652 & -1.248014 & 2.055104 \\
\hline $\mathrm{H}$ & 4.504061 & -1.733065 & 0.362364 \\
\hline $\mathrm{H}$ & 3.167906 & -2.253652 & 1.394593 \\
\hline H & -4.133872 & -1.271746 & 3.179051 \\
\hline $\mathrm{H}$ & -1.721924 & -1.197715 & 3.893523 \\
\hline H & $\odot .038440$ & $-\odot .77470 \odot$ & 2.185218 \\
\hline H & 1.096666 & 3.092493 & $\odot .212742$ \\
\hline $\mathrm{H}$ & 3.338019 & 3.216916 & 1.355738 \\
\hline $\mathrm{H}$ & 4.555888 & 1.074870 & 1.803750 \\
\hline $\mathrm{H}$ & 1.721747 & -4.226869 & -2.097967 \\
\hline H & $-\odot .726927$ & $-4.0 \odot 6572$ & -2.530524 \\
\hline $\mathrm{H}$ & -1.966768 & -2.064897 & -1.617663 \\
\hline $\mathrm{H}$ & -4.666485 & -0.853399 & 0.765559 \\
\hline $\mathrm{H}$ & 2.908350 & -2.468269 & -0.873673 \\
\hline $\mathrm{Cl}$ & -1.115640 & 3.221619 & -1.179650 \\
\hline H & $-\odot .333127$ & 0.613356 & -2.118668 \\
\hline $\mathrm{N}$ & -2.700934 & -0.626696 & $\odot .194580$ \\
\hline
\end{tabular}

TS2C

B3LYP SCF energy:

-1131.14718258 a.u.

B3LYP enthalpy:

B3LYP free energy:

-1130.732277 a.u.

-1130.804935 a.u.

M06 SCF energy in solution:

-1131.62498571 a.u.

M06 enthalpy in solution:

-1131.210080 a.u.

M06 free energy in solution: $\quad-1131.282738 \mathrm{a} . \mathrm{u}$.

Three lowest frequencies (cm-1): $\begin{array}{llll}-239.9540 & 45.1847 & 50.4130\end{array}$

Imaginary frequency: $\quad-239.9540 \mathrm{~cm}-1$ 
Cartesian coordinates

\begin{tabular}{|c|c|c|c|}
\hline ATOM & $x$ & Y & Z \\
\hline $\mathrm{Ru}$ & 0.001816 & -1.303289 & -0.032352 \\
\hline$N$ & 2.094837 & -1.207333 & -0.238751 \\
\hline C & 2.698888 & -0.078338 & $\odot .241335$ \\
\hline C & 4.045325 & $\odot .215069$ & -0.076723 \\
\hline C & 4.756098 & -0.730702 & -0.822935 \\
\hline C & 4.148986 & -1.912045 & -1.239671 \\
\hline C & 2.807658 & -2.106304 & $-\odot .938926$ \\
\hline C & 1.793085 & $\odot .734203$ & 1.079158 \\
\hline C & ๑. 381328 & $\odot .593764$ & $\odot .862227$ \\
\hline C & -0.478927 & 1.190893 & 1.807941 \\
\hline C & $\odot .005747$ & 1.908089 & 2.899702 \\
\hline C & 1.380413 & 2.059805 & 3.085116 \\
\hline C & 2.260097 & 1.461502 & 2.183810 \\
\hline $\mathrm{N}$ & -2.079527 & -1.225280 & 0.137365 \\
\hline C & -2.688753 & -0.063873 & -0.262967 \\
\hline C & -4.044641 & 0.180362 & $\odot .073921$ \\
\hline C & -4.746036 & $-\odot .829487$ & $\odot .739057$ \\
\hline C & -4.128857 & -2.032768 & 1.071274 \\
\hline C & -2.785689 & -2.184816 & 0.760970 \\
\hline C & -1.790286 & 0.831651 & -1.013050 \\
\hline C & $-\odot .374465$ & 0.644541 & -0.854930 \\
\hline C & 0.472004 & 1.383454 & -1.711566 \\
\hline C & -0.024747 & 2.261243 & -2.666698 \\
\hline C & -1.403998 & 2.436009 & -2.814646 \\
\hline C & -2.268460 & 1.713892 & -1.997979 \\
\hline C & -4.757760 & 1.489332 & -0.184128 \\
\hline $\mathrm{H}$ & -5.577529 & 1.611843 & 0.531453 \\
\hline $\mathrm{H}$ & $-5.2 \odot \odot 172$ & 1.533536 & -1.187447 \\
\hline $\mathrm{H}$ & -4.086532 & 2.347131 & -0.085409 \\
\hline C & 4.734333 & 1.508802 & $\odot .297073$ \\
\hline $\mathrm{H}$ & 4.040151 & 2.353656 & 0.307073 \\
\hline $\mathrm{H}$ & 5.529854 & 1.727814 & -0.422783 \\
\hline $\mathrm{H}$ & 5.204466 & 1.458641 & 1.287639 \\
\hline $\mathrm{H}$ & 5.791005 & $-\odot .526183$ & -1.086604 \\
\hline $\mathrm{H}$ & 4.691554 & -2.656671 & -1.812865 \\
\hline $\mathrm{H}$ & 2.258555 & -2.980839 & -1.264243 \\
\hline $\mathrm{H}$ & 1.767656 & 2.611517 & 3.936938 \\
\hline $\mathrm{H}$ & -0.693798 & 2.350307 & 3.605038 \\
\hline $\mathrm{H}$ & -1.552318 & 1.112923 & 1.673525 \\
\hline $\mathrm{H}$ & -2.230152 & -3.082561 & 1.005793 \\
\hline $\mathrm{H}$ & -4.666543 & -2.826991 & 1.578567 \\
\hline $\mathrm{H}$ & -5.785256 & $-\odot .657898$ & 1.009109 \\
\hline $\mathrm{H}$ & -1.801710 & 3.105581 & -3.571769 \\
\hline $\mathrm{H}$ & 0.666561 & 2.805670 & -3.305943 \\
\hline
\end{tabular}




$\begin{array}{rrrr}\mathrm{H} & 1.546638 & 1.267730 & -1.620424 \\ \mathrm{H} & 3.323412 & 1.506387 & 2.388490 \\ \mathrm{H} & -3.333103 & 1.791679 & -2.179812 \\ \mathrm{H} & -0.008023 & -1.573936 & -1.581353 \\ \mathrm{H} & -0.019852 & -2.951986 & -0.175968\end{array}$

TS3

B3LYP SCF energy:

-1590.83017796 a.u.

B3LYP enthalpy:

-1590.418843 a.u.

B3LYP free energy:

-1590.497161 a.u.

M06 SCF energy in solution:

-1591.31556556 a.u.

M06 enthalpy in solution:

-1590.904231 a.u.

M06 free energy in solution: -1590.982549 a.u.

Three lowest frequencies (cm-1): $\begin{array}{llll}-157.9184 & 29.2579 & 29.5690\end{array}$

Imaginary frequency:

$-157.9184 \mathrm{~cm}-1$

Cartesian coordinates

\begin{tabular}{lccc} 
ATOM & X & Y & \multicolumn{1}{l}{ Z } \\
Ru & -0.021720 & -0.831352 & -0.218320 \\
N & 2.019462 & -0.748135 & -0.750296 \\
C & 2.841071 & 0.068110 & -0.027129 \\
C & 4.200859 & 0.212525 & -0.405341 \\
C & 4.658338 & -0.540900 & -1.489227 \\
C & 3.812872 & -1.407553 & -2.175949 \\
C & 2.488971 & -1.477401 & -1.772685 \\
C & 2.127280 & 0.702659 & 1.092367 \\
C & 0.724708 & 0.487452 & 1.108214 \\
C & -0.048028 & 1.008374 & 2.150401 \\
C & 0.553181 & 1.706951 & 3.199093 \\
C & 1.934630 & 1.906395 & 3.201783 \\
C & 2.714062 & 1.409986 & 2.160069 \\
N & -2.103829 & -0.942783 & 0.216812 \\
C & -2.909869 & 0.085017 & -0.204399 \\
C & -4.320259 & -0.041857 & -0.092962 \\
C & -4.828431 & -1.188275 & 0.524535 \\
C & -3.987186 & -2.186656 & 1.001293 \\
C & -2.623666 & -2.034008 & 0.807344 \\
C & -2.145917 & 1.221693 & -0.750654 \\
C & -0.746893 & 1.021108 & -0.898362 \\
C & 0.033038 & 2.062324 & -1.422000 \\
C & -0.525260 & 3.292592 & -1.760991 \\
C & -1.891947 & 3.507544 & -1.572680 \\
C & -2.689921 & 2.483049 & -1.073871 \\
C & -5.323856 & 0.956679 & -0.624692
\end{tabular}




$\begin{array}{lrrr}\text { H } & -6.323981 & 0.512654 & -0.615230 \\ \text { H } & -5.101866 & 1.255182 & -1.654030 \\ \text { H } & -5.371074 & 1.868524 & -0.016895 \\ \text { C } & 5.178704 & 1.143451 & 0.272400 \\ \text { H } & 4.769552 & 2.151541 & 0.395823 \\ \text { H } & 6.091017 & 1.223770 & -0.326461 \\ \text { H } & 5.473441 & 0.781309 & 1.264790 \\ \text { H } & 5.696771 & -0.441261 & -1.794993 \\ \text { H } & 4.164481 & -2.006210 & -3.009204 \\ \text { H } & 1.770304 & -2.126550 & -2.258139 \\ \text { H } & 2.409838 & 2.444381 & 4.017354 \\ \text { H } & -0.057727 & 2.097874 & 4.009199 \\ \text { H } & -1.124124 & 0.868081 & 2.147248 \\ \text { H } & -1.906226 & -2.793017 & 1.096869 \\ \text { H } & -4.373203 & -3.077585 & 1.484798 \\ \text { H } & -5.906168 & -1.294927 & 0.620195 \\ \text { H } & -2.338405 & 4.468511 & -1.812687 \\ \text { H } & 0.104765 & 4.085229 & -2.157835 \\ \text { H } & 1.100462 & 1.909379 & -1.554384 \\ \text { H } & 3.783417 & 1.555211 & 2.210066 \\ \text { H } & -3.737146 & 2.692860 & -0.918536 \\ \text { Cl } & 0.424165 & -3.163374 & 0.379718 \\ \text { H } & -0.147587 & -0.377225 & -1.722003\end{array}$

TS4

B3LYP SCF energy:

-1592.02769952 a.u.

B3LYP enthalpy:

-1591.596688 a.u.

B3LYP free energy:

-1591.679980 a.u.

M06 SCF energy in solution:

-1592.51046998 a.u.

M06 enthalpy in solution:

-1592.079458 a.u.

M06 free energy in solution: $\quad-1592.162750$ a.u.

Three lowest frequencies $(\mathrm{cm}-1): \begin{array}{lll}-12.2173 & 23.9509 & 27.6769\end{array}$

Imaginary frequency:

$-12.2173 \mathrm{~cm}-1$

Cartesian coordinates

$\begin{array}{lccc}\text { ATOM } & X & Y & Z \\ \text { Ru } & -0.241482 & -0.225929 & -0.723018 \\ \text { N } & -2.255948 & -0.804912 & -0.542853 \\ \text { C } & -3.085887 & 0.000701 & 0.186935 \\ \text { C } & -4.397550 & -0.433330 & 0.499569 \\ \text { C } & -4.818167 & -1.662065 & -0.018860 \\ \text { C } & -3.976469 & -2.434656 & -0.811640 \\ \text { C } & -2.688046 & -1.974029 & -1.040854 \\ \text { C } & -2.434619 & 1.266768 & 0.576066\end{array}$




\begin{tabular}{|c|c|c|c|}
\hline C & -1.027016 & 1.316740 & 0.375477 \\
\hline C & -0.335680 & 2.494923 & ๑. 691964 \\
\hline C & -1.011982 & 3.627155 & 1.149879 \\
\hline C & -2.397664 & 3.591178 & 1.310080 \\
\hline C & -3.100905 & 2.421862 & 1.025126 \\
\hline$N$ & 1.801107 & $\odot .443945$ & -0.928998 \\
\hline C & 2.846386 & $\odot .200667$ & -0.096982 \\
\hline C & 4.123417 & 0.753865 & -0.329435 \\
\hline C & 4.292857 & 1.572006 & -1.449851 \\
\hline C & 3.223996 & 1.803962 & -2.308626 \\
\hline C & 2.002477 & 1.214194 & -2.019292 \\
\hline C & 2.625449 & -0.707310 & 1.070127 \\
\hline C & 2.670434 & -2.096555 & 0.893200 \\
\hline C & 2.495399 & -2.950766 & 1.982943 \\
\hline C & 2.275614 & -2.426527 & 3.258420 \\
\hline C & 2.233384 & -1.042825 & 3.442017 \\
\hline C & 2.412580 & $-\odot .186989$ & 2.354073 \\
\hline C & 5.302045 & 0.447307 & $\odot .566261$ \\
\hline $\mathrm{H}$ & 6.064170 & 1.228348 & $\odot .479019$ \\
\hline $\mathrm{H}$ & 5.770071 & -0.504410 & 0.282824 \\
\hline $\mathrm{H}$ & 5.013932 & $\odot .360657$ & 1.616054 \\
\hline C & -5.355720 & $\odot .325155$ & 1.389194 \\
\hline $\mathrm{H}$ & -4.862323 & $\odot .714974$ & 2.284922 \\
\hline $\mathrm{H}$ & -6.164874 & $-\odot .337160$ & 1.712466 \\
\hline $\mathrm{H}$ & -5.822073 & 1.173357 & 0.872491 \\
\hline $\mathrm{H}$ & -5.820265 & -2.013940 & $\odot .214000$ \\
\hline $\mathrm{H}$ & -4.297784 & -3.383997 & -1.226853 \\
\hline $\mathrm{H}$ & -1.966336 & -2.519389 & -1.636595 \\
\hline $\mathrm{H}$ & -2.935118 & 4.471251 & 1.652386 \\
\hline $\mathrm{H}$ & $-\odot .458488$ & 4.535224 & 1.377835 \\
\hline $\mathrm{H}$ & $\odot .743295$ & 2.529140 & $\odot .570996$ \\
\hline $\mathrm{H}$ & 1.148910 & 1.335211 & -2.676084 \\
\hline $\mathrm{H}$ & 3.329060 & 2.422010 & -3.194171 \\
\hline $\mathrm{H}$ & 5.266039 & 2.013656 & -1.649595 \\
\hline $\mathrm{H}$ & 2.059673 & -0.627948 & 4.431244 \\
\hline $\mathrm{H}$ & 2.137480 & -3.092982 & 4.105535 \\
\hline $\mathrm{H}$ & 2.526860 & -4.026514 & 1.833064 \\
\hline $\mathrm{H}$ & -4.177468 & 2.438397 & 1.122388 \\
\hline $\mathrm{H}$ & 2.373163 & ๑.889786 & 2.497743 \\
\hline $\mathrm{Cl}$ & $-\odot .008853$ & -1.279058 & -2.914192 \\
\hline $\mathrm{H}$ & 2.826511 & -2.504052 & -0.101436 \\
\hline $\mathrm{H}$ & $-\odot .04 \odot 723$ & $-\odot .065311$ & $\odot .851065$ \\
\hline $\mathrm{H}$ & -0.000081 & -1.553265 & 0.062996 \\
\hline
\end{tabular}


TS5

B3LYP SCF energy:

-2051.60417044 a.u.

B3LYP enthalpy:

-2051.180883 a.u.

B3LYP free energy:

-2051.262585 a.u.

M06 SCF energy in solution:

-2052.10587480 a.u.

M06 enthalpy in solution:

-2051.682587 a.u.

M06 free energy in solution:

-2051.764289 a.u.

Three lowest frequencies (cm-1):

Imaginary frequency:

$\begin{array}{lll}-106.7092 & 25.5407 & 26.5645\end{array}$

$-106.7092 \mathrm{~cm}-1$

Cartesian coordinates

\begin{tabular}{|c|c|c|c|}
\hline АTOM & $x$ & Y & z \\
\hline $\mathrm{Ru}$ & $\odot .0 \odot 4399$ & -0.785472 & -0.113943 \\
\hline $\mathrm{N}$ & 2.095878 & $-\odot .807680$ & -0.470816 \\
\hline C & 2.849524 & $\odot .212302$ & $\odot .043179$ \\
\hline C & 4.239775 & 0.266129 & -0.251063 \\
\hline C & 4.789211 & $-\odot .772791$ & -1.005036 \\
\hline C & 4.009724 & -1.831596 & -1.458603 \\
\hline C & 2.654838 & -1.805591 & -1.175061 \\
\hline C & 2.059353 & 1.134589 & 0.872862 \\
\hline C & 0.670117 & 0.876261 & $\odot .938919$ \\
\hline C & -0.146392 & 1.649307 & 1.765606 \\
\hline C & ๑.387995 & 2.685310 & 2.526475 \\
\hline C & 1.756695 & 2.958877 & 2.465638 \\
\hline C & 2.580813 & 2.189992 & 1.653859 \\
\hline $\mathrm{N}$ & -2.080511 & -0.776450 & ๑. 292061 \\
\hline C & -2.845049 & $\odot .257428$ & $-\odot .173878$ \\
\hline C & -4.229502 & $\odot .302617$ & $\odot .136301$ \\
\hline C & -4.764346 & -0.756180 & $\odot .875883$ \\
\hline C & -3.973575 & -1.817707 & 1.297506 \\
\hline C & -2.622693 & -1.787572 & $\odot .989462$ \\
\hline C & -2.061451 & 1.212976 & -0.977994 \\
\hline C & $-\odot .673593$ & $\odot .953551$ & -1.075792 \\
\hline C & 0.140580 & 1.774595 & -1.859484 \\
\hline C & -0.392683 & 2.865375 & -2.543121 \\
\hline C & -1.757332 & 3.142421 & -2.444883 \\
\hline C & -2.579012 & 2.325657 & -1.675922 \\
\hline C & -5.172412 & 1.415345 & $-\odot .261929$ \\
\hline $\mathrm{H}$ & -6.149264 & 1.252815 & $\odot .2 \odot 2997$ \\
\hline $\mathrm{H}$ & -5.333088 & 1.457463 & -1.345678 \\
\hline $\mathrm{H}$ & -4.814356 & 2.398731 & 0.061174 \\
\hline C & 5.171042 & 1.375660 & 0.180593 \\
\hline $\mathrm{H}$ & 4.787421 & 2.366152 & -0.085315 \\
\hline $\mathrm{H}$ & 6.141134 & 1.253668 & -0.310446 \\
\hline $\mathrm{H}$ & 5.351836 & 1.367350 & 1.261885 \\
\hline $\mathrm{H}$ & 5.851422 & -0.745625 & -1.234325 \\
\hline
\end{tabular}




$\begin{array}{lrrr}\text { H } & 4.432811 & -2.651453 & -2.028590 \\ \text { H } & 1.983862 & -2.592747 & -1.498791 \\ \text { H } & 2.184878 & 3.761732 & 3.059055 \\ \text { H } & -0.261070 & 3.274359 & 3.169766 \\ \text { H } & -1.208327 & 1.437345 & 1.825821 \\ \text { H } & -1.939529 & -2.563889 & 1.303057 \\ \text { H } & -4.384067 & -2.646901 & 1.863112 \\ \text { H } & -5.823451 & -0.738919 & 1.120064 \\ \text { H } & -2.186596 & 3.988748 & -2.973512 \\ \text { H } & 0.254211 & 3.493512 & -3.150284 \\ \text { H } & 1.203391 & 1.566141 & -1.931570 \\ \text { H } & 3.637160 & 2.407682 & 1.658317 \\ \text { H } & -3.631082 & 2.560127 & -1.641079 \\ \text { Cl } & 0.388931 & -1.696863 & 2.089207 \\ \text { Cl } & -0.423680 & -3.331115 & -1.055555 \\ \text { H } & -0.115932 & -0.814537 & -1.897574 \\ \text { H } & -0.241533 & -1.668206 & -1.796481\end{array}$

TS6

B3LYP SCF energy:

-2051.62652752 a.u.

B3LYP enthalpy:

B3LYP free energy:

-2051.202360 a.u.

-2051.288418 a.u.

M06 SCF energy in solution:

-2052.12223364 a.u.

M06 enthalpy in solution:

-2051.698066 a.u.

M06 free energy in solution: $\quad-2051.784124 \mathrm{a} . \mathrm{u}$.

Three lowest frequencies $(\mathrm{cm}-1): \quad \begin{array}{lll}-290.5996 & 22.7984 & 28.0787\end{array}$

Imaginary frequency:

$-290.5996 \mathrm{~cm}-1$

Cartesian coordinates

\begin{tabular}{lrrr} 
ATOM & X & \multicolumn{1}{c}{ Y } & \multicolumn{1}{l}{$Z$} \\
Ru & 0.210278 & -0.786119 & -0.174084 \\
N & -1.845430 & -0.880117 & 0.283985 \\
C & -2.643240 & $\odot .172999$ & -0.078832 \\
C & -4.038824 & $\odot .110350$ & $\odot .179454$ \\
C & -4.536002 & -1.028592 & 0.820845 \\
C & -3.700335 & -2.077584 & 1.185686 \\
C & -2.350433 & -1.967674 & 0.889006 \\
C & -1.890977 & 1.250898 & -0.764244 \\
C & -0.514980 & 0.965476 & -1.032409 \\
C & 0.221011 & 1.908171 & -1.774192 \\
C & -0.332227 & 3.120650 & -2.189102 \\
C & -1.654277 & 3.419016 & -1.859142 \\
C & -2.425963 & 2.492189 & -1.158741 \\
N & 2.257536 & -0.526957 & -0.520079
\end{tabular}




\begin{tabular}{|c|c|c|c|}
\hline C & 2.651576 & $\odot .386044$ & 0.395720 \\
\hline C & 3.973345 & $\odot .837217$ & 0.486744 \\
\hline C & 4.886978 & $\odot .280398$ & -0.421811 \\
\hline C & 4.476849 & -0.677619 & -1.345919 \\
\hline C & 3.140632 & -1.071313 & -1.366164 \\
\hline C & 1.490903 & $\odot .770669$ & 1.255416 \\
\hline C & 0.852947 & -0.238508 & 2.037114 \\
\hline C & $-\odot .20 \odot \odot 25$ & $\odot .139186$ & 2.915447 \\
\hline C & $-\odot .597348$ & 1.454647 & 3.022742 \\
\hline C & 0.065585 & 2.456112 & 2.274366 \\
\hline C & 1.090686 & 2.126232 & 1.413847 \\
\hline C & 4.411145 & 1.856004 & 1.511382 \\
\hline $\mathrm{H}$ & 3.844931 & 1.761371 & 2.442483 \\
\hline $\mathrm{H}$ & 4.268110 & 2.881434 & 1.147017 \\
\hline $\mathrm{H}$ & 5.474444 & 1.737547 & 1.742813 \\
\hline C & -5.042065 & 1.172451 & -0.209873 \\
\hline $\mathrm{H}$ & -4.901016 & 2.105676 & 0.348324 \\
\hline $\mathrm{H}$ & -6.055220 & ๑. 818566 & 0.003387 \\
\hline $\mathrm{H}$ & -4.994394 & 1.413758 & -1.277121 \\
\hline H & -5.602535 & -1.089468 & 1.022984 \\
\hline $\mathrm{H}$ & -4.081807 & -2.967898 & 1.674463 \\
\hline H & -1.628235 & -2.746894 & 1.113090 \\
\hline H & -2.095858 & 4.366588 & -2.156193 \\
\hline $\mathrm{H}$ & $\odot .265174$ & 3.830964 & -2.756614 \\
\hline $\mathrm{H}$ & 1.258720 & 1.691665 & -2.021054 \\
\hline $\mathrm{H}$ & 2.755891 & -1.822098 & -2.046610 \\
\hline $\mathrm{H}$ & 5.182581 & -1.125799 & -2.037724 \\
\hline $\mathrm{H}$ & 5.928386 & $\odot .590680$ & -0.386162 \\
\hline $\mathrm{H}$ & $-\odot .237879$ & 3.494403 & 2.377134 \\
\hline $\mathrm{H}$ & -1.400036 & 1.731758 & 3.700665 \\
\hline $\mathrm{H}$ & $-\odot .667208$ & -0.630390 & 3.523314 \\
\hline H & -3.443404 & 2.765712 & $-\odot .924803$ \\
\hline $\mathrm{H}$ & 1.584452 & 2.896983 & $\odot .831119$ \\
\hline $\mathrm{Cl}$ & $\odot .795992$ & -3.147830 & 0.620367 \\
\hline $\mathrm{H}$ & 1.302506 & -1.219864 & 2.148837 \\
\hline $\mathrm{Cl}$ & -0.351393 & -1.721272 & -2.625002 \\
\hline $\mathrm{H}$ & $-\odot .462559$ & -0.415701 & -2.202252 \\
\hline
\end{tabular}

TS7

B3LYP SCF energy:

-1131.14532580 a.u.

B3LYP enthalpy:

B3LYP free energy:

-1130.730383 a.u.

-1130.806774 a.u.

M06 SCF energy in solution: $\quad-1131.62256603 \mathrm{a} . \mathrm{u}$.

M06 enthalpy in solution:

-1131.207623 a.u. 
M06 free energy in solution:

Three lowest frequencies (cm-1): Imaginary frequency:
-1131.284014 a.u.

$\begin{array}{lll}-536.8353 \quad 23.4334 & 26.4796\end{array}$

$-536.8353 \mathrm{~cm}-1$

Cartesian coordinates

\begin{tabular}{|c|c|c|c|}
\hline ATOM & $x$ & Y & Z \\
\hline $\mathrm{Ru}$ & $\odot .078907$ & -0.905497 & -0.502146 \\
\hline$N$ & -1.325843 & $\odot .498344$ & -1.081711 \\
\hline C & -2.612345 & ๑. 415920 & $-\odot .590737$ \\
\hline C & -3.599419 & 1.314142 & -1.079983 \\
\hline C & -3.215186 & 2.291696 & -1.998184 \\
\hline C & -1.896052 & 2.390471 & -2.431689 \\
\hline C & $-\odot .985230$ & 1.464443 & -1.954508 \\
\hline C & -2.759536 & $-\odot .640273$ & $\odot .419997$ \\
\hline C & -1.575870 & -1.401617 & 0.605943 \\
\hline C & -1.574707 & -2.420392 & 1.568521 \\
\hline C & -2.699665 & -2.676244 & 2.352359 \\
\hline C & -3.863166 & -1.920184 & 2.168767 \\
\hline C & -3.898249 & $-\odot .911964$ & 1.209969 \\
\hline $\mathrm{N}$ & ๑. 974616 & $\odot .648682$ & $\odot .877845$ \\
\hline C & 2.303441 & $\odot .889043$ & $\odot .701288$ \\
\hline C & 2.902572 & 2.045592 & 1.255967 \\
\hline C & 2.100874 & 2.868502 & 2.056347 \\
\hline C & ๑. 763966 & 2.563656 & 2.289548 \\
\hline C & ๑. 234828 & 1.443925 & 1.657665 \\
\hline C & 2.967965 & -0.158058 & -0.109135 \\
\hline C & 2.131828 & -0.964928 & -0.937397 \\
\hline C & 2.718266 & -2.030796 & -1.639923 \\
\hline C & 4.077837 & -2.332228 & -1.511286 \\
\hline C & 4.883438 & -1.550299 & $-\odot .685687$ \\
\hline C & 4.331162 & -0.473455 & ๑. 011071 \\
\hline C & 4.330376 & 2.471692 & $\odot .997158$ \\
\hline $\mathrm{H}$ & 4.429989 & 3.551643 & 1.148148 \\
\hline $\mathrm{H}$ & 4.648879 & 2.239984 & $-\odot .023235$ \\
\hline $\mathrm{H}$ & 5.039441 & 1.986906 & 1.680367 \\
\hline C & -5.054518 & 1.263775 & -0.676684 \\
\hline $\mathrm{H}$ & -5.482454 & ๑. 265117 & -0.812393 \\
\hline $\mathrm{H}$ & -5.634004 & 1.961325 & -1.289134 \\
\hline $\mathrm{H}$ & -5.203931 & 1.547532 & $\odot .371864$ \\
\hline $\mathrm{H}$ & -3.965535 & 2.980927 & -2.377026 \\
\hline $\mathrm{H}$ & -1.580370 & 3.150986 & -3.137702 \\
\hline $\mathrm{H}$ & $\odot . \odot 49466$ & 1.463356 & -2.272043 \\
\hline $\mathrm{H}$ & -4.745331 & -2.117727 & 2.772064 \\
\hline $\mathrm{H}$ & -2.678254 & -3.466675 & 3.099815 \\
\hline $\mathrm{H}$ & $-\odot .681350$ & -3.030450 & 1.704793 \\
\hline $\mathrm{H}$ & $-\odot .80900 \odot$ & 1.164404 & 1.762869 \\
\hline
\end{tabular}




$\begin{array}{rrrc}\text { H } & 0.139721 & 3.188942 & 2.919692 \\ \mathrm{H} & 2.538072 & 3.764606 & 2.490837 \\ \mathrm{H} & 5.939840 & -1.778484 & -0.571053 \\ \mathrm{H} & 4.504453 & -3.169536 & -2.058477 \\ \mathrm{H} & 2.095185 & -2.632794 & -2.294850 \\ \mathrm{H} & -4.813503 & -0.347162 & 1.105541 \\ \mathrm{H} & 4.967924 & 0.080454 & 0.689927 \\ \mathrm{H} & 0.714832 & -0.642301 & -1.966663 \\ \mathrm{H} & -0.563044 & -2.002879 & -1.498178\end{array}$

TS8

B3LYP SCF energy:

-1131.16465473 a.u.

B3LYP enthalpy:

-1130.747135 a.u.

B3LYP free energy:

-1130.823053 a.u.

M06 SCF energy in solution:

-1131.64302722 a.u.

M06 enthalpy in solution:

-1131.225507 a.u.

M06 free energy in solution:

-1131.301425 a.u.

Three lowest frequencies (cm-1): $\begin{array}{llll}-281.3758 & 24.5232 & 31.8017\end{array}$

Imaginary frequency:

$-281.3758 \mathrm{~cm}-1$

Cartesian coordinates

\begin{tabular}{|c|c|c|c|}
\hline ATOM & $x$ & Y & Z \\
\hline $\mathrm{Ru}$ & $-\odot .074426$ & $-\odot .208795$ & -0.050024 \\
\hline $\mathrm{N}$ & -1.857099 & -1.258627 & -0.223056 \\
\hline C & -2.909787 & -0.388151 & -0.105087 \\
\hline C & -4.242053 & $-\odot .787200$ & -0.332205 \\
\hline C & -4.463213 & -2.114681 & $-\odot .714098$ \\
\hline C & -3.387310 & -2.990310 & $-\odot .862937$ \\
\hline C & -2.106942 & -2.529786 & -0.604118 \\
\hline C & -2.481359 & $\odot .969754$ & 0.311573 \\
\hline C & -1.460691 & 1.096502 & 1.296035 \\
\hline C & -1.090381 & 2.372867 & 1.768518 \\
\hline C & -1.668593 & 3.515956 & 1.237323 \\
\hline C & -2.634236 & 3.399569 & $\odot .220682$ \\
\hline C & -3.031528 & 2.149015 & $-\odot .236169$ \\
\hline $\mathrm{N}$ & 1.589158 & 1.037124 & $-\odot .238999$ \\
\hline C & 2.839334 & $\odot .464653$ & $-\odot .217226$ \\
\hline C & 3.982327 & 1.225294 & $-\odot .577881$ \\
\hline C & 3.802231 & 2.571308 & -0.898480 \\
\hline C & 2.531051 & 3.146351 & $-\odot .879867$ \\
\hline C & 1.453894 & 2.341196 & $-\odot .555858$ \\
\hline C & 2.785452 & -0.956213 & $\odot .132090$ \\
\hline C & 1.482577 & -1.561394 & ๑.018067 \\
\hline C & 1.379832 & -2.933203 & 0.364438 \\
\hline
\end{tabular}




$\begin{array}{lrrr}\text { C } & 2.461429 & -3.653105 & 0.853917 \\ \text { C } & 3.710599 & -3.037460 & 1.002255 \\ \text { C } & 3.862402 & -1.702284 & 0.641264 \\ \text { C } & 5.377164 & 0.651714 & -0.683191 \\ \text { H } & 6.011136 & 1.321311 & -1.273292 \\ \text { H } & 5.378855 & -0.330815 & -1.165306 \\ \text { H } & 5.856331 & 0.536169 & 0.297275 \\ \text { C } & -5.426307 & 0.133986 & -0.143171 \\ \text { H } & -5.262397 & 0.853188 & 0.664232 \\ \text { H } & -6.320974 & -0.450242 & 0.095704 \\ \text { H } & -5.650302 & 0.708249 & -1.051151 \\ \text { H } & -5.479763 & -2.460346 & -0.882405 \\ \text { H } & -3.538535 & -4.021680 & -1.165628 \\ \text { H } & -1.240807 & -3.169075 & -0.716390 \\ \text { H } & -3.071662 & 4.293449 & -0.215902 \\ \text { H } & -1.373306 & 4.498230 & 1.596384 \\ \text { H } & -0.334852 & 2.446272 & 2.545753 \\ \text { H } & 0.439525 & 2.722986 & -0.546215 \\ \text { H } & 2.376291 & 4.190452 & -1.132446 \\ \text { H } & 4.666129 & 3.167088 & -1.182663 \\ \text { H } & 4.555712 & -3.591394 & 1.401296 \\ \text { H } & 2.331951 & -4.695414 & 1.137425 \\ \text { H } & 0.419477 & -3.430737 & 0.281472 \\ \text { H } & -3.758705 & 2.079417 & -1.038311 \\ \text { H } & 4.822581 & -1.231381 & 0.806864 \\ \text { H } & 0.858202 & -1.212871 & -1.097122 \\ \text { H } & -1.129901 & 0.208389 & 1.860605\end{array}$

TS9

B3LYP SCF energy:

-2166.05685467 a.u.

B3LYP enthalpy:

-2165.259516 a.u.

B3LYP free energy:

-2165.379662 a.u.

M06 SCF energy in solution: $\quad-2166.04703904 \mathrm{a} . \mathrm{u}$.

M06 enthalpy in solution: $\quad-2165.249700$ a.u.

M06 free energy in solution: $\quad-2165.369846$ a.u.

Three lowest frequencies $(\mathrm{cm}-1): \begin{array}{llll}-227.0820 & 21.8337 & 24.2490\end{array}$

Imaginary frequency: $\quad-227.0820 \mathrm{~cm}-1$

Cartesian coordinates

$\begin{array}{lccl}\text { ATOM } & \mathrm{X} & \mathrm{Y} & \mathrm{Z} \\ \mathrm{Ru} & 0.538996 & -0.019174 & -0.043865 \\ \mathrm{~N} & 0.494940 & -1.863015 & -1.034667 \\ \mathrm{C} & 1.514790 & -2.725150 & -0.707427 \\ \mathrm{C} & 1.715892 & -3.925426 & -1.434612\end{array}$




\begin{tabular}{|c|c|c|c|}
\hline C & $\odot .802547$ & -4.243074 & -2.443140 \\
\hline C & $-\odot .267898$ & -3.393981 & -2.718768 \\
\hline C & $-\odot .377282$ & -2.213450 & -2.000363 \\
\hline C & 2.356606 & -2.248007 & 0.404713 \\
\hline C & 2.504838 & $-\odot .827265$ & 0.614816 \\
\hline C & 3.231695 & -0.447593 & 1.776816 \\
\hline C & 3.747562 & -1.361694 & 2.681798 \\
\hline C & 3.573976 & -2.736138 & 2.467959 \\
\hline C & 2.881186 & -3.158286 & 1.337759 \\
\hline $\mathrm{N}$ & $\odot .962788$ & 1.756246 & 0.993804 \\
\hline C & 2.180573 & 2.342879 & 0.705318 \\
\hline C & 2.636578 & 3.450612 & 1.485626 \\
\hline C & 1.805032 & 3.940942 & 2.487184 \\
\hline C & $\odot .557167$ & 3.359540 & 2.733591 \\
\hline C & $\odot .187298$ & 2.267757 & 1.969248 \\
\hline C & 2.905076 & 1.674523 & -0.366626 \\
\hline C & 2.526662 & $\odot .285087$ & $-\odot .661919$ \\
\hline C & 3.156829 & -0.263966 & -1.842566 \\
\hline C & 4.053087 & 0.432636 & -2.623074 \\
\hline C & 4.403841 & 1.759814 & -2.303034 \\
\hline C & 3.821649 & 2.353870 & -1.192839 \\
\hline C & 4.008282 & 4.072461 & 1.341402 \\
\hline $\mathrm{H}$ & 4.274394 & 4.601731 & 2.262677 \\
\hline $\mathrm{H}$ & 4.057427 & 4.805652 & $\odot .526093$ \\
\hline $\mathrm{H}$ & 4.777942 & 3.319678 & 1.143939 \\
\hline C & 2.900767 & -4.844677 & -1.227667 \\
\hline H & 3.795623 & -4.295774 & -0.922067 \\
\hline $\mathrm{H}$ & 3.124495 & -5.375981 & -2.159085 \\
\hline $\mathrm{H}$ & 2.710431 & -5.608422 & -0.462668 \\
\hline $\mathrm{H}$ & 0.945710 & -5.151870 & -3.022907 \\
\hline $\mathrm{H}$ & -0.994100 & -3.626635 & -3.491160 \\
\hline H & -1.175192 & -1.507916 & -2.190201 \\
\hline $\mathrm{H}$ & 3.961061 & -3.463962 & 3.175743 \\
\hline $\mathrm{H}$ & 4.288676 & -1.008686 & 3.557446 \\
\hline $\mathrm{H}$ & 3.408965 & 0.608813 & 1.952332 \\
\hline $\mathrm{H}$ & $-\odot .757005$ & 1.763104 & 2.125315 \\
\hline H & -0.103073 & 3.731634 & 3.510243 \\
\hline H & 2.148698 & 4.772614 & 3.098310 \\
\hline $\mathrm{H}$ & 5.094175 & 2.319942 & -2.927289 \\
\hline $\mathrm{H}$ & 4.486230 & -0.045902 & -3.500101 \\
\hline H & 2.933783 & -1.290387 & -2.116965 \\
\hline H & 2.686805 & -4.217823 & 1.212338 \\
\hline H & 4.028149 & 3.401328 & -1.009602 \\
\hline $\mathrm{N}$ & $-\odot .580720$ & 1.010246 & -1.707787 \\
\hline C & -1.644211 & 1.860231 & -1.656629 \\
\hline C & -2.104284 & 2.570944 & -2.791560 \\
\hline
\end{tabular}




\begin{tabular}{|c|c|c|c|}
\hline C & -1.439902 & 2.382298 & $-4.0 \odot 4 \odot 42$ \\
\hline C & -0.350787 & 1.516948 & -4.059251 \\
\hline C & ๑.039458 & $\odot .862248$ & -2.902491 \\
\hline C & -2.342856 & 2.062048 & -0.348308 \\
\hline C & -3.081366 & 1.041015 & ๑. 288085 \\
\hline C & -3.743738 & 1.331152 & 1.490942 \\
\hline C & -3.701638 & 2.605225 & 2.057558 \\
\hline C & -2.982657 & 3.616869 & 1.422061 \\
\hline C & -2.308634 & 3.338551 & $\odot .234228$ \\
\hline$N$ & -0.949558 & $-\odot .847792$ & 1.718292 \\
\hline C & -2.154020 & -1.467655 & 1.662851 \\
\hline C & -2.740970 & -2.092951 & 2.789750 \\
\hline C & -2.027433 & -2.074161 & 3.990051 \\
\hline C & $-\odot .781664$ & -1.456456 & 4.045106 \\
\hline C & -0.289554 & -0.857326 & 2.893466 \\
\hline C & -2.862612 & -1.490741 & 0.345304 \\
\hline C & -3.259079 & -0.310658 & -0.320419 \\
\hline C & -3.925905 & -0.418016 & -1.550813 \\
\hline C & -4.211564 & -1.659496 & -2.119303 \\
\hline C & -3.825479 & -2.824899 & -1.457729 \\
\hline C & -3.153904 & -2.732778 & -0.239912 \\
\hline C & -4.112198 & -2.733786 & 2.753971 \\
\hline $\mathrm{H}$ & -4.527219 & -2.793128 & 3.765707 \\
\hline $\mathrm{H}$ & -4.081649 & -3.754672 & 2.354596 \\
\hline $\mathrm{H}$ & -4.812681 & -2.171643 & 2.129133 \\
\hline C & -3.305101 & 3.492484 & -2.744342 \\
\hline $\mathrm{H}$ & -4.114838 & 3.085617 & -2.130793 \\
\hline $\mathrm{H}$ & -3.691827 & 3.659330 & -3.755177 \\
\hline $\mathrm{H}$ & -3.055213 & 4.473732 & -2.323772 \\
\hline $\mathrm{H}$ & -1.779126 & 2.907278 & -4.893645 \\
\hline $\mathrm{H}$ & 0.197658 & 1.349970 & -4.981185 \\
\hline $\mathrm{H}$ & $0.9 \odot 4571$ & $\odot .210901$ & $-2.9 \odot 4281$ \\
\hline $\mathrm{H}$ & -2.934843 & 4.613935 & 1.851209 \\
\hline $\mathrm{H}$ & -4.236115 & $2.8 \odot 4876$ & 2.982497 \\
\hline $\mathrm{H}$ & -4.322680 & $\odot .548102$ & 1.972188 \\
\hline $\mathrm{H}$ & 0.680747 & -0.374229 & 2.893226 \\
\hline $\mathrm{H}$ & $-\odot .199943$ & -1.430207 & 4.961319 \\
\hline $\mathrm{H}$ & -2.455107 & -2.536865 & 4.876515 \\
\hline $\mathrm{H}$ & -4.035696 & $-3.8 \odot \odot \odot \odot \odot$ & -1.888704 \\
\hline $\mathrm{H}$ & -4.741109 & -1.712476 & -3.066843 \\
\hline $\mathrm{H}$ & -4.247573 & $\odot .489022$ & -2.053658 \\
\hline $\mathrm{H}$ & -1.722929 & 4.116898 & $-\odot .246 \odot 37$ \\
\hline $\mathrm{H}$ & -2.825527 & -3.638742 & $\odot .261240$ \\
\hline
\end{tabular}




\section{References}

${ }^{1}$ K. L. Hull, E. L. Lanni, M. S. Sanford, J. Am. Chem. Soc. 2006, 128, 14047-14049.

2 X. Guo, G. Deng, C.-J. Li, Adv. Synth. Catal. 2009, 351, 2071-2074.

${ }^{3}$ M. Iyoda, M. Sakaitan, H. Otsuka, M. Oda, Chem. Lett. 1985, 14, 127-130.

4 A. J. Paterson, S. St John-Campbell, M. F. Mahon, N. J. Press, C. G. Frost, Chem. Commun. 2015, 51, 12807-12810.

5 W. Liu, Y. Zhu, C.-J. Li, Synthesis 2016, 48, 1616-1621.

${ }^{6}$ F. Saito, H. Aiso, T. Kochi, F. Kakiuchi, Organometallics 2014, 33, 6704-6707.

7 J. P. H. Charmant, I. A. Fallis, N. J. Hunt, G. C. Lloyd-Jones, M. Murray, T. Nowak, J. Chem. Soc., Dalton Trans. 2000, $1723-1732$.

${ }^{8}$ P. B. Arockiam, C. Fischmeister, C. Bruneau, P. H. Dixneuf, Green Chem. 2011, 13, 3075-3078.

${ }^{9}$ G. R. Kiel, A. E. Samkian, A. Nicolay, R. J. Witzke, T. D. Tilley, J. Am. Chem. Soc. 2018, 140, 2450-2454.

${ }^{10}$ P. Yu, B. Morandi, Angew. Chem. Int. Ed. 2017, 56, 15693-15697.

11 V. P. Reddy, R. Qiu, T. Iwasaki, N. Kambe, Org. Lett. 2013, 15, 1290-1293.

12 L.-Y. Xi, R.-Y. Zhang, S. Liang, S.-Y. Chen, X.-Q. Yu, Org. Lett. 2014, 16, 5269-5271.

13 T. Nokami, Y. Tomida, T. Kamei, K. Itami, J.-i. Yoshida, Org. Lett. 2006, 8, 729-731.

${ }^{14}$ H. Liang, T. Hao, C. Yin, X. Yang, H. Fu, X. Zheng, R. Li, D. Xiao, H. Chen, Eur. J. Inorg. Chem. 2017, $2017,4149-4157$.

15 G.-L. Li, K. K.-Y. Kung, M.-K. Wong, Chem. Commun. 2012, 48, 4112-4114.

16 Z. Fan, H. Lu, Z. Cheng, A. Zhang, Chem. Commun. 2018, 54, 6008-6011.

17 J. K. Laha, K. P. Jethava, S. Patel, Org. Lett. 2015, 17, 5890-5893.

18 A. B. Pawar, S. Chang, Org. Lett. 2015, 17, 660-663.

19 S. Roesner, S. L. Buchwald, Angew. Chem. Int. Ed. 2016, 55, 10463-10467.

${ }^{20}$ K. M. Liu, L. Y. Liao, X. F. Duan, Chem. Commun. 2015, 51, 1124-1127.

${ }^{21}$ D. Hackenberger, P. Weber, D. C. Blakemore, L. J. Goossen, J. Org. Chem. 2017, 82, 3917-3925.

${ }^{22}$ M. J. Iglesias, A. Prieto, M. C. Nicasio, Org. Lett. 2012, 14, 4318-4321.

${ }^{23}$ D. Toummini, A. Tlili, J. Bergès, F. Ouazzani, M. Taillefer, Chem. Eur. J. 2014, 20, 14619-14623.

24 C. Hansch, A. Leo, R. W. Taft, Chem. Rev. 1991, 91, 165-195.

25 E. Solari, S. Gauthier, R. Scopelliti, K. Severin, Organometallics 2009, 28, 4519-4526.

${ }^{26}$ B. Demerseman, M. D. Mbaye, D. Sémeril, L. Toupet, C. Bruneau, P. H. Dixneuf, Eur. J. Inorg. Chem. 2006, 2006, 1174-1181.

27 A. Kaithal, B. Chatterjee, C. Gunanathan, Org. Lett. 2016, 18, 3402-3405.

${ }^{28}$ L. Fang, L. Chen, J. Yu, L. Wang, Eur. J. Org. Chem. 2015, 2015, 1910-1914.

${ }^{29}$ Frisch, M. J.; Trucks, G. W.; Schlegel, H. B.; Scuseria, G. E.; Robb, M. A.; Cheeseman, J. R.; Scalmani, G.; Barone, V.; Mennucci, B.; Petersson, G. A.; Nakatsuji, H.; Caricato, M.; Li, X.; Hratchian, H. P.; Izmaylov, A. F.; Bloino, J.; Zheng, G.; Sonnenberg, J. L.; Hada, M.; Ehara, M.; Toyota, K.; Fukuda, R.; Hasegawa, J.; Ishida, M.; Nakajima, T.; Honda, Y.; Kitao, O.; Nakai, H.; Vreven, T.; Montgomery, J. A., Jr.; Peralta, J. E.; Ogliaro, F.; Bearpark, M.; Heyd, J. J.; Brothers, E.; Kudin, K. N.; Staroverov, V. N.; Kobayashi, R.; Normand, J.; Raghavachari, K.; Rendell, A.; Burant, J. C.; Iyengar, S. S.; Tomasi, J.; Cossi, M.; Rega, N.; Millam, N. J.; Klene, M.; Knox, J. E.; Cross, J. B.; Bakken, V.; Adamo, C.; Jaramillo, J.; Gomperts, R.; Stratmann, R. E.; Yazyev, O.; Austin, A. J.; Cammi, R.; Pomelli, C.; Ochterski, J. W.; Martin, R. L.; Morokuma, K.; 
Zakrzewski, V. G.; Voth, G. A.; Salvador, P.; Dannenberg, J. J.; Dapprich, S.; Daniels, A. D.; Farkas, Ö.; Foresman, J. B.; Ortiz, J. V.; Cioslowski, J.; Fox, D. J. Gaussian 09, Revision D.01; Gaussian, Inc.: Wallingford, CT, 2009.

${ }^{30}$ Zhao, Y.; Truhlar, D. G. Theor. Chem. Acc. 2008, 120, 215-241.

${ }^{31}$ Marenich, A. V.; Cramer, C. J.; Truhlar, D. G. J. Phys. Chem. B 2009, 113, 6378-6396.

32 4.CYLview, 1.0b; Legault, C. Y., Université de Sherbrooke, 2009 (http://www.cylview.org). 
9. NMR spectra
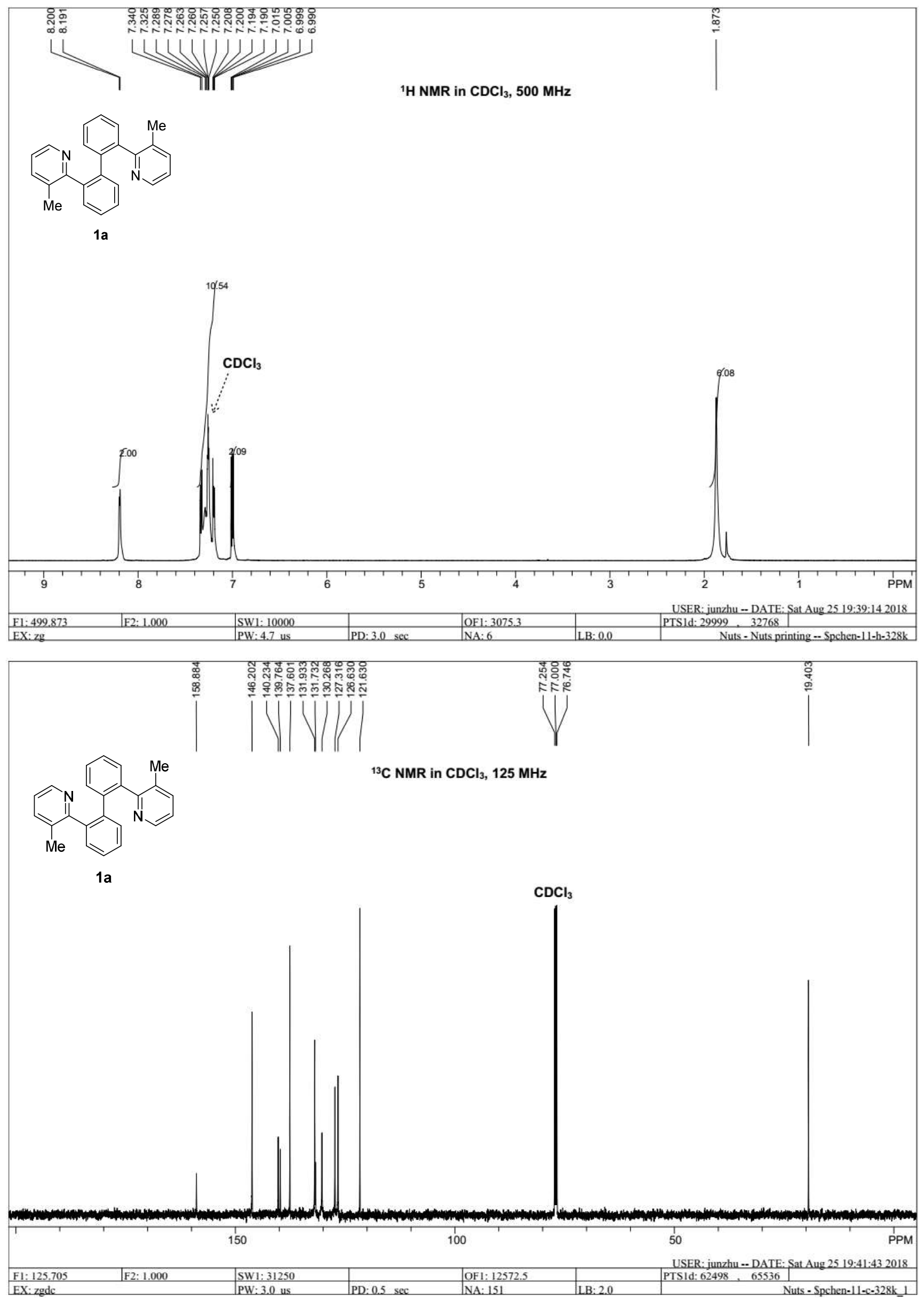

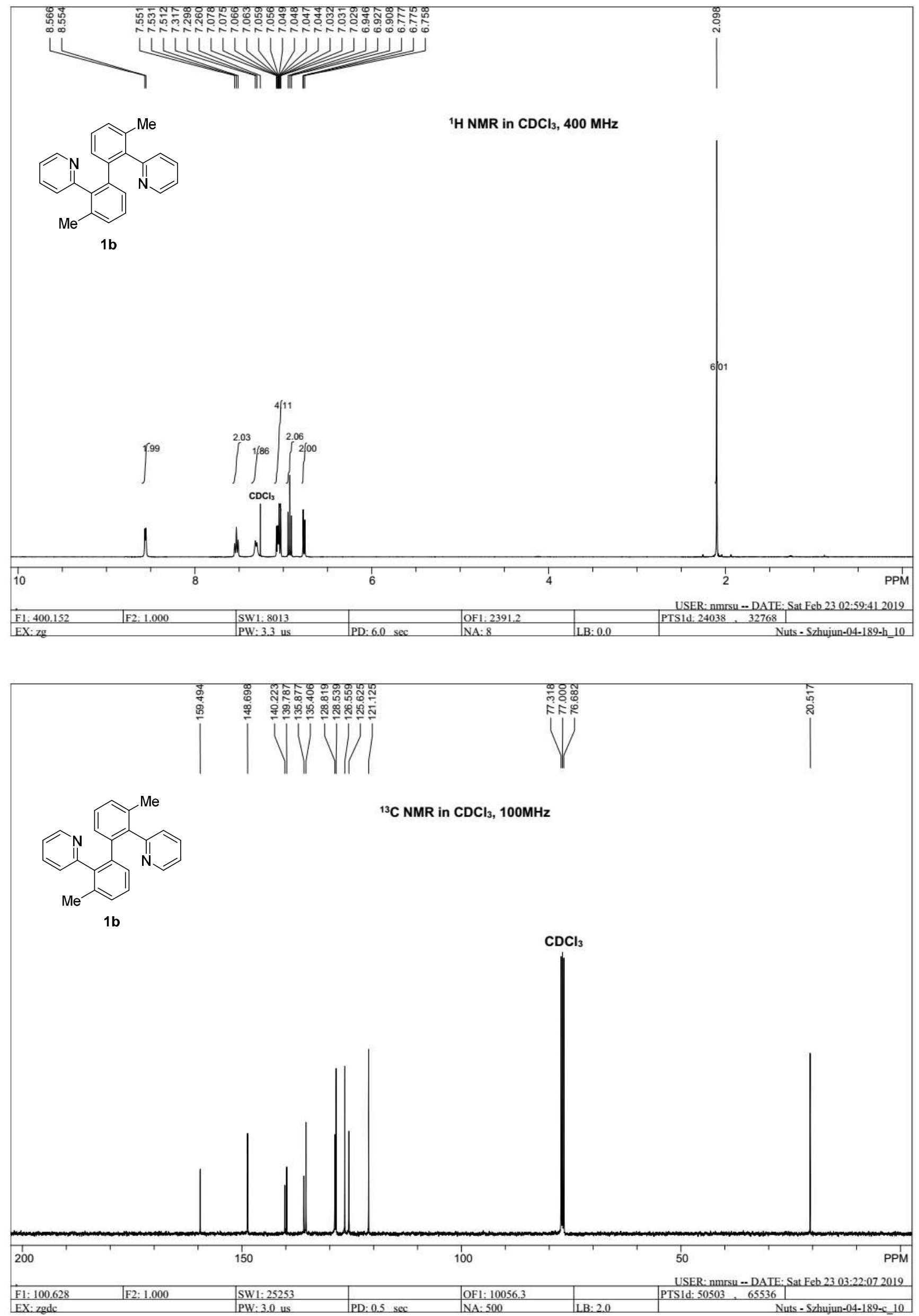

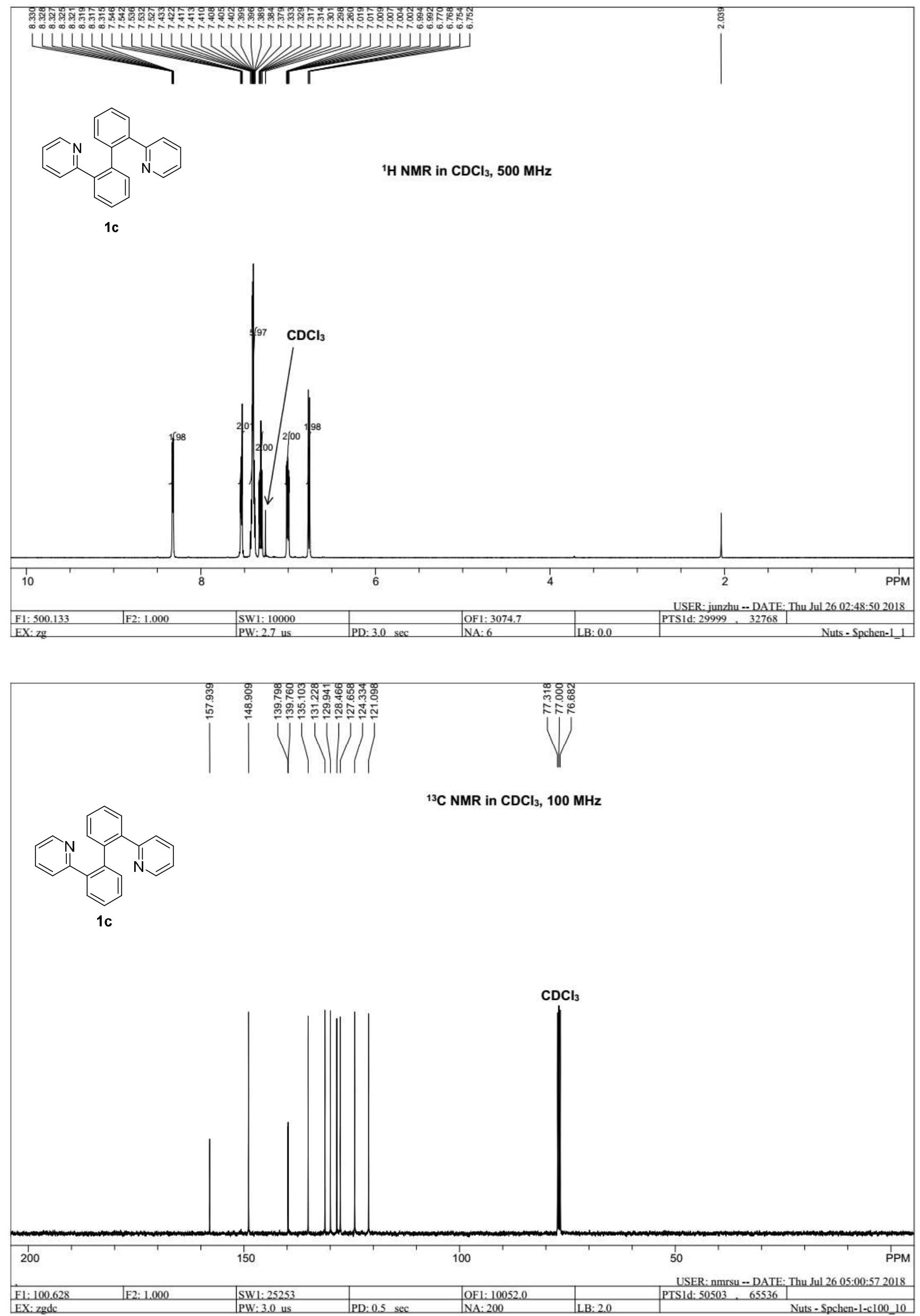

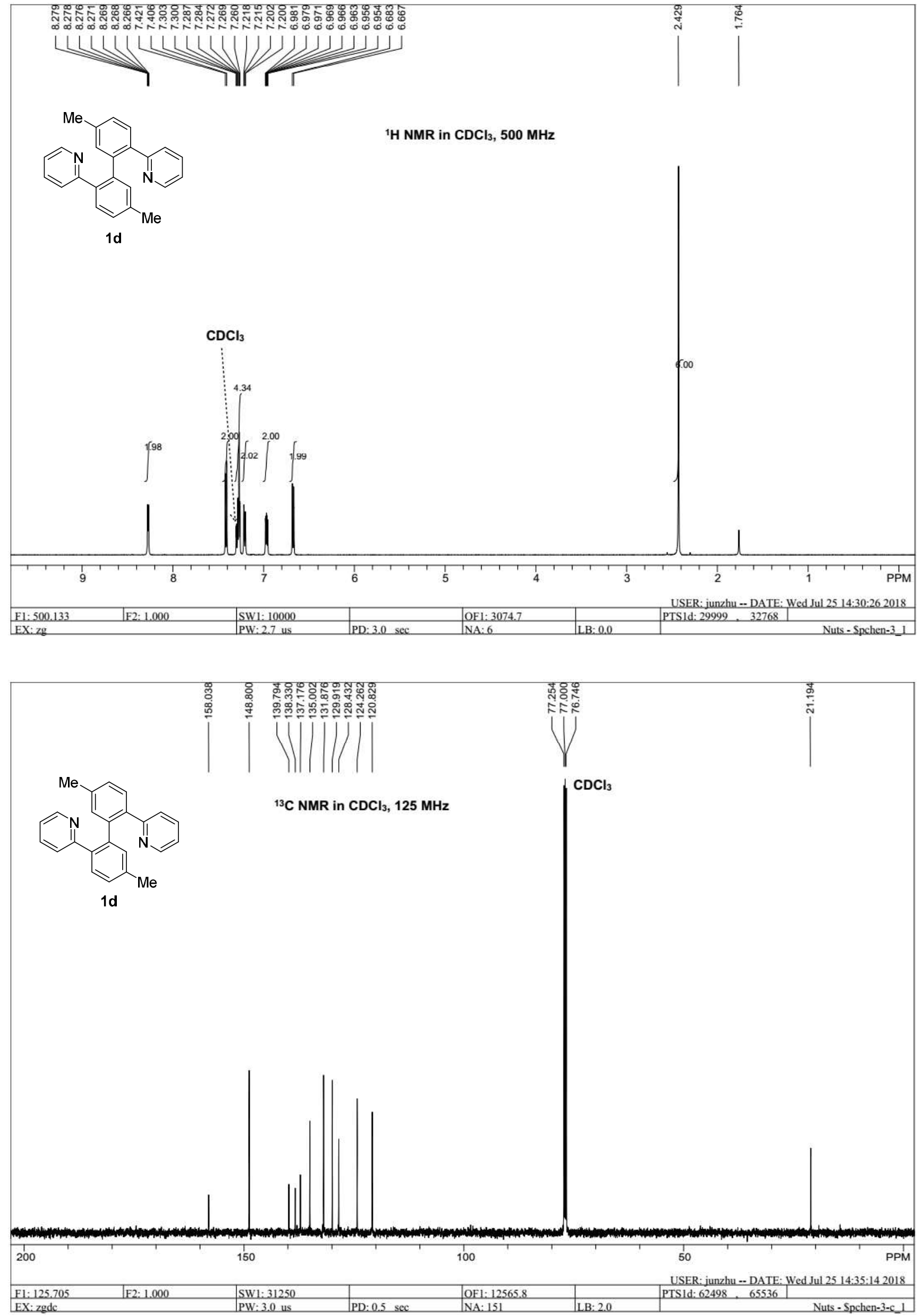

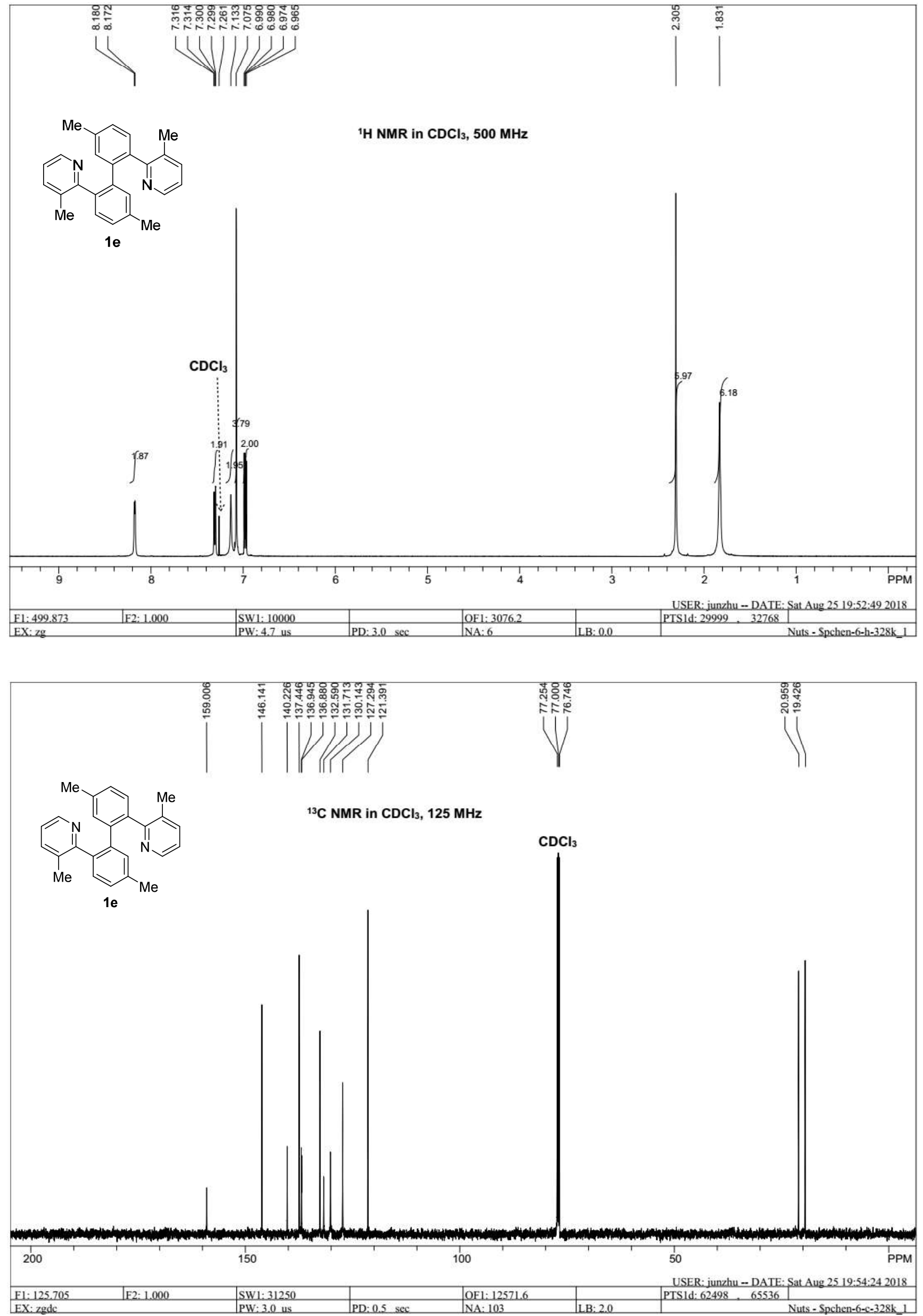

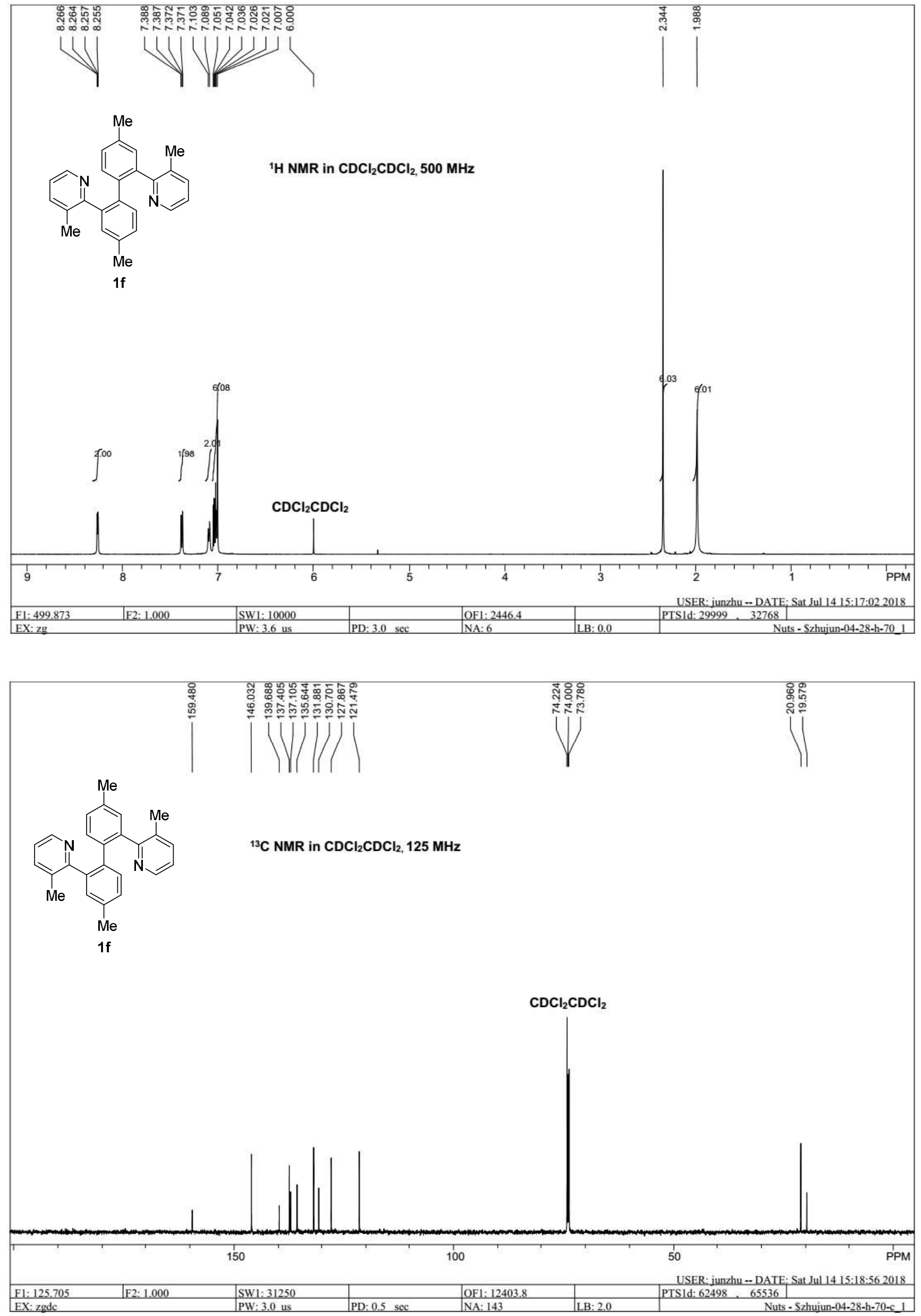

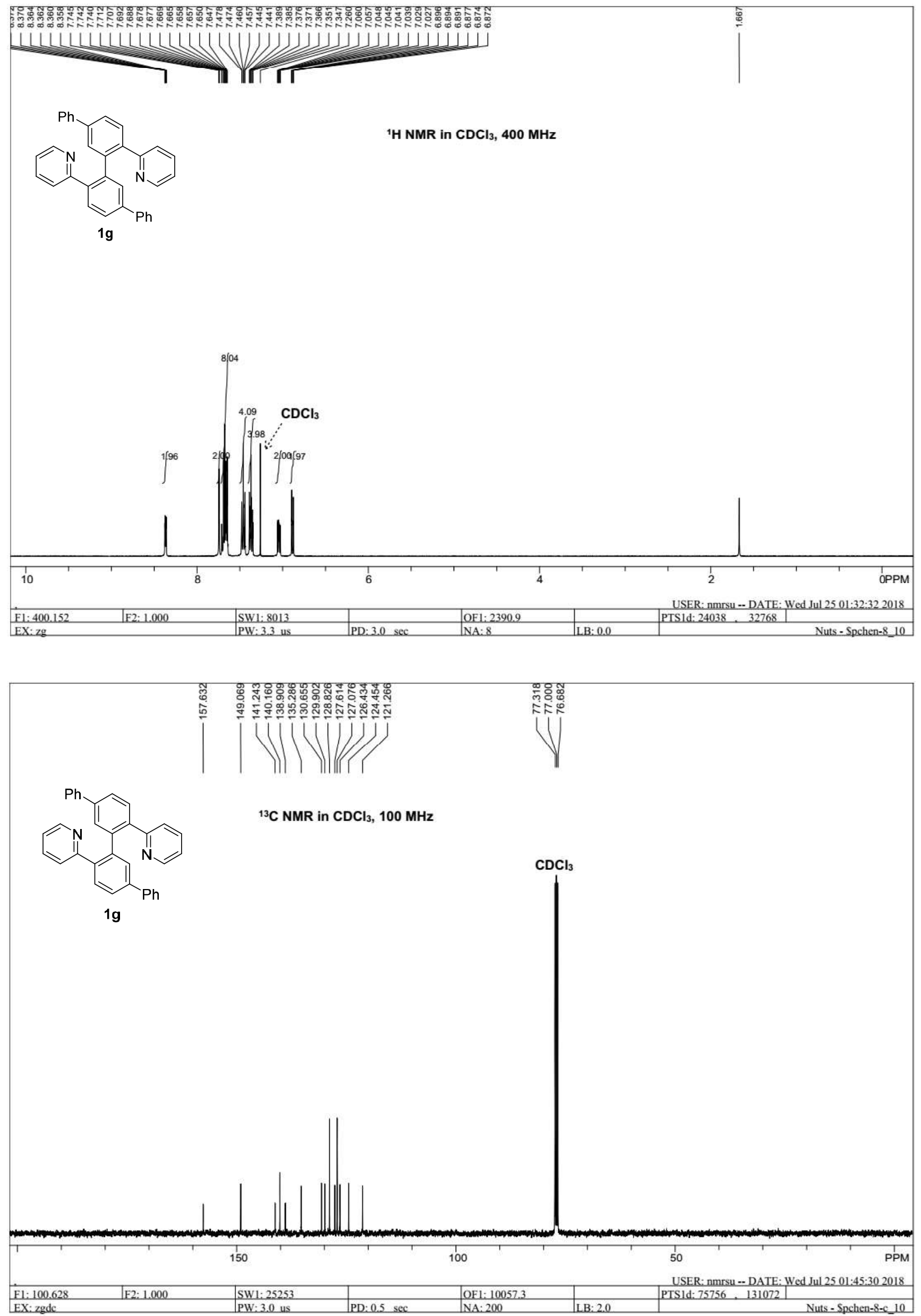
<smiles>c1ccc(-c2ccc(-c3ccccn3)c(-c3ccc(-c4ccco4)cc3)c2)nc1</smiles>

${ }^{1} \mathrm{H} \mathrm{NMR}$ in $\mathrm{CDCl}_{3}, 500 \mathrm{MHz}$
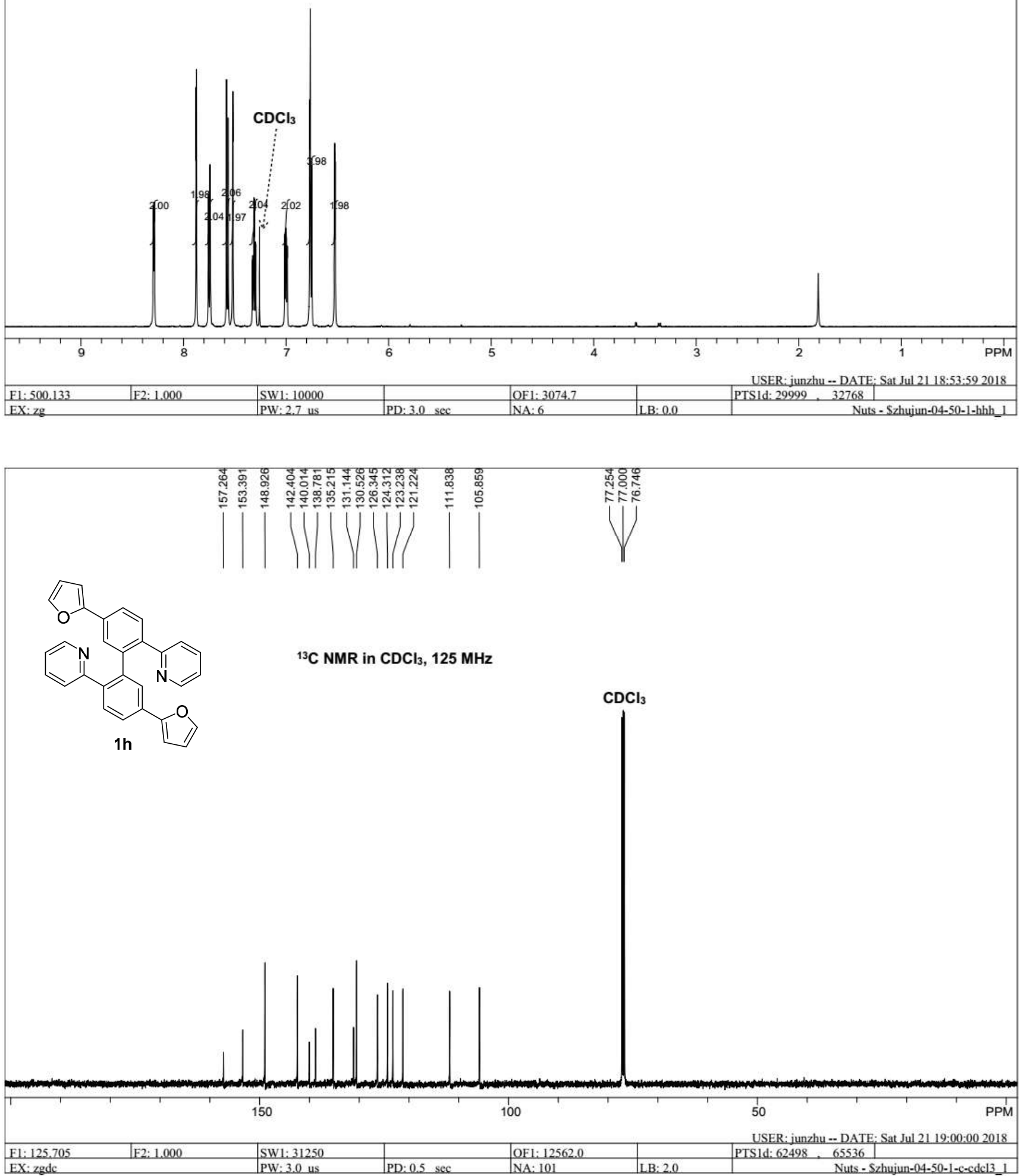

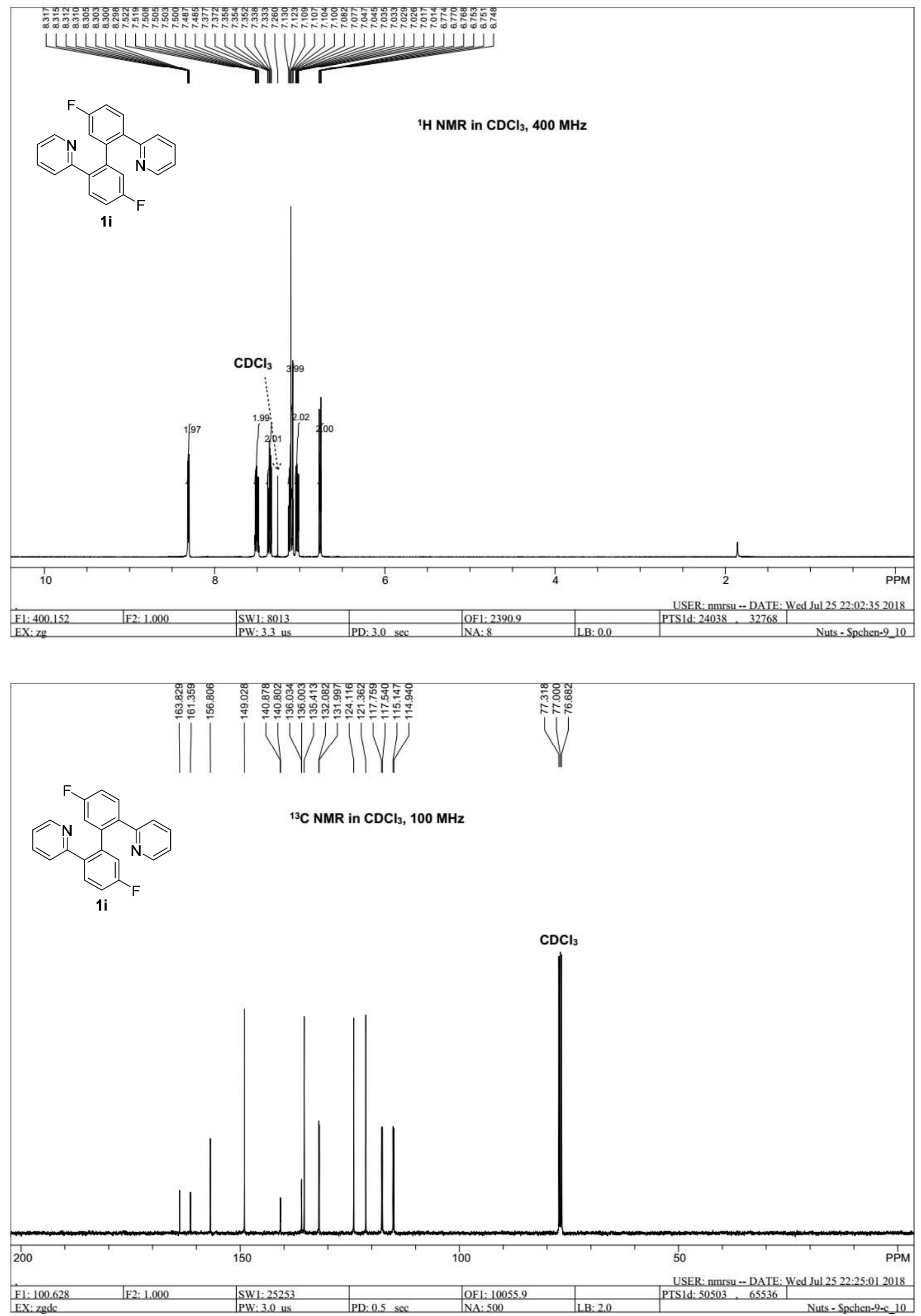


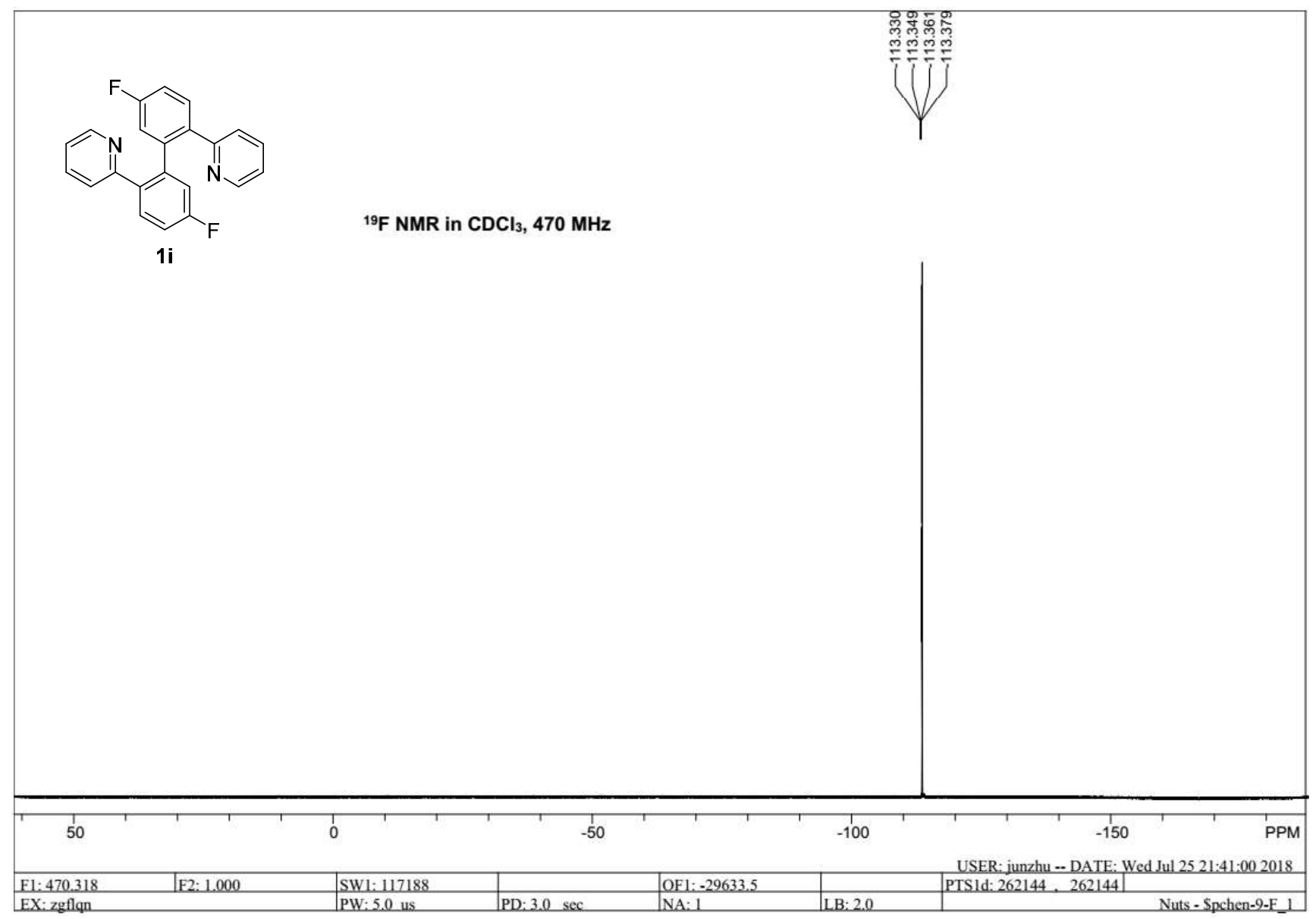



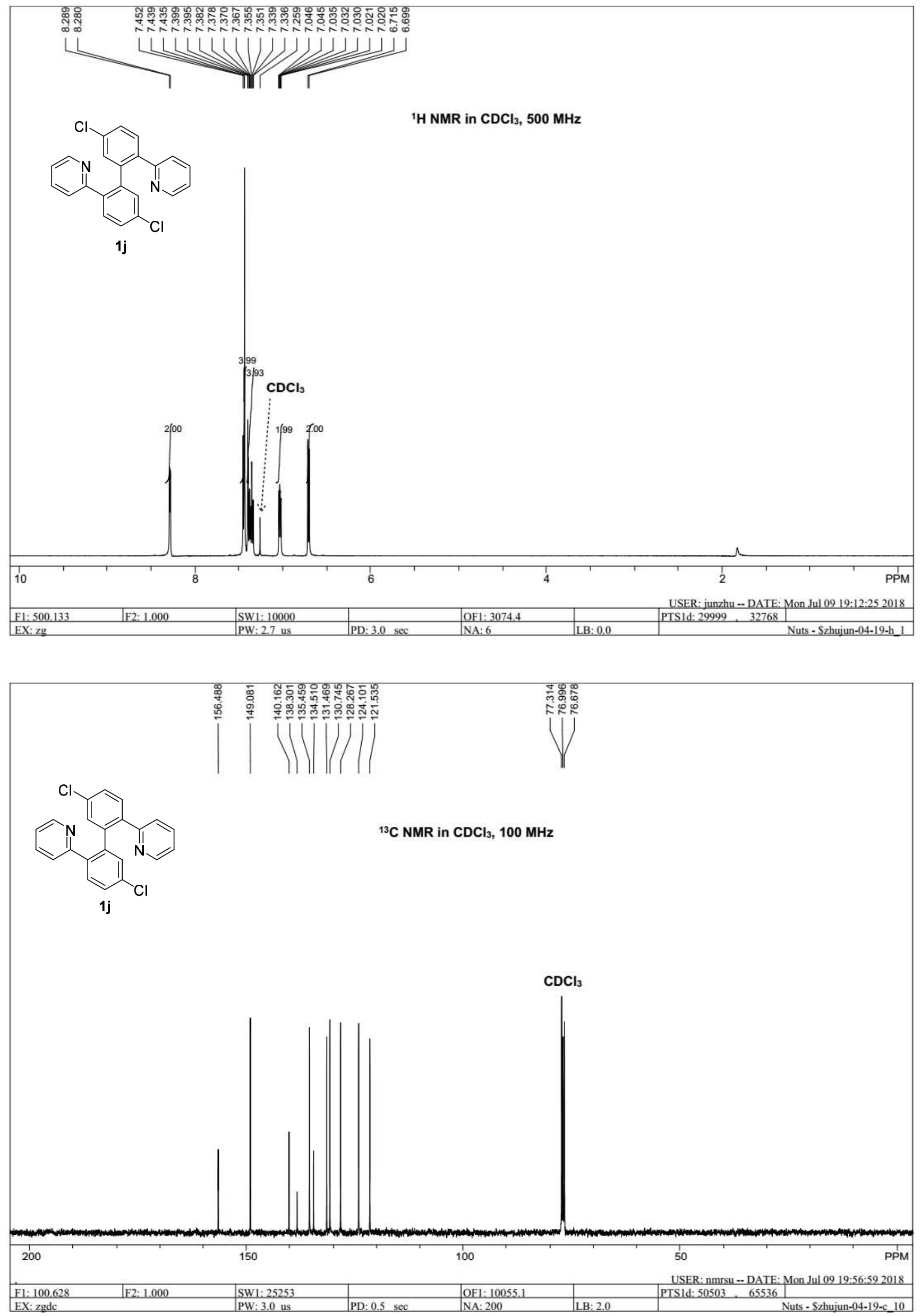

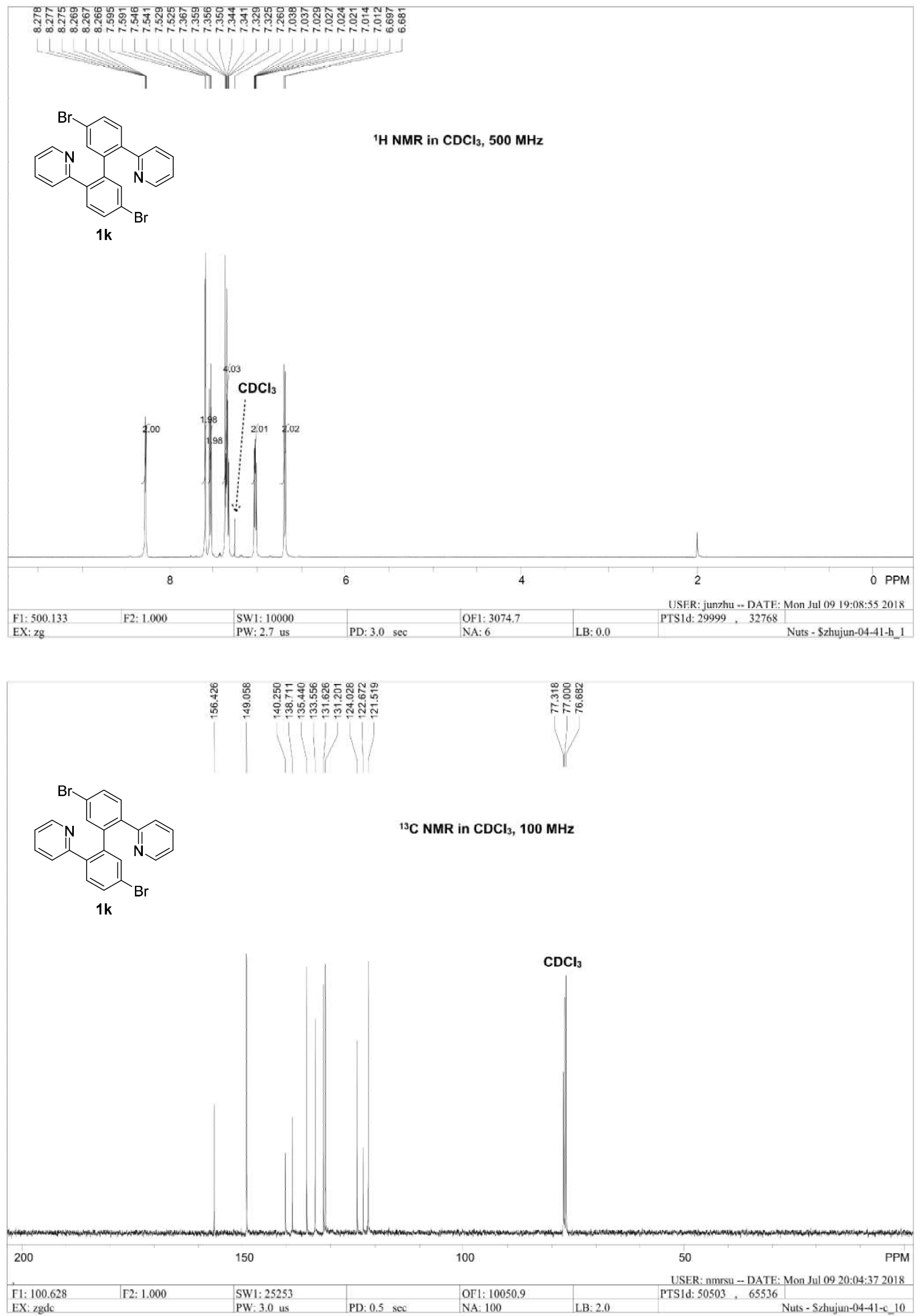

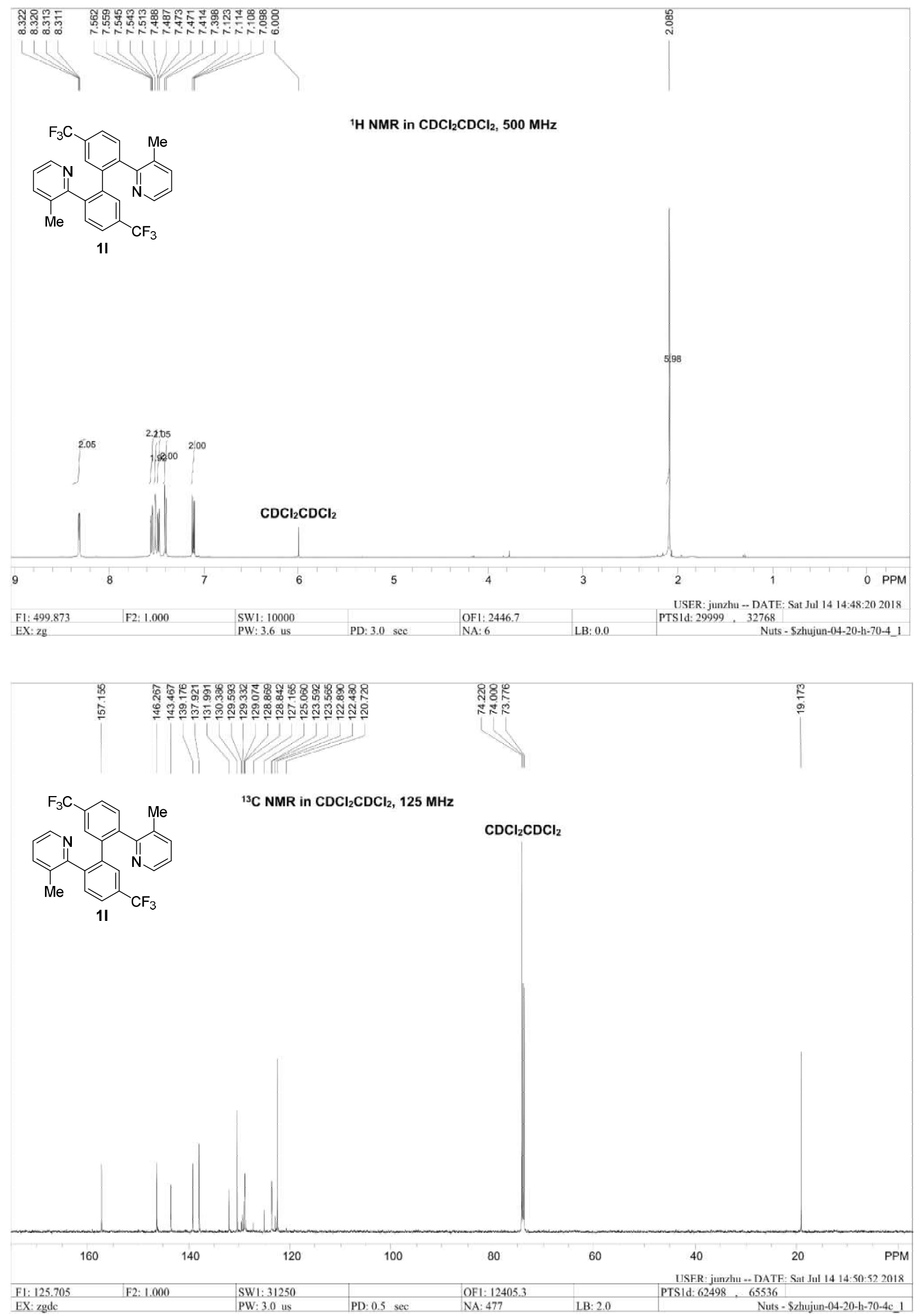


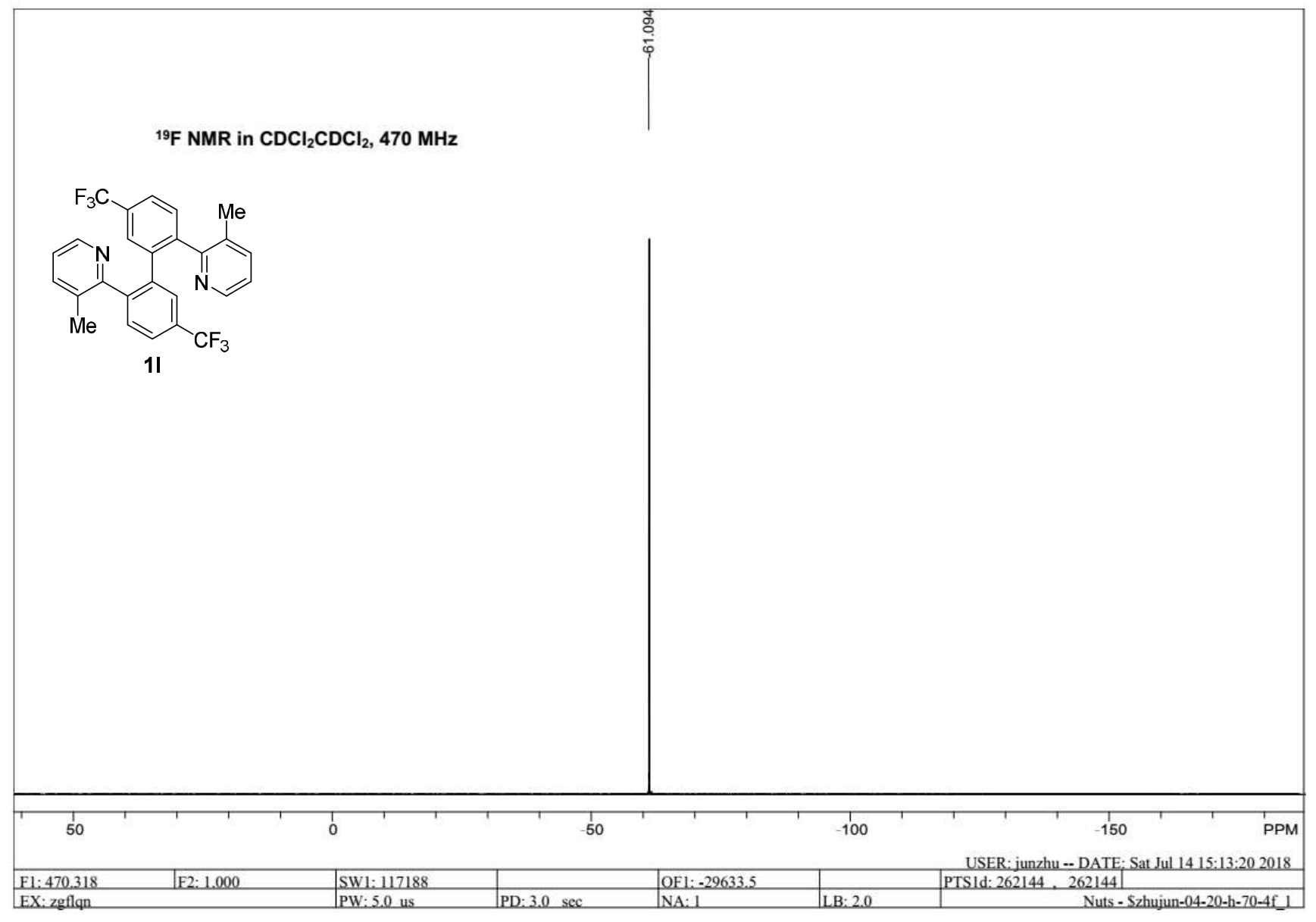



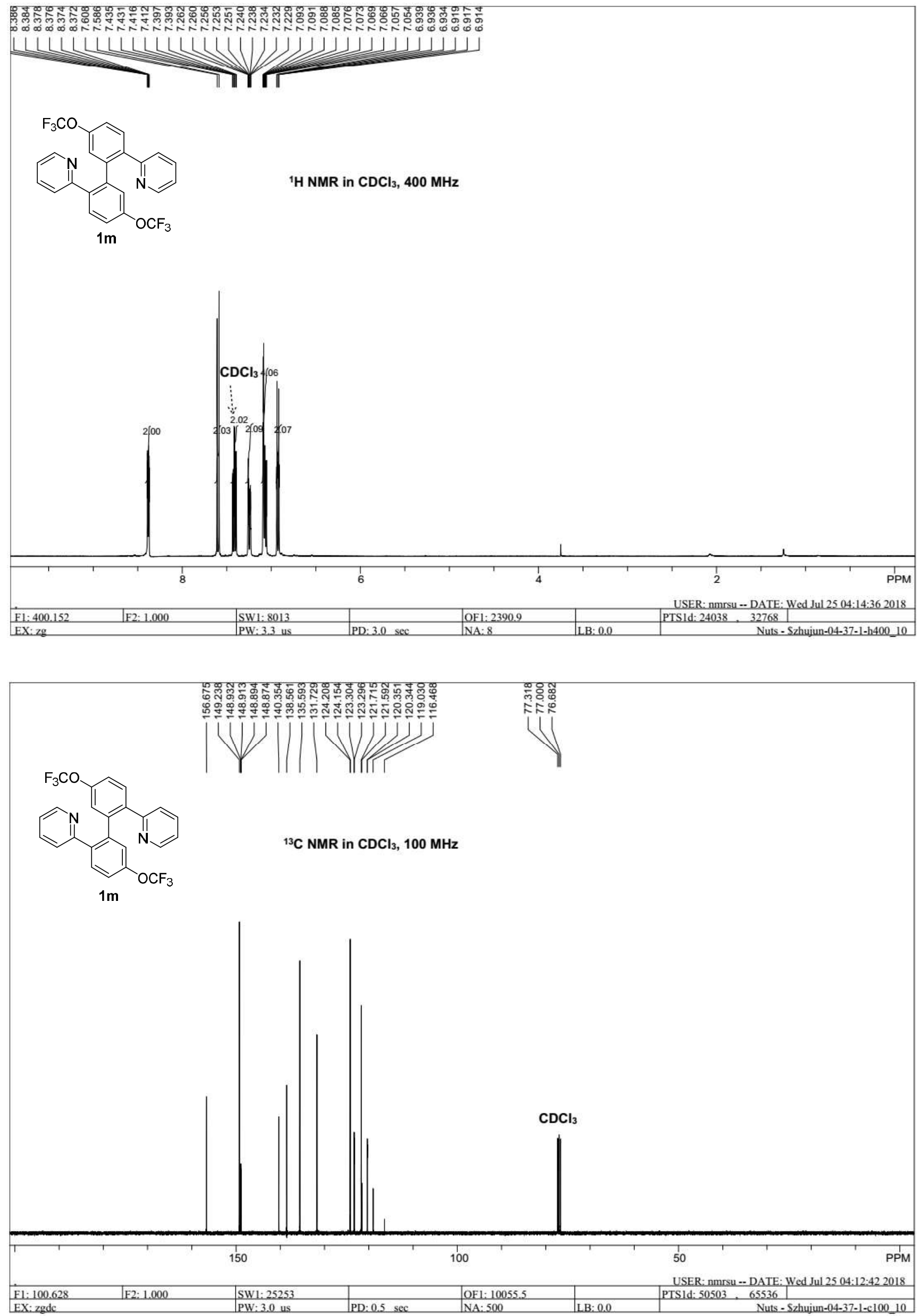


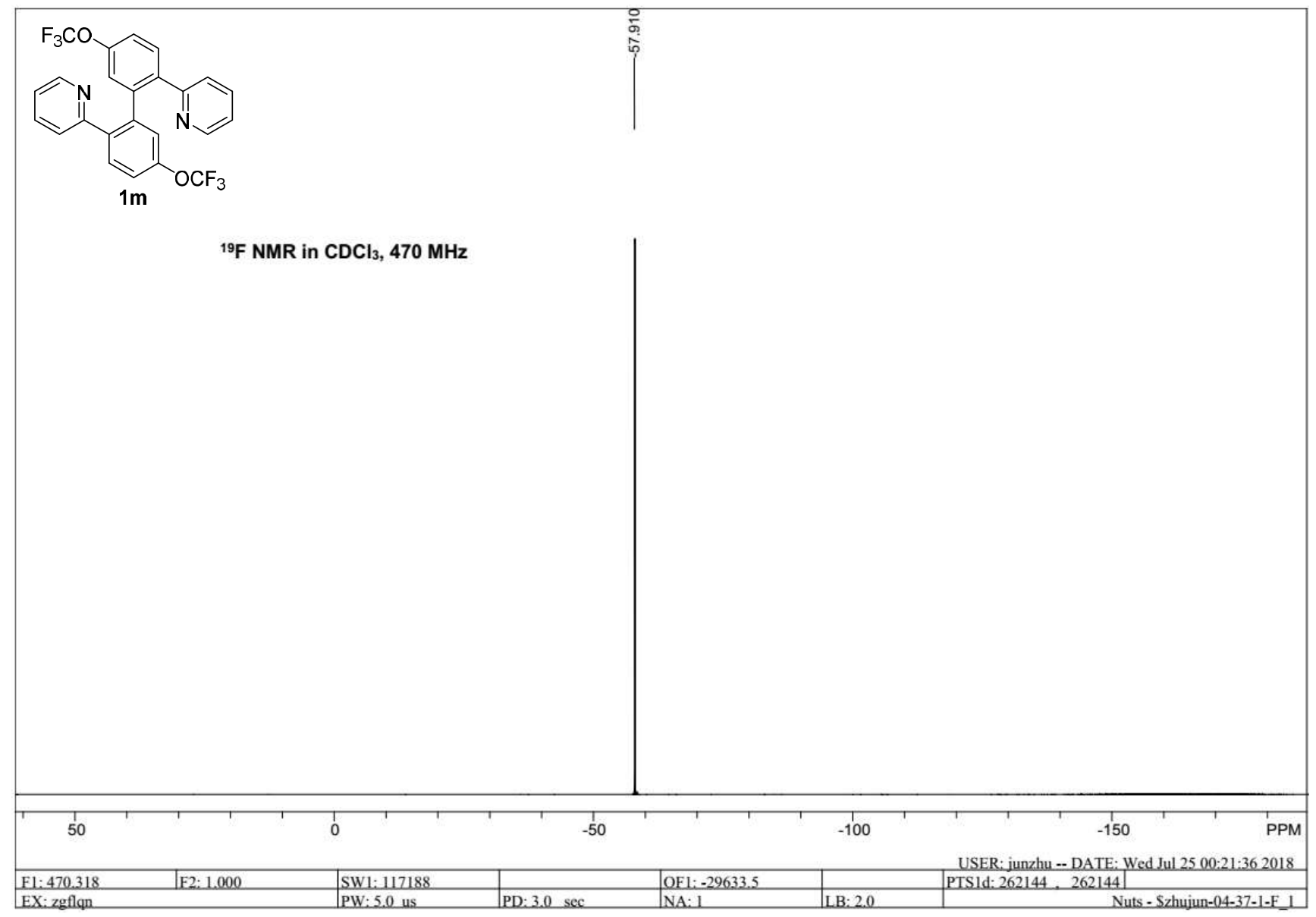



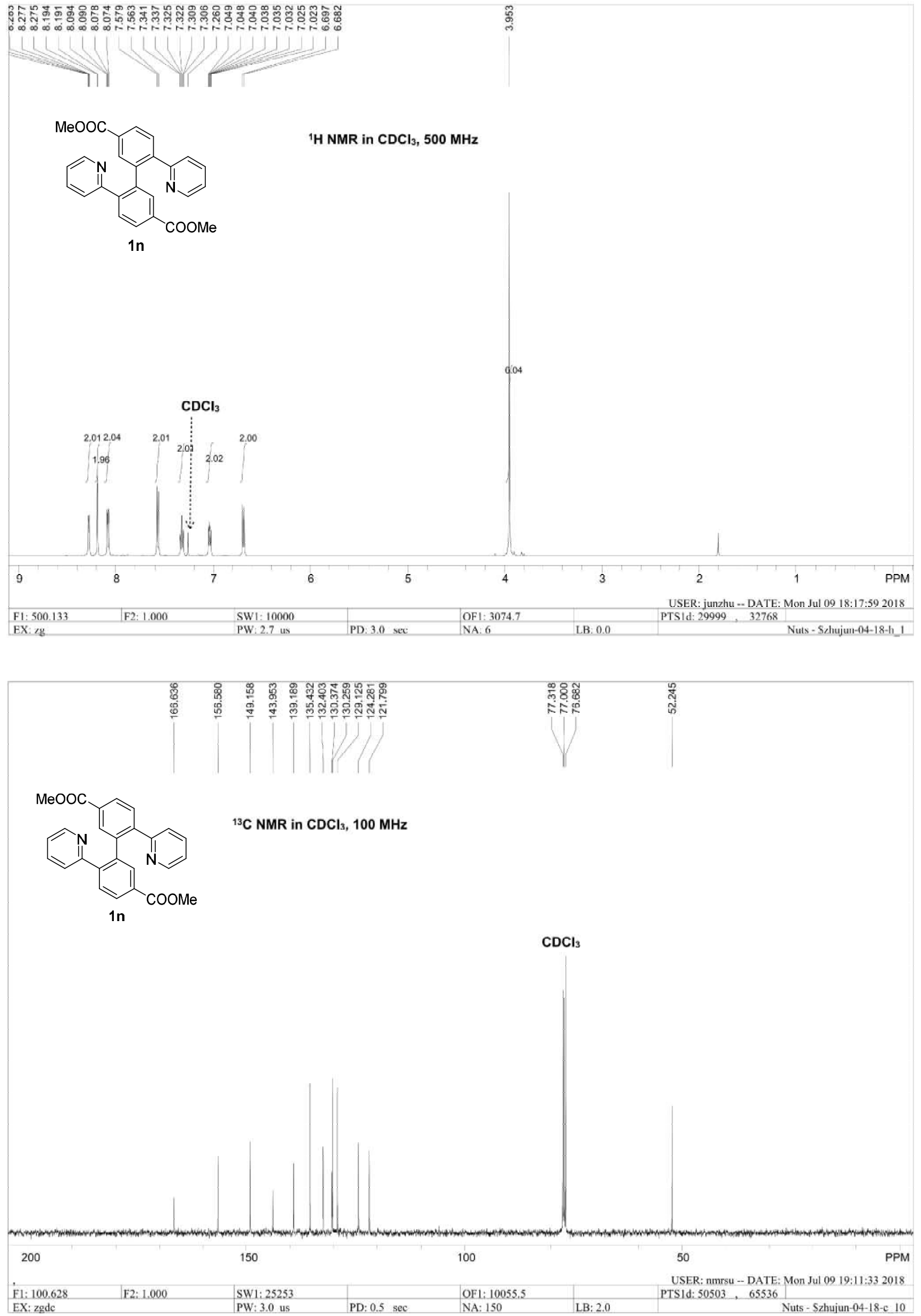


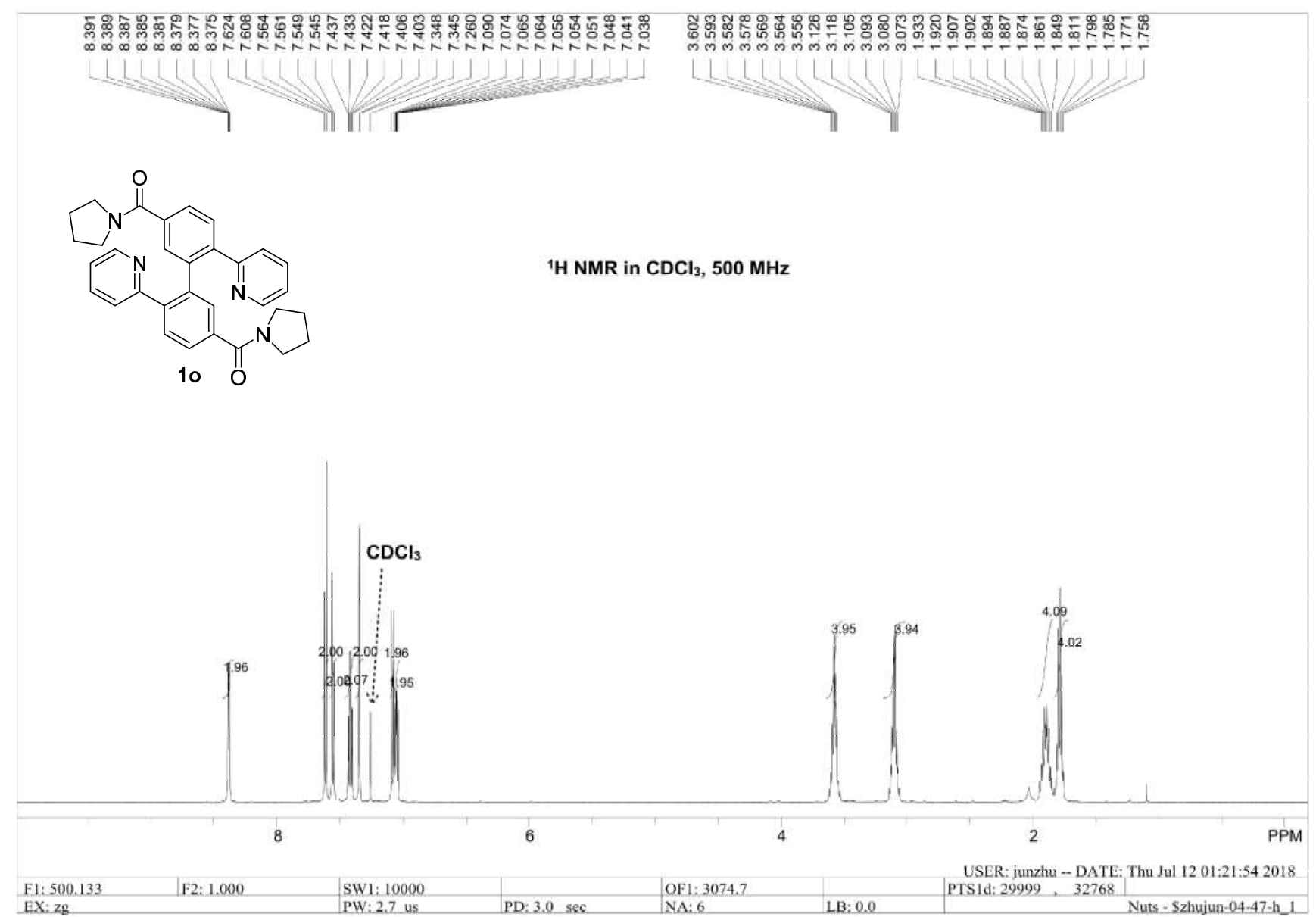

\begin{tabular}{|c|c|c|c|}
\hline & F2: 1.00 & SW1: 10000 & PTS1d: $29999, \quad 32768$ \\
\hline
\end{tabular}

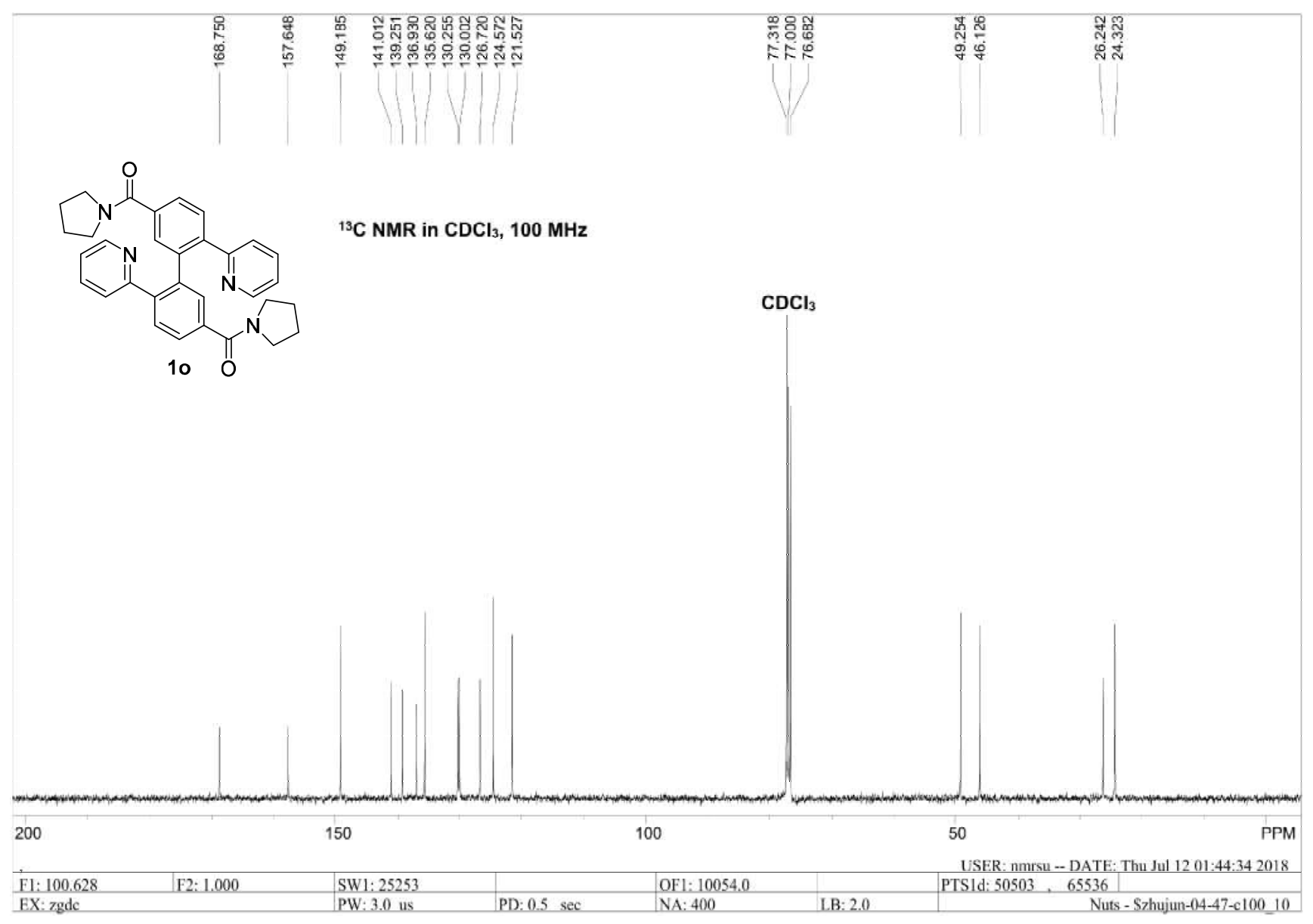



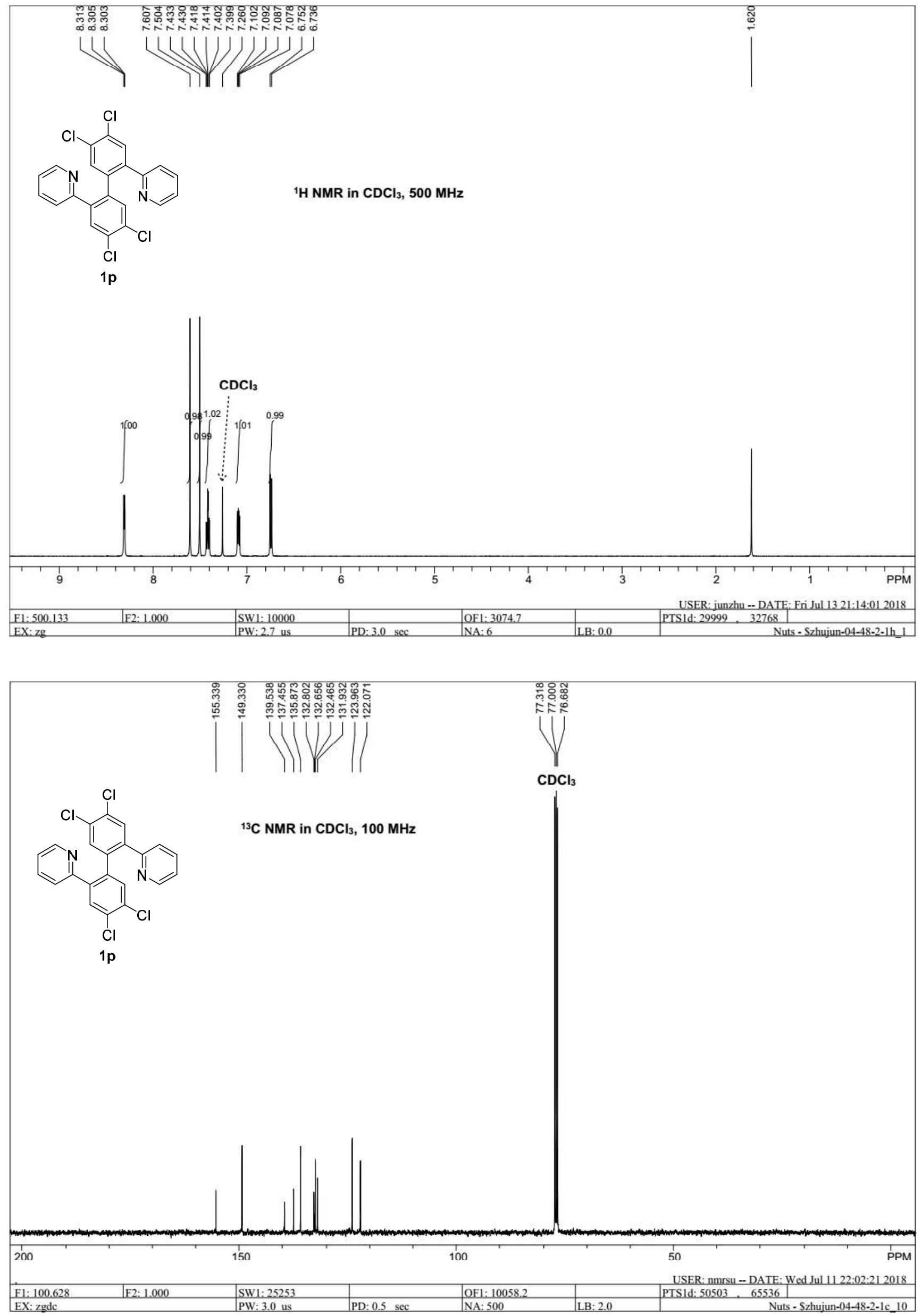

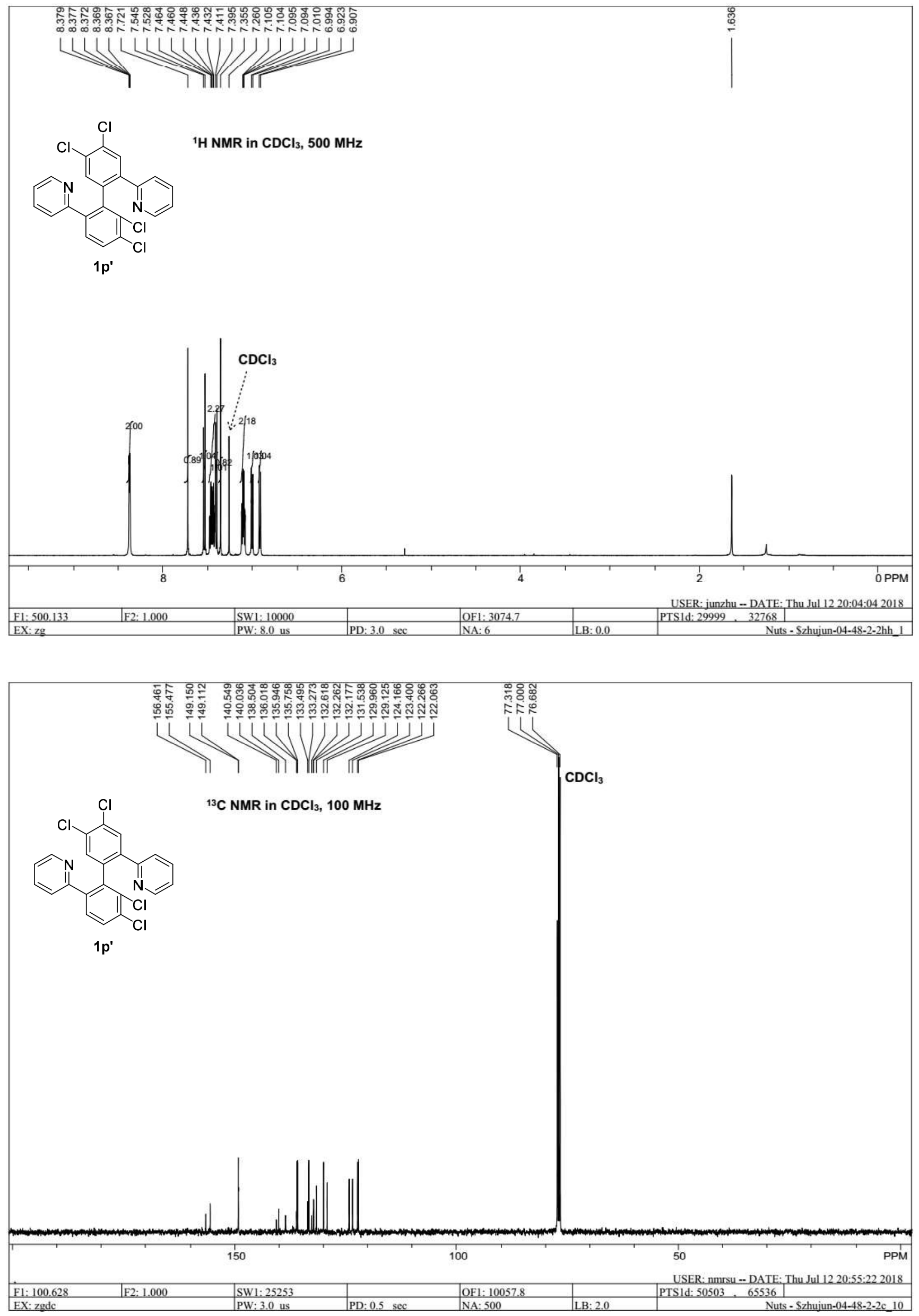

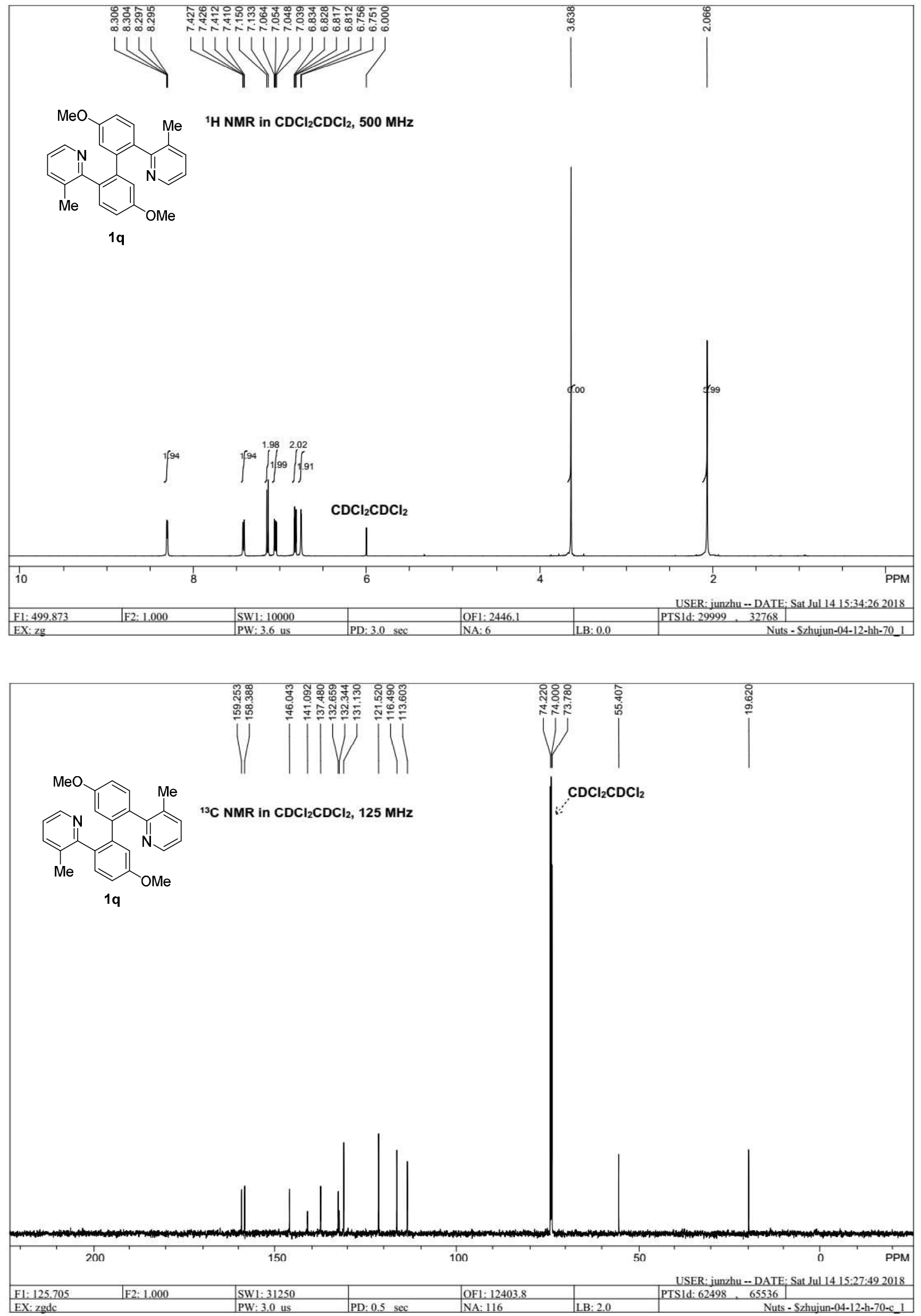

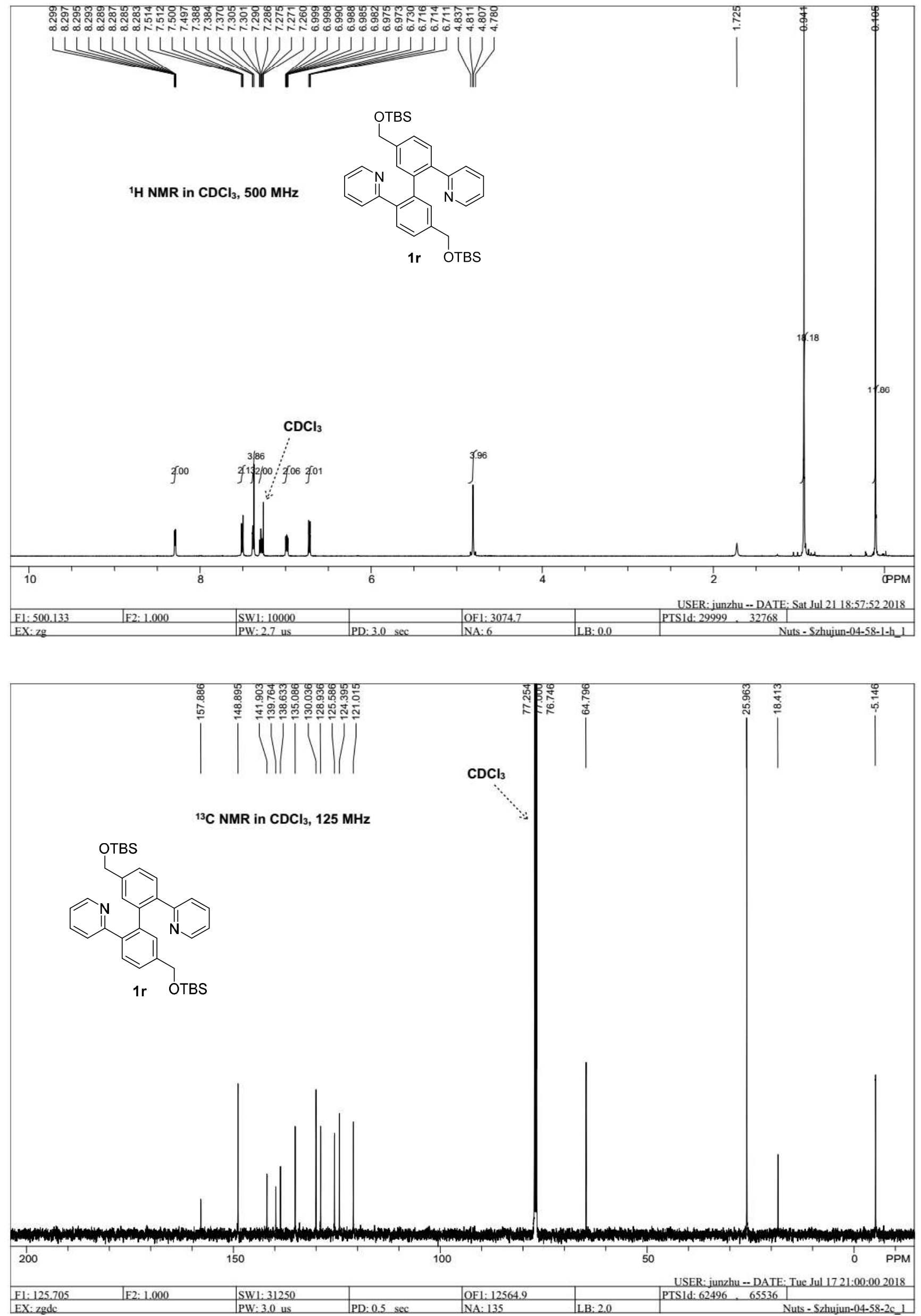

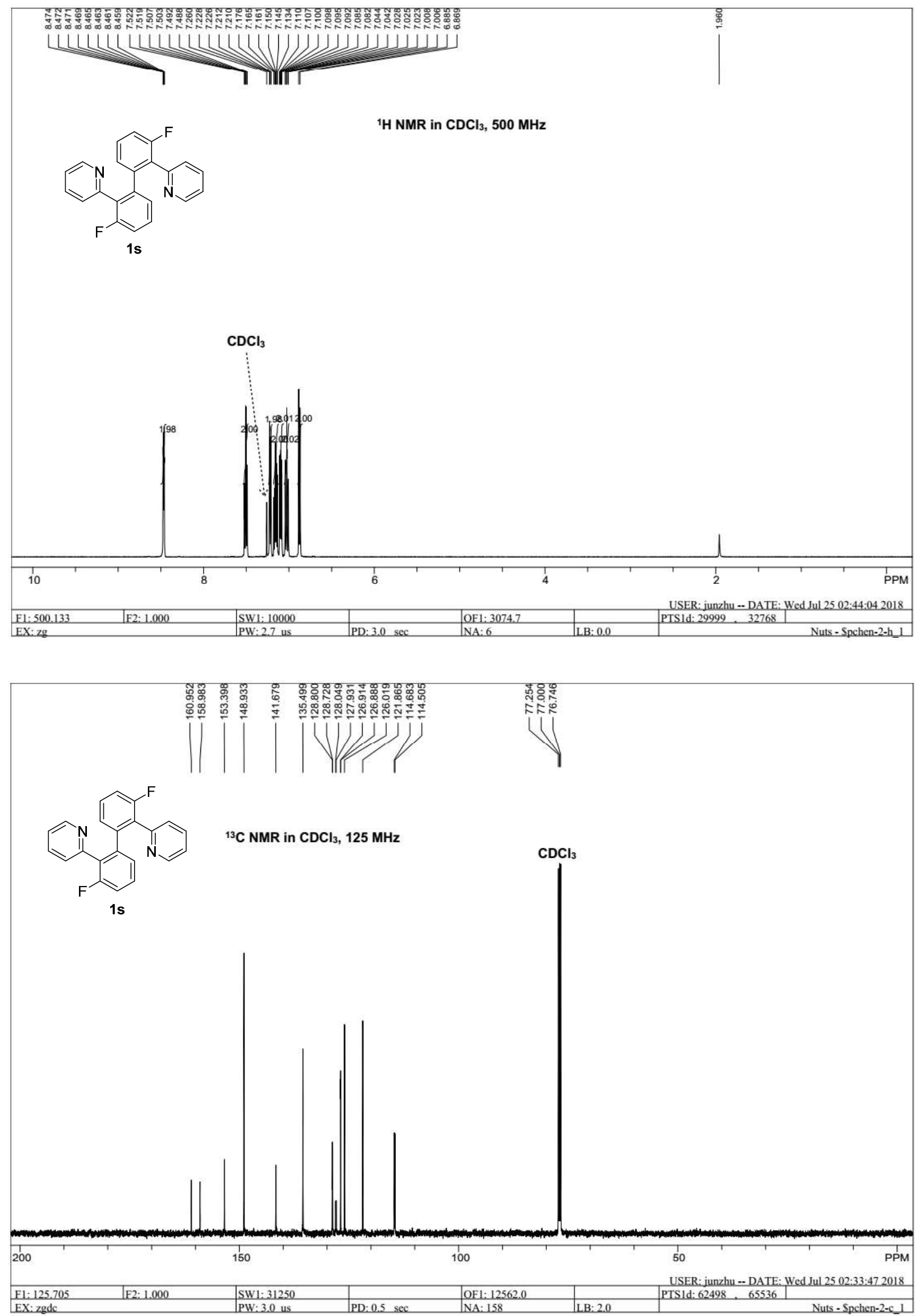


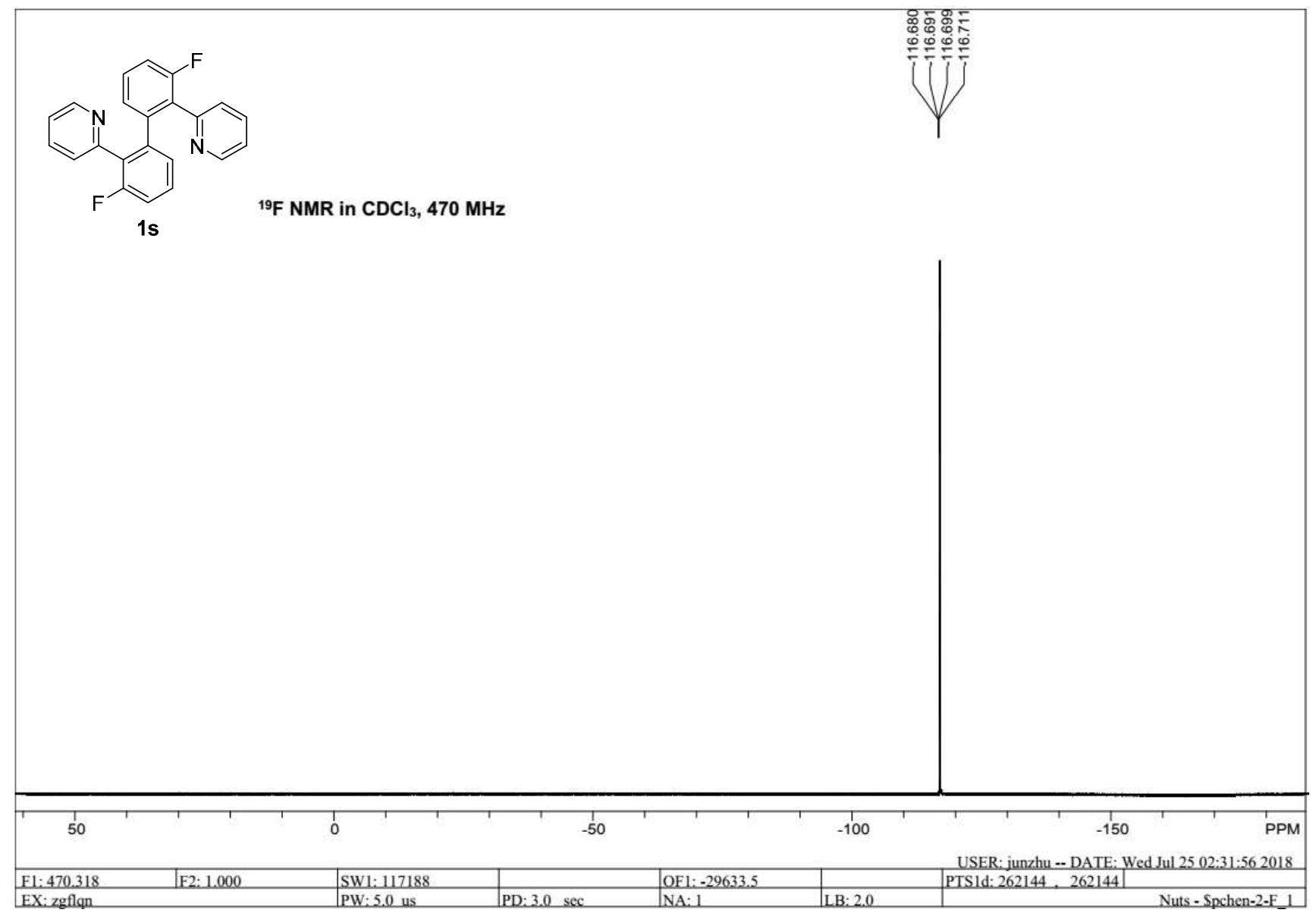



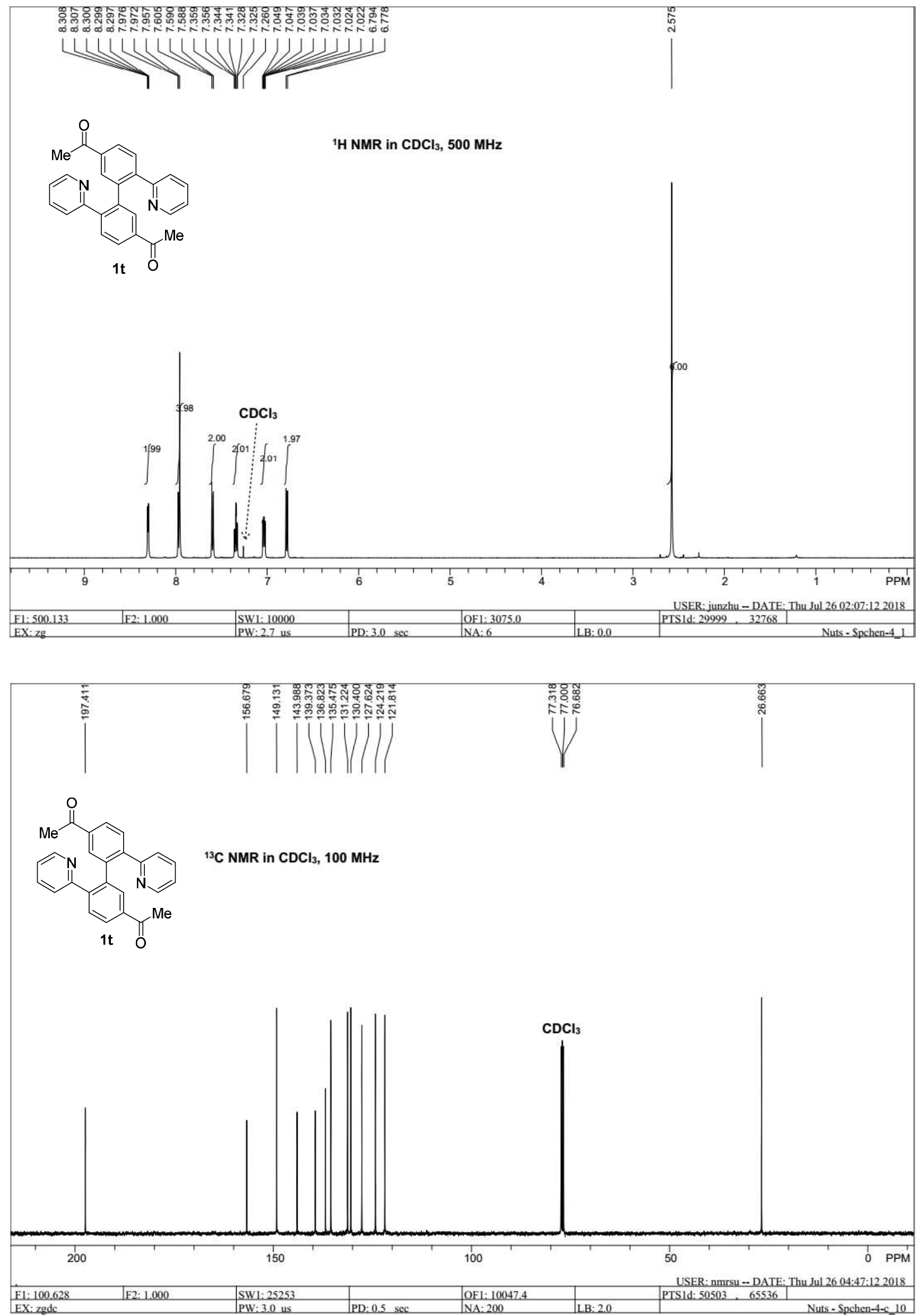

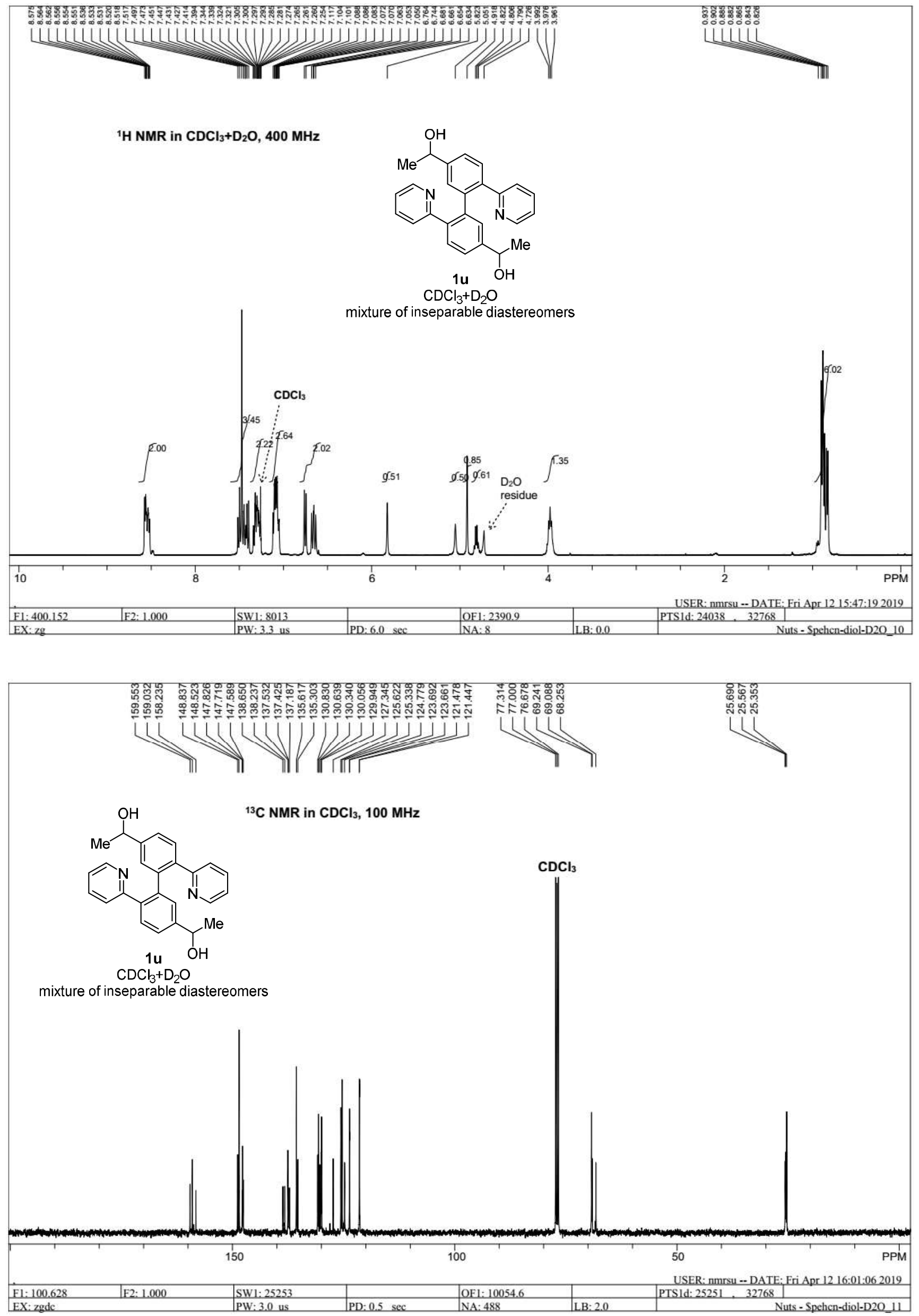

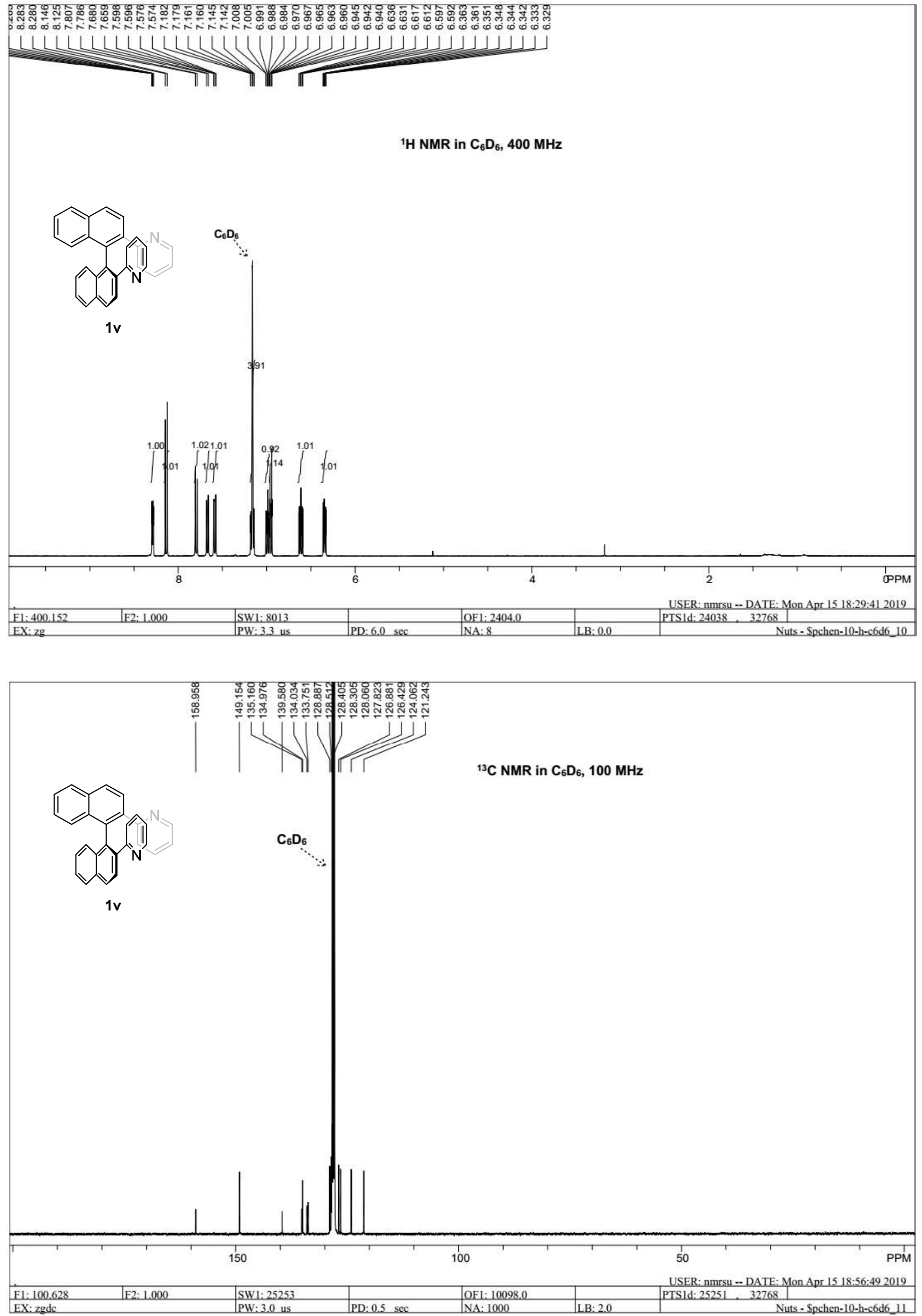

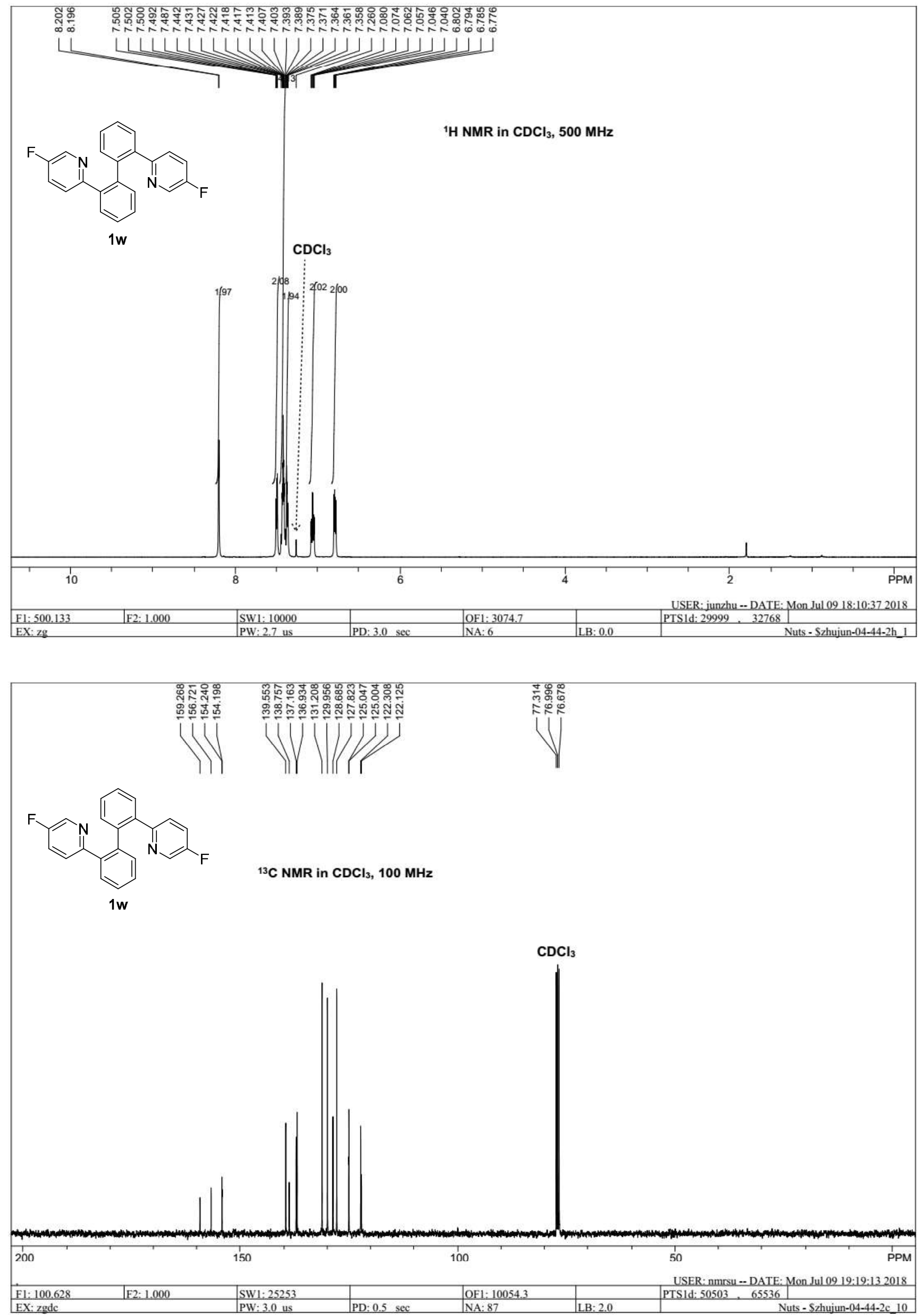


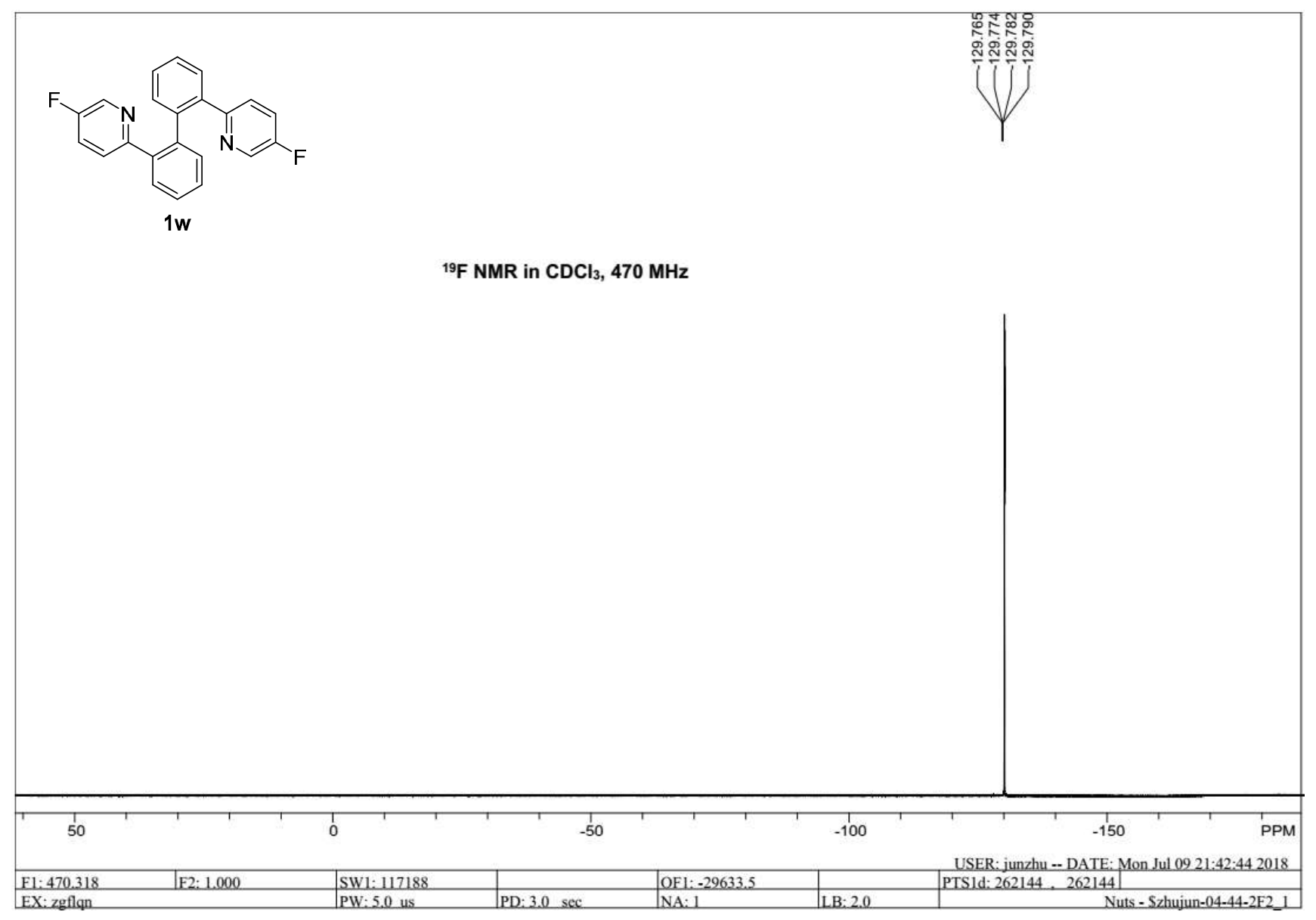



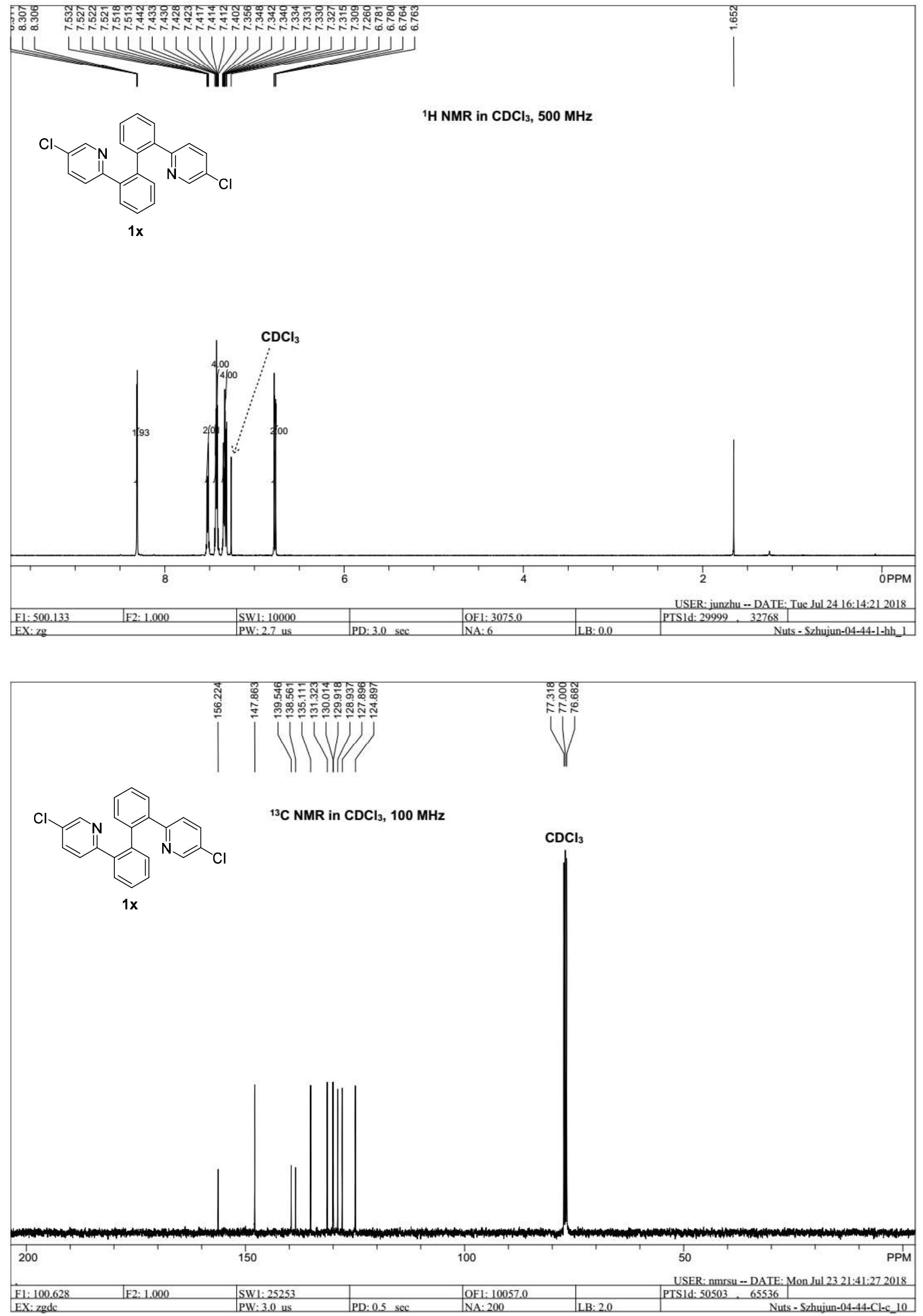

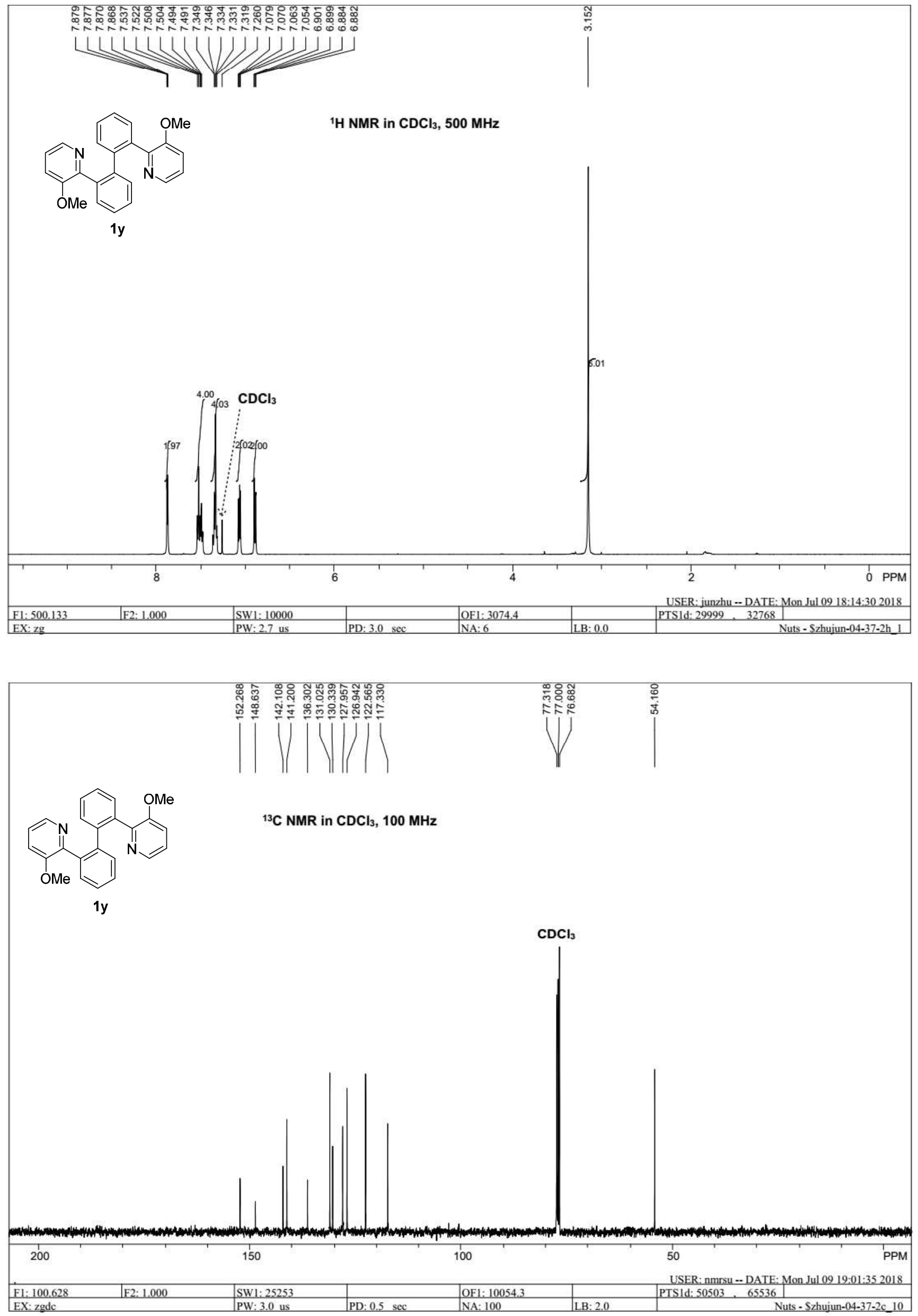

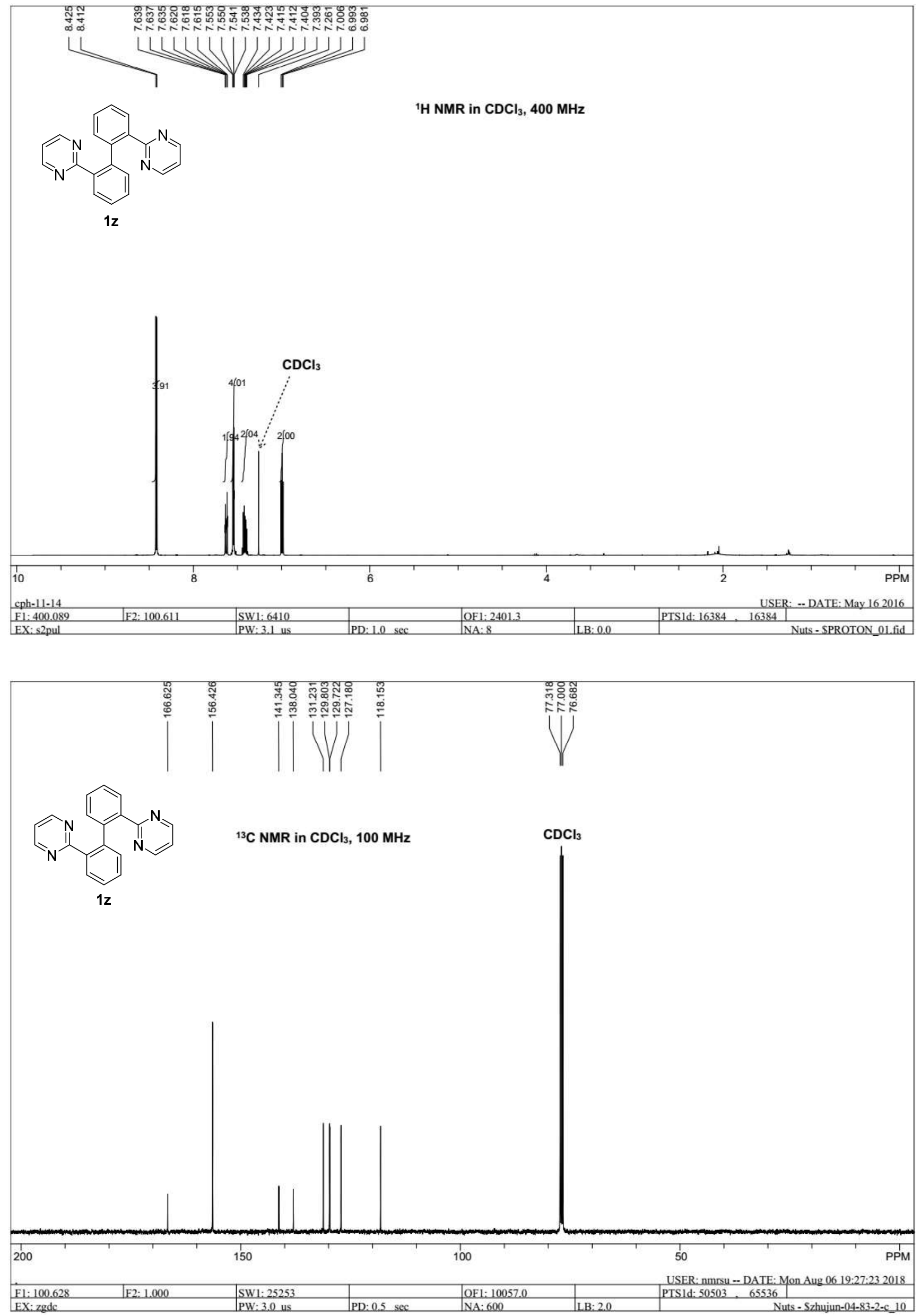

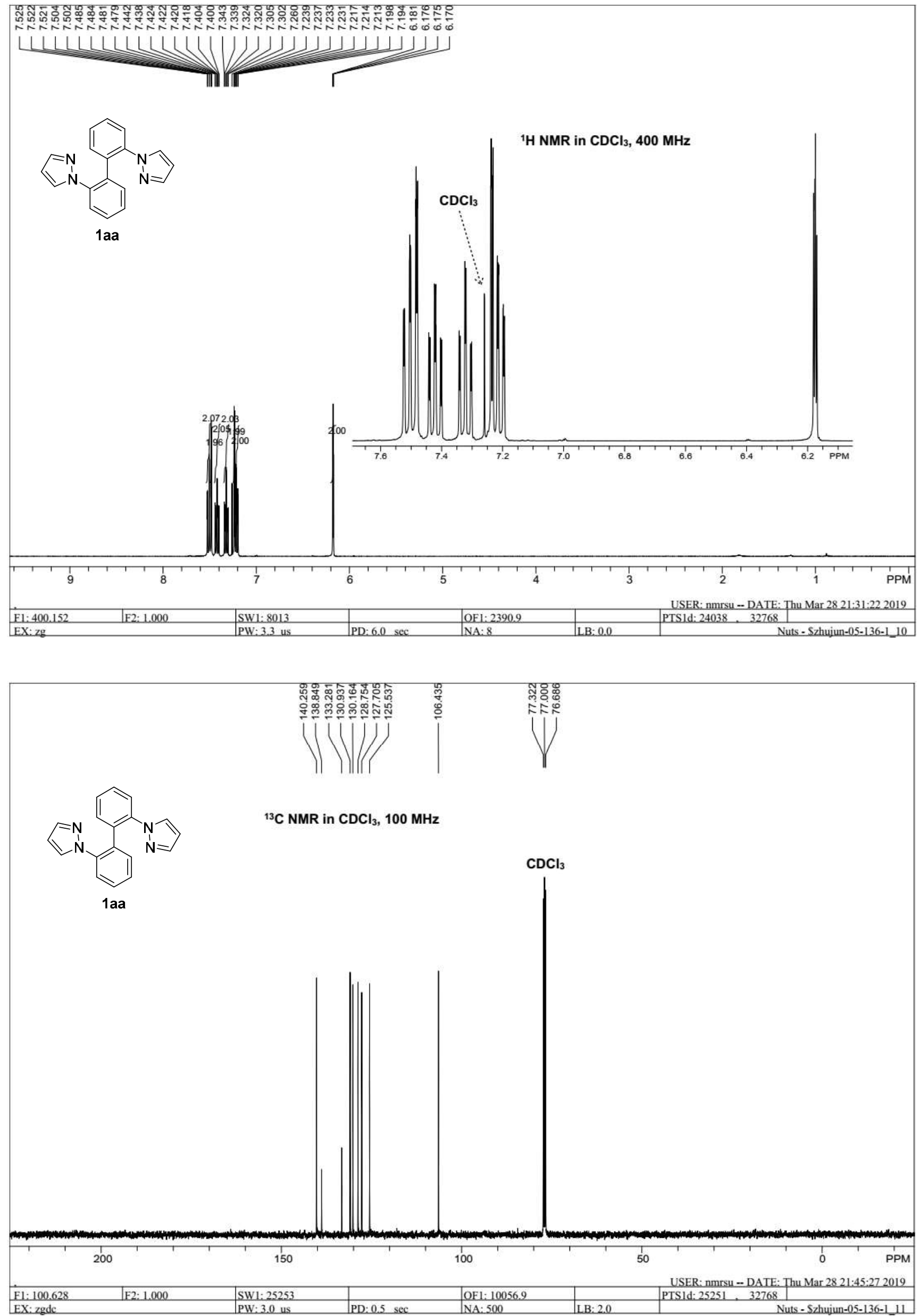

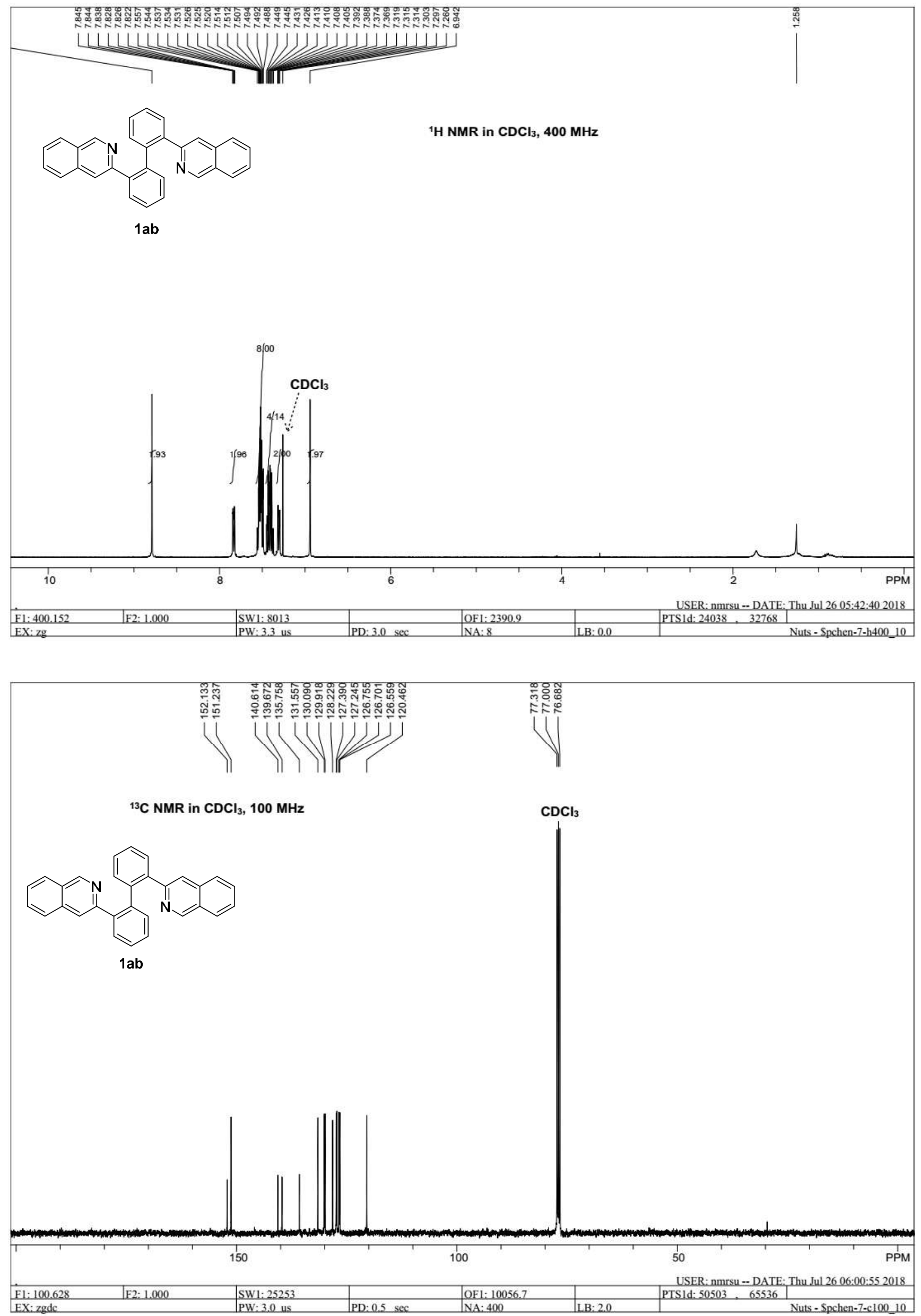

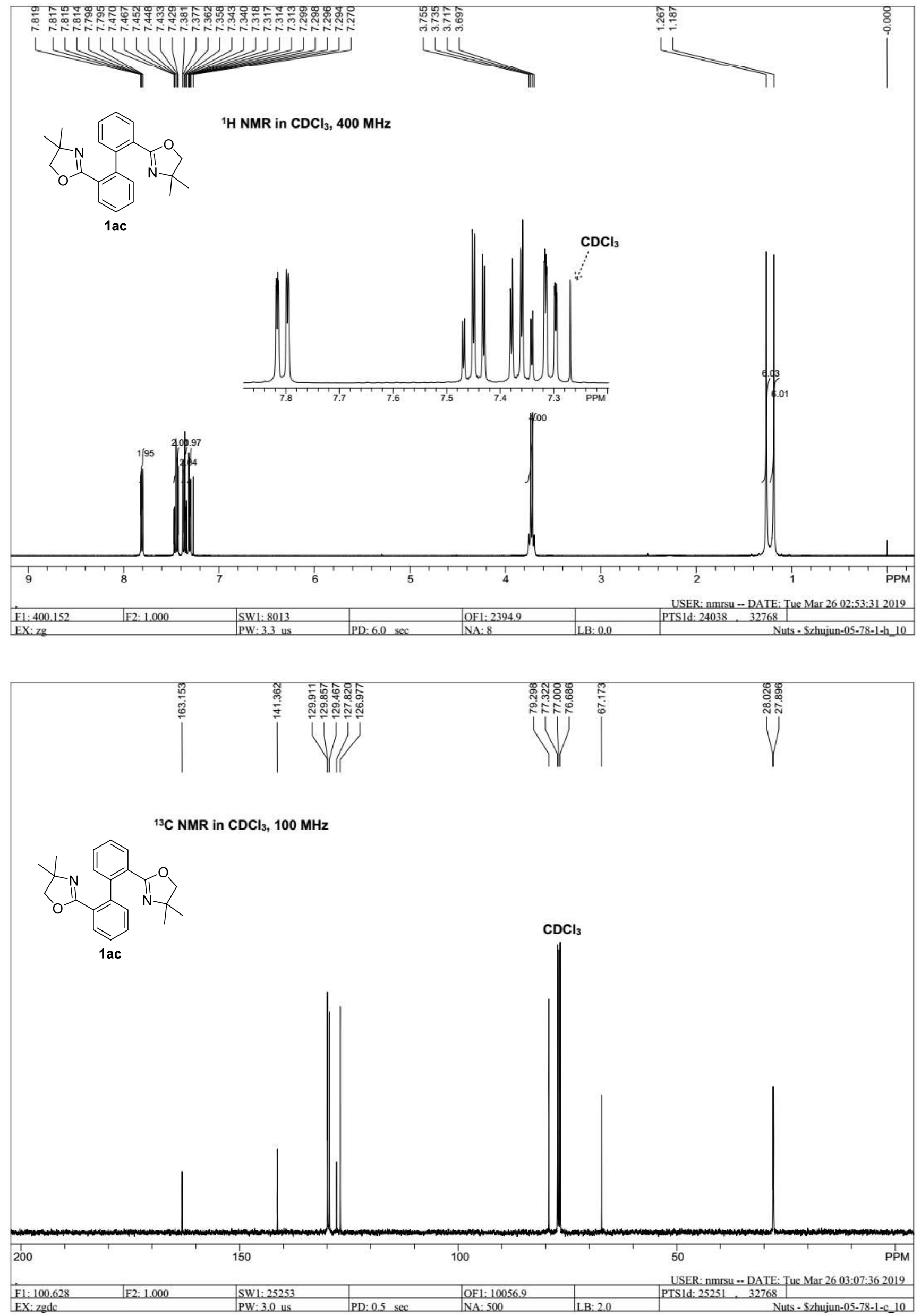

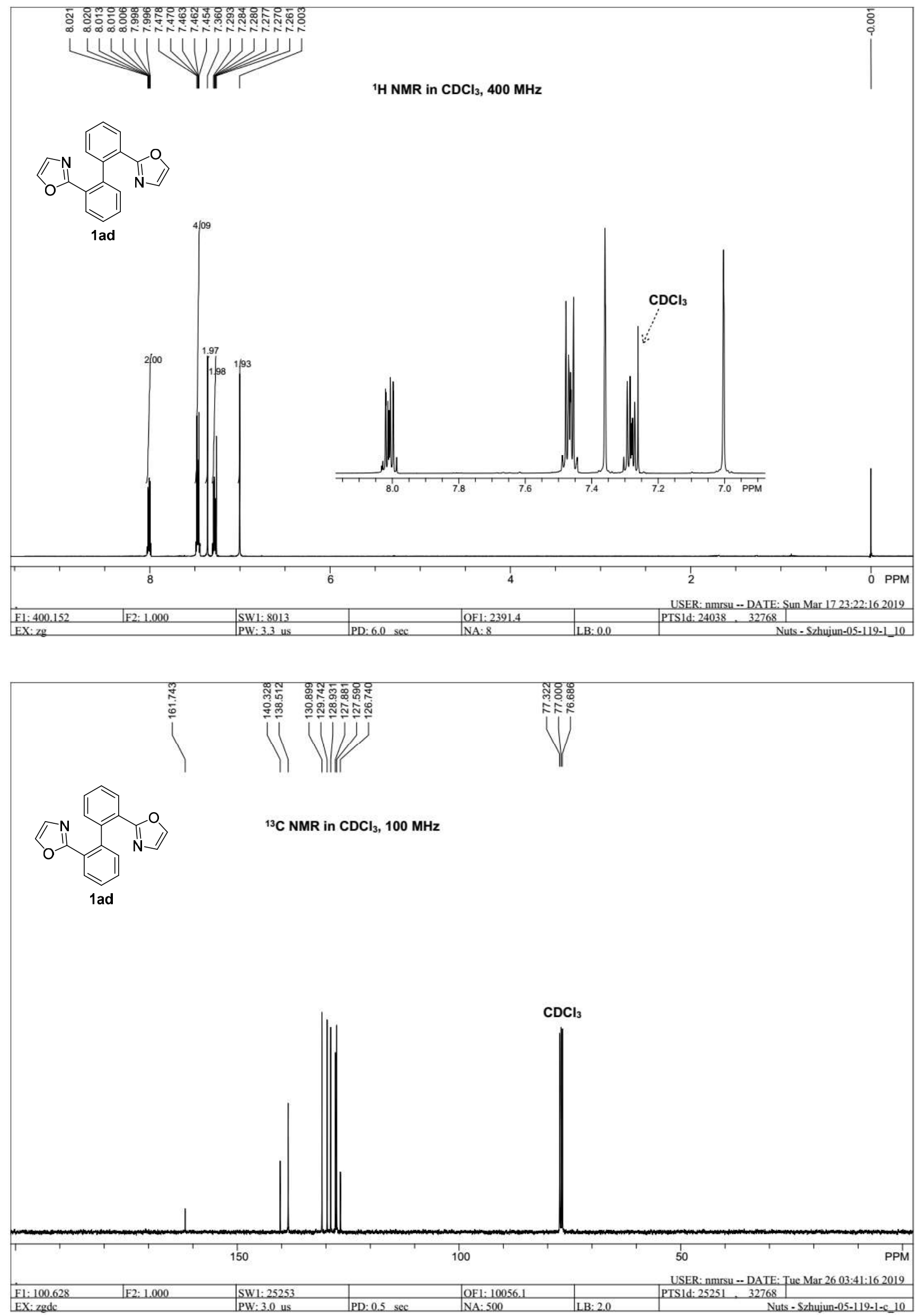

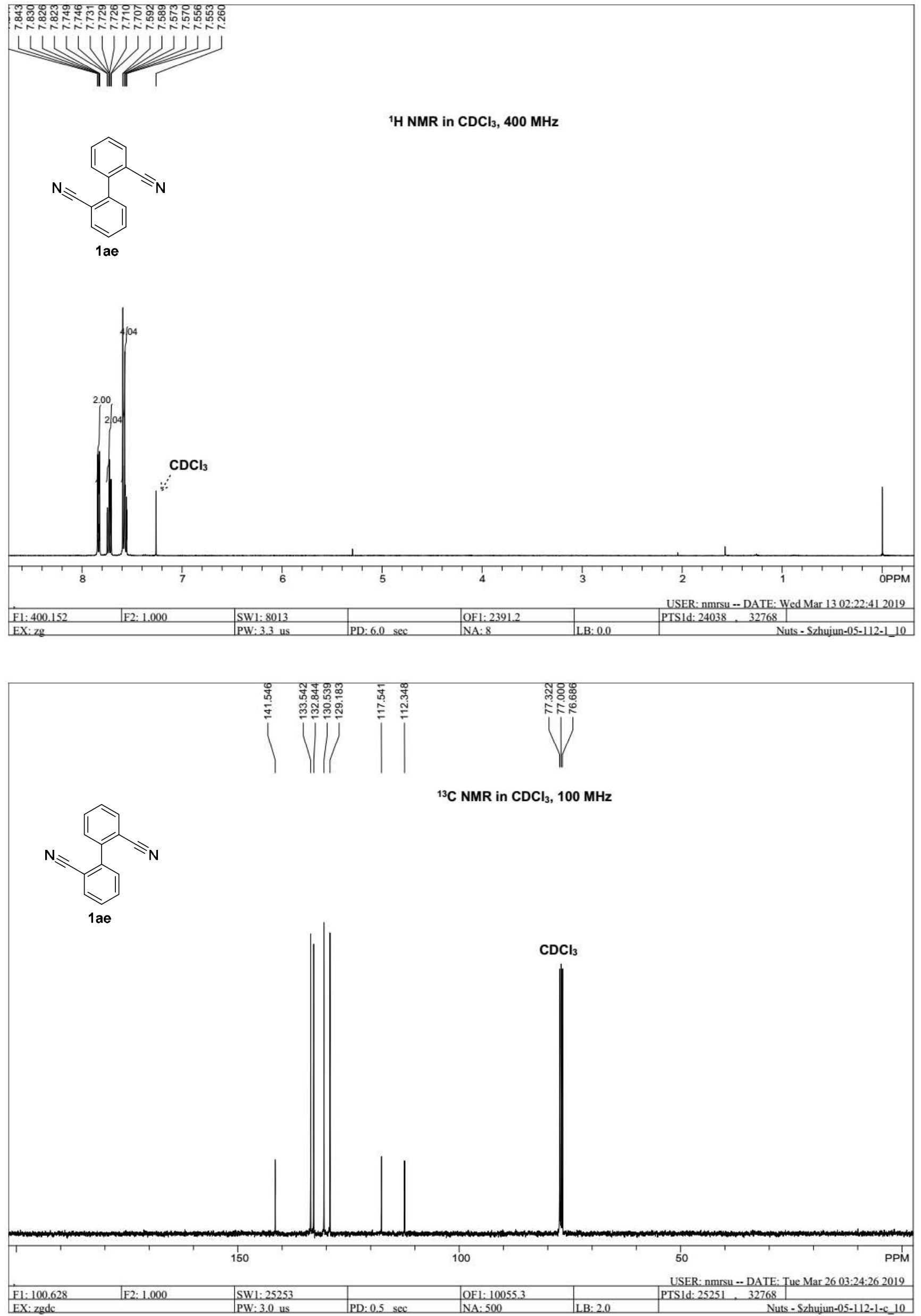

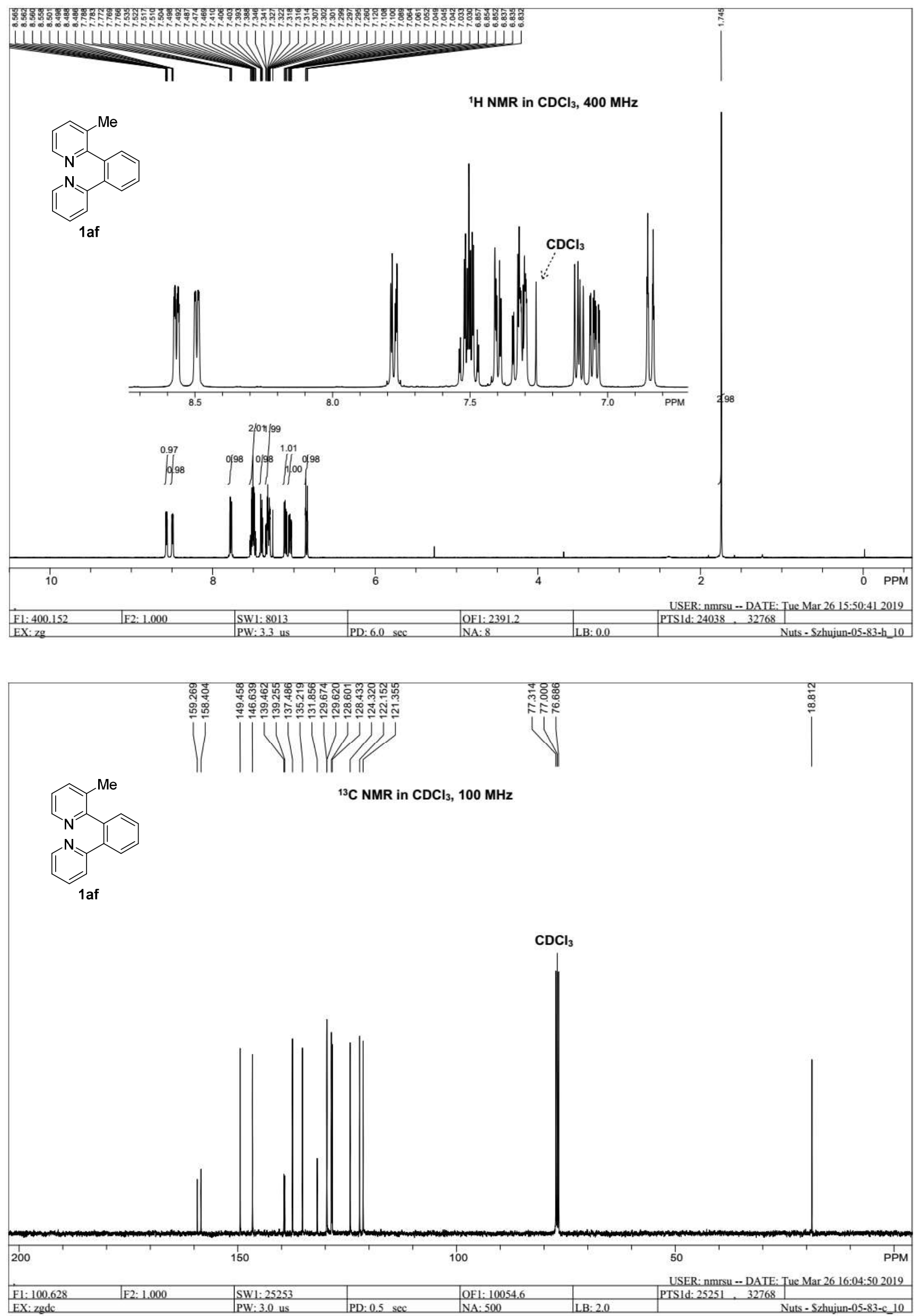


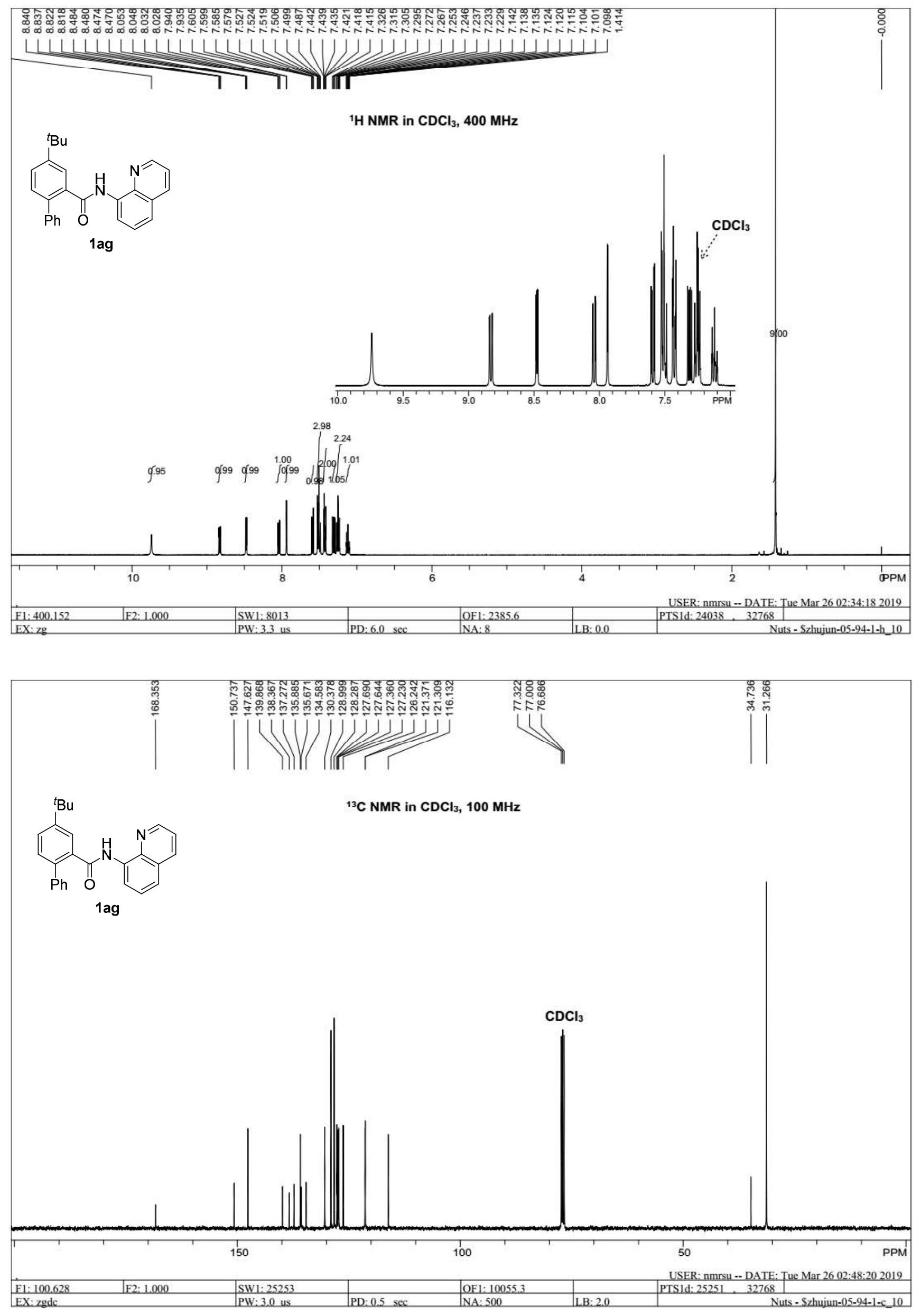



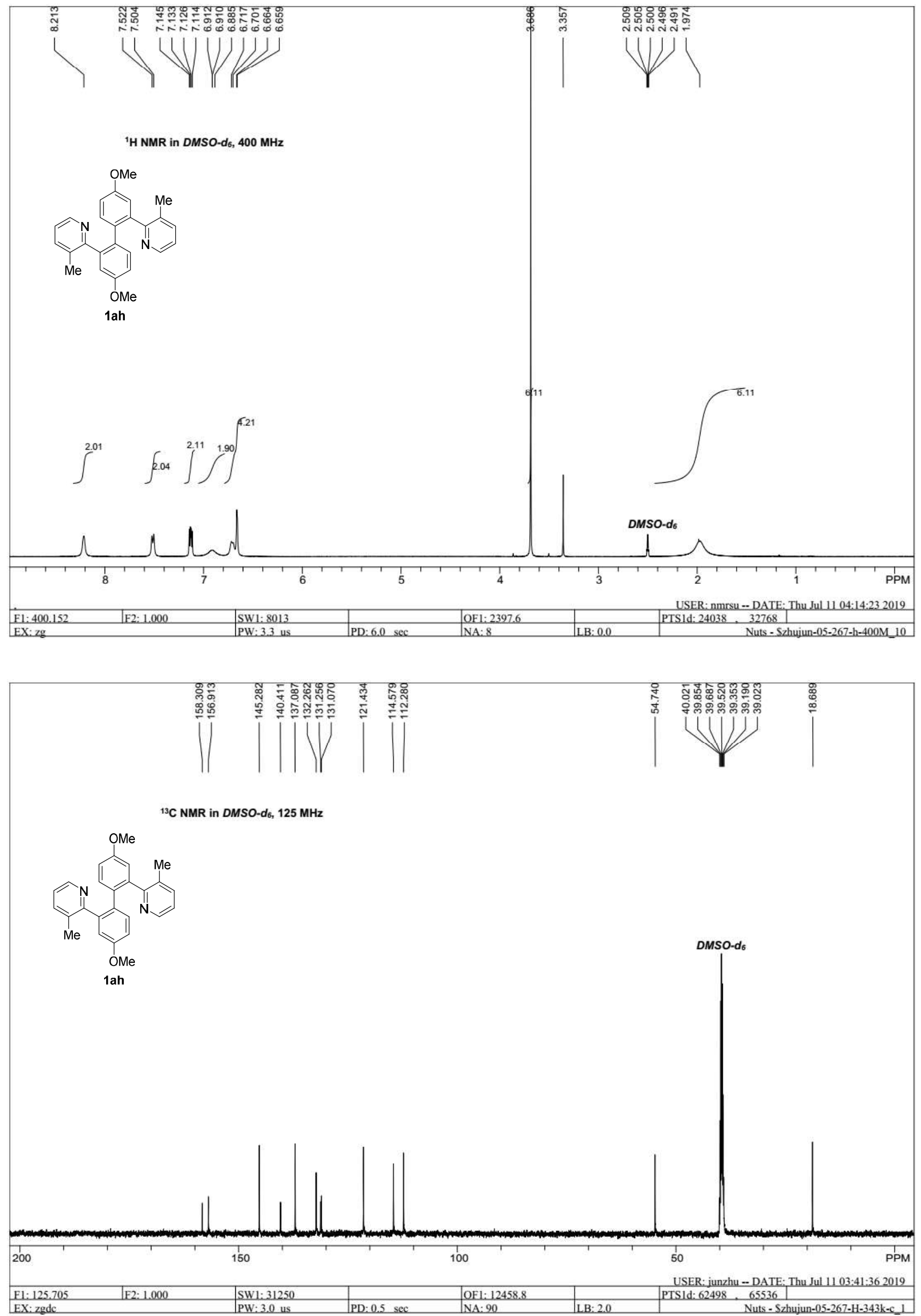

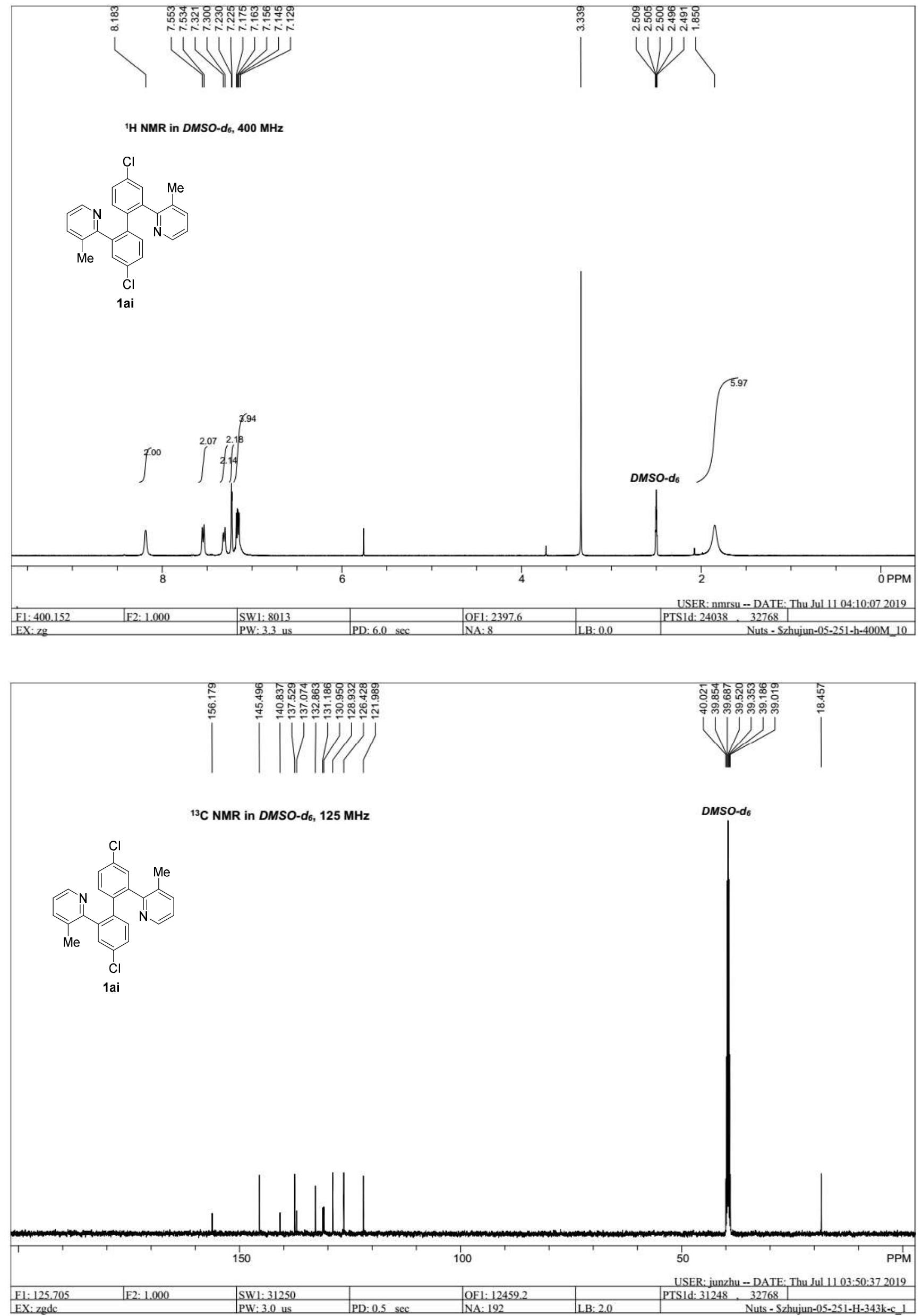

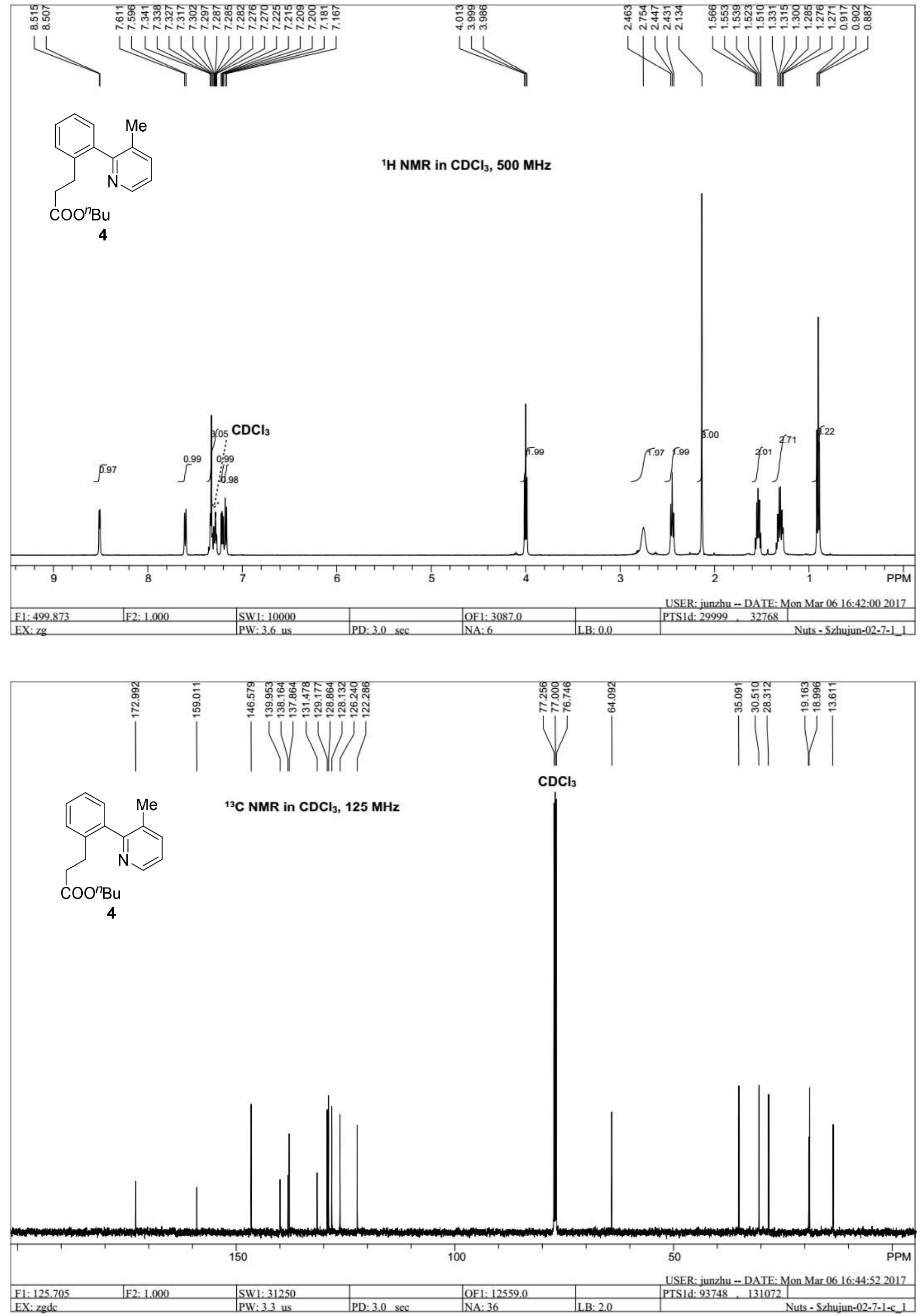

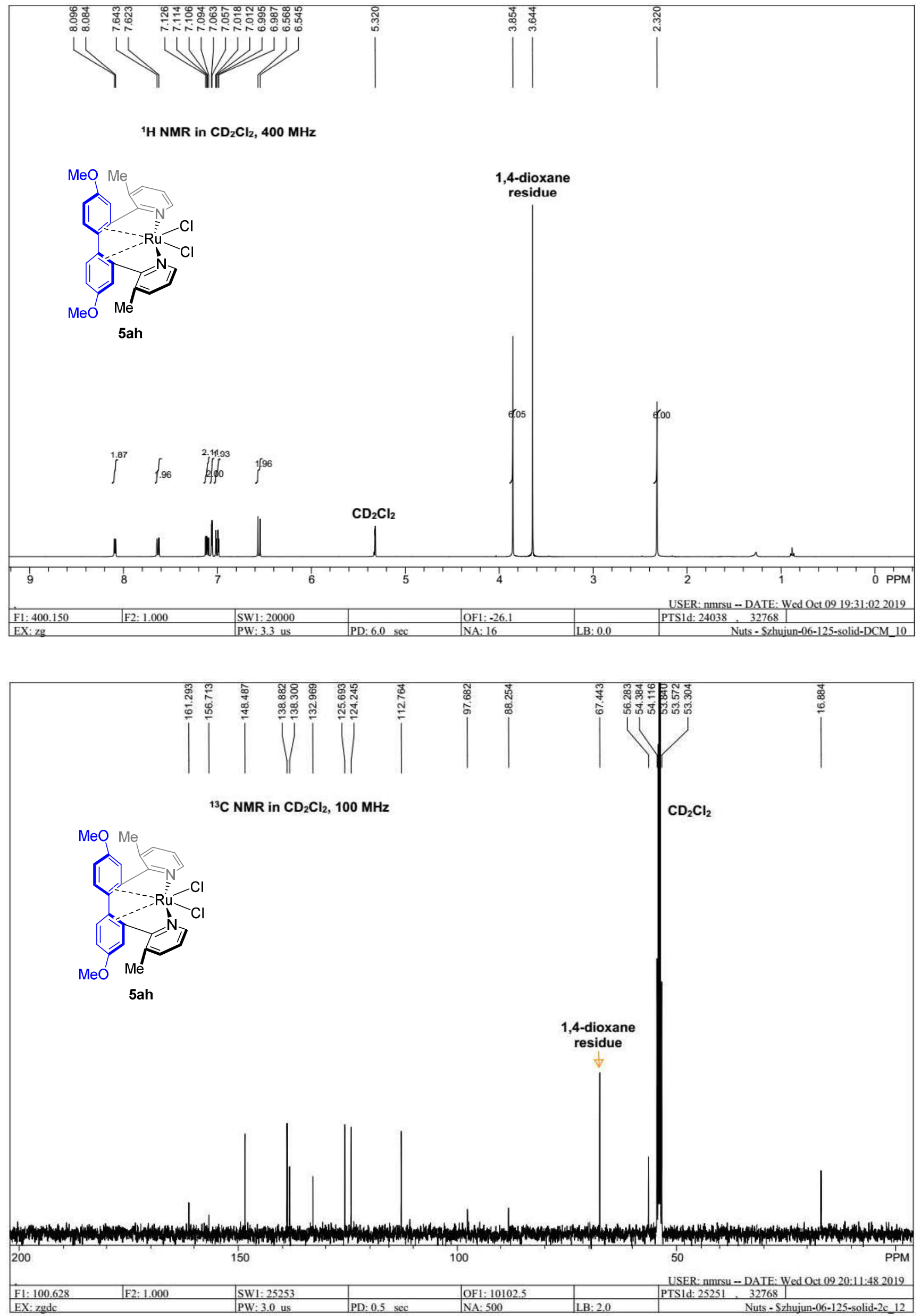

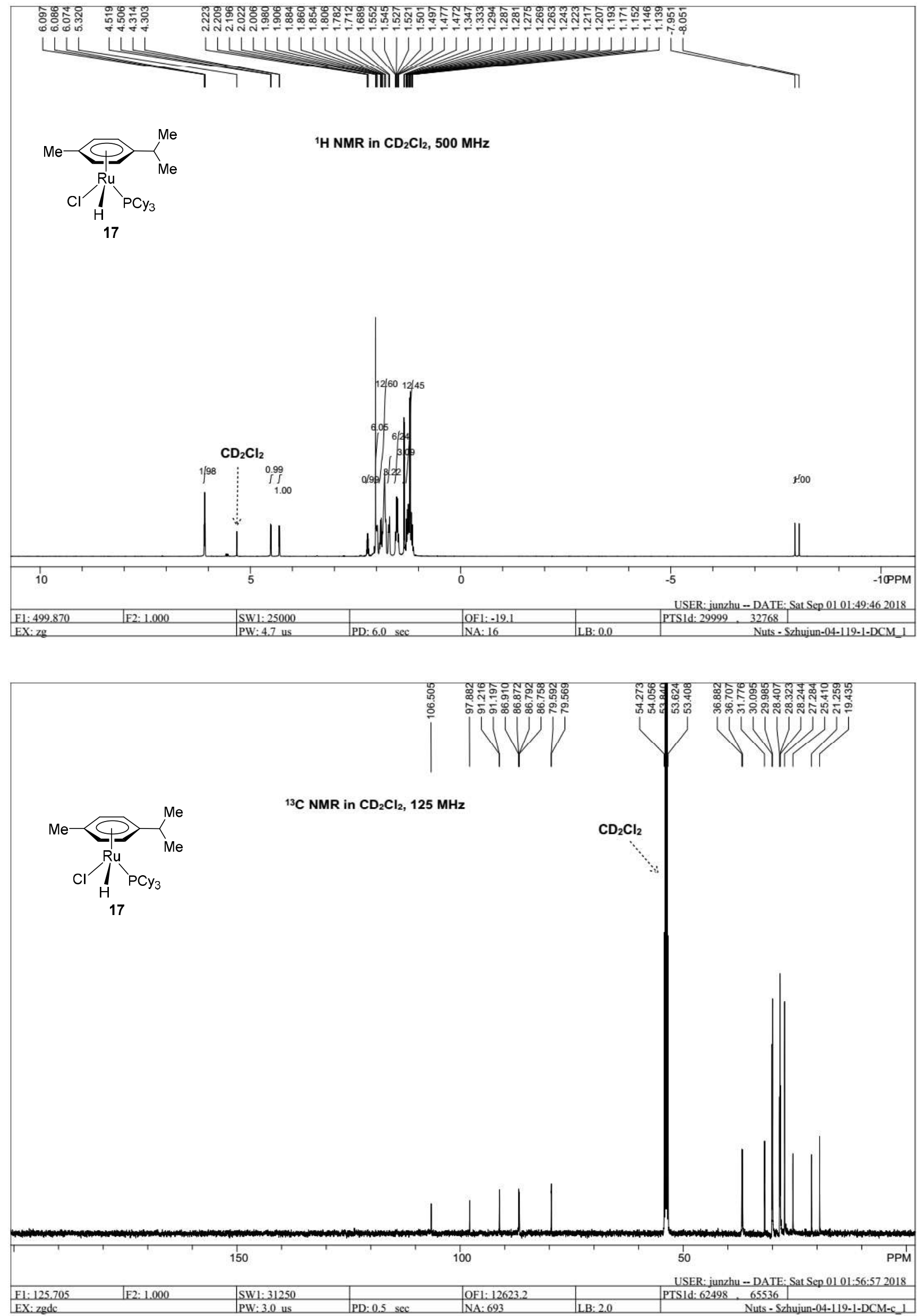

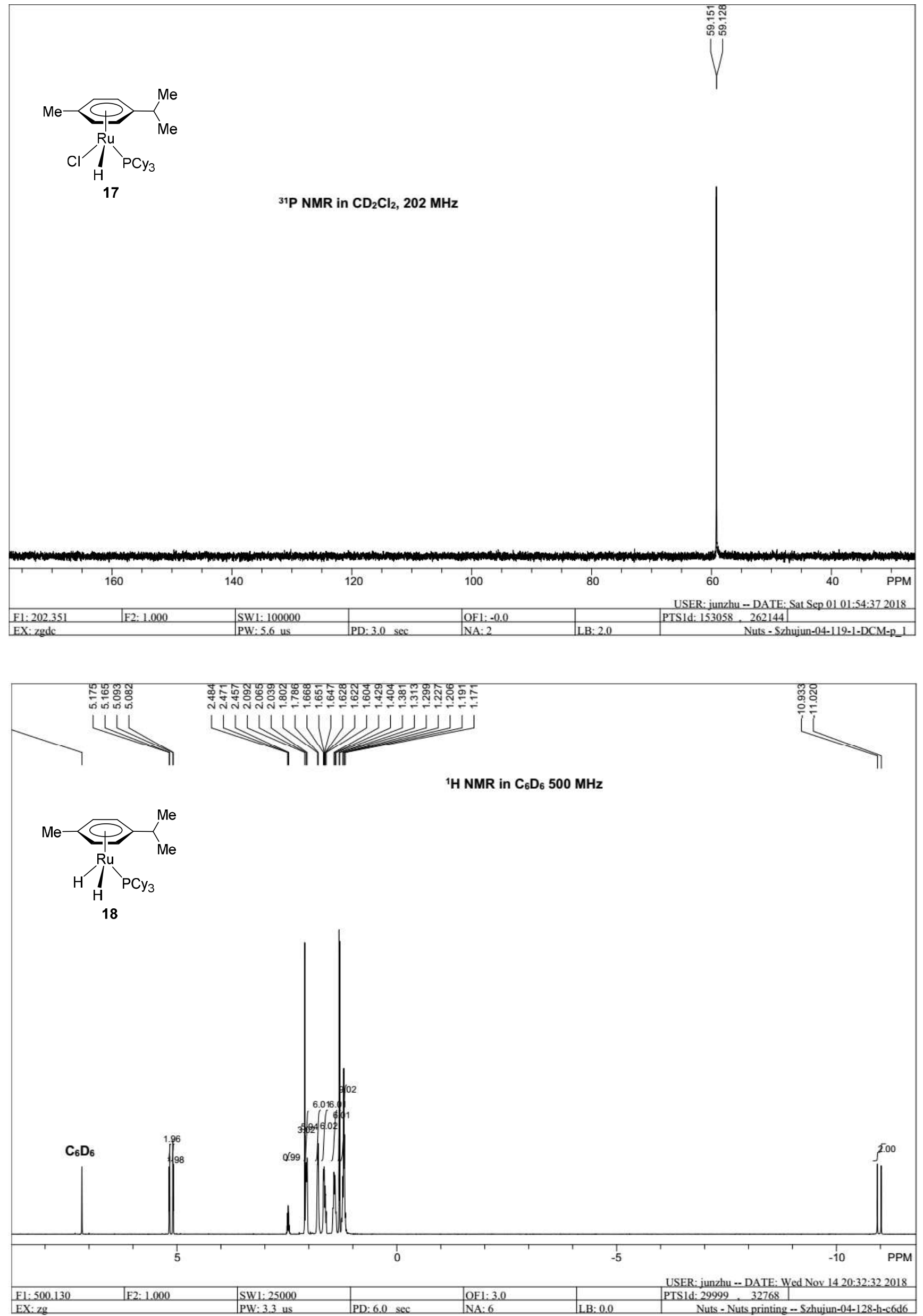

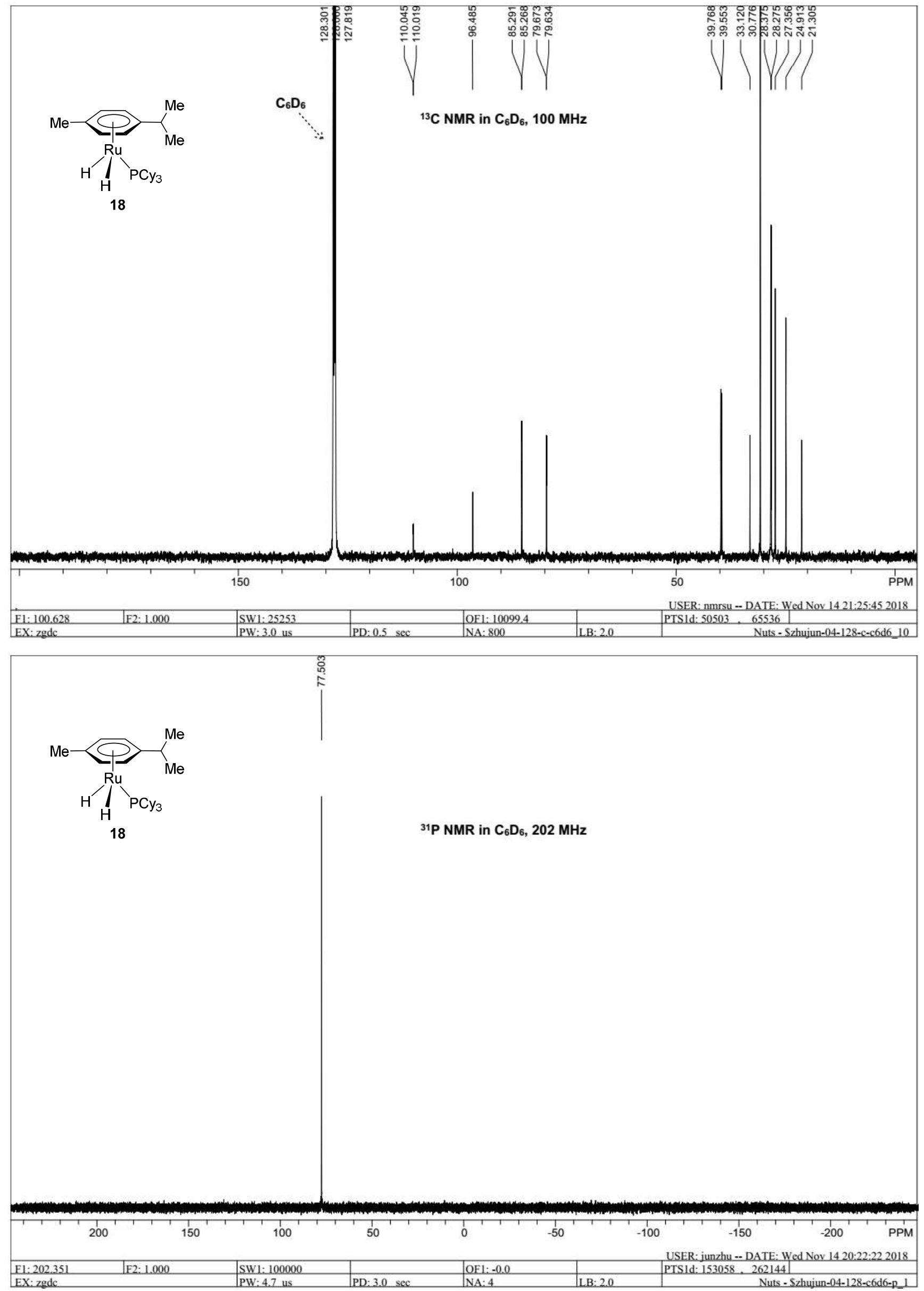

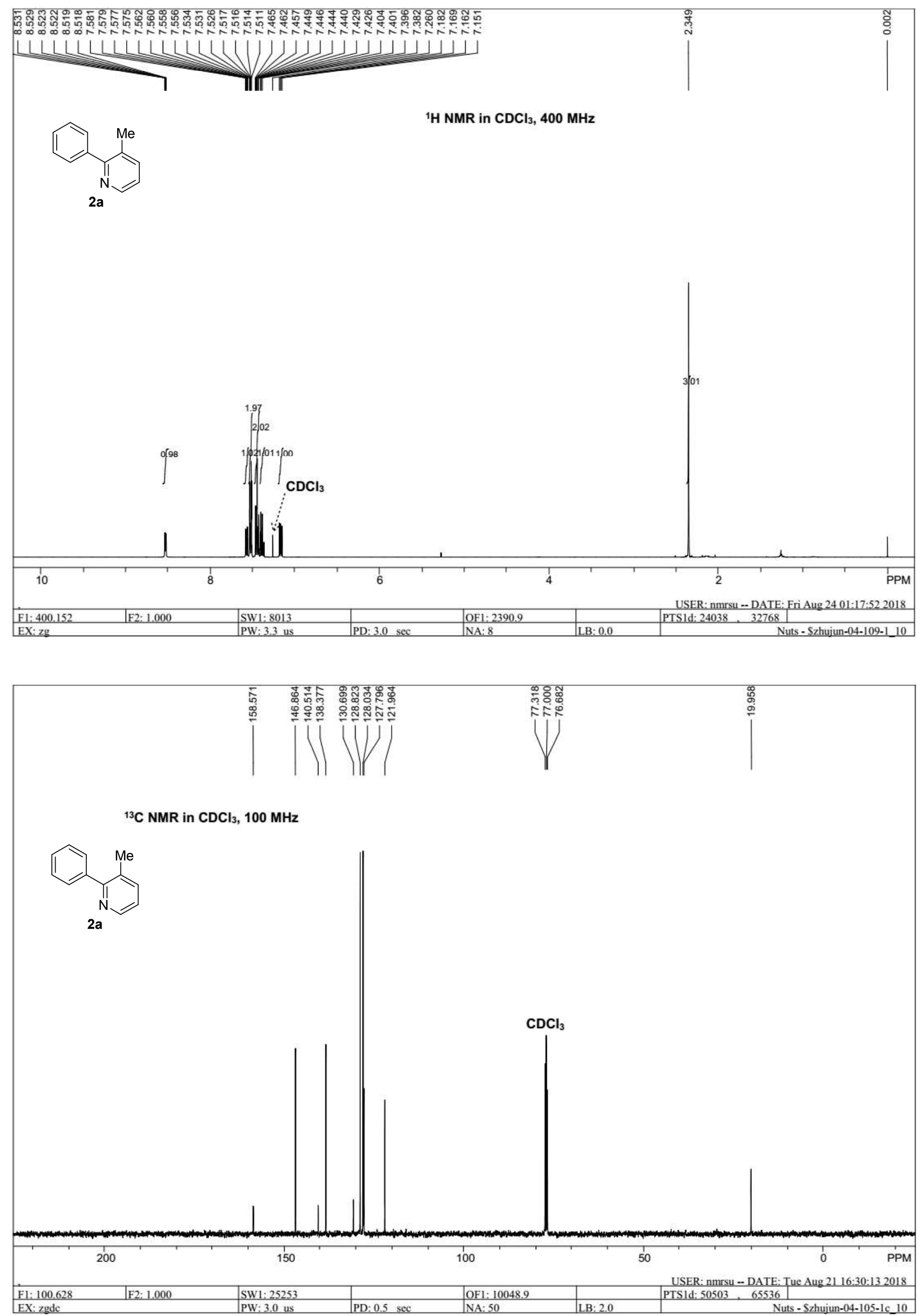

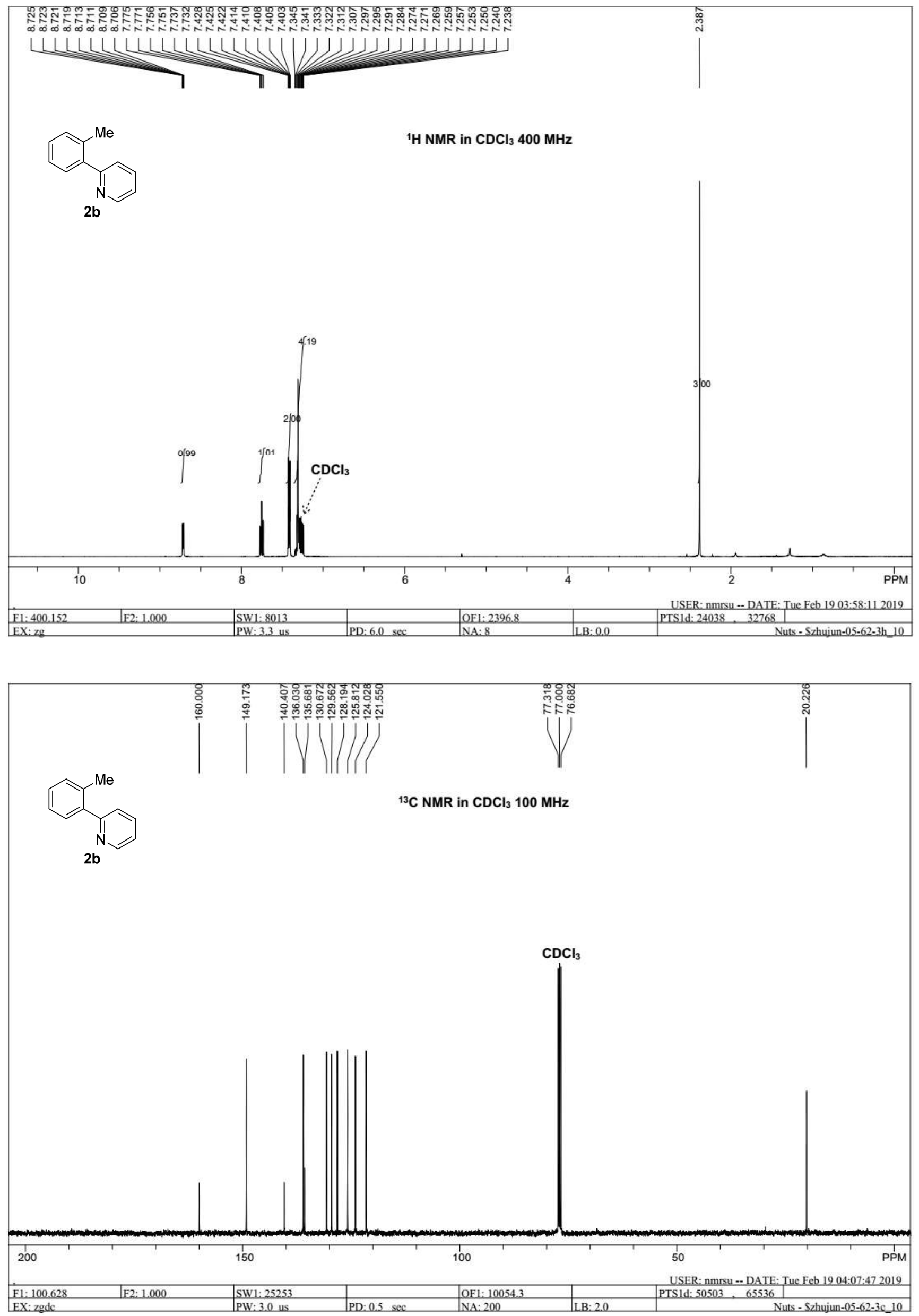

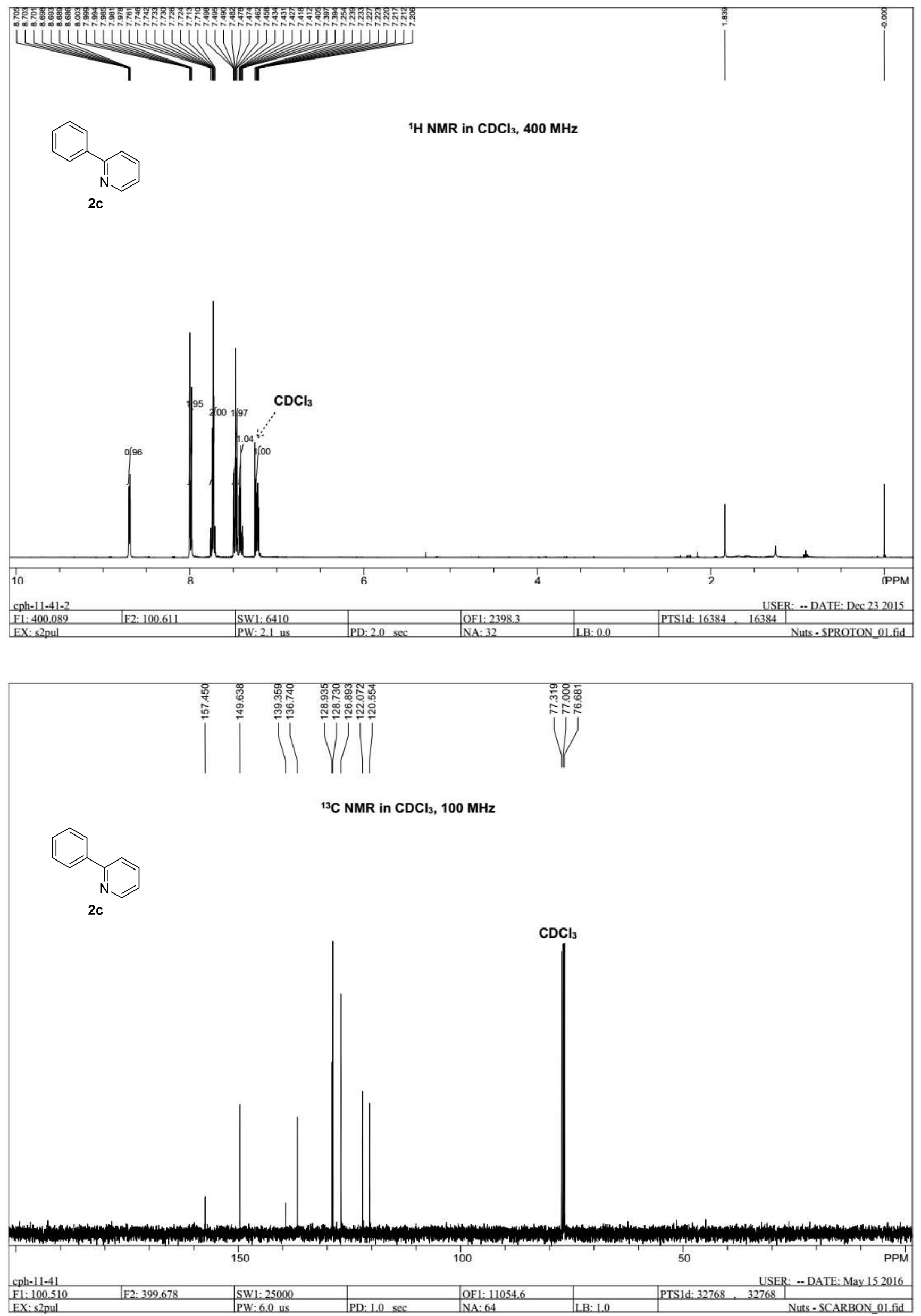

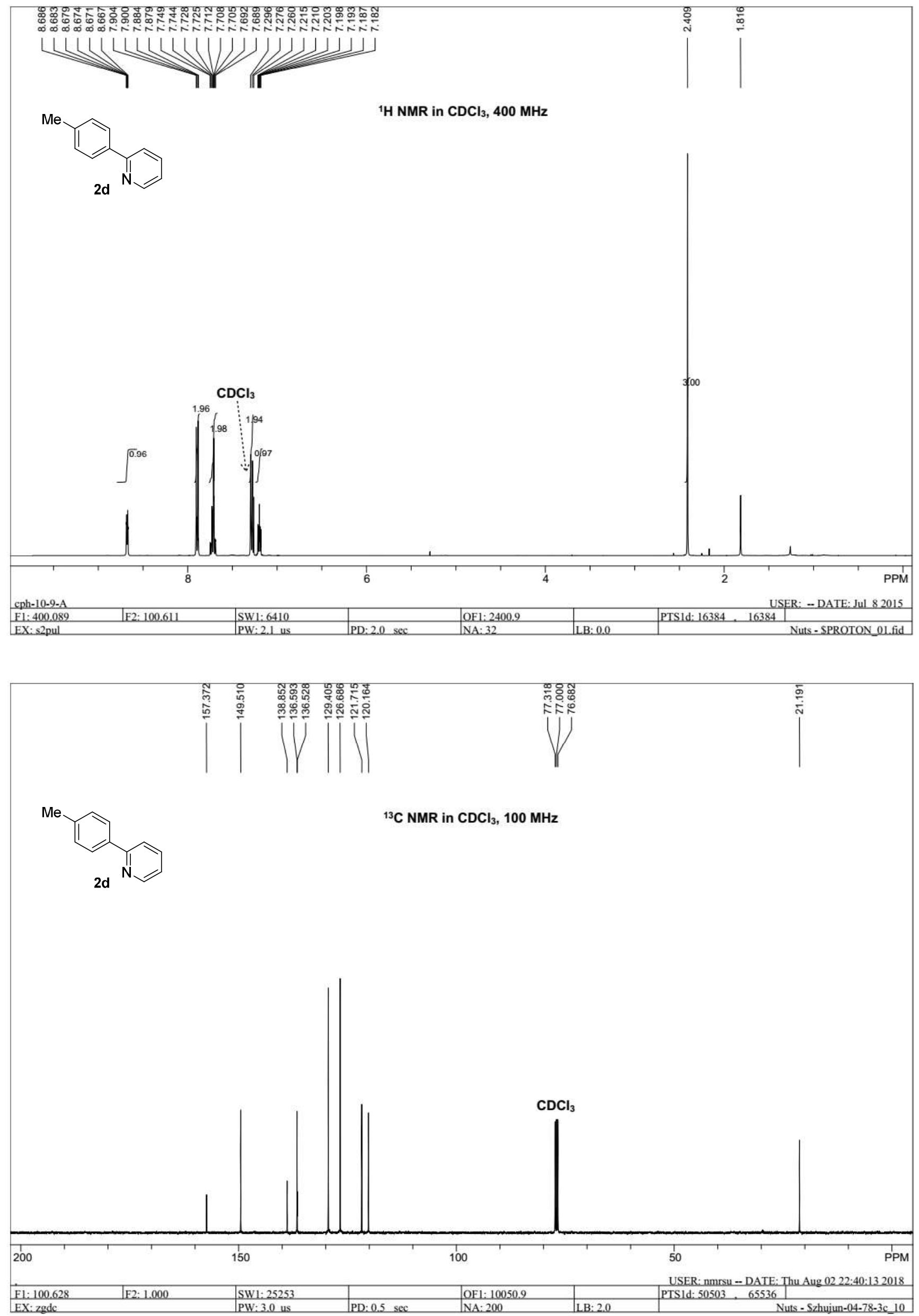

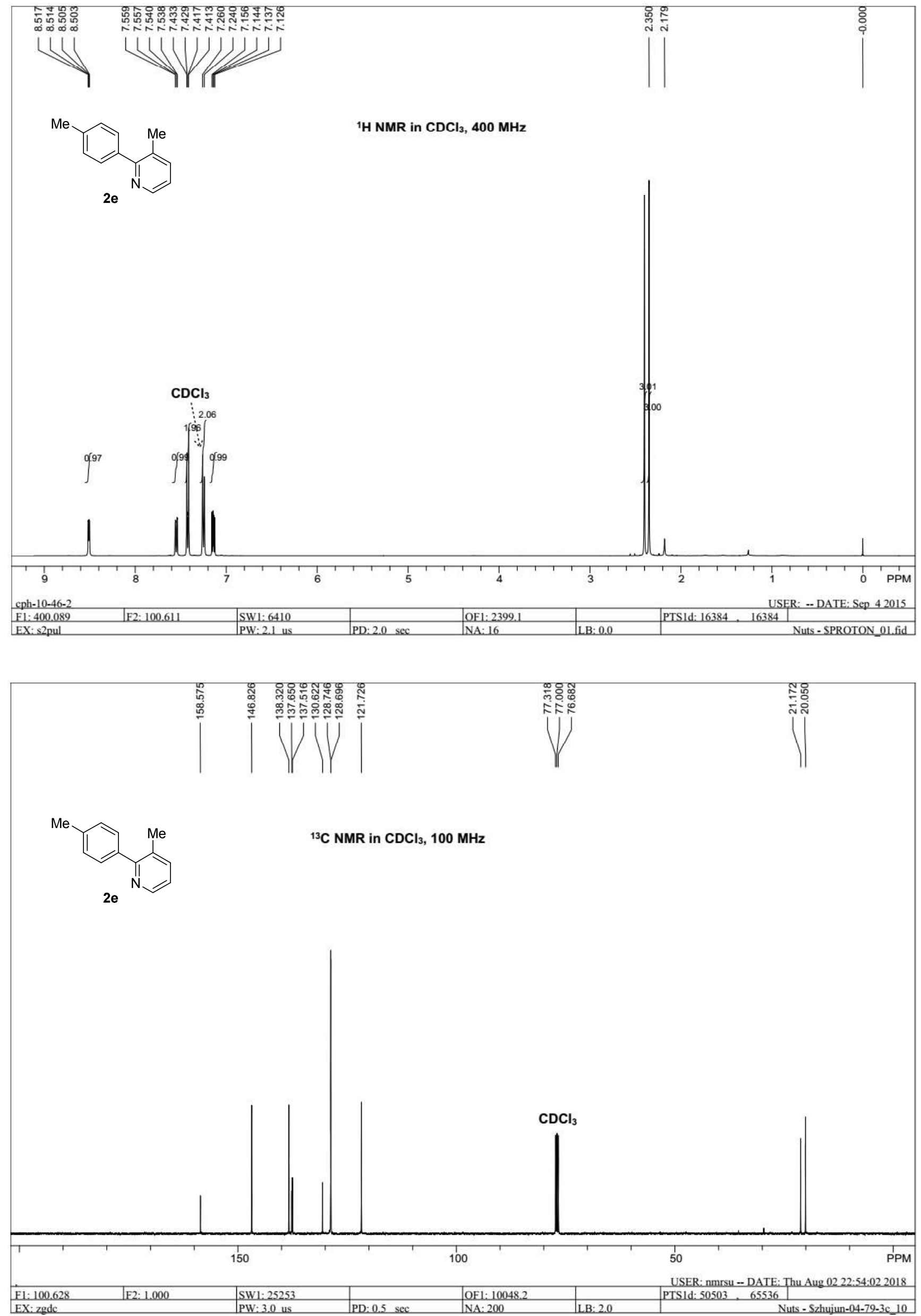

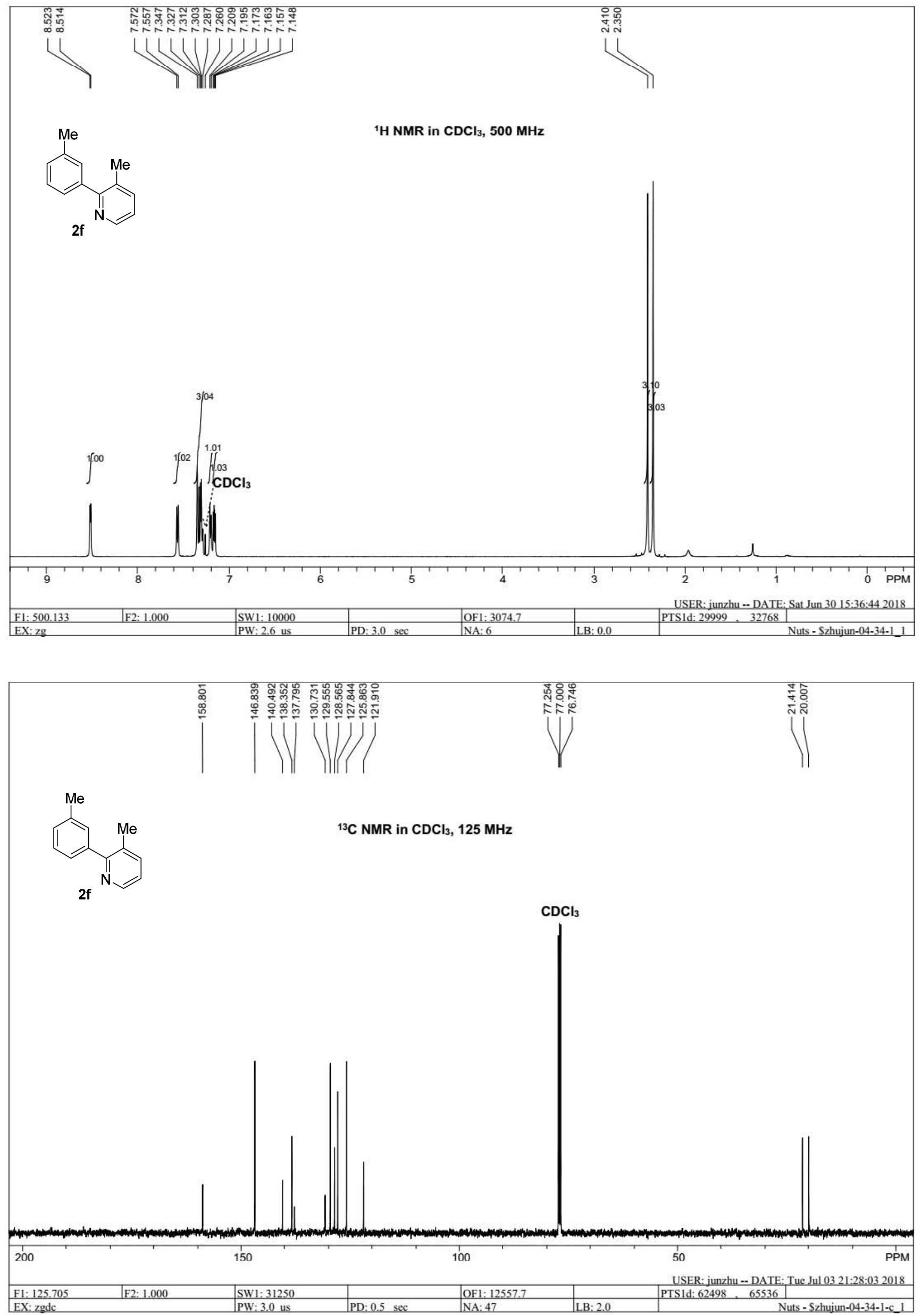

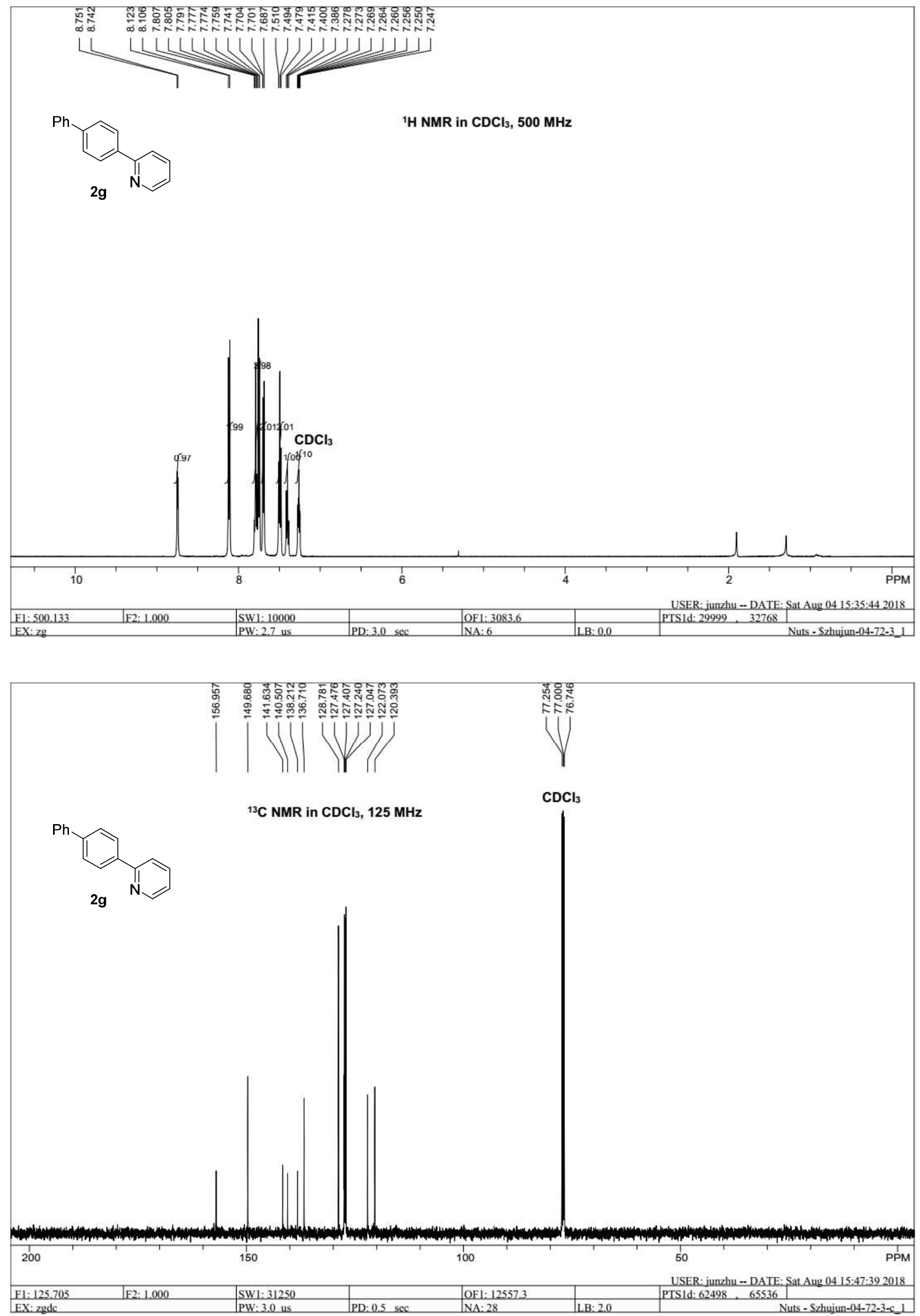

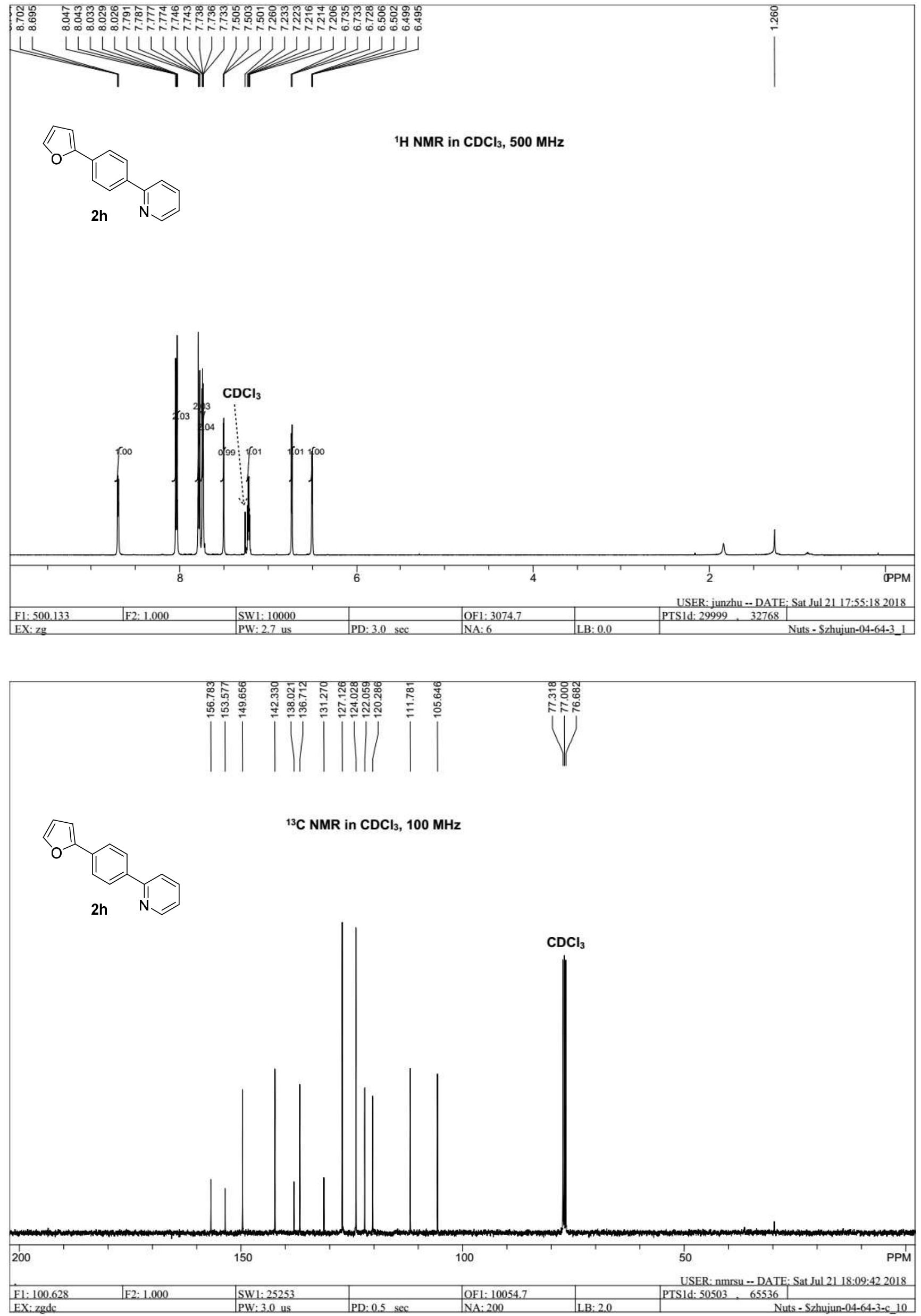

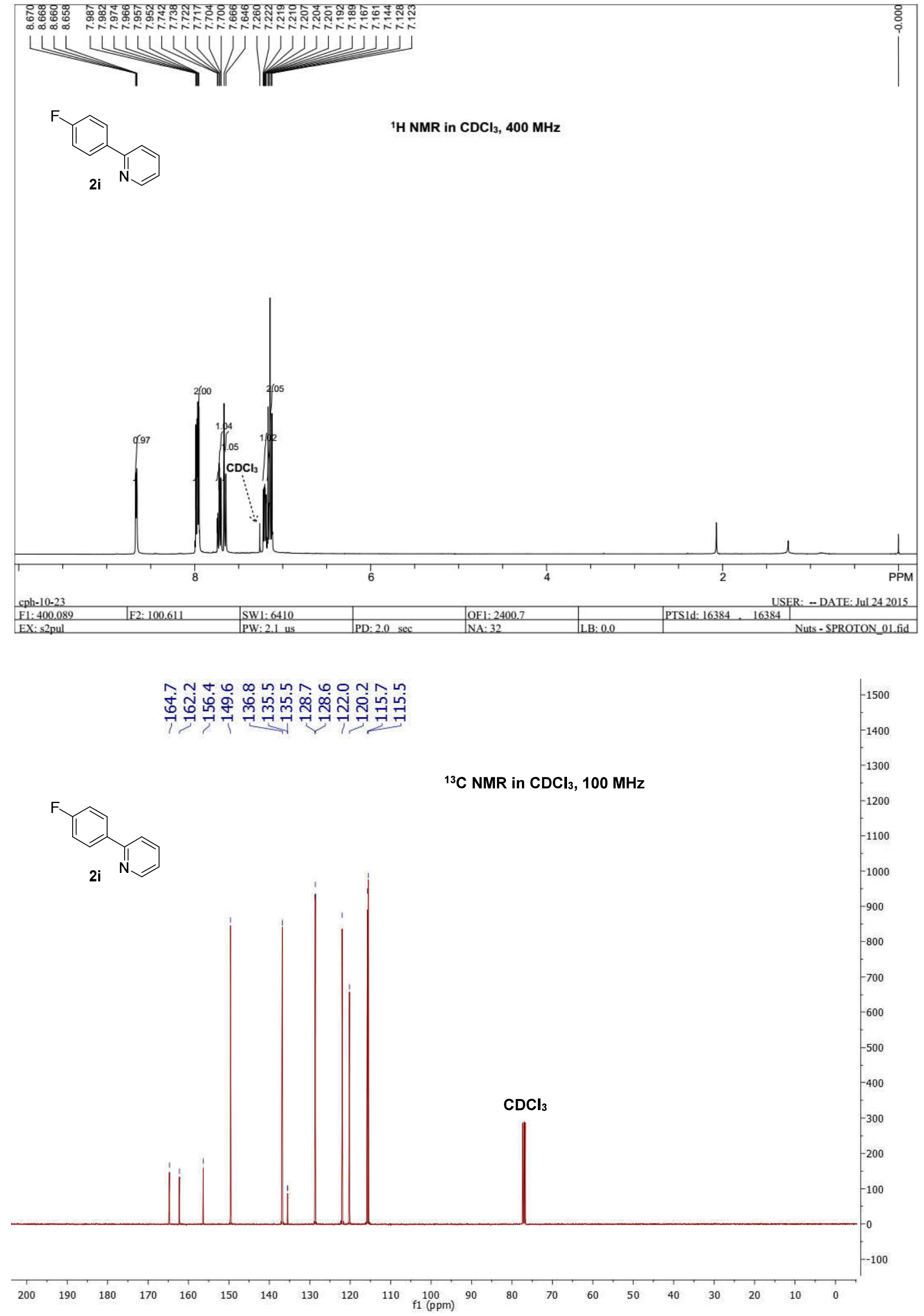

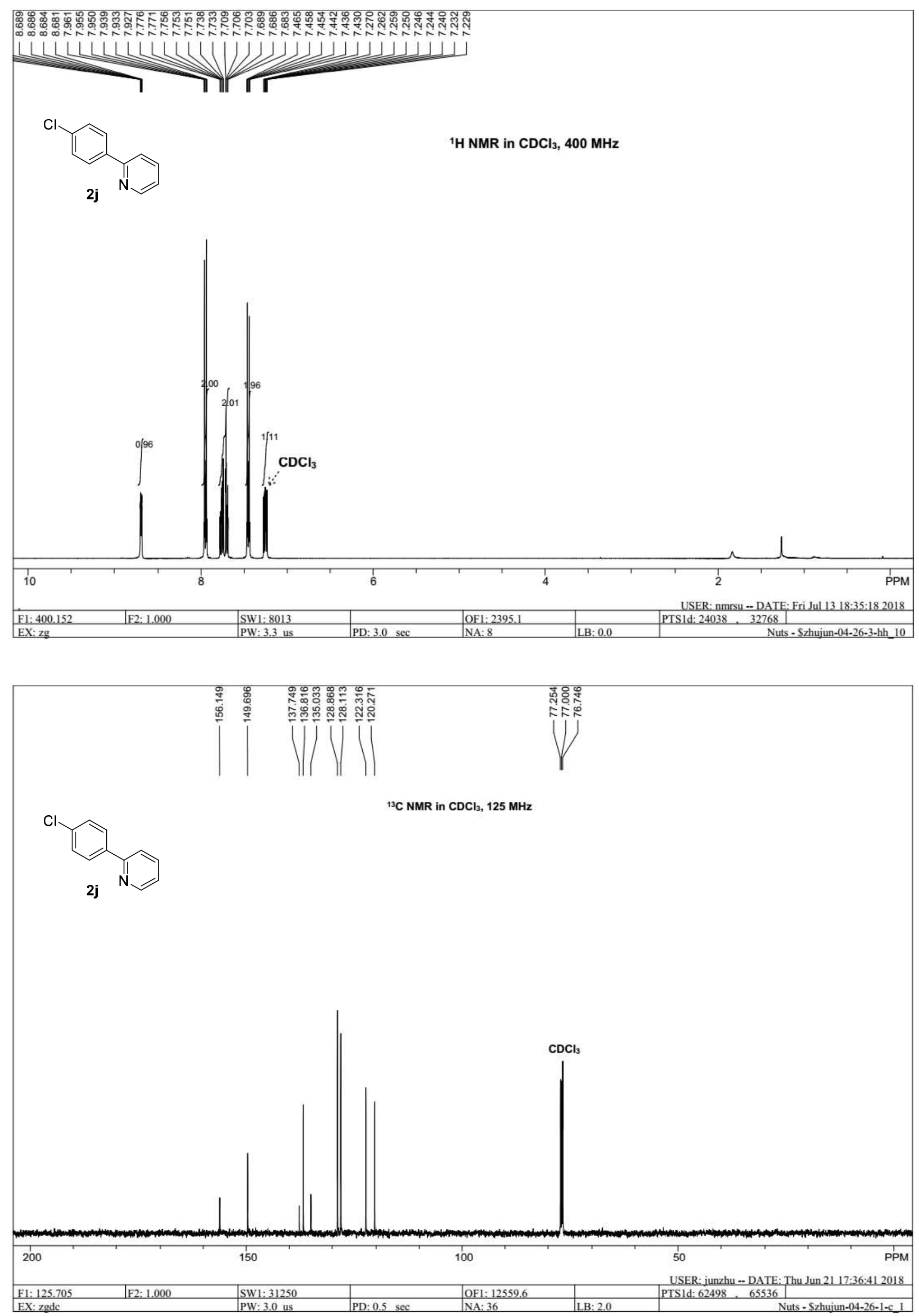

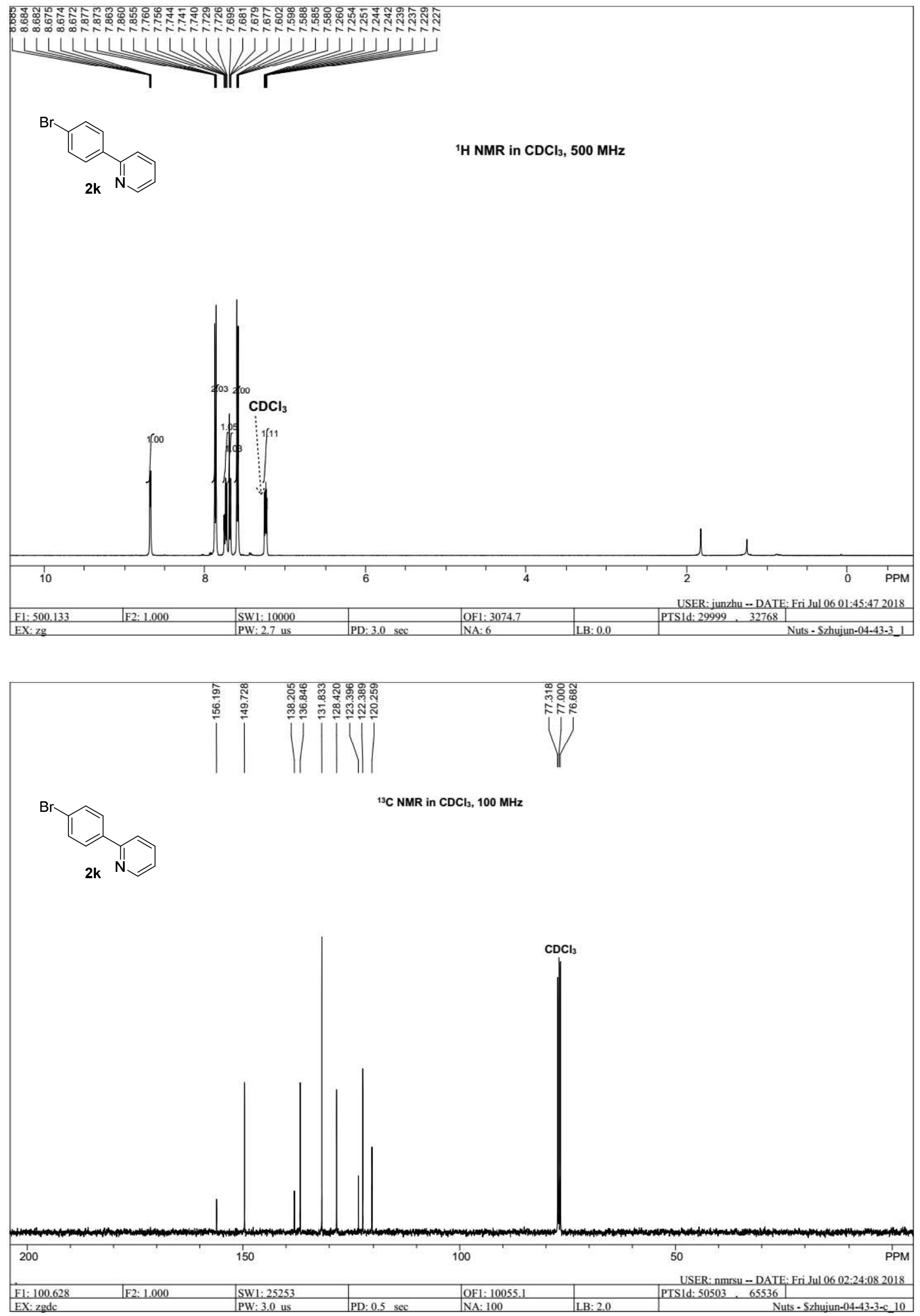

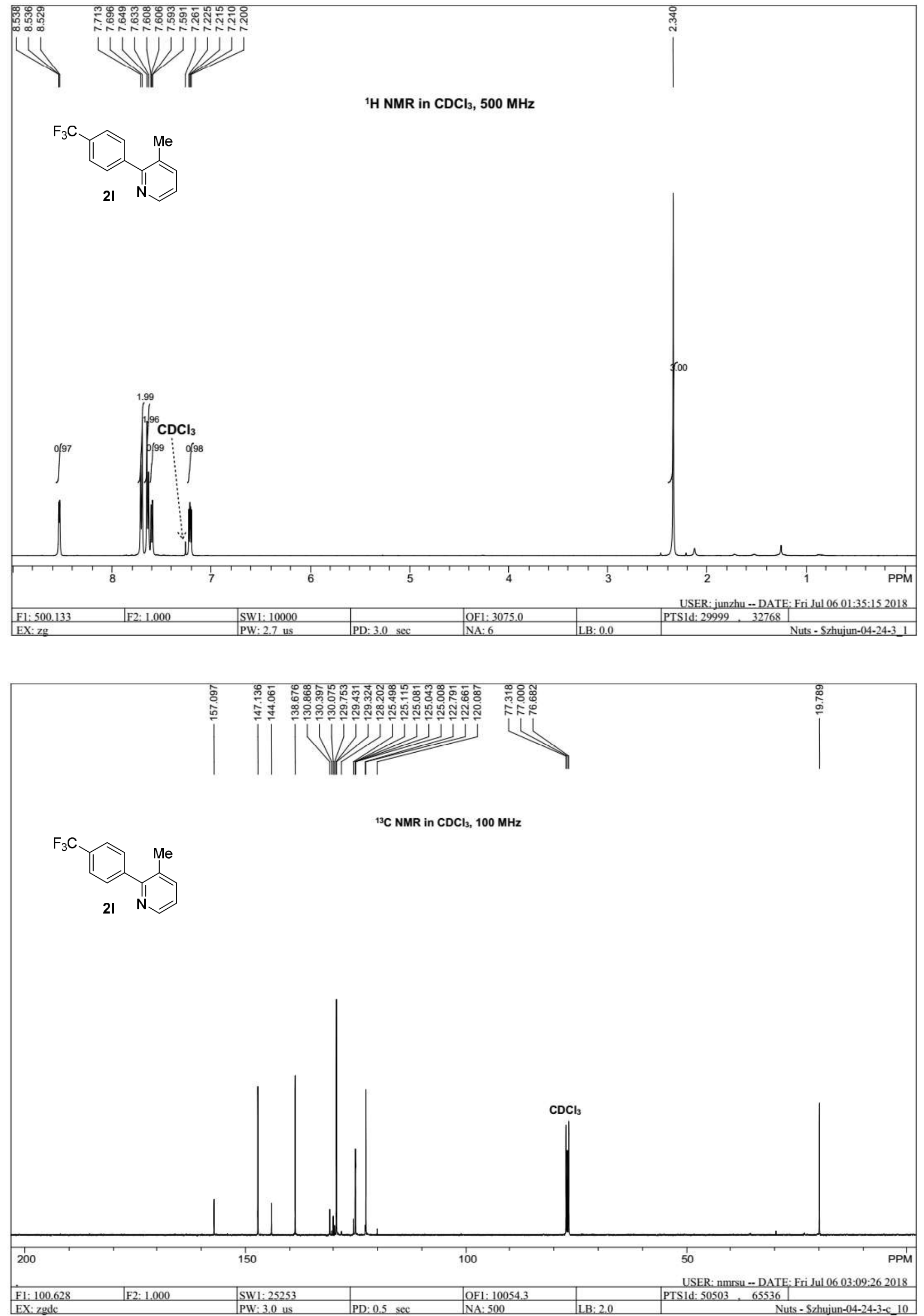


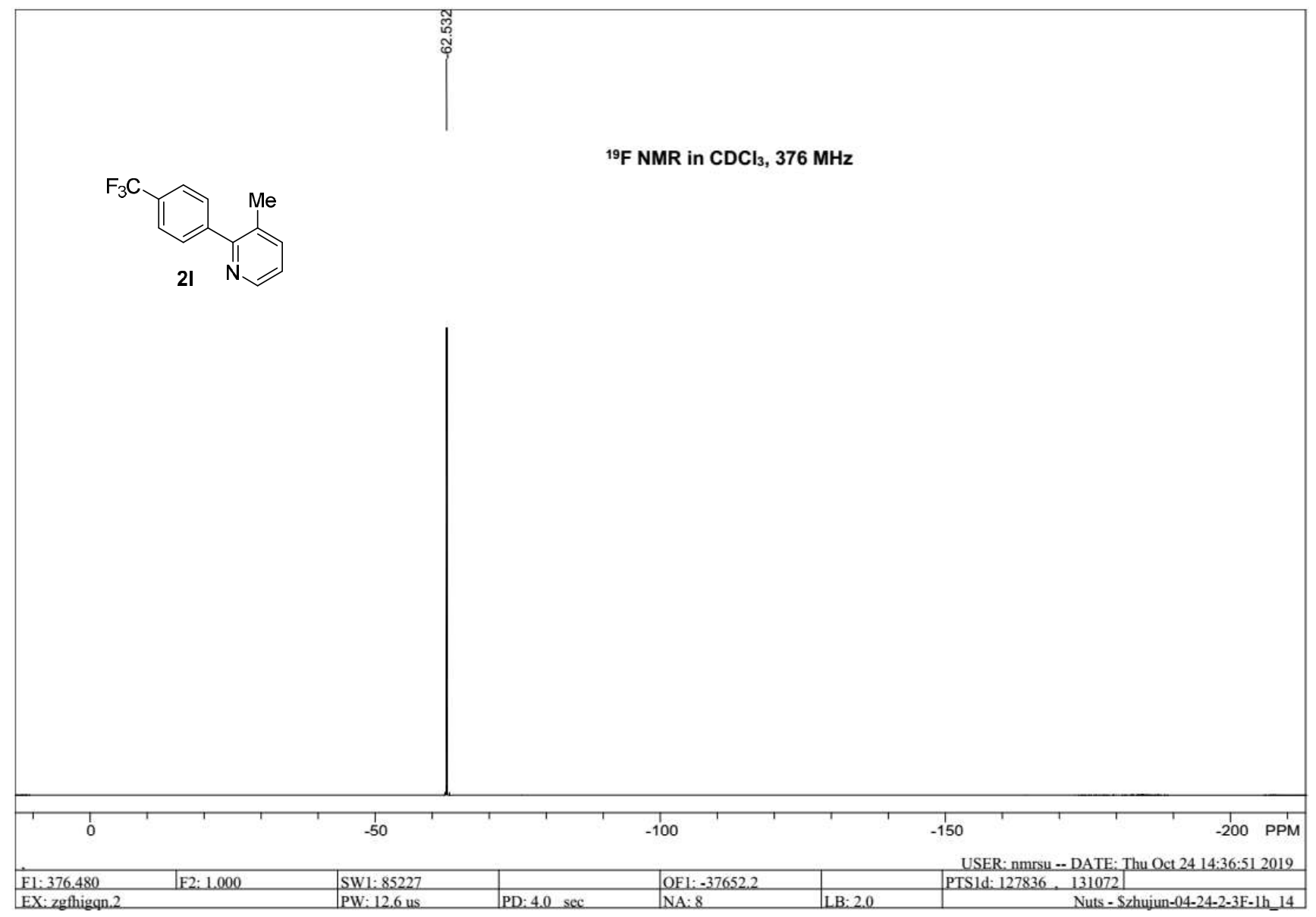



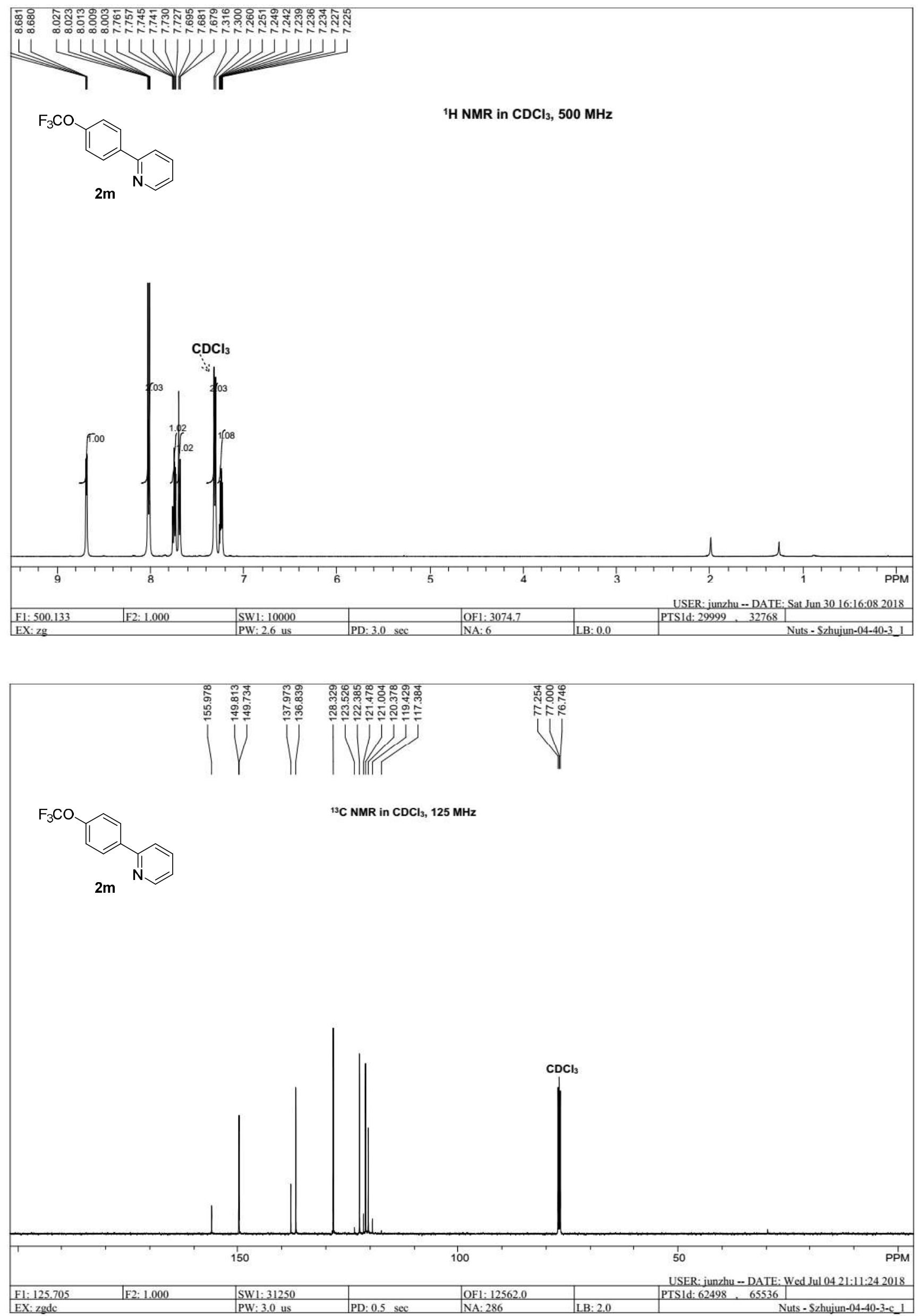


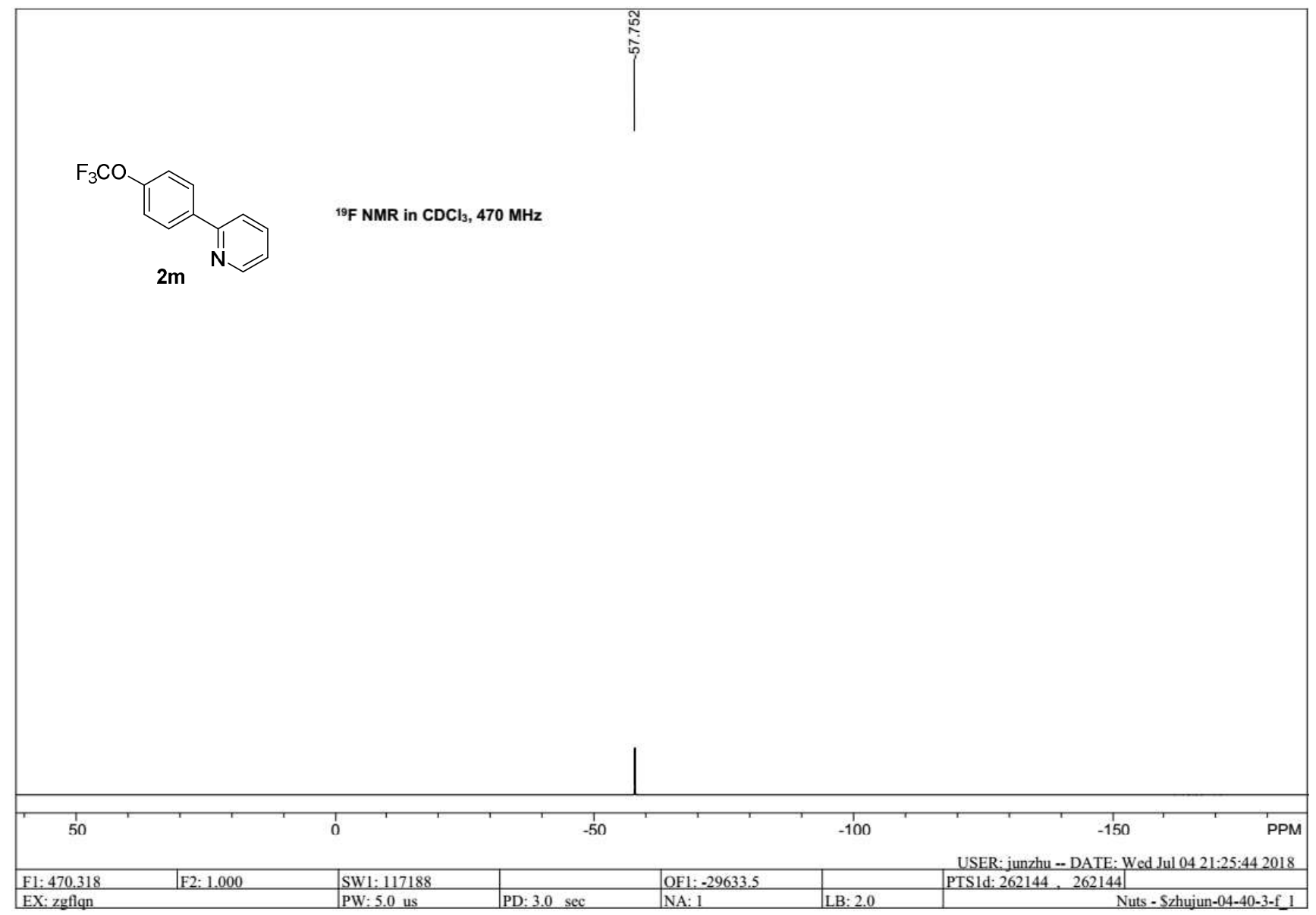



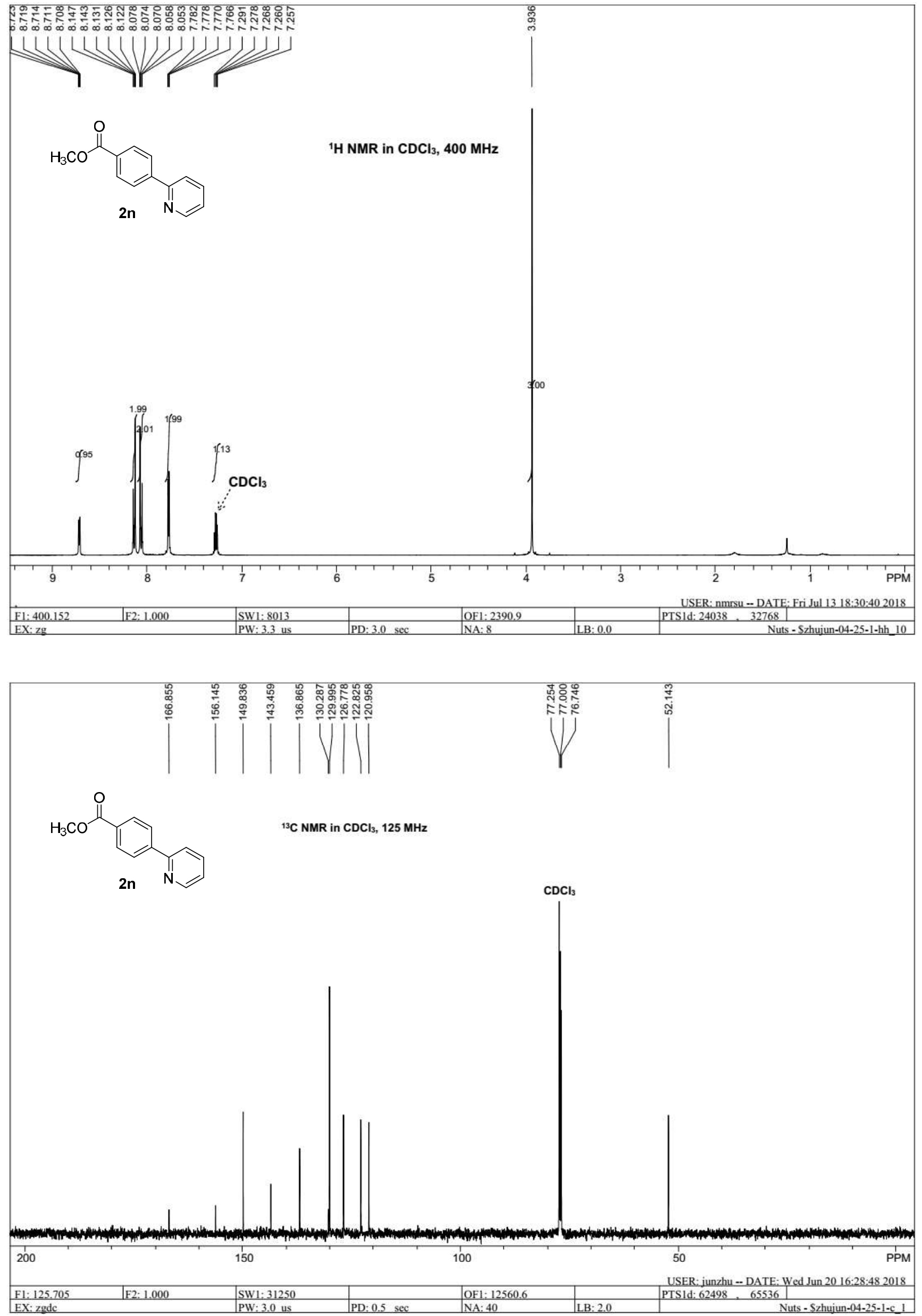

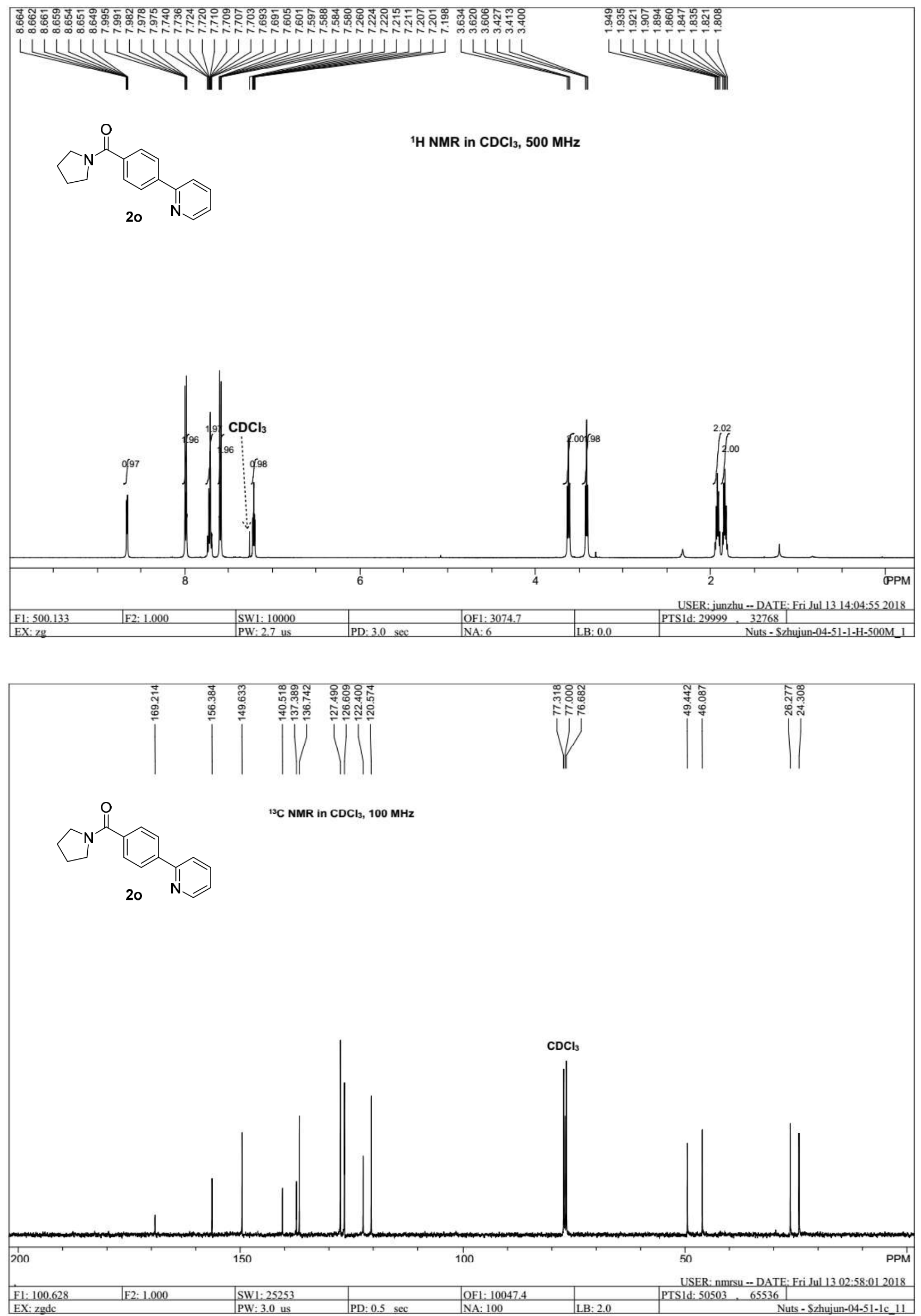

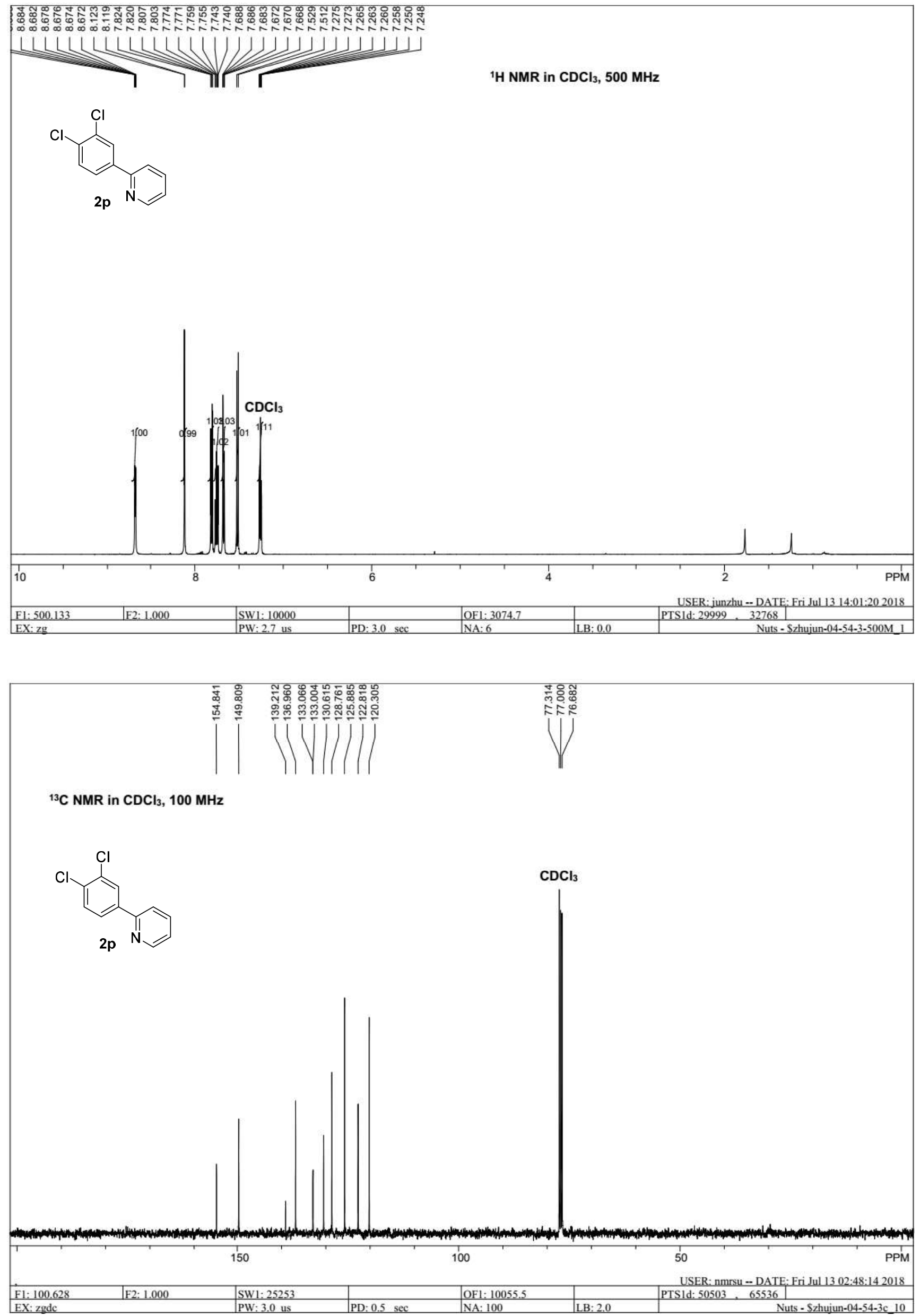

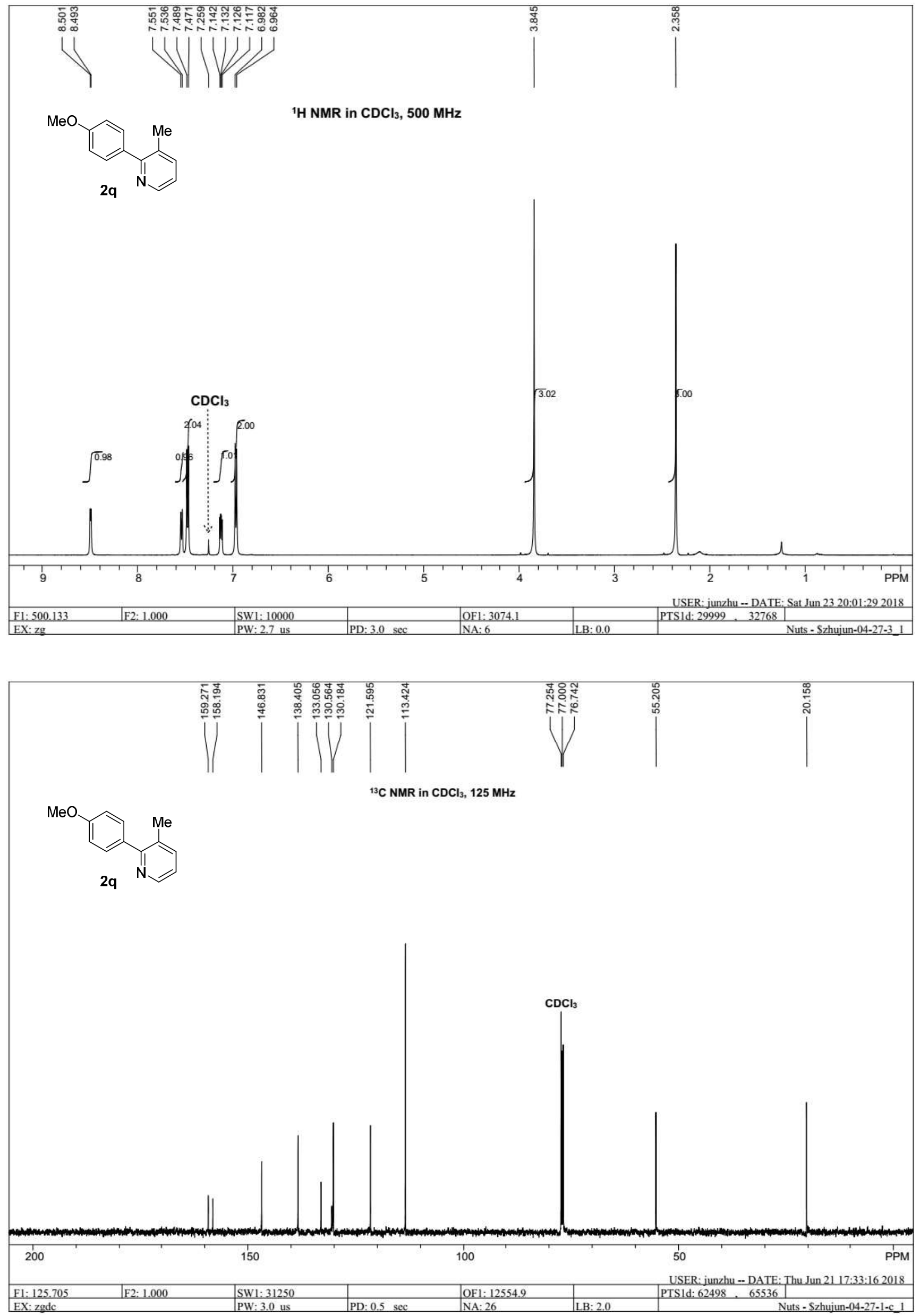

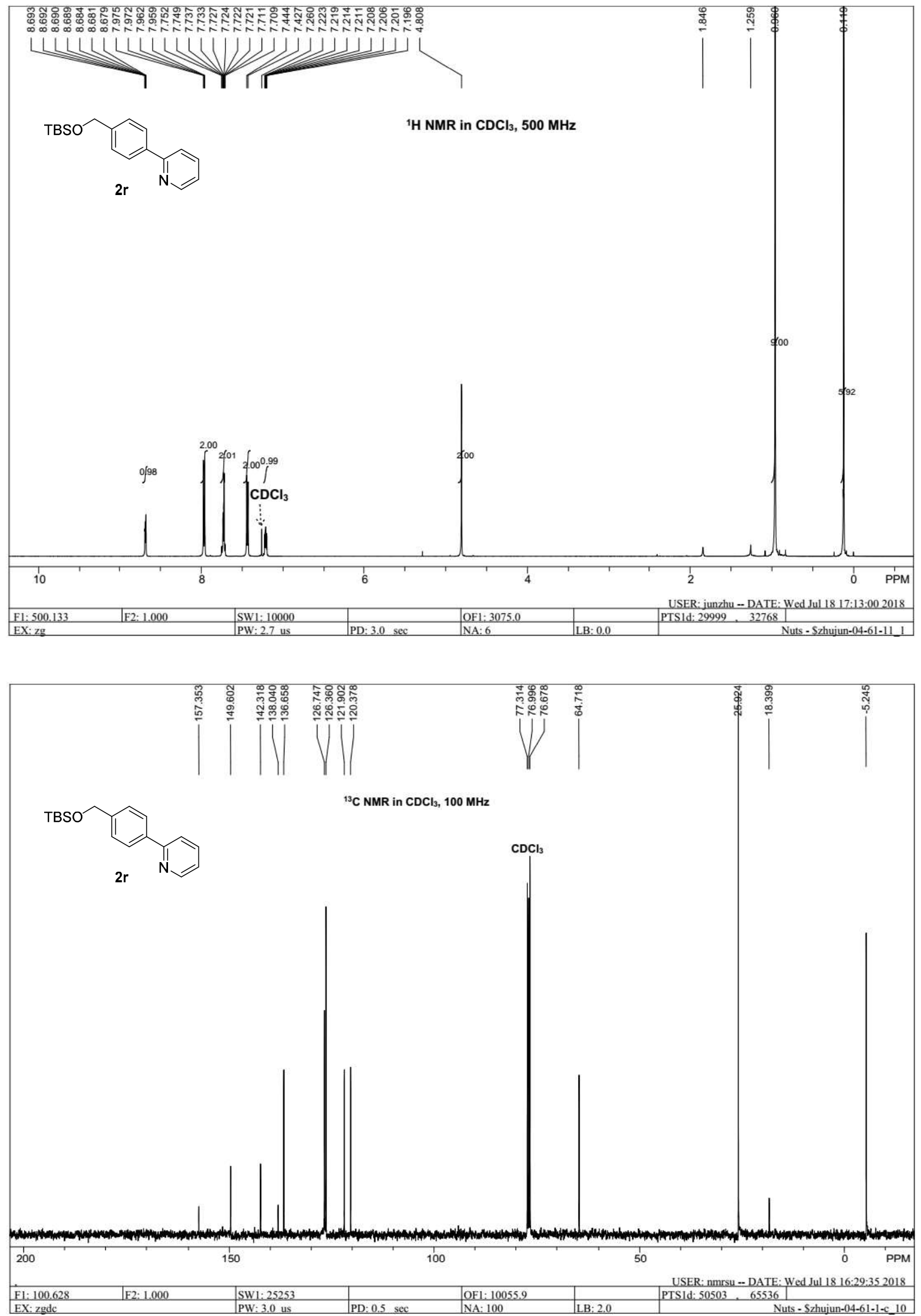

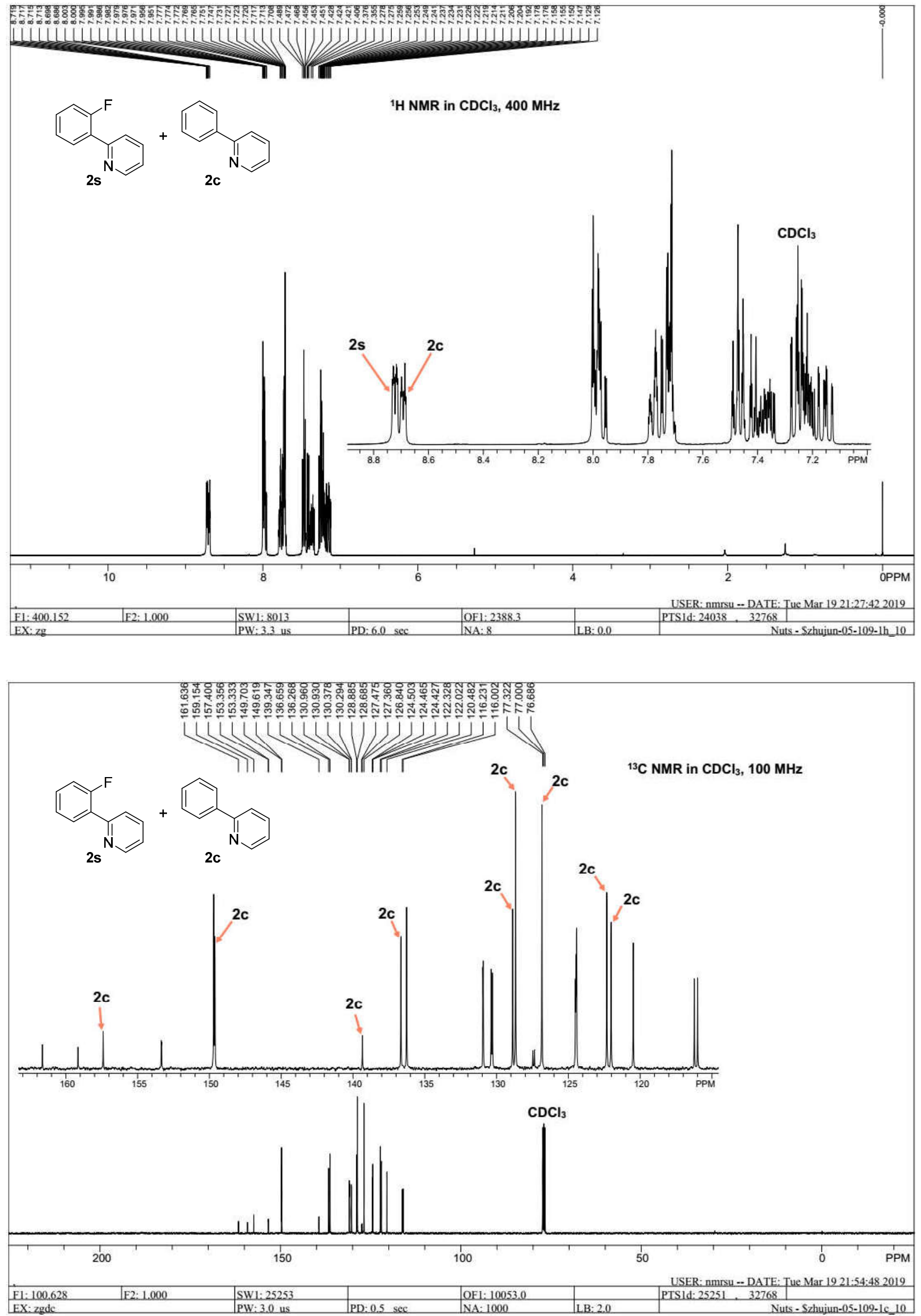


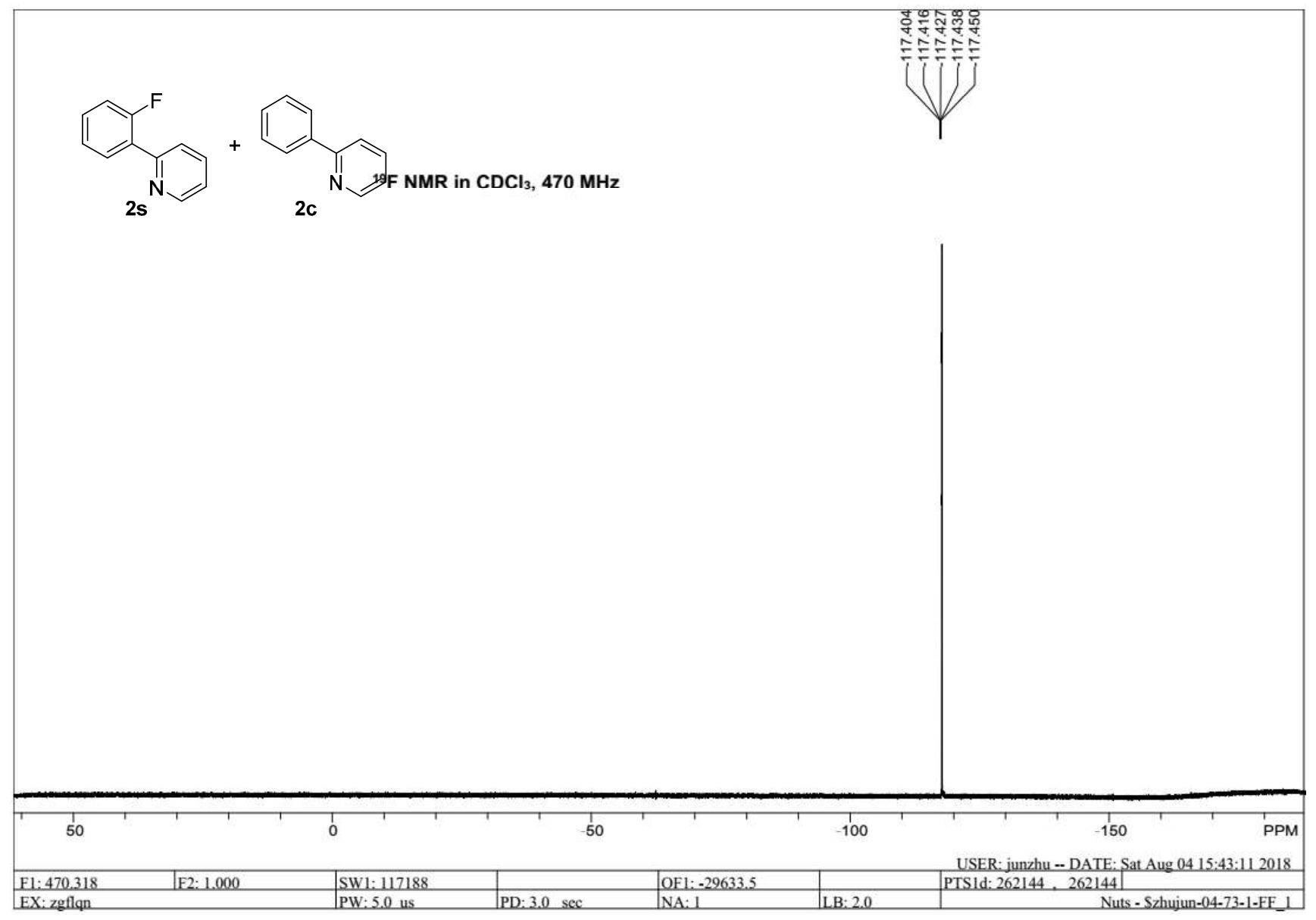



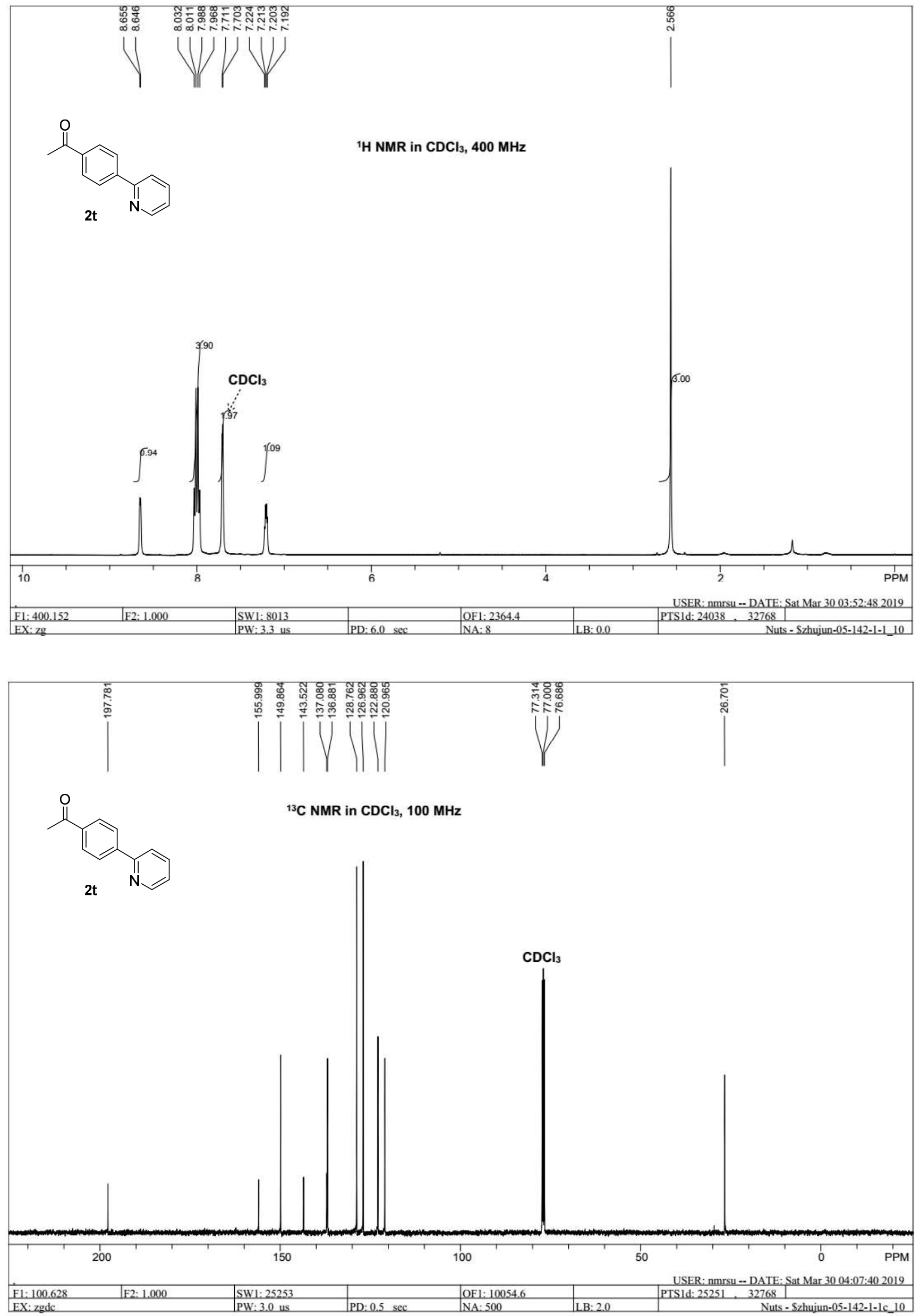

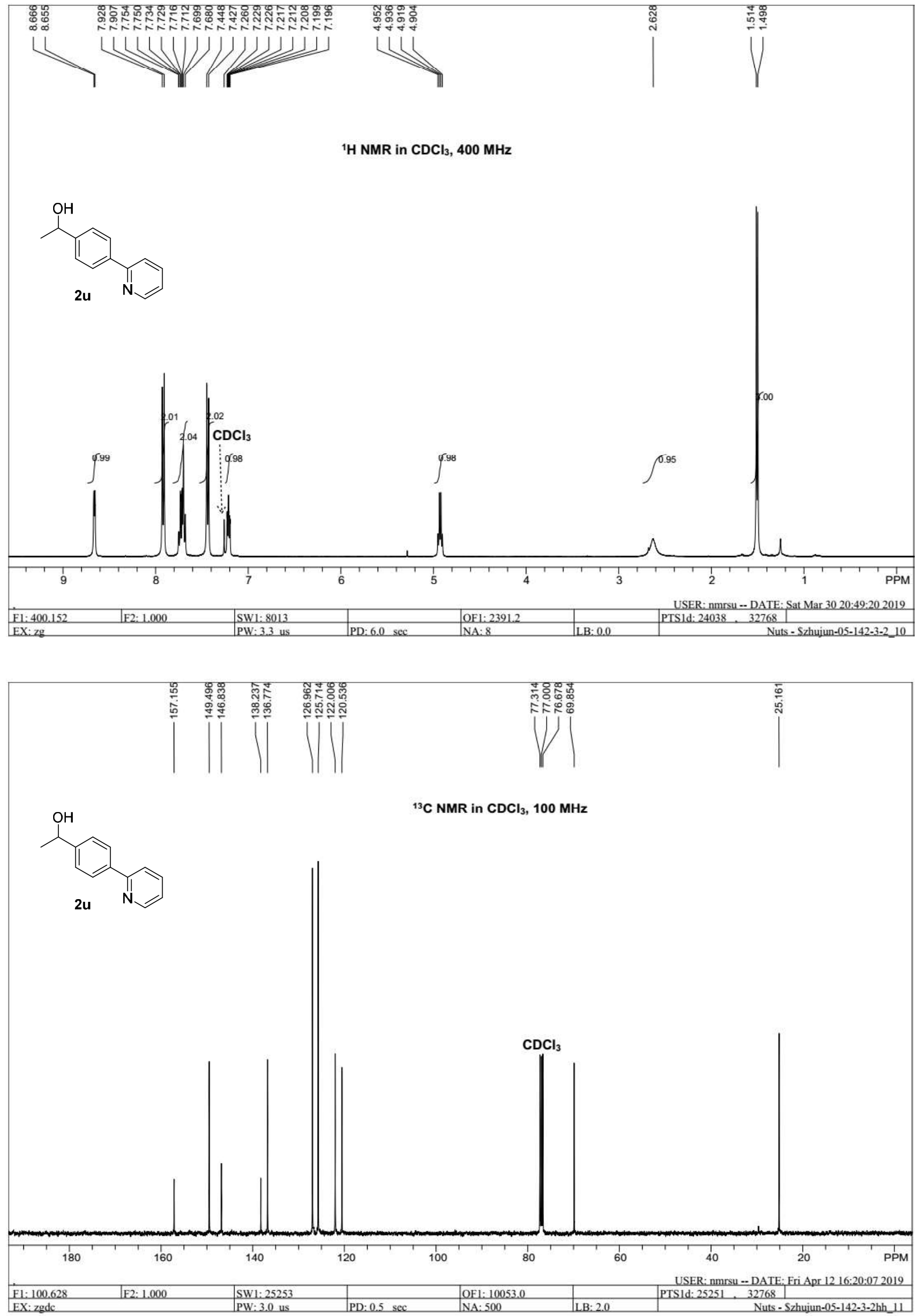

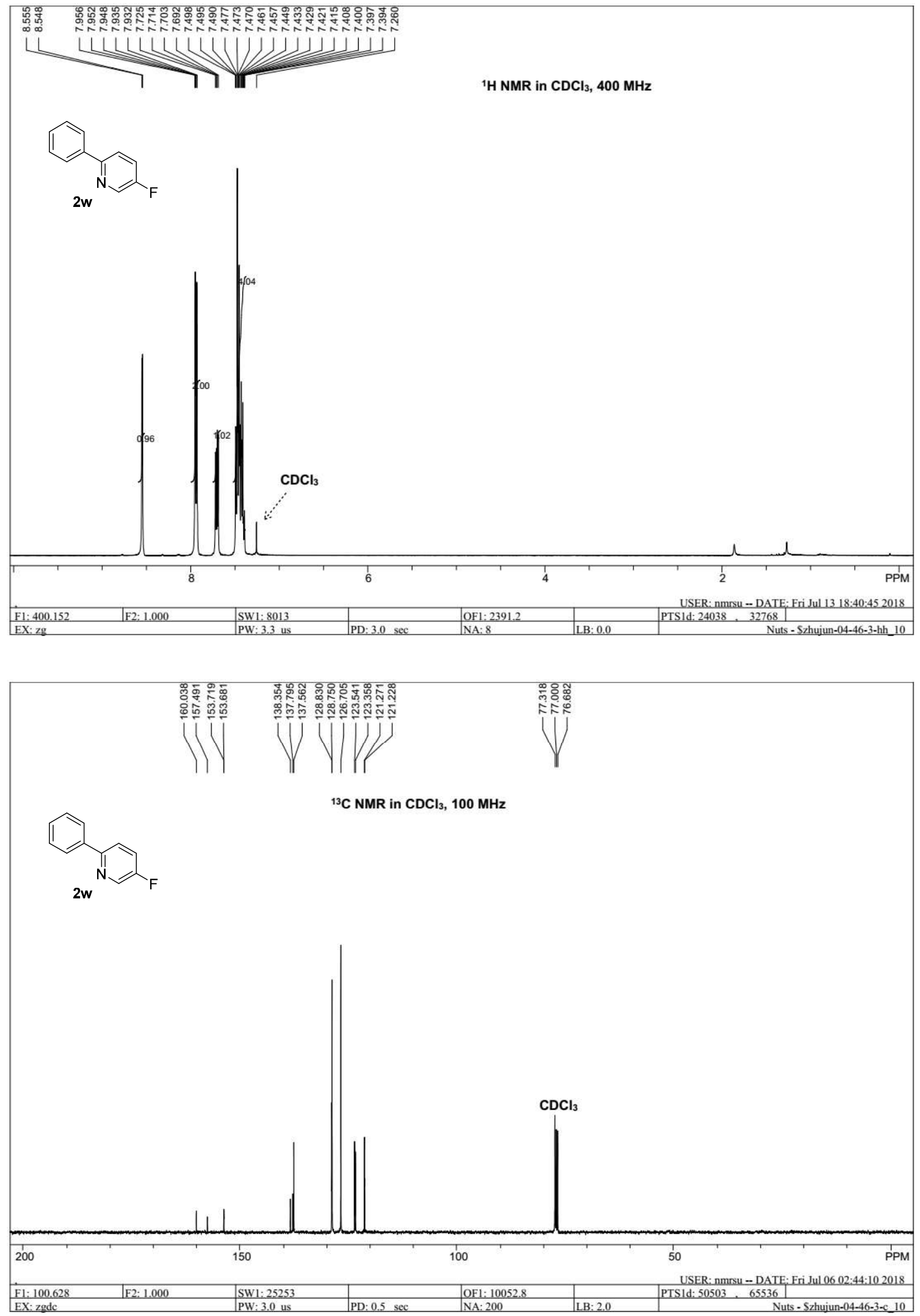


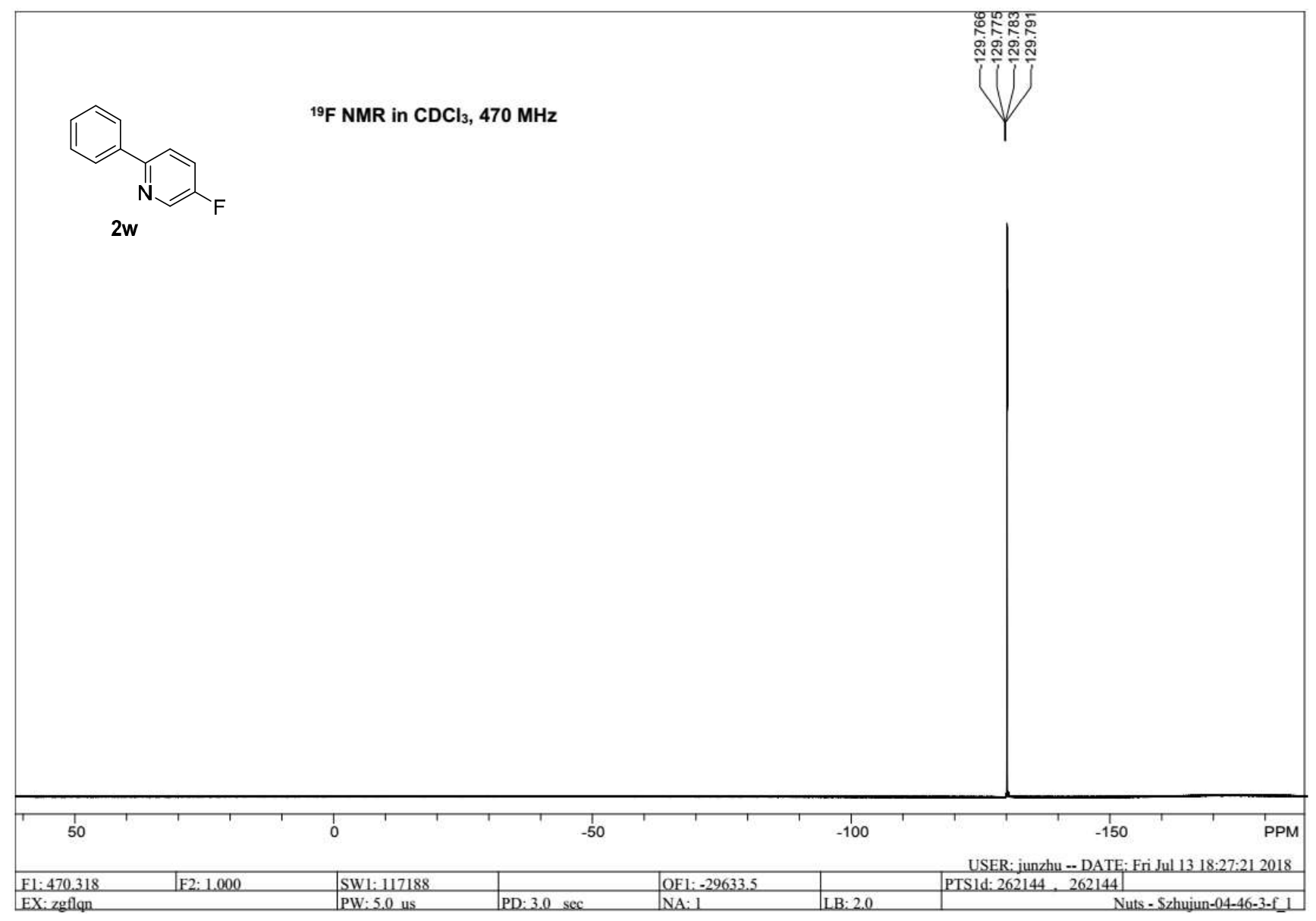



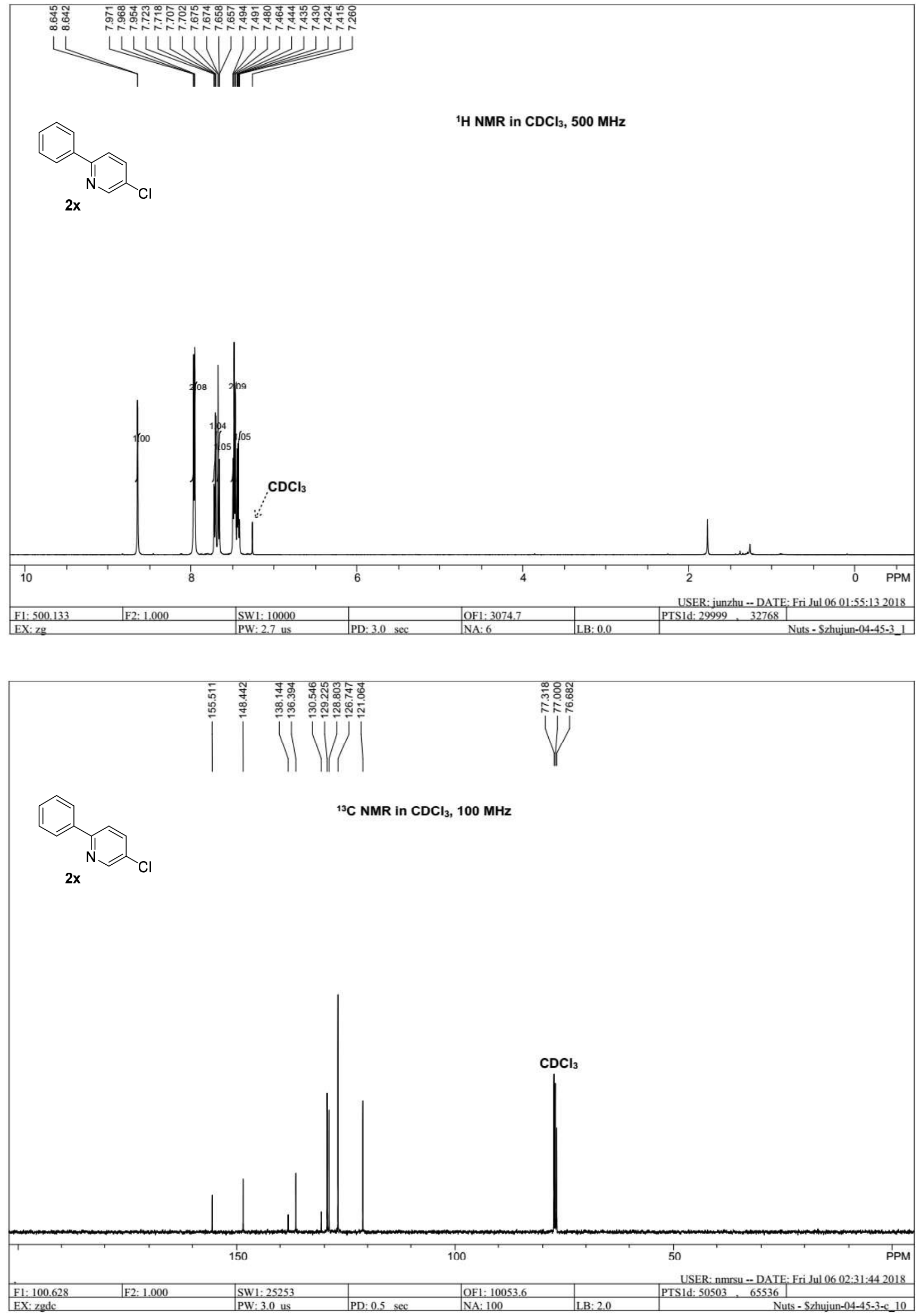

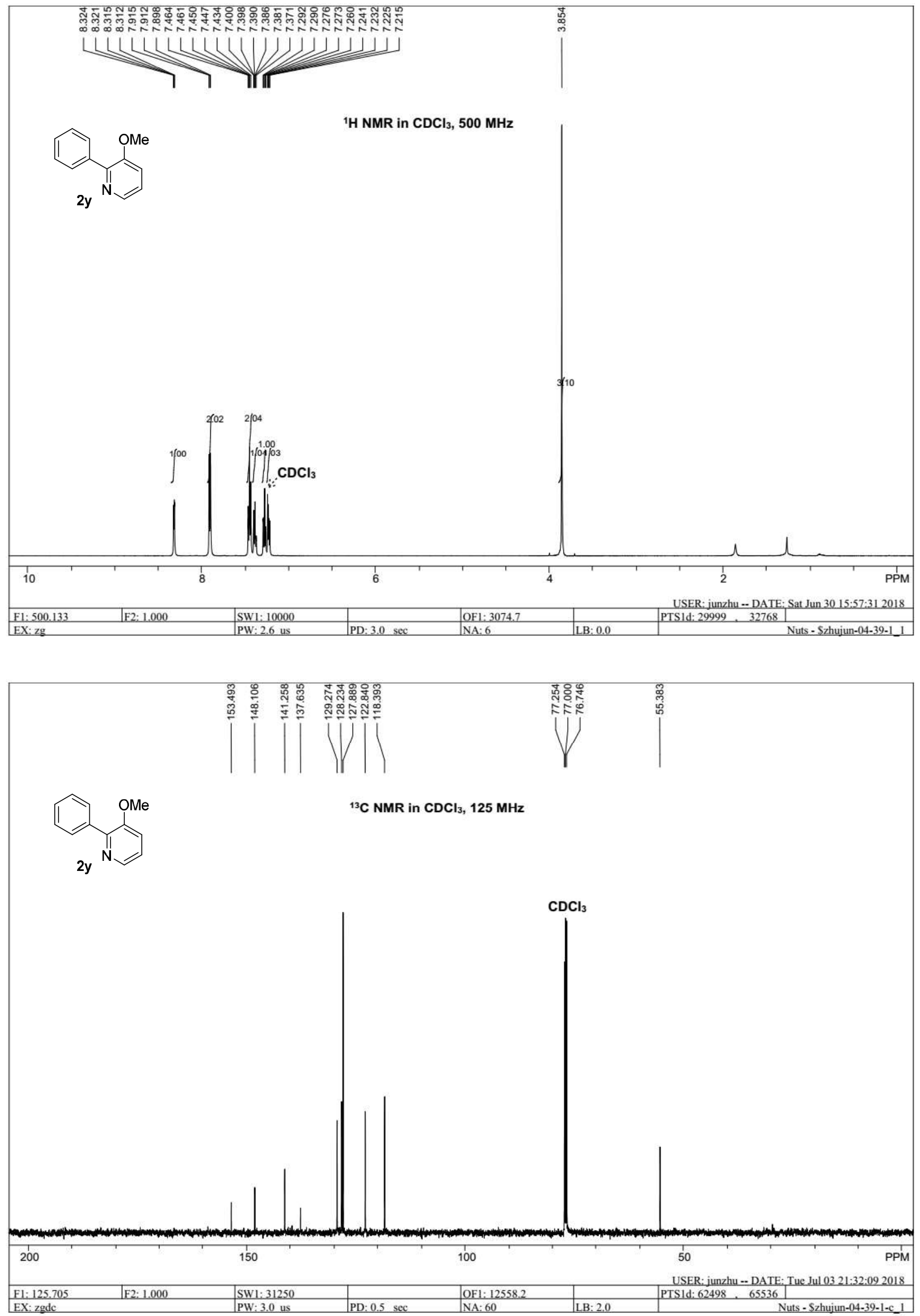

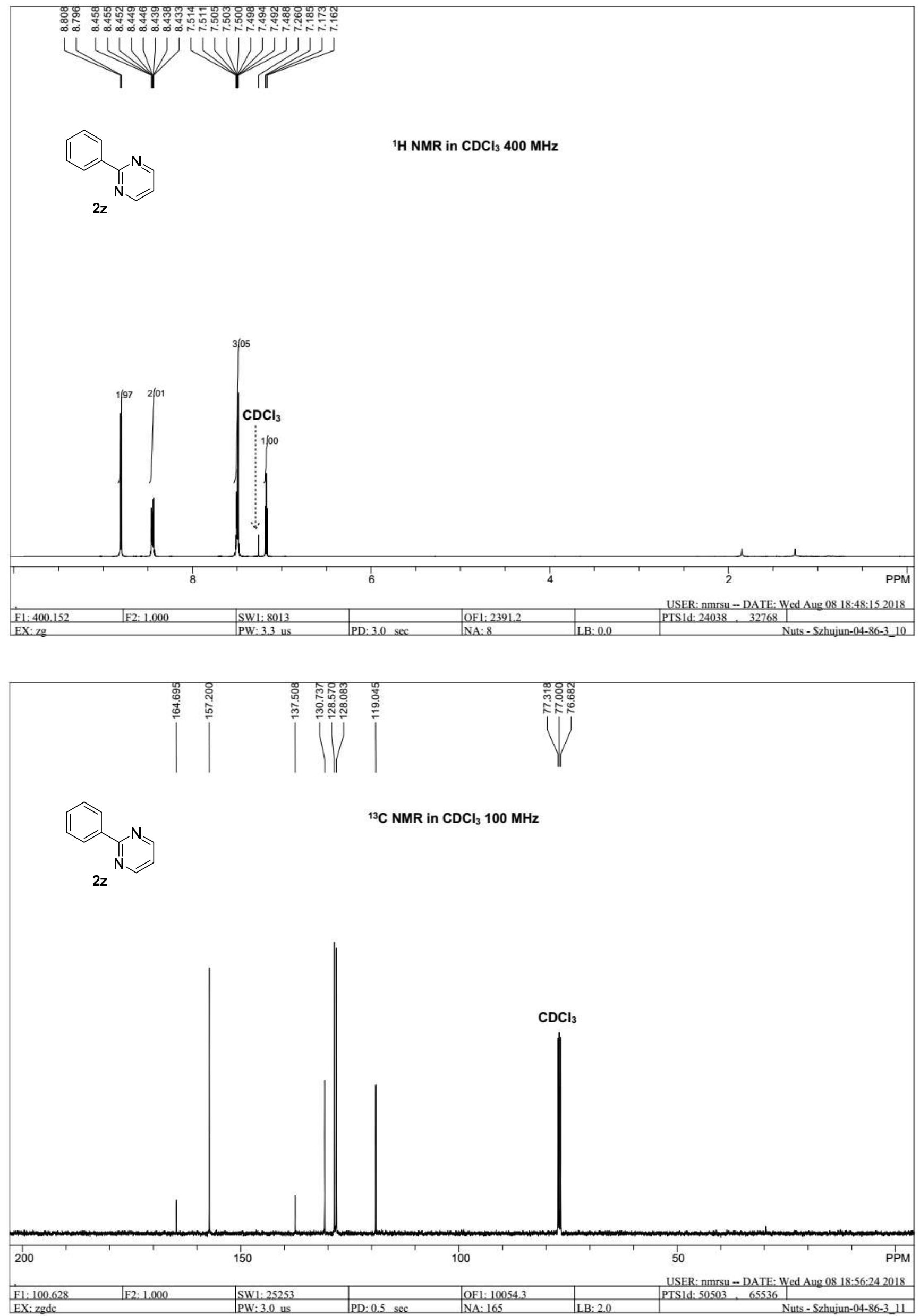

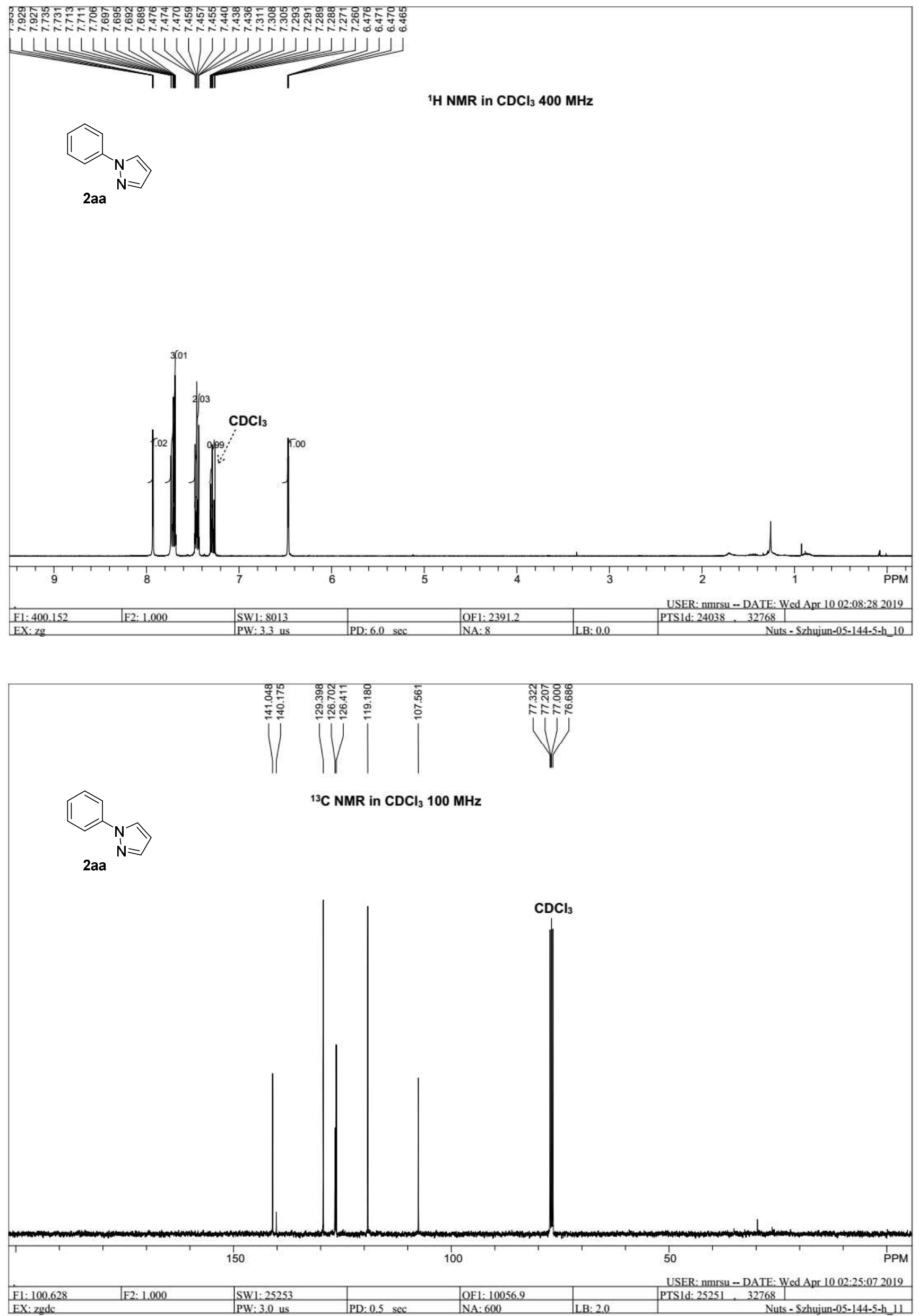

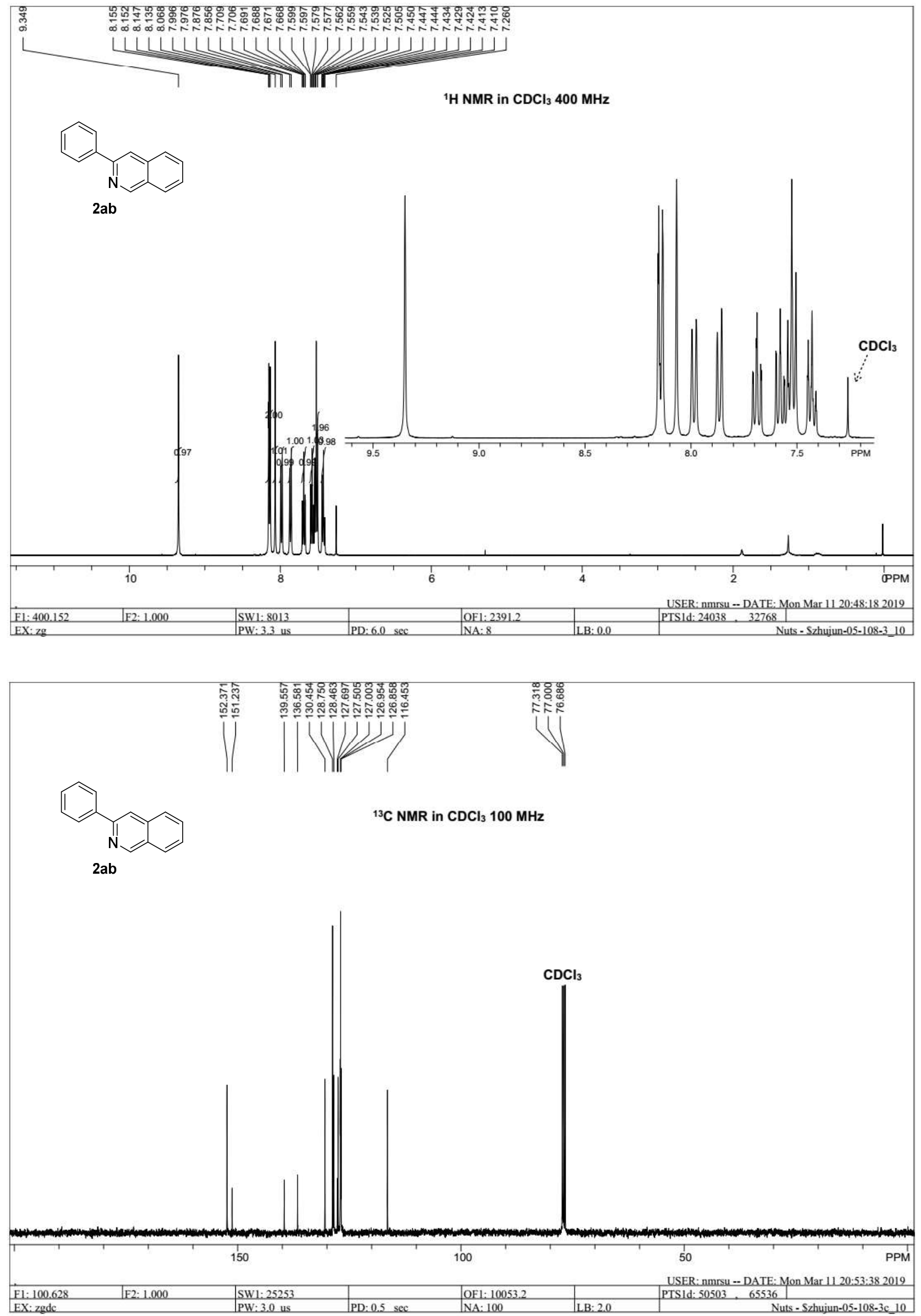

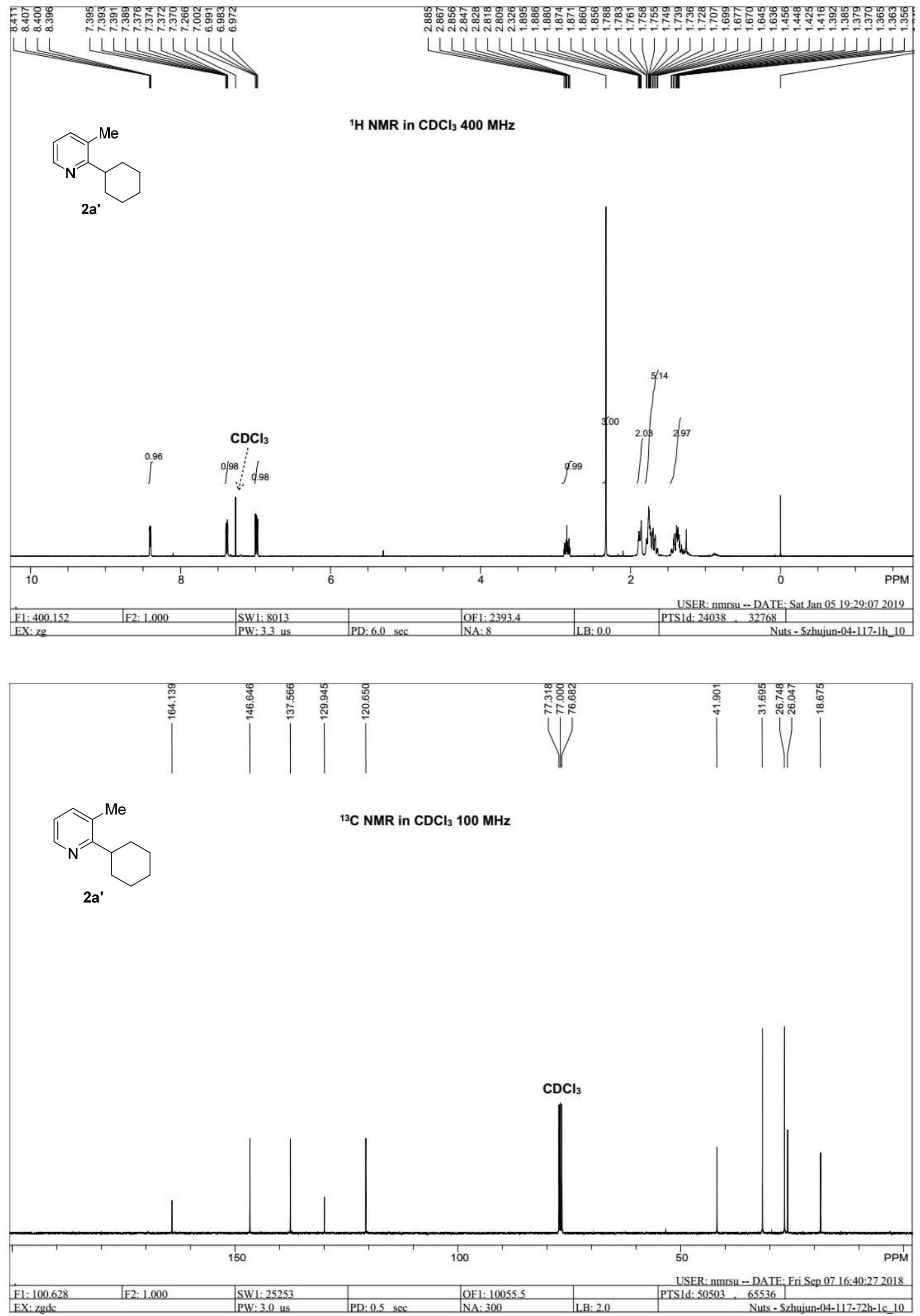